For

\title{
Synthesis of $\alpha$-Fluoroketones from Vinyl Azides and Mechanism Interrogation
}

\author{
Shu-Wei Wu and Feng Liu* \\ Jiangsu Key Laboratory of Translational Research and Therapy for Neuro-Psycho-Diseases and \\ Department of Medicinal Chemistry, College of Pharmaceutical Sciences, Soochow University, 199 \\ Ren-Ai Road, Suzhou, Jiangsu 215123, People's Republic of China \\ E-mail: fliu2@,suda.edu.cn
}

\section{Table of Contents}

1. General remarks $\quad$ S2

2. Synthesis of vinyl azides $\quad$ S2

3. Typical experimental procedure S3

4. The reaction of $\mathbf{1 k} \quad$ S3

5. References for known products $\quad$ S4

6. Isotope labeling experiments S5

$\begin{array}{ll}\text { 7. Characterization of the substrates and products } & \text { S7 }\end{array}$

8. NMR Spectra for the the substrates and products S19 


\section{General remarks}

${ }^{1} \mathrm{H}$ NMR spectra were recorded on 400 or $600 \mathrm{MHz}\left(101\right.$ or $151 \mathrm{MHz}$ for ${ }^{13} \mathrm{C}$ NMR, 376 or $564 \mathrm{MHz}$ for ${ }^{19} \mathrm{~F} \mathrm{NMR}$ ) agilent NMR spectrometer with $\mathrm{CDCl}_{3}$ as the solvent and tetramethylsilane (TMS) as the internal standard. Chemical shifts were reported in parts per million (ppm, $\delta$ scale) downfield from TMS at $0.00 \mathrm{ppm}$ and referenced to the $\mathrm{CDCl}_{3}$ at $7.26 \mathrm{ppm}$ (for ${ }^{1} \mathrm{H} \mathrm{NMR}$ ) or $77.16 \mathrm{ppm}$ (for ${ }^{13} \mathrm{C} \mathrm{NMR}$ ). HRMS was recorded on a GCT Premier ${ }^{\mathrm{TM}}$ (CI) Mass Spectrometer. Infrared (FT-IR) spectra were recorded on a Varian 1000FT-IR, $v_{\max }$ in $\mathrm{cm}^{-1}$. Melting points were measured using SGW, X-4B and values are uncorrected. All commercially available reagents and solvents were used as received unless otherwise specified. The subsrates were readily prepared from phenylethylenes or alkynes (Angew. Chem., Int. Ed. 2014.53, 4390; Org. Lett. 2014, 16, 3668).

\section{Synthesis of vinyl azides}

\section{Synthetic Scheme:}
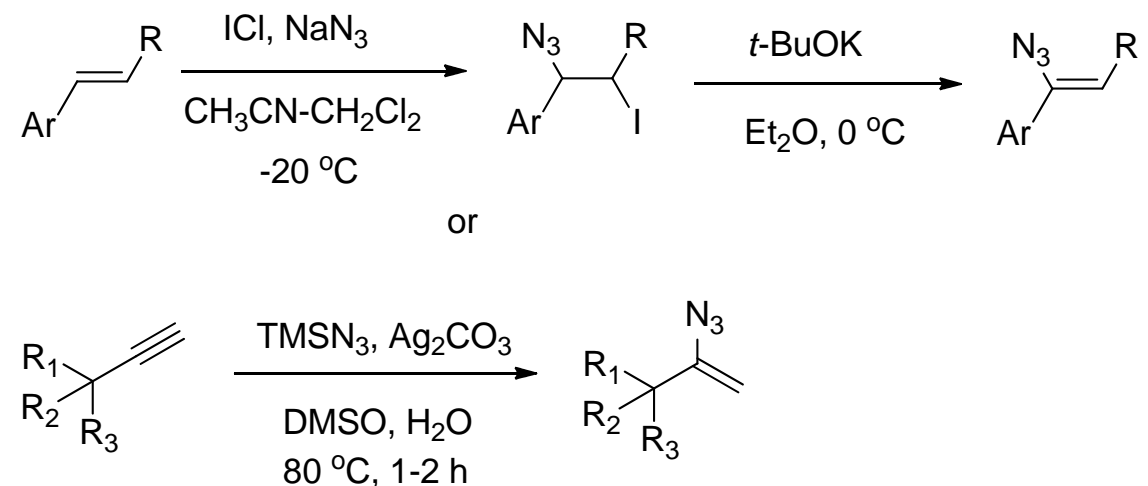

\section{Typical synthetic procedures:}

1: To a suspension of $\mathrm{NaN}_{3}(3.9 \mathrm{~g}, 60 \mathrm{mmol})$ in acetonitrile $(18 \mathrm{~mL})$ was added dropwise a solution of iodine monochloride $(5.8 \mathrm{~g}, 36 \mathrm{mmol})$ in $\mathrm{CH}_{2} \mathrm{Cl}_{2}(30 \mathrm{~mL})$ at $-20{ }^{\circ} \mathrm{C}$, and the mixture was stirred at the same temperature. After $30 \mathrm{~min}$, a solution of 4-vinylbiphenyl (4.3 g, $24 \mathrm{mmol})$ in $\mathrm{CH}_{2} \mathrm{Cl}_{2}(30 \mathrm{~mL})$ was added slowly, and the mixture was stirred for $1 \mathrm{~h}$. The reaction was quenched with saturated aqueous $\mathrm{Na}_{2} \mathrm{~S}_{2} \mathrm{O}_{3}$, and the organic materials were extracted two times with $\mathrm{Et}_{2} \mathrm{O}$. The combined extracts were washed with brine and dried over $\mathrm{MgSO}_{4}$. After evaporation of solvents, the resulting crude materials were used immediately for the next step without any further purification.

To a solution of the obtained compounds above in $\mathrm{Et}_{2} \mathrm{O}(60 \mathrm{~mL})$ was added $t$-BuOK (3.2 g, $28.8 \mathrm{mmol}$ ) at $0{ }^{\circ} \mathrm{C}$, and the mixture was stirred for $1.5 \mathrm{~h}$ at the same temperature. The reaction mixture was filtered through celite and the solvent was removed in vacuo. The resulting crude materials were purified by flash column chromatography (silica gel; hexane) to give 4-(1-azidovinyl)biphenyl (1a) (4.5 g, 
84\% yield ) as a white solid. (Angew. Chem., Int. Ed. 2014.53, 4390)

2: To a solution of (prop-2-ynyloxy) benzene (660 mg, $5 \mathrm{mmol}), \mathrm{TMSN}_{3}(1.15 \mathrm{~g}, 10$ mmol $)$ and $\mathrm{H}_{2} \mathrm{O}(0.18 \mathrm{~mL}, 10 \mathrm{mmol})$ in $\mathrm{DMSO}(8 \mathrm{~mL})$ at $80{ }^{\circ} \mathrm{C}, \mathrm{Ag}_{2} \mathrm{CO}_{3}(138 \mathrm{mg}, 0.5$ mmol) was added. The mixture was then stirred for 1-2 $\mathrm{h}$ until substrate (prop-2-ynyloxy) benzene consumed as indicated by TLC. The resulting mixture was concentrated and taken up by dichloromethane $(3 \times 30 \mathrm{~mL})$. The organic layer was washed with brine $(3 \times 40 \mathrm{~mL})$, dried over $\mathrm{MgSO}_{4}$ and concentrated. The resulting crude materials were purified by flash column chromatography (silica gel; hexane) to give (2-azidoallyloxy)benzene (1s) $(717 \mathrm{mg}, 82 \%$ yield ) as a yellow oil. (Org. Lett. 2014, 16, 3668)

\section{Typical experimental procedure}<smiles>C=C(N)c1ccc(-c2ccccc2)cc1</smiles>

To a suspension of Selectfluor (106.2 mg, $0.3 \mathrm{mmol}), \mathrm{NaHCO}_{3}(33.6 \mathrm{mg}, 0.4 \mathrm{mmol}$ ), and $\mathrm{H}_{2} \mathrm{O}(0.007 \mathrm{~mL}, 0.4 \mathrm{mmol})$ in $\mathrm{CH}_{3} \mathrm{CN}(2 \mathrm{~mL})$ was added vinyl azide 1a (44.2 $\mathrm{mg}$, $0.2 \mathrm{mmol}$ ) at $\mathrm{rt}$. The resulting mixture was stirred for $30 \mathrm{~min}$. The solvent was then removed under reduced pressure and the residue was purified by flash column chromatography on silica gel to give $2 \mathbf{a}$ as a white solid (35.4 mg, $82 \%$ yield).

\section{The reaction of $1 \mathrm{k}$}<smiles>C=C(N)c1ccccc1Br</smiles>

$1 \mathrm{k}$

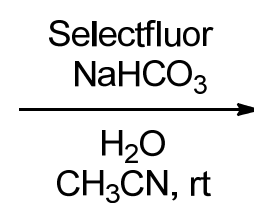

$\mathrm{CH}_{3} \mathrm{CN}$, rt<smiles>O=C(CF)c1ccccc1Br</smiles>

2k (41\%)<smiles>O=C(CF)Nc1ccccc1Br</smiles>

$3(15 \%)$

$N$-(2-Bromophenyl)-2-fluoroacetamide (3): ${ }^{1} \mathrm{H} \mathrm{NMR}\left(400 \mathrm{MHz}, \mathrm{CDCl}_{3}\right) \delta 8.56$ (brs, $1 \mathrm{H}), 8.38(\mathrm{~d}, J=8.2 \mathrm{~Hz}, 1 \mathrm{H}), 7.58(\mathrm{~d}, J=8.0 \mathrm{~Hz}, 1 \mathrm{H}), 7.35(\mathrm{t}, J=7.8 \mathrm{~Hz}, 1 \mathrm{H}), 7.04$ $(\mathrm{t}, J=7.6 \mathrm{~Hz}, 1 \mathrm{H}), 4.96(\mathrm{~d}, J=47.3 \mathrm{~Hz}, 2 \mathrm{H}) ;{ }^{13} \mathrm{C} \mathrm{NMR}\left(151 \mathrm{MHz}, \mathrm{CDCl}_{3}\right) \delta 165.7(\mathrm{~d}$, $\left.J_{C-F}=16.4 \mathrm{~Hz}\right), 134.6,132.6,128.6,126.1,122.0,113.8,80.4\left(\mathrm{~d}, J_{C-F}=188.4 \mathrm{~Hz}\right) ;{ }^{19} \mathrm{~F}$ NMR (564 MHz, $\left.\mathrm{CDCl}_{3}\right) \delta-217.80(\mathrm{td}, J=47.3,5.4 \mathrm{~Hz}, 1 \mathrm{~F})$. 


\section{References for known products}

\begin{tabular}{|l|l|l|}
\hline Entry & \multicolumn{1}{|c|}{ Reference } & Compound \\
\hline 1 & Q. Yang, ; L.-L. Mao. Org. Lett., 2014, 16, 3460. & $\begin{array}{l}\mathbf{2 b}, \mathbf{2 c}, \mathbf{2 d , ~ 2 e , ~ 2 i , ~} \\
\mathbf{2 j}, \mathbf{2 k , 2}, \mathbf{2}, \mathbf{2 f}, \mathbf{2 q}\end{array}$ \\
\hline 2 & T. Kitamura; K. Muta. J. Org. Chem., 2014, 79, 5842. & $\mathbf{2 m}$ \\
\hline 3 & W.-G. Wang; H.-S. Shen. J. Org. Chem., 2014, 79, 6347. & $\mathbf{2 p , ~ 2 r}$ \\
\hline 4 & T. Shao; X. Fang. Synlett, 2015, 26, 1835. & $\mathbf{2 a}$ \\
\hline 5 & E.D. Bergmann. J. Chem. Soc., 1961, 3448. & $\mathbf{2 s}$ \\
\hline 6 & R.P. Singh. J Fluorine chem., 2016, 181, 7. & $\mathbf{2 h}$ \\
\hline 7 & V. Pace. Tetrahedron, 2012, 53, 5106. & $\mathbf{2 w}$ \\
\hline 8 & $\begin{array}{l}\text { Merrell Toraude et Compagnie } \\
\text { Patent: US4134918A1, 1979. }\end{array}$ & $\mathbf{2 x}$ \\
\hline 9 & S. Sato; M. Yoshida. Synthesis, 2005, 15, 2602. & $\mathbf{2 y}$ \\
\hline
\end{tabular}




\section{Isotope labeling experiments}<smiles>C=C(N)c1ccc(-c2ccccc2)cc1</smiles>

\section{Elemental Composition Report}

Page 1

Multiple Mass Analysis: 2 mass(es) processed - displaying only valid results

Tolerance $=3.0 \mathrm{mDa} f$ OBE: $\min =-1.5, \max =50.0$

Element prediclion: Off

Monoisotopic Mass, Odd and Even Electron lons

4 formula(e) evaluated with 1 results vithin limits (all results (up to 1000) for each mass)

C: $0.14 \quad H: 0.19$ O: $0.1 \quad$ F: 0.1

SD22-WS-179 201 (3.350) Cm (198:201-(68:77+88:111))

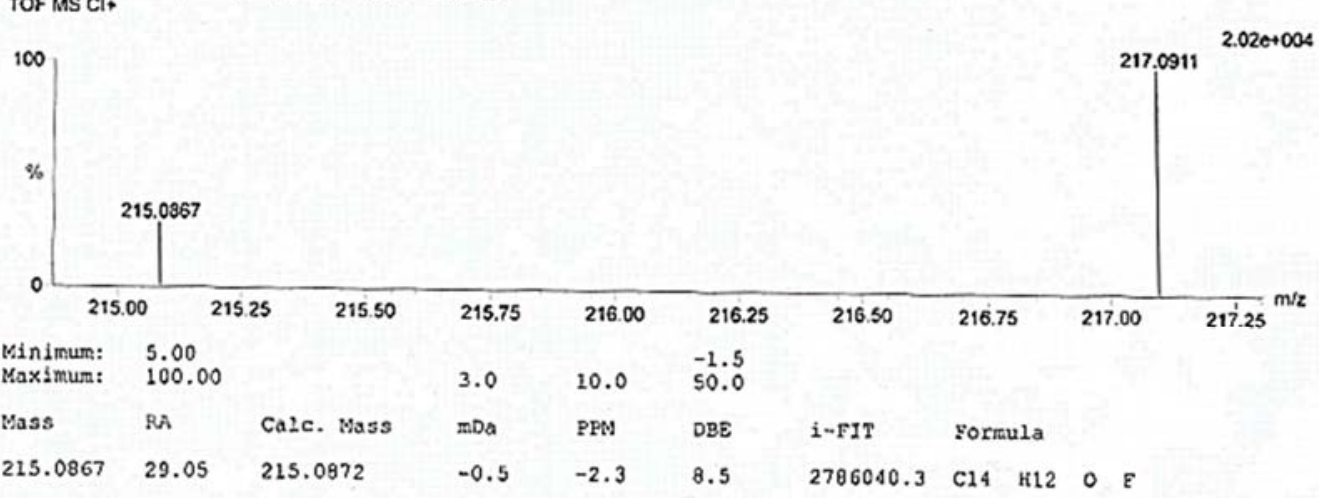

\section{Elemental Composition Report}

Multiple Mass Analysis: 2 mass(es) processed - displaying only valid results

Tolerance $=3.0 \mathrm{mDa} I \mathrm{DBE}: \min =-1.5, \max =50.0$

Element prediction: Off

Monoisotopic Mass, Odd and Even Electron lons

4 formula(e) evaluated with 1 results within limits (all results (up to 1000) for each mass)

Elements Used:

C: 0-14 H: 0-19 180: 0-1 F: 0-1

SD22-WS-179 201 (3.350) Cm (198:201-(68:77+88:111))

TOF MS Cl+

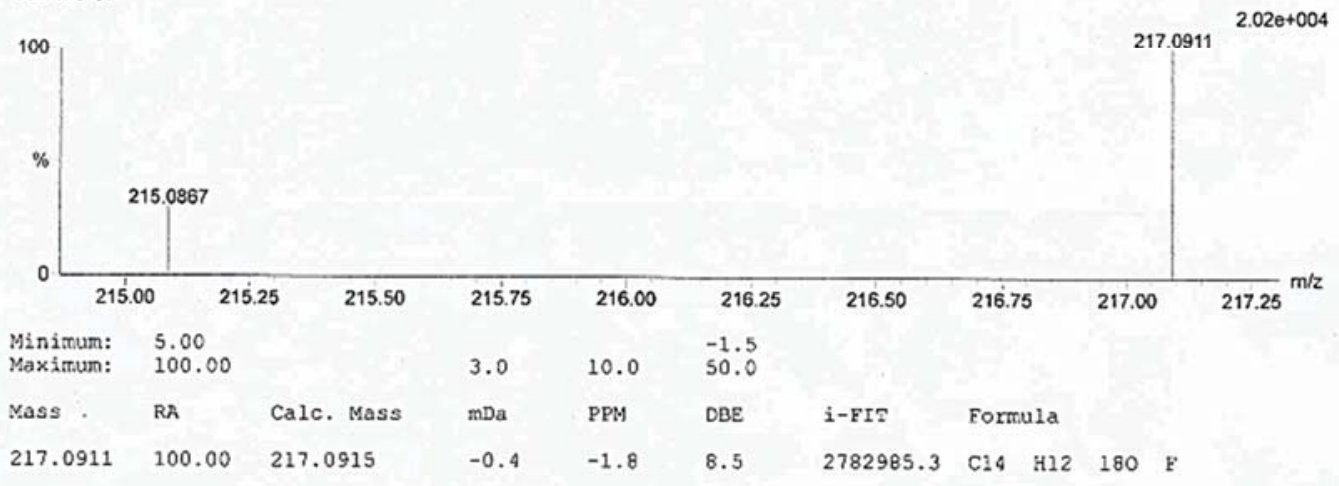


<smiles>NC(=Cc1ccc(-c2ccccc2)cc1)C(=O)OCC(=O)c1ccc(-c2ccc(C(=O)CF)cc2)cc1</smiles>

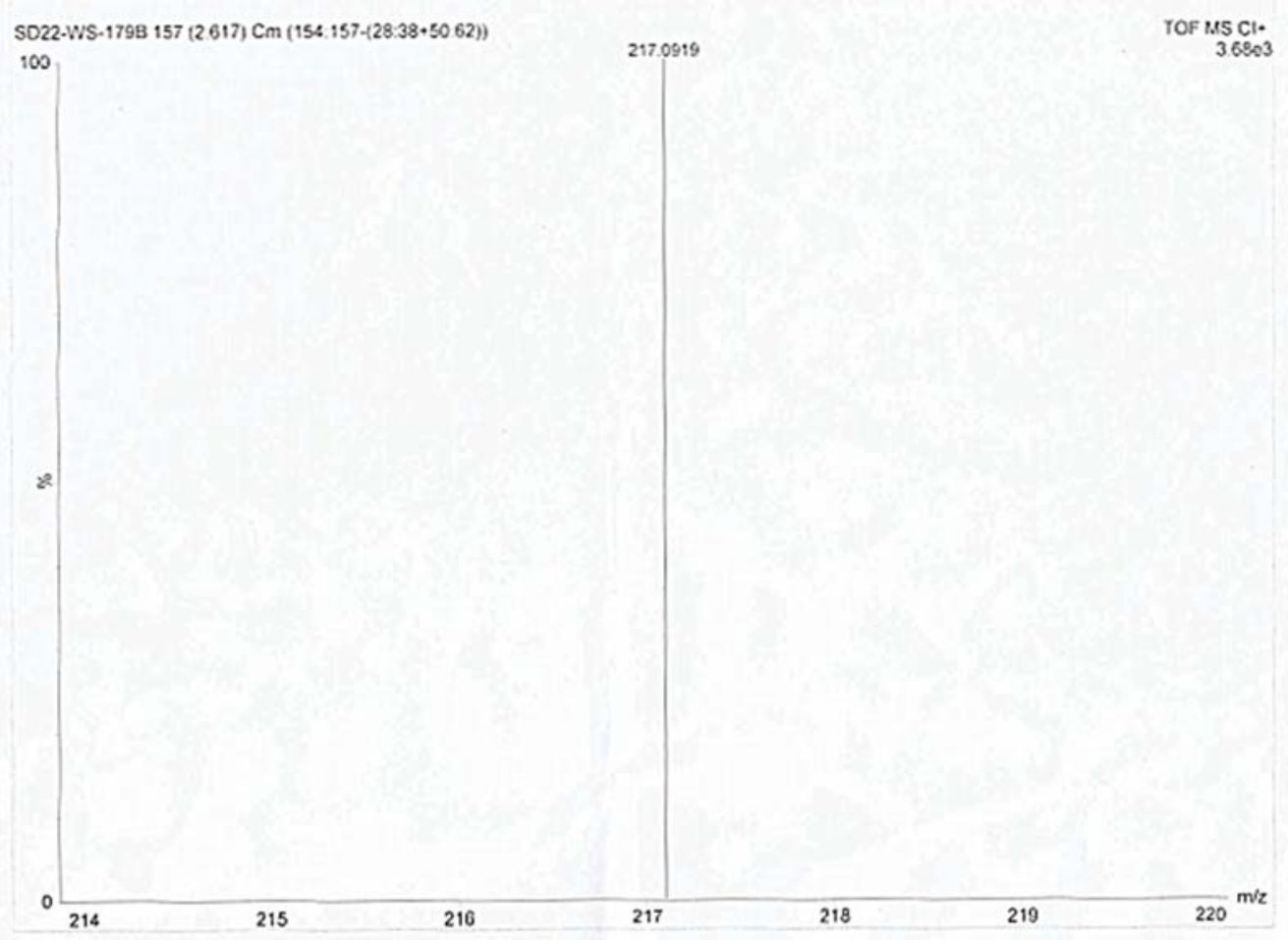

Elemental Composition Report

Page 1

Tolerance $=3.0 \mathrm{mDa} / \mathrm{DBE}: \min =-1.5, \max =50.0$

Element prediction: Off

Monoisolopic Mass, Odd and Even Electron lons

2 formula(e) evaluated with 1 results vithin limits (all results (up to 1000) for each mass)

Elements Used:

$\begin{array}{llll}C: 0-14 & \text { H: } 0-13 \quad \text { 180: } 0-1 \quad \text { F: } 0-1\end{array}$

SD22-WS-1798 157 (2.617) Cm (154:157-(13:29+57:82))

TOF MS Clt

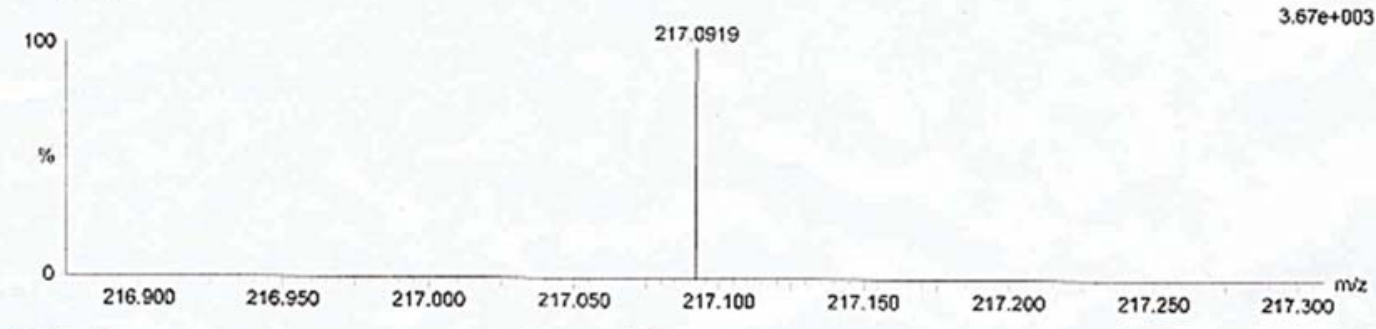

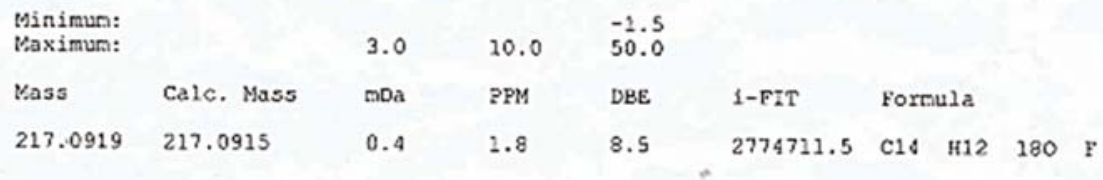




\section{Characterization of the substrates and products}<smiles>C=C(N)c1ccc(-c2ccccc2)cc1</smiles>

4-(1-Azidovinyl)biphenyl (1a): ${ }^{1} \mathrm{H}$ NMR $\left(400 \mathrm{MHz}, \mathrm{CDCl}_{3}\right) \delta 7.71-7.54(\mathrm{~m}, 6 \mathrm{H})$, $7.46(\mathrm{t}, J=7.5 \mathrm{~Hz}, 2 \mathrm{H}), 7.37(\mathrm{t}, J=7.2 \mathrm{~Hz}, 1 \mathrm{H}), 5.50(\mathrm{~d}, J=1.7 \mathrm{~Hz}, 1 \mathrm{H}), 5.00(\mathrm{~d}, J=$ $1.7 \mathrm{~Hz}, 1 \mathrm{H}) ;{ }^{13} \mathrm{C} \mathrm{NMR}\left(101 \mathrm{MHz}, \mathrm{CDCl}_{3}\right) \delta 144.9,142.0,140.5,133.3,129.0,127.8$, 127.3, 127.2, 126.1. 98.0.<smiles>C=C(N)c1ccccc1</smiles>

(1-Azidovinyl)benzene (1b): ${ }^{1} \mathrm{H} \mathrm{NMR}\left(600 \mathrm{MHz}, \mathrm{CDCl}_{3}\right) \delta 7.61-7.58(\mathrm{~m}, 2 \mathrm{H})$, $7.47-7.32(\mathrm{~m}, 3 \mathrm{H}), 5.46(\mathrm{~d}, J=2.3 \mathrm{~Hz}, 1 \mathrm{H}), 4.99(\mathrm{~d}, J=2.3 \mathrm{~Hz}, 1 \mathrm{H}) ;{ }^{13} \mathrm{C}$ NMR $(151$ $\left.\mathrm{MHz}, \mathrm{CDCl}_{3}\right) \delta 145.2,134.4,129.2,128.6,125.7,98.1$.<smiles>C=C(N)c1ccc(F)cc1</smiles>

1-(1-Azidovinyl)-4-fluorobenzene (1c): ${ }^{1} \mathrm{H} \mathrm{NMR}\left(400 \mathrm{MHz}, \mathrm{CDCl}_{3}\right) \delta 7.55$ (dd, $J=$ 8.5, $5.4 \mathrm{~Hz}, 2 \mathrm{H}), 7.04(\mathrm{t}, J=8.6 \mathrm{~Hz}, 2 \mathrm{H}), 5.38(\mathrm{~d}, J=2.1 \mathrm{~Hz}, 1 \mathrm{H}), 4.94(\mathrm{~d}, J=2.1 \mathrm{~Hz}$, $1 \mathrm{H}) ;{ }^{13} \mathrm{C} \mathrm{NMR}\left(101 \mathrm{MHz}, \mathrm{CDCl}_{3}\right) \delta 163.4\left(\mathrm{~d}, J_{C-F}=248.9 \mathrm{~Hz}\right), 144.2,130.5,127.5$ $\left(\mathrm{d}, J_{C-F}=8.3 \mathrm{~Hz}\right), 115.5\left(\mathrm{~d}, J_{C-F}=21.8 \mathrm{~Hz}\right), 97.7$.<smiles>C=C(N)c1ccc(Cl)cc1</smiles>

1-(1-Azidovinyl)-4-chlorobenzene (1d): ${ }^{1} \mathrm{H} \mathrm{NMR}\left(400 \mathrm{MHz}, \mathrm{CDCl}_{3}\right) \delta 7.50$ (d, $J=$ $8.5 \mathrm{~Hz}, 2 \mathrm{H}), 7.33$ (d, $J=8.5 \mathrm{~Hz}, 2 \mathrm{H}), 5.44(\mathrm{~d}, J=2.3 \mathrm{~Hz}, 1 \mathrm{H}), 4.98$ (d, $J=2.3 \mathrm{~Hz}$, $1 \mathrm{H}) ;{ }^{13} \mathrm{C}$ NMR $\left(101 \mathrm{MHz}, \mathrm{CDCl}_{3}\right) \delta 144.1,135.1,132.8,128.7,126.9,98.1$.<smiles>C=C(N)c1ccc(Br)cc1</smiles>

1-(1-Azidovinyl)-4-bromobenzene (1e): ${ }^{1} \mathrm{H} \mathrm{NMR}\left(400 \mathrm{MHz}, \mathrm{CDCl}_{3}\right) \delta 7.48$ (d, $J=$ $8.4 \mathrm{~Hz}, 2 \mathrm{H}), 7.43(\mathrm{~d}, J=8.4 \mathrm{~Hz}, 2 \mathrm{H}), 5.44(\mathrm{~d}, J=1.7 \mathrm{~Hz}, 1 \mathrm{H}), 4.98$ (d, $J=1.7 \mathrm{~Hz}$, $1 \mathrm{H}) ;{ }^{13} \mathrm{C} \mathrm{NMR}\left(101 \mathrm{MHz}, \mathrm{CDCl}_{3}\right) \delta 144.3,133.3,131.7,127.2,123.4,98.3$.<smiles>C=C(N)c1ccc(OC)cc1</smiles> 
1-(1-Azidovinyl)-4-methoxybenzene (1f): ${ }^{1} \mathrm{H} \mathrm{NMR}\left(400 \mathrm{MHz}, \mathrm{CDCl}_{3}\right) \delta 7.50$ (d, $J=$ $8.7 \mathrm{~Hz}, 2 \mathrm{H}), 6.88(\mathrm{~d}, J=8.7 \mathrm{~Hz}, 2 \mathrm{H}), 5.32(\mathrm{~d}, J=1.8 \mathrm{~Hz}, 1 \mathrm{H}), 4.87(\mathrm{~d}, J=1.7 \mathrm{~Hz}$, $1 \mathrm{H}), 3.83(\mathrm{~s}, 3 \mathrm{H}) ;{ }^{13} \mathrm{C} \mathrm{NMR}\left(151 \mathrm{MHz}, \mathrm{CDCl}_{3}\right.$, overlapping peaks) $\delta 160.5,144.8$, $127.0,113.9,96.3,55.4$.<smiles>C=C(N)c1ccc(C(C)=O)cc1</smiles>

1-(4-(1-Azidovinyl)phenyl)ethanone (1g): ${ }^{1} \mathrm{H} \mathrm{NMR}\left(400 \mathrm{MHz}, \mathrm{CDCl}_{3}\right) \delta 7.94$ (d, $J$ $=8.1 \mathrm{~Hz}, 2 \mathrm{H}), 7.65(\mathrm{~d}, J=8.1 \mathrm{~Hz}, 2 \mathrm{H}), 5.57(\mathrm{~d}, J=2.0 \mathrm{~Hz}, 1 \mathrm{H}), 5.07(\mathrm{~d}, J=1.6 \mathrm{~Hz}$, $1 \mathrm{H}), 2.60(\mathrm{~s}, 3 \mathrm{H}) ;{ }^{13} \mathrm{C} \mathrm{NMR}\left(151 \mathrm{MHz}, \mathrm{CDCl}_{3}\right) \delta 197.5,144.3,138.6,137.4,128.6$, 125.8, 99.9, 26.8 .<smiles>C=C(N)c1ccc([N+](=O)[O-])cc1</smiles>

1-(1-Azidovinyl)-4-nitrobenzene (1h): ${ }^{1} \mathrm{H}$ NMR (400 $\left.\mathrm{MHz}, \mathrm{CDCl}_{3}\right) \delta 8.20(\mathrm{~d}, J=$ $8.7 \mathrm{~Hz}, 2 \mathrm{H}), 7.73(\mathrm{~d}, J=8.7 \mathrm{~Hz}, 2 \mathrm{H}), 5.65(\mathrm{~d}, J=2.7 \mathrm{~Hz}, 1 \mathrm{H}), 5.16(\mathrm{~d}, J=2.7 \mathrm{~Hz}$, $1 \mathrm{H}) ;{ }^{13} \mathrm{C} \mathrm{NMR}\left(101 \mathrm{MHz}, \mathrm{CDCl}_{3}\right) \delta 148.1,143.4,140.2,126.4,123.8,101.4$.<smiles>C=C(N)c1cccc(Br)c1</smiles>

1-(1-Azidovinyl)-3-bromobenzene (1i): ${ }^{1} \mathrm{H}$ NMR (400 MHz, $\left.\mathrm{CDCl}_{3}\right) \delta 7.72(\mathrm{~s}, 1 \mathrm{H})$, 7.48 (t, $J=7.5 \mathrm{~Hz}, 2 \mathrm{H}), 7.23(\mathrm{q}, J=8.0 \mathrm{~Hz}, 1 \mathrm{H}), 5.46$ (d, $J=2.2 \mathrm{~Hz}, 1 \mathrm{H}), 4.99$ (d, $J=$ $2.2 \mathrm{~Hz}, 1 \mathrm{H}) ;{ }^{13} \mathrm{C} \mathrm{NMR}\left(101 \mathrm{MHz}, \mathrm{CDCl}_{3}\right) \delta 143.8,136.3,132.1,130.0,128.7,124.2$, $122.7,98.9$.<smiles>C=C(N)c1cccc(C)c1</smiles>

1-(1-Azidovinyl)-3-methylbenzene (1j): ${ }^{1} \mathrm{H} \mathrm{NMR}\left(400 \mathrm{MHz}, \mathrm{CDCl}_{3}\right) \delta 7.40-7.30$ (m, 2H), 7.23 (t, $J=7.5 \mathrm{~Hz}, 1 \mathrm{H}), 7.15$ (d, $J=7.4 \mathrm{~Hz}, 1 \mathrm{H}), 5.40$ (s, 1H), 4.93 (s, 1H), $2.35(\mathrm{~s}, 3 \mathrm{H}) ;{ }^{13} \mathrm{C} \mathrm{NMR}\left(151 \mathrm{MHz}, \mathrm{CDCl}_{3}\right) \delta 145.3,138.2,134.4,130.0,128.5,126.4$, $122.9,98.0,21.6$.<smiles>C=C(N)c1ccccc1Br</smiles>

1-(1-Azidovinyl)-2-bromobenzene (1k): ${ }^{1} \mathrm{H} \mathrm{NMR}\left(400 \mathrm{MHz}, \mathrm{CDCl}_{3}\right) \delta 7.63$ (d, $J=$ $7.9 \mathrm{~Hz}, 1 \mathrm{H}), 7.39-7.30(\mathrm{~m}, 2 \mathrm{H}), 7.29-7.21(\mathrm{~m}, 1 \mathrm{H}), 5.13(\mathrm{~s}, 1 \mathrm{H}), 4.86(\mathrm{~s}, 1 \mathrm{H}) ;{ }^{13} \mathrm{C}$ NMR $\left(101 \mathrm{MHz}, \mathrm{CDCl}_{3}\right) \delta 144.6,136.4,133.2,131.1,130.6,127.6,122.4,104.0$. 
<smiles>C=C(N)c1ccccc1OC</smiles>

1-(1-Azidovinyl)-2-methoxybenzene (11): ${ }^{1} \mathrm{H}$ NMR (400 MHz, $\left.\mathrm{CDCl}_{3}\right) \delta 7.40-7.32$ $(\mathrm{m}, 2 \mathrm{H}), 7.04-6.91(\mathrm{~m}, 2 \mathrm{H}), 5.05(\mathrm{~s}, 1 \mathrm{H}), 4.95(\mathrm{~s}, 1 \mathrm{H}), 3.90(\mathrm{~s}, 3 \mathrm{H}) ;{ }^{13} \mathrm{C}$ NMR $(151$ $\left.\mathrm{MHz}, \mathrm{CDCl}_{3}\right) \delta 156.8,143.1,130.7,130.4,123.7,120.8,111.0,103.1,55.7$.<smiles>C=C(N)c1ccc2ccccc2c1</smiles>

2-(1-Azidovinyl)naphthalene (1m): ${ }^{1} \mathrm{H}$ NMR (400 MHz, $\left.\mathrm{CDCl}_{3}\right) \delta 8.06(\mathrm{~s}, 1 \mathrm{H}), 7.92$ $-7.76(\mathrm{~m}, 3 \mathrm{H}), 7.68(\mathrm{~d}, J=8.6 \mathrm{~Hz}, 1 \mathrm{H}), 7.54-7.48(\mathrm{~m}, 2 \mathrm{H}), 5.60(\mathrm{~s}, 1 \mathrm{H}), 5.07$ (s, $1 \mathrm{H}) ;{ }^{13} \mathrm{C} \mathrm{NMR}\left(101 \mathrm{MHz}, \mathrm{CDCl}_{3}\right) \delta 145.1,133.7,133.2,131.6,128.7,128.3,127.7$, $126.8,126.6,125.1,123.2,98.4$.

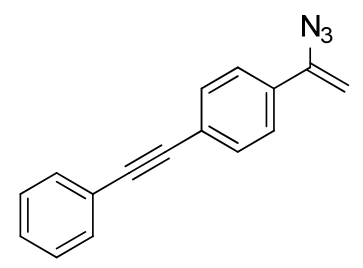

1-(1-Azidovinyl)-4-(phenylethynyl)benzene (1n): ${ }^{1} \mathrm{H}$ NMR $\left(400 \mathrm{MHz}, \mathrm{CDCl}_{3}\right) \delta$ $7.67-7.49(\mathrm{~m}, 6 \mathrm{H}), 7.46-7.31(\mathrm{~m}, 3 \mathrm{H}), 5.51(\mathrm{~d}, J=2.2 \mathrm{~Hz}, 1 \mathrm{H}), 5.02(\mathrm{~d}, J=2.1$ $\mathrm{Hz}, 1 \mathrm{H}) ;{ }^{13} \mathrm{C}$ NMR $\left(151 \mathrm{MHz}, \mathrm{CDCl}_{3}\right) \delta 144.5,133.9,131.7,131.7,128.5,128.5$, 125.5, 124.1, 123.2, 98.4, 90.9, 89.1.<smiles>C=CCCOC(=O)c1ccc(C(=C)N)cc1</smiles>

But-3-enyl 4-(1-azidovinyl)benzoate (1o): ${ }^{1} \mathrm{H}$ NMR (400 MHz, $\left.\mathrm{CDCl}_{3}\right) \delta 8.02$ (d, $J$ $=8.3 \mathrm{~Hz}, 2 \mathrm{H}), 7.63(\mathrm{~d}, J=8.3 \mathrm{~Hz}, 2 \mathrm{H}), 5.95-5.79(\mathrm{~m}, 1 \mathrm{H}), 5.56(\mathrm{~d}, J=2.2 \mathrm{~Hz}, 1 \mathrm{H})$, $5.14(\mathrm{dd}, J=24.6,13.7 \mathrm{~Hz}, 2 \mathrm{H}), 5.06(\mathrm{~d}, J=2.2 \mathrm{~Hz}, 1 \mathrm{H}), 4.38(\mathrm{t}, J=6.6 \mathrm{~Hz}, 2 \mathrm{H})$, $2.53(\mathrm{q}, J=6.5 \mathrm{~Hz}, 2 \mathrm{H}) ;{ }^{13} \mathrm{C}$ NMR $\left(151 \mathrm{MHz}, \mathrm{CDCl}_{3}\right) \delta 166.2,144.4,138.5,134.1$, $130.9,129.9,125.6,117.6,99.8,64.3,33.3$.<smiles>Nc1ccccc1N</smiles>

4-Azido-1,2-dihydronaphthalene (1p): ${ }^{1} \mathrm{H}$ NMR (400 $\left.\mathrm{MHz}, \mathrm{CDCl}_{3}\right) \delta 7.43-7.33$ (m, 1H), $7.24-7.18(\mathrm{~m}, 2 \mathrm{H}), 7.15(\mathrm{~d}, J=4.1 \mathrm{~Hz}, 1 \mathrm{H}), 5.72(\mathrm{t}, J=4.7 \mathrm{~Hz}, 1 \mathrm{H}), 2.83$ (t, $J=8.0 \mathrm{~Hz}, 2 \mathrm{H}), 2.46(\mathrm{dt}, J=12.6,6.5 \mathrm{~Hz}, 2 \mathrm{H}) ;{ }^{13} \mathrm{C} \mathrm{NMR}\left(151 \mathrm{MHz}, \mathrm{CDCl}_{3}\right) \delta 136.4$, 136.0, 130.5, 128.3, 127.6, 126.7, 122.3, 111.9, 27.8, 22.8. 


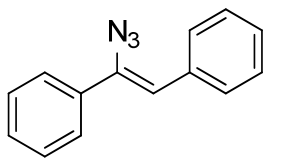

(Z)-(1-Azidoethene-1,2-diyl)dibenzene (1q): ${ }^{1} \mathrm{H}$ NMR $\left(400 \mathrm{MHz}, \mathrm{CDCl}_{3}\right) \delta 7.88(\mathrm{~d}$, $J=7.7 \mathrm{~Hz}, 2 \mathrm{H}), 7.74-7.47(\mathrm{~m}, 7 \mathrm{H}), 7.44-7.36(\mathrm{~m}, 1 \mathrm{H}), 6.17(\mathrm{~s}, 1 \mathrm{H}) ;{ }^{13} \mathrm{C} \mathrm{NMR}$ $\left(101 \mathrm{MHz}, \mathrm{CDCl}_{3}\right) \delta 136.5,135.8,135.2,129.1,128.8,128.4,127.4,127.3,118.5$, 118.4 .<smiles>C/C=C(\N)c1ccc(OC)cc1</smiles>

(Z)-1-(1-Azidoprop-1-en-1-yl)-4-methoxybenzene (1r): ${ }^{1} \mathrm{H}$ NMR (400 $\mathrm{MHz}, \mathrm{CDCl}_{3}$ ) $\delta 7.25(\mathrm{~d}, J=8.5 \mathrm{~Hz}, 2 \mathrm{H}), 6.91(\mathrm{~d}, J=8.5 \mathrm{~Hz}, 2 \mathrm{H}), 5.41$ (q, $J=7.2 \mathrm{~Hz}, 1 \mathrm{H}), 3.79$ (s, $3 \mathrm{H}), 1.69(\mathrm{~d}, J=7.2 \mathrm{~Hz}, 3 \mathrm{H}) ;{ }^{13} \mathrm{C} \mathrm{NMR}\left(101 \mathrm{MHz}, \mathrm{CDCl}_{3}\right) \delta 159.7,137.1,130.1$, $125.6,113.8,111.3,55.2,14.0$.<smiles>C=C(N)COc1ccccc1</smiles>

(2-Azidoallyloxy)benzene (1s): ${ }^{1} \mathrm{H} \mathrm{NMR}\left(400 \mathrm{MHz}, \mathrm{CDCl}_{3}\right) \delta 7.33(\mathrm{t}, J=7.8 \mathrm{~Hz}$, 2H), $7.10-6.89(\mathrm{~m}, 3 \mathrm{H}), 5.07(\mathrm{~s}, 1 \mathrm{H}), 4.93(\mathrm{~s}, 1 \mathrm{H}), 4.48(\mathrm{~s}, 2 \mathrm{H}) ;{ }^{13} \mathrm{C}$ NMR $(101 \mathrm{MHz}$, $\left.\mathrm{CDCl}_{3}\right) \delta 158.0,142.2,129.6,121.6,115.0,101.1,67.7$.<smiles>C=C(N)COc1ccc(C=O)cc1</smiles>

4-(2-Azidoallyloxy)benzaldehyde (1t): ${ }^{1} \mathrm{H} \mathrm{NMR}\left(400 \mathrm{MHz}, \mathrm{CDCl}_{3}\right) \delta 9.89(\mathrm{~s}, 1 \mathrm{H})$, $7.84(\mathrm{~d}, J=8.5 \mathrm{~Hz}, 2 \mathrm{H}), 7.03(\mathrm{~d}, J=8.5 \mathrm{~Hz}, 2 \mathrm{H}), 5.09(\mathrm{~s}, 1 \mathrm{H}), 4.95(\mathrm{~s}, 1 \mathrm{H}), 4.51$ (s, $2 \mathrm{H}) ;{ }^{13} \mathrm{C} \mathrm{NMR}\left(101 \mathrm{MHz}, \mathrm{CDCl}_{3}\right) \delta 190.8,162.9,141.5,132.1,130.7,115.2,101.5$, 67.8 .<smiles>C=C(N)COc1ccc(C(C)O)cc1</smiles>

4-(2-Azidoallyloxy)phenyl)ethanol (1u): ${ }^{1} \mathrm{H}$ NMR (400 MHz, $\left.\mathrm{CDCl}_{3}\right) \delta 7.29$ (d, $J=$ $8.3 \mathrm{~Hz}, 2 \mathrm{H}), 6.90(\mathrm{~d}, J=8.4 \mathrm{~Hz}, 2 \mathrm{H}), 5.03(\mathrm{~s}, 1 \mathrm{H}), 4.90(\mathrm{~s}, 1 \mathrm{H}), 4.84(\mathrm{q}, J=6.2 \mathrm{~Hz}$, $1 \mathrm{H}), 4.44(\mathrm{~s}, 2 \mathrm{H}), 1.99(\mathrm{~s}, 1 \mathrm{H}), 1.47(\mathrm{~d}, J=6.4 \mathrm{~Hz}, 3 \mathrm{H}) ;{ }^{13} \mathrm{C} \mathrm{NMR}\left(151 \mathrm{MHz}, \mathrm{CDCl}_{3}\right)$ $\delta 157.4,142.1,139.0,126.8,115.0,101.1,70.00,67.9,25.1$.<smiles>C=C(N)CN[As]</smiles> 
$\mathbf{N}$-(2-Azidoallyl)-4-methylbenzenesulfonamide (1v): ${ }^{1} \mathrm{H}$ NMR (400 $\left.\mathrm{MHz}, \mathrm{CDCl}_{3}\right) \delta$ $7.74(\mathrm{~d}, J=8.0 \mathrm{~Hz}, 2 \mathrm{H}), 7.29(\mathrm{~d}, J=8.0 \mathrm{~Hz}, 2 \mathrm{H}), 5.21(\mathrm{t}, J=6.1 \mathrm{~Hz}, 1 \mathrm{H}), 4.87$ (s, $1 \mathrm{H}), 4.62(\mathrm{~s}, 1 \mathrm{H}), 3.51(\mathrm{~d}, J=6.4 \mathrm{~Hz}, 2 \mathrm{H}), 2.41(\mathrm{~s}, 3 \mathrm{H}) ;{ }^{13} \mathrm{C} \mathrm{NMR}\left(151 \mathrm{MHz}, \mathrm{CDCl}_{3}\right)$ $\delta 143.8,142.2,137.0,129.7,127.3,100.4,45.4,21.6$.<smiles>C=C(N)CN1C(=O)c2ccccc2C1=O</smiles>

2-(2-Azidoallyl)isoindoline-1,3-dione (1w): ${ }^{1} \mathrm{H} \mathrm{NMR}\left(400 \mathrm{MHz}, \mathrm{CDCl}_{3}\right) \delta 7.88(\mathrm{dd}$, $J=5.0,3.1 \mathrm{~Hz}, 2 \mathrm{H}), 7.74(\mathrm{dd}, J=5.1,3.1 \mathrm{~Hz}, 2 \mathrm{H}), 4.92(\mathrm{~d}, J=67.7 \mathrm{~Hz}, 2 \mathrm{H}), 4.24(\mathrm{~s}$, $2 \mathrm{H}) ;{ }^{13} \mathrm{C} \mathrm{NMR}\left(151 \mathrm{MHz}, \mathrm{CDCl}_{3}\right) \delta 167.5,141.4,134.2,131.9,123.5,100.0,39.6$.<smiles>C=C(N)CCCN1C(=O)c2ccccc2C1=O</smiles>

2-(4-Azidopent-4-enyl)isoindoline-1,3-dione (1x): ${ }^{1} \mathrm{H}$ NMR $\left(600 \mathrm{MHz}, \mathrm{CDCl}_{3}\right) \delta$ $7.85-7.78(\mathrm{~m}, 2 \mathrm{H}), 7.73-7.64(\mathrm{~m}, 2 \mathrm{H}), 4.72(\mathrm{~d}, J=1.1 \mathrm{~Hz}, 1 \mathrm{H}), 4.63(\mathrm{~d}, J=1.1 \mathrm{~Hz}$, $1 \mathrm{H}), 3.68(\mathrm{t}, J=7.1 \mathrm{~Hz}, 2 \mathrm{H}), 2.10(\mathrm{t}, J=7.5 \mathrm{~Hz}, 2 \mathrm{H}), 1.90-1.82(\mathrm{~m}, 2 \mathrm{H}) ;{ }^{13} \mathrm{C} \mathrm{NMR}$ $\left(151 \mathrm{MHz}, \mathrm{CDCl}_{3}\right) \delta 168.4,145.7,134.0,132.1,123.3,98.6,37.3,31.3,26.2$.

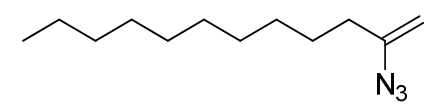

2-Azidododec-1-ene (1y): ${ }^{1} \mathrm{H}$ NMR $\left(400 \mathrm{MHz}, \mathrm{CDCl}_{3}\right) \delta 4.62$ (s, 2H), 2.07 (t, $J=7.5$ $\mathrm{Hz}, 2 \mathrm{H}), 1.54-1.38(\mathrm{~m}, 2 \mathrm{H}), 1.35-1.22(\mathrm{~m}, 14 \mathrm{H}), 0.88(\mathrm{t}, J=6.5 \mathrm{~Hz}, 3 \mathrm{H}) ;{ }^{13} \mathrm{C}$ NMR (151 MHz, $\left.\mathrm{CDCl}_{3}\right) \delta 147.0,98.1,33.8,32.1,29.8,29.7,29.52,29.49,29.0$, $27.5,22.9,14.2$.<smiles>N/C(=C\C1CC1)c1ccc(-c2ccccc2)cc1</smiles>

4-(1-Azido-2-cyclopropylvinyl)biphenyl (1z): ${ }^{1} \mathrm{H}$ NMR $\left(400 \mathrm{MHz}, \mathrm{CDCl}_{3}\right) \delta 7.66-$ $7.54(\mathrm{~m}, 4 \mathrm{H}), 7.51-7.40(\mathrm{~m}, 4 \mathrm{H}), 7.36(\mathrm{t}, J=7.2 \mathrm{~Hz}, 1 \mathrm{H}), 4.76(\mathrm{~d}, J=9.6 \mathrm{~Hz}, 1 \mathrm{H})$, $2.00-1.77(\mathrm{~m}, 1 \mathrm{H}), 0.99-0.81(\mathrm{~m}, 2 \mathrm{H}), 0.62-0.39(\mathrm{~m}, 2 \mathrm{H}) ;{ }^{13} \mathrm{C}$ NMR $(101 \mathrm{MHz}$, $\left.\mathrm{CDCl}_{3}\right) \delta 141.2,140.5,135.2,134.1,129.0,127.7,127.4,127.1,127.0,125.3,10.5$, 7.8 .<smiles>O=C(CF)c1ccc(-c2ccccc2)cc1</smiles> 
1-(Biphenyl-4-yl)-2-fluoroethanone (2a): White solid; m.p. 120-122 ${ }^{\circ} \mathrm{C} ; 82 \%$ yield $(35 \mathrm{mg}) ;{ }^{1} \mathrm{H}$ NMR $\left(400 \mathrm{MHz}, \mathrm{CDCl}_{3}\right) \delta 7.98(\mathrm{~d}, J=8.1 \mathrm{~Hz}, 2 \mathrm{H}), 7.72(\mathrm{~d}, J=8.1 \mathrm{~Hz}$, 2H), 7.63 (d, $J=7.4 \mathrm{~Hz}, 2 \mathrm{H}), 7.49$ (t, $J=7.3 \mathrm{~Hz}, 2 \mathrm{H}), 7.46-7.39$ (m, 1H), 5.56 (d, $J$ $=46.9 \mathrm{~Hz}, 2 \mathrm{H}) ;{ }^{13} \mathrm{C} \mathrm{NMR}\left(101 \mathrm{MHz}, \mathrm{CDCl}_{3}\right) \delta 193.2\left(\mathrm{~d}, J_{C-F}=15.6 \mathrm{~Hz}\right), 147.0$, $139.7,132.5,129.2,128.64\left(\mathrm{~d}, J_{C-F}=0.5 \mathrm{~Hz}\right), 128.61,127.6,127.4,83.8\left(\mathrm{~d}, J_{C-F}=\right.$ $182.7 \mathrm{~Hz}) ;{ }^{19} \mathrm{~F}$ NMR $\left(376 \mathrm{MHz}, \mathrm{CDCl}_{3}\right) \delta-230.33(\mathrm{t}, J=47.0 \mathrm{~Hz}, 1 \mathrm{~F})$.<smiles>O=C(CF)c1ccccc1</smiles>

2-Fluoro-1-phenylethanone (2b): Yellow oil; 77\% yield (21 mg); ${ }^{1} \mathrm{H}$ NMR (400 $\left.\mathrm{MHz}, \mathrm{CDCl}_{3}\right) \delta 7.89(\mathrm{~d}, J=7.7 \mathrm{~Hz}, 2 \mathrm{H}), 7.63(\mathrm{t}, J=7.3 \mathrm{~Hz}, 1 \mathrm{H}), 7.50(\mathrm{t}, J=7.6 \mathrm{~Hz}$, $2 \mathrm{H}), 5.54(\mathrm{~d}, J=46.9 \mathrm{~Hz}, 2 \mathrm{H}) ;{ }^{13} \mathrm{C} \mathrm{NMR}\left(101 \mathrm{MHz}, \mathrm{CDCl}_{3}\right) \delta 193.5\left(\mathrm{~d}, J_{C-F}=15.5\right.$ $\mathrm{Hz}), 134.3,133.8,129.1,128.0\left(\mathrm{~d}, J_{C-F}=2.6 \mathrm{~Hz}\right), 83.7$ (d, $\left.J_{C-F}=182.6 \mathrm{~Hz}\right) ;{ }^{19} \mathrm{~F} \mathrm{NMR}$ $\left(376 \mathrm{MHz}, \mathrm{CDCl}_{3}\right) \delta-230.79(\mathrm{t}, J=46.9 \mathrm{~Hz}, 1 \mathrm{~F})$.<smiles>O=C(CF)c1ccc(F)cc1</smiles>

2-Fluoro-1-(4-fluorophenyl)ethanone (2c): White solid; m.p. 50-52 ${ }^{\circ} \mathrm{C}$; 71\% yield $(22 \mathrm{mg}) ;{ }^{1} \mathrm{H}$ NMR $\left(400 \mathrm{MHz}, \mathrm{CDCl}_{3}\right) \delta 7.94(\mathrm{dd}, J=8.1,5.6 \mathrm{~Hz}, 2 \mathrm{H}), 7.16(\mathrm{t}, J=8.5$ $\mathrm{Hz}, 2 \mathrm{H}), 5.48(\mathrm{~d}, J=46.9 \mathrm{~Hz}, 2 \mathrm{H}) ;{ }^{13} \mathrm{C} \mathrm{NMR}\left(101 \mathrm{MHz}, \mathrm{CDCl}_{3}\right) \delta 192.2(\mathrm{~d}, J=16.0$ $\mathrm{Hz}), 166.4\left(\mathrm{~d}, J_{C-F}=256.5 \mathrm{~Hz}\right), 130.9\left(\mathrm{dd}, J_{C-F}=9.5,3.1 \mathrm{~Hz}\right), 130.3\left(\mathrm{~d}, J_{C-F}=2.3 \mathrm{~Hz}\right)$, $116.3\left(\mathrm{~d}, J_{C-F}=22.1 \mathrm{~Hz}\right), 83.7\left(\mathrm{~d}, J_{C-F}=183.1 \mathrm{~Hz}\right) ;{ }^{19} \mathrm{~F}$ NMR $\left(376 \mathrm{MHz}, \mathrm{CDCl}_{3}\right) \delta$ $-102.79--102.82(\mathrm{~m}, 1 \mathrm{~F}),-229.53(\mathrm{t}, J=46.9 \mathrm{~Hz}, 1 \mathrm{~F})$.<smiles>O=C(CF)c1ccc(Cl)cc1</smiles>

1-(4-Chlorophenyl)-2-fluoroethanone (2d): White solid; m.p. 46-48 ${ }^{\circ} \mathrm{C}$; 83\% yield $(28 \mathrm{mg}) ;{ }^{1} \mathrm{H} \mathrm{NMR}\left(400 \mathrm{MHz}, \mathrm{CDCl}_{3}\right) \delta 7.86(\mathrm{~d}, J=8.4 \mathrm{~Hz}, 2 \mathrm{H}), 7.48(\mathrm{~d}, J=8.4 \mathrm{~Hz}$, $2 \mathrm{H}), 5.48(\mathrm{~d}, J=46.9 \mathrm{~Hz}, 2 \mathrm{H}) ;{ }^{13} \mathrm{C} \mathrm{NMR}\left(151 \mathrm{MHz}, \mathrm{CDCl}_{3}\right) \delta 192.6\left(\mathrm{~d}, J_{C-F}=16.1\right.$ $\mathrm{Hz}), 140.8,132.2,129.6\left(\mathrm{~d}, J_{C-F}=2.9 \mathrm{~Hz}\right), 129.5,83.8\left(\mathrm{~d}, J_{C-F}=183.6 \mathrm{~Hz}\right) ;{ }^{19} \mathrm{~F} \mathrm{NMR}$ $\left(376 \mathrm{MHz}, \mathrm{CDCl}_{3}\right) \delta-229.57(\mathrm{t}, J=46.9 \mathrm{~Hz}, 1 \mathrm{~F})$.<smiles>O=C(CF)c1ccc(Br)cc1</smiles>

1-(4-Bromophenyl)-2-fluoroethanone (2e): White solid; m.p.71-73 ${ }^{\circ} \mathrm{C}$; $74 \%$ yield (32 mg); ${ }^{1} \mathrm{H}$ NMR (400 MHz, $\left.\mathrm{CDCl}_{3}\right) \delta 7.76(\mathrm{~d}, J=7.1 \mathrm{~Hz}, 2 \mathrm{H}), 7.64(\mathrm{~d}, J=7.9 \mathrm{~Hz}$, 2H), $5.47(\mathrm{~d}, J=46.9 \mathrm{~Hz}, 2 \mathrm{H}) ;{ }^{13} \mathrm{C} \mathrm{NMR}\left(101 \mathrm{MHz}, \mathrm{CDCl}_{3}\right) \delta 192.8\left(\mathrm{~d}, J_{\mathrm{C}-\mathrm{F}}=16.1\right.$ $\mathrm{Hz}), 132.6,132.4,129.58,129.55,83.7$ (d, $\left.J_{C-F}=183.5 \mathrm{~Hz}\right) ;{ }^{19} \mathrm{~F}$ NMR $(376 \mathrm{MHz}$, $\left.\mathrm{CDCl}_{3}\right) \delta-229.75(\mathrm{t}, J=46.9 \mathrm{~Hz}, 1 \mathrm{~F})$. 
<smiles>COc1ccc(C(=O)CF)cc1</smiles>

2-Fluoro-1-(4-methoxyphenyl)ethanone (2f): White solid; m.p.80-82 ${ }^{\circ} \mathrm{C}$; $82 \%$ yield $(28 \mathrm{mg}) ;{ }^{1} \mathrm{H}$ NMR $\left(400 \mathrm{MHz}, \mathrm{CDCl}_{3}\right) \delta 7.89(\mathrm{~d}, J=8.6 \mathrm{~Hz}, 2 \mathrm{H}), 6.96(\mathrm{~d}, J=8.7 \mathrm{~Hz}$, 2H), $5.48(\mathrm{~d}, J=47.0 \mathrm{~Hz}, 2 \mathrm{H}), 3.88(\mathrm{~s}, 3 \mathrm{H}) ;{ }^{13} \mathrm{C} \mathrm{NMR}\left(151 \mathrm{MHz}, \mathrm{CDCl}_{3}\right) \delta 192.1(\mathrm{~d}$, $\left.J_{C-F}=15.6 \mathrm{~Hz}\right), 164.4,130.43\left(\mathrm{~d}, J_{C-F}=2.7 \mathrm{~Hz}\right), 126.9,114.3,83.6\left(\mathrm{~d}, J_{C-F}=182.0\right.$ $\mathrm{Hz}), 55.7 ;{ }^{19} \mathrm{~F} \mathrm{NMR}\left(376 \mathrm{MHz}, \mathrm{CDCl}_{3}\right) \delta-229.83(\mathrm{t}, J=47.0 \mathrm{~Hz}, 1 \mathrm{~F})$.<smiles>CC(=O)c1ccc(C(=O)CF)cc1</smiles>

1-(4-Acetylphenyl)-2-fluoroethanone (2g): Yellowish solid; m.p. 121-123 ${ }^{\circ} \mathrm{C} ; 72 \%$ yield (26 mg); ${ }^{1} \mathrm{H} \mathrm{NMR}\left(400 \mathrm{MHz}, \mathrm{CDCl}_{3}\right) \delta 8.05(\mathrm{~d}, J=8.2 \mathrm{~Hz}, 2 \mathrm{H}), 7.98(\mathrm{~d}, J=8.2$ $\mathrm{Hz}, 2 \mathrm{H}), 5.53(\mathrm{~d}, J=46.8 \mathrm{~Hz}, 2 \mathrm{H}), 2.65(\mathrm{~s}, 3 \mathrm{H}) ;{ }^{13} \mathrm{C} \mathrm{NMR}\left(151 \mathrm{MHz}, \mathrm{CDCl}_{3}\right) \delta 197.3$, $193.3\left(\mathrm{~d}, J_{C-F}=16.1 \mathrm{~Hz}\right), 141.0,136.9,128.8,128.3\left(\mathrm{~d}, J_{C-F}=2.5 \mathrm{~Hz}\right), 83.8\left(\mathrm{~d}, J_{C-F}=\right.$ $183.8 \mathrm{~Hz}), 27.0 ;{ }^{19} \mathrm{~F}$ NMR $\left(564 \mathrm{MHz}, \mathrm{CDCl}_{3}\right) \delta-225.24--225.46(\mathrm{~m}, 1 \mathrm{~F})$; FT-IR (thin film, KBr): $v$ (cm-1) 2964, 2924, 1699, 1558, 829; HRMS (CI) calcd $\mathrm{C}_{10} \mathrm{H}_{10} \mathrm{FO}_{2}$ $[\mathrm{M}+\mathrm{H}]^{+}:$181.0665, found: 181.0661 .<smiles>O=C(CF)c1ccc([N+](=O)[O-])cc1</smiles>

2-Fluoro-1-(4-nitrophenyl)ethanone (2h): Yellow solid; m.p. 97-99 ${ }^{\circ} \mathrm{C}$; 70\% yield $(26 \mathrm{mg}) ;{ }^{1} \mathrm{H}$ NMR $\left(400 \mathrm{MHz}, \mathrm{CDCl}_{3}\right) \delta 8.35(\mathrm{~d}, J=8.5 \mathrm{~Hz}, 2 \mathrm{H}), 8.09(\mathrm{~d}, J=8.5 \mathrm{~Hz}$, $2 \mathrm{H}), 5.53(\mathrm{~d}, J=46.8 \mathrm{~Hz}, 2 \mathrm{H}) ;{ }^{13} \mathrm{C} \mathrm{NMR}\left(151 \mathrm{MHz}, \mathrm{CDCl}_{3}\right) \delta 192.7\left(\mathrm{~d}, J_{C-F}=16.8\right.$ $\mathrm{Hz}), 150.9,138.3,129.4\left(\mathrm{~d}, J_{C-F}=3.2 \mathrm{~Hz}\right), 124.2,84.0\left(\mathrm{~d}, J_{C-F}=184.9 \mathrm{~Hz}\right) ;{ }^{19} \mathrm{~F} \mathrm{NMR}$ $\left(564 \mathrm{MHz}, \mathrm{CDCl}_{3}\right) \delta-228.93(\mathrm{t}, J=46.8 \mathrm{~Hz}, 1 \mathrm{~F})$.<smiles>O=C(CF)c1cccc(Br)c1</smiles>

1-(3-Bromophenyl)-2-fluoroethanone (2i): Colorless oil; 72\% yield $(31 \mathrm{mg}) ;{ }^{1} \mathrm{H}$ NMR (400 MHz, $\left.\mathrm{CDCl}_{3}\right) \delta 8.02(\mathrm{~s}, 1 \mathrm{H}), 7.81(\mathrm{~d}, J=7.7 \mathrm{~Hz}, 1 \mathrm{H}), 7.74(\mathrm{~d}, J=7.9 \mathrm{~Hz}$, $1 \mathrm{H}), 7.38(\mathrm{t}, J=7.9 \mathrm{~Hz}, 1 \mathrm{H}), 5.48(\mathrm{~d}, J=46.8 \mathrm{~Hz}, 2 \mathrm{H}) ;{ }^{13} \mathrm{C} \mathrm{NMR}\left(101 \mathrm{MHz}, \mathrm{CDCl}_{3}\right)$ $\delta 192.4\left(\mathrm{~d}, J_{C-F}=16.0 \mathrm{~Hz}\right), 137.1,135.5,131.1\left(\mathrm{~d}, J_{C-F}=2.9 \mathrm{~Hz}\right), 130.6,126.6\left(\mathrm{~d}, J_{C-F}\right.$ $=2.9 \mathrm{~Hz}), 123.4,83.6\left(\mathrm{~d}, J_{\mathrm{C}-\mathrm{F}}=183.7 \mathrm{~Hz}\right) ;{ }^{19} \mathrm{~F} \mathrm{NMR}\left(376 \mathrm{MHz}, \mathrm{CDCl}_{3}\right) \delta-230.08(\mathrm{t}$, $J=46.8 \mathrm{~Hz}, 1 \mathrm{~F})$. 
<smiles>Cc1cccc(C(=O)CF)c1</smiles>

2-Fluoro-1-(m-tolyl)ethanone (2j): Colorless oil; 74\% yield (22 mg); ${ }^{1} \mathrm{H}$ NMR (400 $\left.\mathrm{MHz}, \mathrm{CDCl}_{3}\right) \delta 7.69(\mathrm{~s}, 1 \mathrm{H}), 7.65(\mathrm{~d}, J=7.6 \mathrm{~Hz}, 1 \mathrm{H}), 7.42(\mathrm{~d}, J=7.4 \mathrm{~Hz}, 1 \mathrm{H}), 7.37$ $(\mathrm{d}, J=7.6 \mathrm{~Hz}, 1 \mathrm{H}), 5.51(\mathrm{~d}, J=46.9 \mathrm{~Hz}, 2 \mathrm{H}), 2.40(\mathrm{~s}, 3 \mathrm{H}) ;{ }^{13} \mathrm{C}$ NMR $(151 \mathrm{MHz}$, $\left.\mathrm{CDC}_{3}\right) \delta 193.6\left(\mathrm{~d}, J_{C-F}=15.3 \mathrm{~Hz}\right), 138.9,135.0,133.8,128.9,128.3\left(\mathrm{~d}, J_{C-F}=2.0 \mathrm{~Hz}\right)$, $125.0\left(\mathrm{~d}, J_{C-F}=2.2 \mathrm{~Hz}\right), 83.6\left(\mathrm{~d}, J_{C-F}=182.2 \mathrm{~Hz}\right), 21.4 ;{ }^{19} \mathrm{~F} \mathrm{NMR}\left(564 \mathrm{MHz}, \mathrm{CDCl}_{3}\right)$ $\delta-231.06(\mathrm{t}, J=46.9 \mathrm{~Hz}, 1 \mathrm{~F})$.<smiles>O=C(CF)c1ccccc1Br</smiles>

1-(2-Bromophenyl)-2-fluoroethanone (2k): Colorless oil; $41 \%$ yield (18 mg); ${ }^{1} \mathrm{H}$ NMR (400 MHz, $\left.\mathrm{CDCl}_{3}\right) \delta 7.65(\mathrm{~d}, J=7.7 \mathrm{~Hz}, 1 \mathrm{H}), 7.48$ (d, J=7.3 Hz, 1H), $7.45-$ $7.33(\mathrm{~m}, 2 \mathrm{H}), 5.37$ (d, $J=47.2 \mathrm{~Hz}, 2 \mathrm{H}) ;{ }^{13} \mathrm{C} \mathrm{NMR}\left(151 \mathrm{MHz}, \mathrm{CDCl}_{3}\right) \delta 197.9\left(\mathrm{~d}, J_{C-F}\right.$ $=18.8 \mathrm{~Hz}), 137.6,133.9,133.0,129.7,127.7,119.6,84.3\left(\mathrm{~d}, J_{C-F}=186.6 \mathrm{~Hz}\right) ;{ }^{19} \mathrm{~F}$ $\mathrm{NMR}\left(564 \mathrm{MHz}, \mathrm{CDCl}_{3}\right) \delta-219.83(\mathrm{t}, J=47.2 \mathrm{~Hz}, 1 \mathrm{~F})$.<smiles>COc1ccccc1C(=O)CF</smiles>

2-Fluoro-1-(2-methoxyphenyl)ethanone (2l): White solid; m.p. 89-91 ${ }^{\circ} \mathrm{C} ; 63 \%$ yield $(21 \mathrm{mg}) ;{ }^{1} \mathrm{H}$ NMR $\left(400 \mathrm{MHz}, \mathrm{CDCl}_{3}\right) \delta 7.99(\mathrm{~d}, J=7.4 \mathrm{~Hz}, 1 \mathrm{H}), 7.53(\mathrm{t}, J=7.3$ $\mathrm{Hz}, 1 \mathrm{H}), 7.05$ (t, $J=7.5 \mathrm{~Hz}, 1 \mathrm{H}), 6.99$ (d, $J=8.4 \mathrm{~Hz}, 1 \mathrm{H}), 5.40$ (d, $J=48.2 \mathrm{~Hz}, 2 \mathrm{H})$, $3.93(\mathrm{~s}, 3 \mathrm{H}) ;{ }^{13} \mathrm{C}$ NMR $\left(151 \mathrm{MHz}, \mathrm{CDCl}_{3}\right) \delta 193.9\left(\mathrm{~d}, J_{C-F}=14.2 \mathrm{~Hz}\right), 159.7,135.3$, $130.9\left(\mathrm{~d}, J_{C-F}=1.9 \mathrm{~Hz}\right), 123.7,121.2,111.6,86.4\left(\mathrm{~d}, J_{C-F}=179.4 \mathrm{~Hz}\right), 55.7 ;{ }^{19} \mathrm{~F} \mathrm{NMR}$ $\left(564 \mathrm{MHz}, \mathrm{CDCl}_{3}\right) \delta-223.99(\mathrm{t}, J=48.2 \mathrm{~Hz}, 1 \mathrm{~F})$.<smiles>O=C(CF)c1ccc2ccccc2c1</smiles>

2-Fluoro-1-(naphthalen-2-yl)ethanone (2m): White solid; m.p. 85-87 ${ }^{\circ} \mathrm{C} ; 88 \%$ yield (33 mg); ${ }^{1} \mathrm{H}$ NMR (400 MHz, $\left.\mathrm{CDCl}_{3}\right) \delta 8.40(\mathrm{~s}, 1 \mathrm{H}), 8.01-7.81(\mathrm{~m}, 4 \mathrm{H}), 7.64$ $(\mathrm{t}, J=7.3 \mathrm{~Hz}, 1 \mathrm{H}), 7.58(\mathrm{t}, J=7.4 \mathrm{~Hz}, 1 \mathrm{H}), 5.66(\mathrm{~d}, J=46.9 \mathrm{~Hz}, 2 \mathrm{H}) ;{ }^{13} \mathrm{C}$ NMR $(101$ $\left.\mathrm{MHz}_{2} \mathrm{CDCl}_{3}\right) \delta 193.5\left(\mathrm{~d}, J_{C-F}=15.5 \mathrm{~Hz}\right), 136.1,132.5,131.1,129.9\left(\mathrm{~d}, J_{C-F}=3.2\right.$ $\mathrm{Hz}), 129.7,129.2,129.0,128.0,127.3,123.3\left(\mathrm{~d}, J_{C-F}=2.1 \mathrm{~Hz}\right), 83.8\left(\mathrm{~d}, J_{C-F}=182.6\right.$ $\mathrm{Hz}) ;{ }^{19} \mathrm{~F} \mathrm{NMR}\left(376 \mathrm{MHz}, \mathrm{CDCl}_{3}\right) \delta-230.11$ (t, $\left.J=46.9 \mathrm{~Hz}, 1 \mathrm{~F}\right)$. 


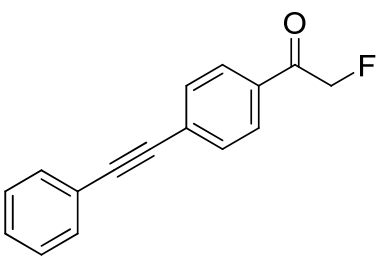

2-Fluoro-1-(4-(phenylethynyl)phenyl)ethanone (2n): White solid; m.p. 124-126 ${ }^{\circ} \mathrm{C}$; $80 \%$ yield $(38 \mathrm{mg}) ;{ }^{1} \mathrm{H}$ NMR $\left(400 \mathrm{MHz}, \mathrm{CDCl}_{3}\right) \delta 7.87(\mathrm{~d}, J=8.2 \mathrm{~Hz}, 2 \mathrm{H}), 7.63(\mathrm{~d}, J$ $=8.2 \mathrm{~Hz}, 2 \mathrm{H}), 7.59-7.47(\mathrm{~m}, 2 \mathrm{H}), 7.38(\mathrm{~d}, J=2.2 \mathrm{~Hz}, 3 \mathrm{H}), 5.51(\mathrm{~d}, J=46.8 \mathrm{~Hz}, 2 \mathrm{H})$; ${ }^{13} \mathrm{C} \mathrm{NMR}\left(151 \mathrm{MHz}, \mathrm{CDCl}_{3}\right) \delta 192.8\left(\mathrm{~d}, J_{C-F}=15.6 \mathrm{~Hz}\right), 132.8,132.0,131.9,129.4$, 129.1, 128.6, $128.0\left(\mathrm{~d}, J_{C-F}=2.5 \mathrm{~Hz}\right), 122.5,93.6,88.5,83.7\left(\mathrm{~d}, J_{C-F}=183.0 \mathrm{~Hz}\right) ;{ }^{19} \mathrm{~F}$ NMR (564 MHz, $\left.\mathrm{CDCl}_{3}\right) \delta-230.36(\mathrm{t}, J=46.8 \mathrm{~Hz}, 1 \mathrm{~F})$; FT-IR (thin Film, KBr): $v$ $\left(\mathrm{cm}^{-1}\right)$ 2933, 2218, 1699, 1591, 829; HRMS (CI) calcd $\mathrm{C}_{16} \mathrm{H}_{12} \mathrm{FO}[\mathrm{M}+\mathrm{H}]^{+}: 239.0872$, found: 239.0867.<smiles>C=CCCOC(=O)c1ccc(C(=O)CF)cc1</smiles>

Allyl 4-(2-fluoroacetyl)benzoate (2o): White solid; m.p. 49-51 ${ }^{\circ} \mathrm{C} ; 77 \%$ yield $(42$ mg; ${ }^{1} \mathrm{H}$ NMR (400 MHz, $\left.\mathrm{CDCl}_{3}\right) \delta 8.12(\mathrm{~d}, J=8.2 \mathrm{~Hz}, 2 \mathrm{H}), 7.93(\mathrm{~d}, J=8.2 \mathrm{~Hz}, 2 \mathrm{H})$, $5.91-5.79(\mathrm{~m}, 1 \mathrm{H}), 5.53(\mathrm{~d}, J=46.8 \mathrm{~Hz}, 2 \mathrm{H}), 5.13(\mathrm{dd}, J=23.3,13.7 \mathrm{~Hz}, 2 \mathrm{H}), 4.39$ (t, $J=6.6 \mathrm{~Hz}, 2 \mathrm{H}), 2.53(\mathrm{q}, J=6.5 \mathrm{~Hz}, 2 \mathrm{H}) ;{ }^{13} \mathrm{C} \mathrm{NMR}\left(151 \mathrm{MHz}, \mathrm{CDCl}_{3}\right) \delta 193.3(\mathrm{~d}$, $\left.J_{C-F}=15.9 \mathrm{~Hz}\right), 165.4,136.9,135.1,133.8,130.1,128.0\left(\mathrm{~d}, J_{C-F}=2.5 \mathrm{~Hz}\right), 117.7,83.8$ $\left(\mathrm{d}, J_{C-F}=183.6 \mathrm{~Hz}\right), 64.6,33.2 ;{ }^{19} \mathrm{~F}$ NMR $\left(564 \mathrm{MHz} \mathrm{CDCl}_{3}\right) \delta-230.27(\mathrm{t}, J=46.8$ $\mathrm{Hz}, 1 \mathrm{~F}$ ); FT-IR (thin film, KBr): $v\left(\mathrm{~cm}^{-1}\right)$ 2929, 2112, 1721, 1267, 855; HRMS (CI) calcd $\mathrm{C}_{13} \mathrm{H}_{14} \mathrm{FO}_{3}[\mathrm{M}+\mathrm{H}]^{+}$: 237.0927, found: 237.0922 .<smiles>O=C1c2ccccc2CCC1F</smiles>

2-Fluoro-3,4-dihydronaphthalen-1(2H)-one (2p): White solid; m.p. 37-39 ${ }^{\circ} \mathrm{C} ; 87 \%$ yield $(29 \mathrm{mg}) ;{ }^{1} \mathrm{H}$ NMR $\left(400 \mathrm{MHz}, \mathrm{CDCl}_{3}\right) \delta 8.04(\mathrm{~d}, J=7.7 \mathrm{~Hz}, 1 \mathrm{H}), 7.52(\mathrm{t}, J=7.4$ $\mathrm{Hz}, 1 \mathrm{H}), 7.34(\mathrm{t}, J=7.4 \mathrm{~Hz}, 1 \mathrm{H}), 7.27(\mathrm{~d}, J=7.5 \mathrm{~Hz}, 1 \mathrm{H}), 5.25-5.03(\mathrm{~m}, 1 \mathrm{H}), 3.34-$ $3.03(\mathrm{~m}, 2 \mathrm{H}), 2.79-2.46(\mathrm{~m}, 1 \mathrm{H}), 2.46-2.17(\mathrm{~m}, 1 \mathrm{H}) ;{ }^{13} \mathrm{C} \mathrm{NMR}\left(151 \mathrm{MHz}, \mathrm{CDCl}_{3}\right)$ $\delta 193.4\left(\mathrm{~d}, J_{C-F}=14.6 \mathrm{~Hz}\right), 143.1,134.2,131.2,128.7,127.8\left(\mathrm{~d}, J_{C-F}=1.9 \mathrm{~Hz}\right), 127.1$, $91.2\left(\mathrm{~d}, J_{C-F}=187.9 \mathrm{~Hz}\right), 30.2\left(\mathrm{~d}, J_{C-F}=19.1 \mathrm{~Hz}\right), 27.0\left(\mathrm{~d}, J_{C-F}=11.5 \mathrm{~Hz}\right) ;{ }^{19} \mathrm{~F} \mathrm{NMR}$ $\left(564 \mathrm{MHz}, \mathrm{CDCl}_{3}\right) \delta-187.79--195.52(\mathrm{~m}, 1 \mathrm{~F})$.<smiles>O=C(c1ccccc1)C(F)c1ccccc1</smiles>

2-Fluoro-1,2-diphenylethanone (2q): Yellow oil; 87\% yield (37 mg); ${ }^{1} \mathrm{H}$ NMR (400 $\left.\mathrm{MHz}, \mathrm{CDCl}_{3}\right) \delta 7.94(\mathrm{~d}, J=7.7 \mathrm{~Hz}, 2 \mathrm{H}), 7.55(\mathrm{t}, J=7.4 \mathrm{~Hz}, 1 \mathrm{H}), 7.52-7.45(\mathrm{~m}, 2 \mathrm{H})$, $7.44-7.39(\mathrm{~m}, 5 \mathrm{H}), 6.52(\mathrm{~d}, J=48.6 \mathrm{~Hz}, 1 \mathrm{H}) ;{ }^{13} \mathrm{C} \mathrm{NMR}\left(151 \mathrm{MHz}, \mathrm{CDCl}_{3}\right) \delta 194.4$ 
$\left(\mathrm{d}, J_{C-F}=21.4 \mathrm{~Hz}\right), 134.5,134.3,134.2,133.9,129.8\left(\mathrm{~d}, J_{C-F}=2.3 \mathrm{~Hz}\right), 129.2,128.8$, $127.5\left(\mathrm{~d}, J_{C-F}=5.4 \mathrm{~Hz}\right), 94.1\left(\mathrm{~d}, J_{C-F}=185.7 \mathrm{~Hz}\right) ;{ }^{19} \mathrm{~F} \mathrm{NMR}\left(564 \mathrm{MHz}, \mathrm{CDCl}_{3}\right) \delta$ $-173.37(\mathrm{~d}, J=48.6 \mathrm{~Hz}, 1 \mathrm{~F})$.<smiles>COc1ccc(C(=O)C(C)F)cc1</smiles>

2-Fluoro-1-(4-methoxyphenyl)propan-1-one (2r): Colorless oil; 81\% yield (29 mg); ${ }^{1} \mathrm{H}$ NMR (400 MHz, CDCl 3 ) $\delta 7.96(\mathrm{~d}, J=8.6 \mathrm{~Hz}, 2 \mathrm{H}), 6.94(\mathrm{~d}, J=8.7 \mathrm{~Hz}, 2 \mathrm{H}), 5.65$ $(\mathrm{dq}, J=48.8,6.8 \mathrm{~Hz}, 1 \mathrm{H}), 3.86(\mathrm{~s}, 3 \mathrm{H}), 1.63(\mathrm{dd}, J=24.1,6.8 \mathrm{~Hz}, 3 \mathrm{H}) ;{ }^{13} \mathrm{C} \mathrm{NMR}$ $\left(151 \mathrm{MHz}, \mathrm{CDCl}_{3}\right) \delta 195.4\left(\mathrm{~d}, J_{C-F}=19.2 \mathrm{~Hz}\right), 164.1,131.5\left(\mathrm{~d}, J_{C-F}=3.9 \mathrm{~Hz}\right), 127.0$, 114.0, $90.4\left(\mathrm{~d}, J_{C-F}=179.6 \mathrm{~Hz}\right), 55.6,18.6\left(\mathrm{~d}, J_{C-F}=22.8 \mathrm{~Hz}\right) ;{ }^{19} \mathrm{~F}$ NMR $(564 \mathrm{MHz}$, $\left.\mathrm{CDCl}_{3}\right) \delta-180.67--180.45(\mathrm{~m}, 1 \mathrm{~F})$.<smiles>O=C(CF)COc1ccccc1</smiles>

1-Fluoro-3-phenoxypropan-2-one (2s): Yellowish oil; 66\% yield (22 mg); ${ }^{1} \mathrm{H}$ NMR $\left(400 \mathrm{MHz}, \mathrm{CDCl}_{3}\right) \delta 7.32(\mathrm{t}, J=7.8 \mathrm{~Hz}, 2 \mathrm{H}), 7.03(\mathrm{t}, J=7.3 \mathrm{~Hz}, 1 \mathrm{H}), 6.90$ (d, $J=8.1$ $\mathrm{Hz}, 2 \mathrm{H}), 5.20(\mathrm{~d}, J=47.2 \mathrm{~Hz}, 2 \mathrm{H}), 4.80(\mathrm{~s}, 2 \mathrm{H}) ;{ }^{13} \mathrm{C} \mathrm{NMR}\left(101 \mathrm{MHz}, \mathrm{CDCl}_{3}\right) \delta 201.8$ $\left(\mathrm{d}, J_{C-F}=17.3 \mathrm{~Hz}\right), 157.5,129.9,122.3,114.6,84.6\left(\mathrm{~d}, J_{C-F}=182.5 \mathrm{~Hz}\right), 71.4\left(\mathrm{~d}, J_{C-F}\right.$ $=1.4 \mathrm{~Hz}) ;{ }^{19} \mathrm{~F}$ NMR $\left(376 \mathrm{MHz}, \mathrm{CDCl}_{3}\right) \delta-236.38(\mathrm{t}, J=47.1 \mathrm{~Hz}, 1 \mathrm{~F})$.<smiles>O=Cc1ccc(OCC(=O)CF)cc1</smiles>

4-(3-Fluoro-2-oxopropoxy)benzaldehyde (2t): Yellow solid; m.p. 77-79 ${ }^{\circ} \mathrm{C} ; 61 \%$ yield (24 mg); ${ }^{1} \mathrm{H}$ NMR (400 MHz, $\left.\mathrm{CDCl}_{3}\right) \delta 9.88(\mathrm{~s}, 1 \mathrm{H}), 7.84(\mathrm{~d}, J=8.5 \mathrm{~Hz}, 2 \mathrm{H})$, $7.00(\mathrm{~d}, J=8.5 \mathrm{~Hz}, 2 \mathrm{H}), 5.16(\mathrm{~d}, J=47.1 \mathrm{~Hz}, 2 \mathrm{H}), 4.94(\mathrm{~s}, 2 \mathrm{H}) ;{ }^{13} \mathrm{C}$ NMR $(101 \mathrm{MHz}$, $\left.\mathrm{CDCl}_{3}\right) \delta 200.63\left(\mathrm{~d}, J_{C-F}=18.8 \mathrm{~Hz}\right), 190.8,162.2,132.2,131.1,114.9,84.6\left(\mathrm{~d}, J_{C-F}=\right.$ $182.6 \mathrm{~Hz}), 71.0\left(\mathrm{~d}, J_{C-F}=1.9 \mathrm{~Hz}\right) ;{ }^{19} \mathrm{~F}$ NMR $\left(376 \mathrm{MHz}, \mathrm{CDCl}_{3}\right) \delta-236.02(\mathrm{t}, J=47.1$ $\mathrm{Hz}, 1 \mathrm{~F}$ ); FT-IR (thin film, KBr): $v\left(\mathrm{~cm}^{-1}\right) 3169,1667,1597,1465,829$; HRMS (CI) calcd $\mathrm{C}_{10} \mathrm{H}_{10} \mathrm{FO}_{3}[\mathrm{M}+\mathrm{H}]^{+}$: 197.0614, found:197.0610.<smiles>CC(O)c1ccc(OCC(=O)CF)cc1</smiles>

1-Fluoro-3-(4-(1-hydroxyethyl)phenoxy)propan-2-one (2u): yellow oil; 58\% yield $(25 \mathrm{mg}) ;{ }^{1} \mathrm{H}$ NMR $\left(400 \mathrm{MHz}, \mathrm{CDCl}_{3}\right) \delta 7.33(\mathrm{~d}, J=8.3 \mathrm{~Hz}, 2 \mathrm{H}), 6.88(\mathrm{~d}, J=8.3 \mathrm{~Hz}$, $2 \mathrm{H}), 5.19(\mathrm{~d}, J=47.2 \mathrm{~Hz}, 2 \mathrm{H}), 4.92-4.83(\mathrm{~m}, 1 \mathrm{H}), 4.80(\mathrm{~s}, 2 \mathrm{H}), 1.83(\mathrm{~s}, 1 \mathrm{H}), 1.48(\mathrm{~d}$, $J=6.3 \mathrm{~Hz}, 3 \mathrm{H}) ;{ }^{13} \mathrm{C} \mathrm{NMR}\left(151 \mathrm{MHz}, \mathrm{CDCl}_{3}\right) \delta 201.8\left(\mathrm{~d}, J_{C-F}=17.6 \mathrm{~Hz}\right), 156.9$, $139.8,127.1,114.6,84.6\left(\mathrm{~d}, J_{C-F}=182.5 \mathrm{~Hz}\right), 71.5,69.9,25.3 ;{ }^{19} \mathrm{~F}$ NMR $(564 \mathrm{MHz}$, 
$\left.\mathrm{CDCl}_{3}\right) \delta-236.41(\mathrm{t}, J=47.1 \mathrm{~Hz}, 1 \mathrm{~F})$; FT-IR (thin film, $\left.\mathrm{KBr}\right): v\left(\mathrm{~cm}^{-1}\right) 3392,2972$, 2931, 1611, 829; HRMS (CI) calcd $\mathrm{C}_{11} \mathrm{H}_{13} \mathrm{FO}_{3}$ [M] : 212.0849, found: 212.0847.<smiles>[3H]NCC(=O)CF</smiles>

$\boldsymbol{N}$-(3-fluoro-2-oxopropyl)-4-methylbenzenesulfonamide (2v): White solid; m.p. 112- $114{ }^{\circ} \mathrm{C}$; 61\% yield (30 mg); ${ }^{1} \mathrm{H}$ NMR $\left(400 \mathrm{MHz}, \mathrm{CDCl}_{3}\right) \delta 7.75(\mathrm{~d}, J=8.0 \mathrm{~Hz}$, 2H), $7.32(\mathrm{~d}, J=7.9 \mathrm{~Hz}, 2 \mathrm{H}), 5.31(\mathrm{~s}, 1 \mathrm{H}), 4.86(\mathrm{~d}, J=47.1 \mathrm{~Hz}, 2 \mathrm{H}), 4.09$ (d, $J=4.0$ $\mathrm{Hz}, 2 \mathrm{H}), 2.43(\mathrm{~s}, 3 \mathrm{H}) ;{ }^{13} \mathrm{C} \mathrm{NMR}\left(151 \mathrm{MHz}, \mathrm{CDCl}_{3}\right) \delta 201.2\left(\mathrm{~d}, J_{C-F}=22.5 \mathrm{~Hz}\right), 144.2$, $136.05,130.0,127.3,84.4\left(\mathrm{~d}, J_{C-F}=183.3 \mathrm{~Hz}\right), 49.4\left(\mathrm{~d}, J_{C-F}=2.6 \mathrm{~Hz}\right), 21.7 ;{ }^{19} \mathrm{~F} \mathrm{NMR}$ $\left(564 \mathrm{MHz}, \mathrm{CDCl}_{3}\right) \delta-234.08(\mathrm{t}, J=47.1 \mathrm{~Hz}, 1 \mathrm{~F})$; FT-IR (thin film, $\left.\mathrm{KBr}\right): v\left(\mathrm{~cm}^{-1}\right)$ 3273, 2925, 1749, 1439, 1270; HRMS (CI) calcd $\mathrm{C}_{10} \mathrm{H}_{13} \mathrm{FNO}_{3} \mathrm{~S}[\mathrm{M}+\mathrm{H}]^{+}: 246.0600$, found: 246.0594 .<smiles>O=C(CF)CN1C(=O)c2ccccc2C1=O</smiles>

2-(3-Fluoro-2-oxopropyl)isoindoline-1,3-dione( 2w): White solid; m.p. 99-101 ${ }^{\circ} \mathrm{C}$; $65 \%$ yield $(29 \mathrm{mg}) ;{ }^{1} \mathrm{H}$ NMR $\left(400 \mathrm{MHz}, \mathrm{CDCl}_{3}\right) \delta 7.97-7.83(\mathrm{~m}, 2 \mathrm{H}), 7.83-7.69$ (m, 2H), $5.01(\mathrm{~d}, J=47.2 \mathrm{~Hz}, 2 \mathrm{H}), 4.75(\mathrm{~s}, 2 \mathrm{H}) ;{ }^{13} \mathrm{C} \mathrm{NMR}\left(151 \mathrm{MHz}, \mathrm{CDCl}_{3}\right) \delta 199.4$ $\left(\mathrm{d}, J_{C-F}=22.5 \mathrm{~Hz}\right), 167.6,134.4,132.1,123.7,85.0\left(\mathrm{~d}, J_{C-F}=182.9 \mathrm{~Hz}\right), 44.5\left(\mathrm{~d}, J_{C-F}\right.$ $=2.6 \mathrm{~Hz}) ;{ }^{19} \mathrm{~F} \mathrm{NMR}\left(564 \mathrm{MHz}, \mathrm{CDCl}_{3}\right) \delta-233.39(\mathrm{tt}, J=47.1,1.8 \mathrm{~Hz}, 1 \mathrm{~F})$.<smiles>O=C(CF)CCCN1C(=O)c2ccccc2C1=O</smiles>

2-(5-Fluoro-4-oxopentyl)isoindoline-1,3-dione (2x): White solid; m.p. 91-93 ${ }^{\circ} \mathrm{C}$; $70 \%$ yield (35 mg); ${ }^{1} \mathrm{H}$ NMR (400 $\left.\mathrm{MHz}, \mathrm{CDCl}_{3}\right) \delta 7.80(\mathrm{dd}, J=5.1,3.1 \mathrm{~Hz}, 2 \mathrm{H}), 7.69$ $(\mathrm{dd}, J=5.1,3.1 \mathrm{~Hz}, 2 \mathrm{H}), 4.79(\mathrm{~d}, J=47.6 \mathrm{~Hz}, 2 \mathrm{H}), 3.70(\mathrm{t}, J=6.5 \mathrm{~Hz}, 2 \mathrm{H}), 2.58$ (t, $J$ $=6.1 \mathrm{~Hz}, 2 \mathrm{H}), 1.98(\mathrm{p}, J=6.7 \mathrm{~Hz}, 2 \mathrm{H}) ;{ }^{13} \mathrm{C} \mathrm{NMR}\left(151 \mathrm{MHz}, \mathrm{CDCl}_{3}\right) \delta 205.8\left(\mathrm{~d}, J_{\mathrm{C}-\mathrm{F}}\right.$ $=19.6 \mathrm{~Hz}), 168.5,134.1,132.0,123.3,85.0\left(\mathrm{~d}, J_{C-F}=184.5 \mathrm{~Hz}\right), 37.0,35.2,21.7 ;{ }^{19} \mathrm{~F}$ NMR $\left(564 \mathrm{MHz}, \mathrm{CDCl}_{3}\right) \delta-228.05(\mathrm{tt}, J=47.4,2.1 \mathrm{~Hz}, 1 \mathrm{~F})$.

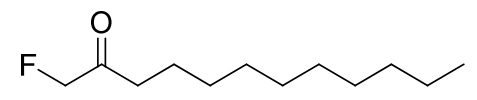

1-Fluorododecan-2-one (2y): Colorless oil; 76\% yield (31 mg); ${ }^{1} \mathrm{H}$ NMR (400 MHz, $\left.\mathrm{CDCl}_{3}\right) \delta 4.77(\mathrm{~d}, J=47.7 \mathrm{~Hz}, 2 \mathrm{H}), 2.51(\mathrm{td}, J=7.3,2.1 \mathrm{~Hz}, 2 \mathrm{H}), 1.66-1.52(\mathrm{~m}, 2 \mathrm{H})$, $1.27-1.24(\mathrm{~m}, 14 \mathrm{H}), 0.86(\mathrm{t}, J=6.5 \mathrm{~Hz}, 3 \mathrm{H}) ;{ }^{13} \mathrm{C} \mathrm{NMR}\left(151 \mathrm{MHz}, \mathrm{CDCl}_{3}\right) \delta 207.2$ $\left(\mathrm{d}, J_{C-F}=19.2 \mathrm{~Hz}\right), 85.0\left(\mathrm{~d}, J_{C-F}=184.9 \mathrm{~Hz}\right), 38.4,32.0,29.7,29.5,29.44,29.40,29.2$, 22.9, 22.8, 14.2; ${ }^{19} \mathrm{~F}$ NMR (564 MHz, $\left.\mathrm{CDCl}_{3}\right) \delta-227.58(\mathrm{tt}, J=47.7,2.7 \mathrm{~Hz}, 1 \mathrm{~F})$. 
<smiles>O=C(c1ccc(-c2ccccc2)cc1)C(F)C1CC1</smiles>

1-(Biphenyl-4-yl)-2-cyclopropyl-2-fluoroethanone (2z): White solid ; m.p. 65-67 ${ }^{\circ} \mathrm{C} ; 80 \%$ yield (40 mg); ${ }^{1} \mathrm{H}$ NMR $\left(400 \mathrm{MHz}, \mathrm{CDCl}_{3}\right) \delta 8.11(\mathrm{~d}, J=8.1 \mathrm{~Hz}, 2 \mathrm{H})$, $7.72(\mathrm{~d}, J=8.2 \mathrm{~Hz}, 2 \mathrm{H}), 7.64(\mathrm{~d}, J=7.5 \mathrm{~Hz}, 2 \mathrm{H}), 7.49(\mathrm{t}, J=7.4 \mathrm{~Hz}, 2 \mathrm{H}), 7.42(\mathrm{t}, J=$ $7.2 \mathrm{~Hz}, 1 \mathrm{H}), 4.94(\mathrm{dd}, J=49.2,8.3 \mathrm{~Hz}, 1 \mathrm{H}), 1.50-1.36(\mathrm{~m}, 1 \mathrm{H}), 0.84-0.54(\mathrm{~m}, 4 \mathrm{H})$; ${ }^{13} \mathrm{C} \mathrm{NMR}\left(151 \mathrm{MHz}, \mathrm{CDCl}_{3}\right.$, overlapping peaks) $\delta 195.4\left(\mathrm{~d}, J_{C-F}=22.8 \mathrm{~Hz}\right), 146.6$, 139.8, 133.2, $129.9\left(\mathrm{~d}, J_{C-F}=3.8 \mathrm{~Hz}\right), 129.1,128.6,127.5\left(\mathrm{~d}, J_{C-F}=3.9 \mathrm{~Hz}\right), 97.5(\mathrm{~d}$, $\left.J_{C-F}=184.6 \mathrm{~Hz}\right), 13.4\left(\mathrm{~d}, J_{C-F}=26.3 \mathrm{~Hz}\right), 3.8\left(\mathrm{~d}, J_{C-F}=3.1 \mathrm{~Hz}\right), 3.5\left(\mathrm{~d}, J_{C-F}=8.4 \mathrm{~Hz}\right)$; ${ }^{19} \mathrm{~F}$ NMR (564 MHz, $\left.\mathrm{CDCl}_{3}\right) \delta-176.36--187.62$ (m, 1F). HRMS(CI) calcd $\mathrm{C}_{17} \mathrm{H}_{16} \mathrm{FO}[\mathrm{M}+\mathrm{H}]^{+}: 255.1185$, found: 255.1179 .<smiles>CC1(C)CCCC(C)(C)N1OCC(=O)c1ccc(-c2ccccc2)cc1</smiles>

1-(Biphenyl-4-yl)-2-(2,2,6,6-tetramethylpiperidin-1-yloxy)ethanone (3a): Yellow solid; m.p. 132-134 ${ }^{\circ} \mathrm{C} ; 45 \%$ yield $(30 \mathrm{mg}) ;{ }^{1} \mathrm{H}$ NMR $\left(400 \mathrm{MHz}, \mathrm{CDCl}_{3}\right) \delta 8.02(\mathrm{~d}, J$ $=8.1 \mathrm{~Hz}, 2 \mathrm{H}), 7.69(\mathrm{~d}, J=8.1 \mathrm{~Hz}, 2 \mathrm{H}), 7.63(\mathrm{~d}, J=7.5 \mathrm{~Hz}, 2 \mathrm{H}), 7.48(\mathrm{t}, J=7.4 \mathrm{~Hz}$, 2H), $7.40(\mathrm{t}, J=7.2 \mathrm{~Hz}, 1 \mathrm{H}), 5.15(\mathrm{~s}, 2 \mathrm{H}), 1.53-1.44(\mathrm{~m}, 4 \mathrm{H}), 1.40-1.29(\mathrm{~m}, 2 \mathrm{H})$, $1.20(\mathrm{~s}, 12 \mathrm{H}) ;{ }^{13} \mathrm{C}$ NMR $\left(151 \mathrm{MHz}, \mathrm{CDCl}_{3}\right) \delta 195.5,146.1,140.0,134.3,129.1,128.7$, 128.4, 127.4, 127.4, 81.6, 60.3, 39.9, 33.0, 20.4, 17.2; HRMS(CI) calcd $\mathrm{C}_{23} \mathrm{H}_{30} \mathrm{NO}_{2}$ $[\mathrm{M}+\mathrm{H}]^{+}:$352.2277, found: 352.2281 .<smiles>CC1(C)CCCC(C)(C)N1OCC(N)(N)c1ccc(-c2ccccc2)cc1</smiles>

1-(2,2-Diazido-2-(biphenyl-4-yl)ethoxy)-2,2,6,6-tetramethylpiperidine (3b): Yellow solid; m.p. 58-60 ${ }^{\circ} \mathrm{C}$; $15 \%$ yield $(13 \mathrm{mg}) ;{ }^{1} \mathrm{H}$ NMR $\left(400 \mathrm{MHz}, \mathrm{CDCl}_{3}\right) \delta 7.68$ $-7.55(\mathrm{~m}, 6 \mathrm{H}), 7.46$ (t, $J=7.5 \mathrm{~Hz}, 2 \mathrm{H}), 7.37$ (t, $J=7.2 \mathrm{~Hz}, 1 \mathrm{H}), 4.08(\mathrm{~s}, 2 \mathrm{H}), 1.47-$ $1.38(\mathrm{~m}, 4 \mathrm{H}), 1.36-1.22(\mathrm{~m}, 2 \mathrm{H}), 1.13(\mathrm{~s}, 12 \mathrm{H}) ;{ }^{13} \mathrm{C} \mathrm{NMR}\left(151 \mathrm{MHz}, \mathrm{CDCl}_{3}\right) \delta$ $142.3,140.4,135.5,129.0,127.9,127.4,127.3,127.0,85.1,81.9,60.5,40.0,32.9$, 20.3, 17.1; HRMS(CI) calcd $\mathrm{C}_{23} \mathrm{H}_{30} \mathrm{~N}_{7} \mathrm{O}[\mathrm{M}+\mathrm{H}]^{+}$: 420.2512 , found: 420.2510 . 
8. NMR Spectra for the substrates and products

${ }^{1} \mathrm{H}$ NMR of 1a

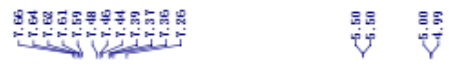<smiles>C=C(N)c1ccc(-c2ccccc2)cc1</smiles>

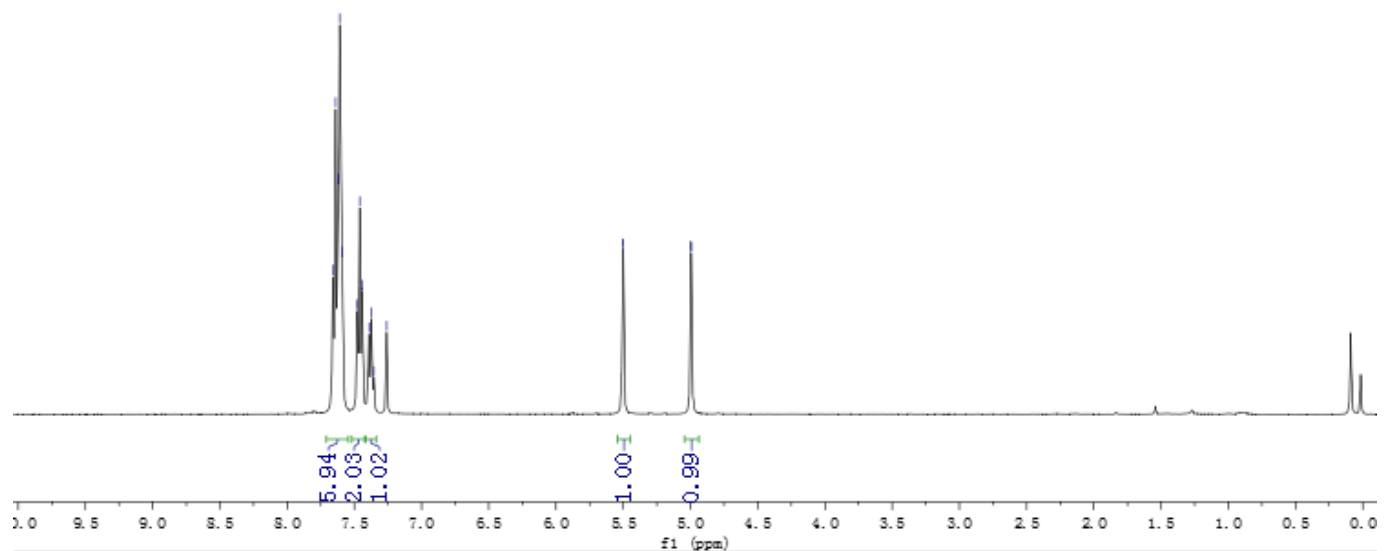

${ }^{13} \mathrm{C}$ NMR of $1 \mathrm{a}$

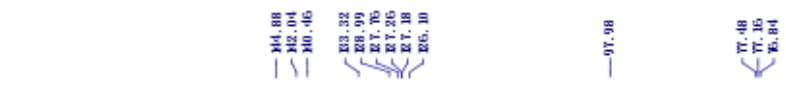<smiles>C=C(N)c1ccc(-c2ccccc2)cc1</smiles>

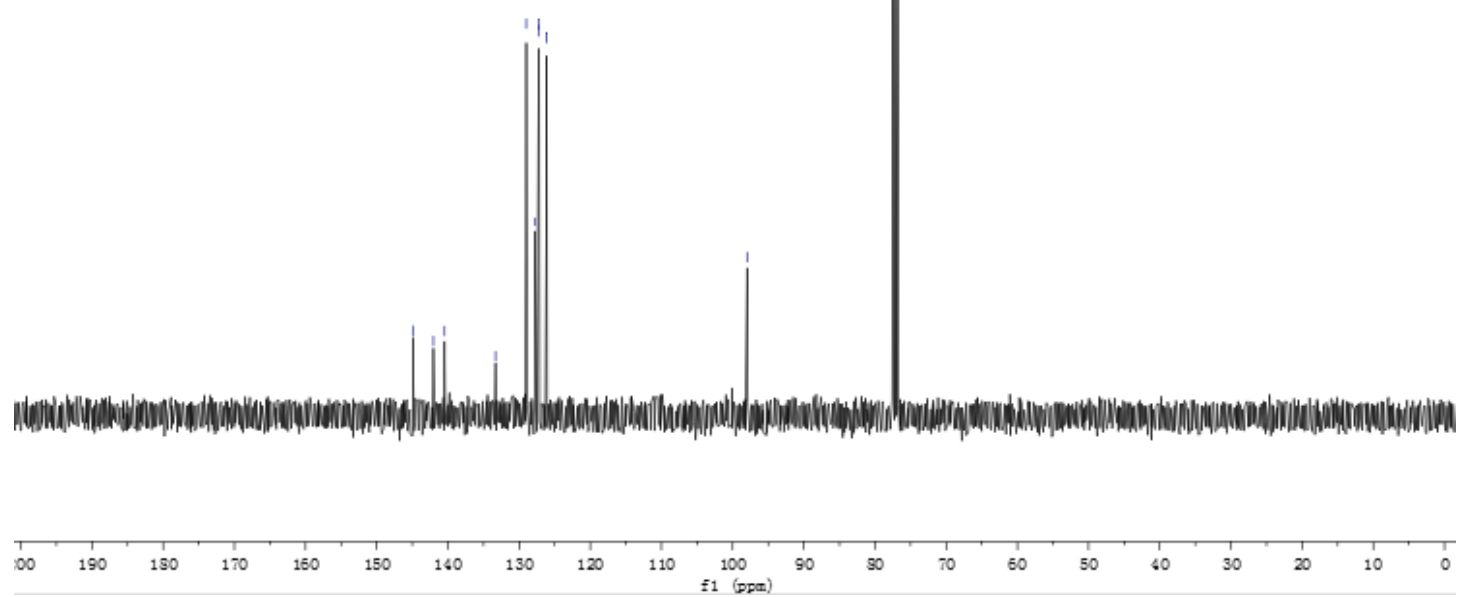


${ }^{1} \mathrm{H}$ NMR of $\mathbf{1 b}$

8

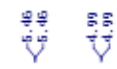

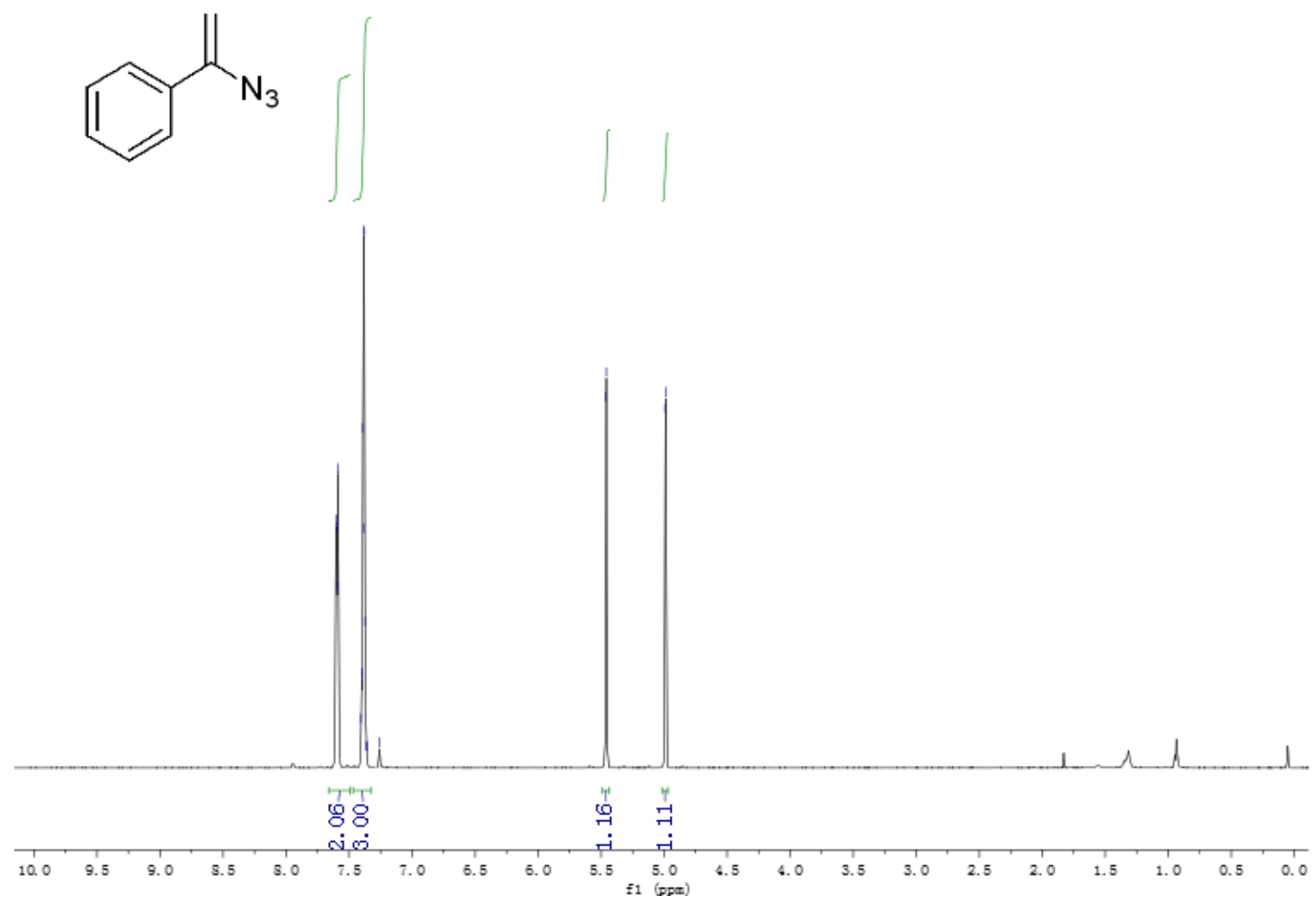

${ }^{13} \mathrm{C}$ NMR of $\mathbf{1 b}$

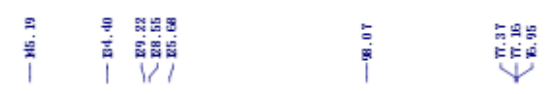<smiles>C=C([N])c1ccccc1</smiles>

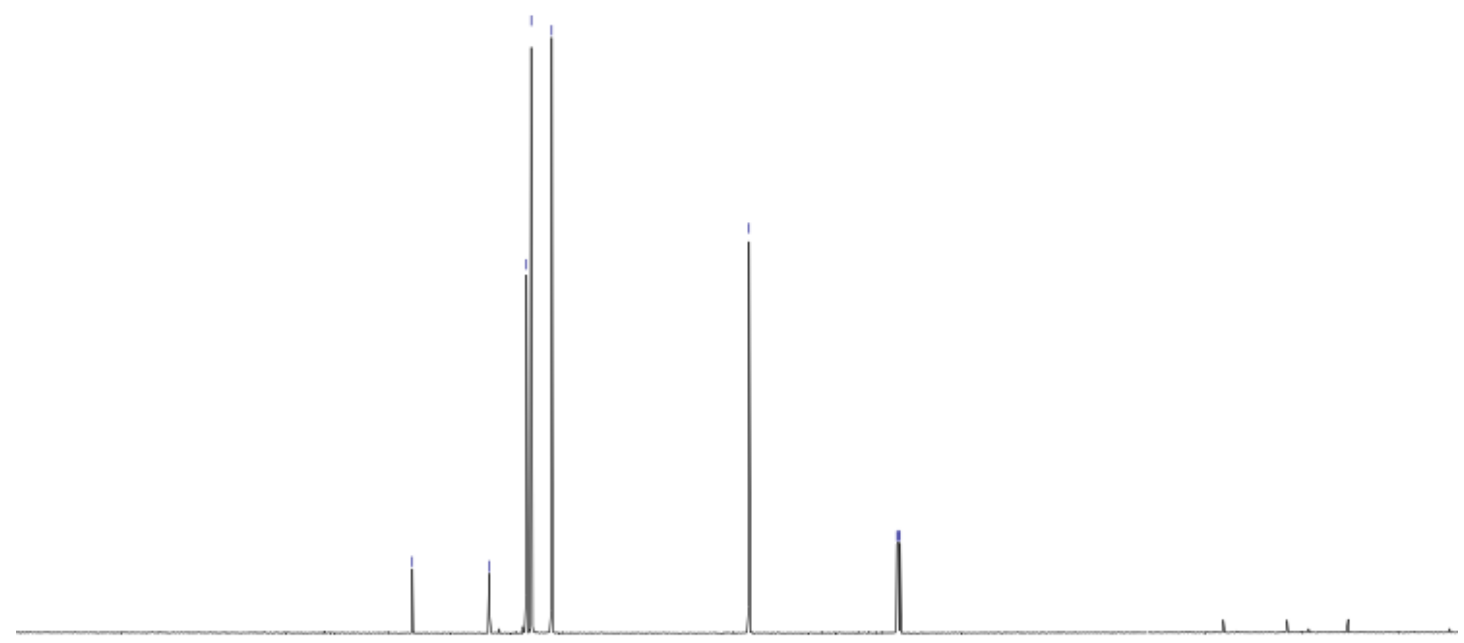

\begin{tabular}{lllllllllllllllllllllll}
\hline 0 & 190 & 130 & 170 & 160 & 150 & 140 & 130 & 120 & 110 & 100 & 90 & 50 & 70 & 60 & 50 & 40 & 30 & 20 & 10 & 0
\end{tabular} 
${ }^{1} \mathrm{H}$ NMR of $\mathbf{1 c}$<smiles>C=C(N)c1ccc(F)cc1</smiles>

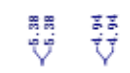

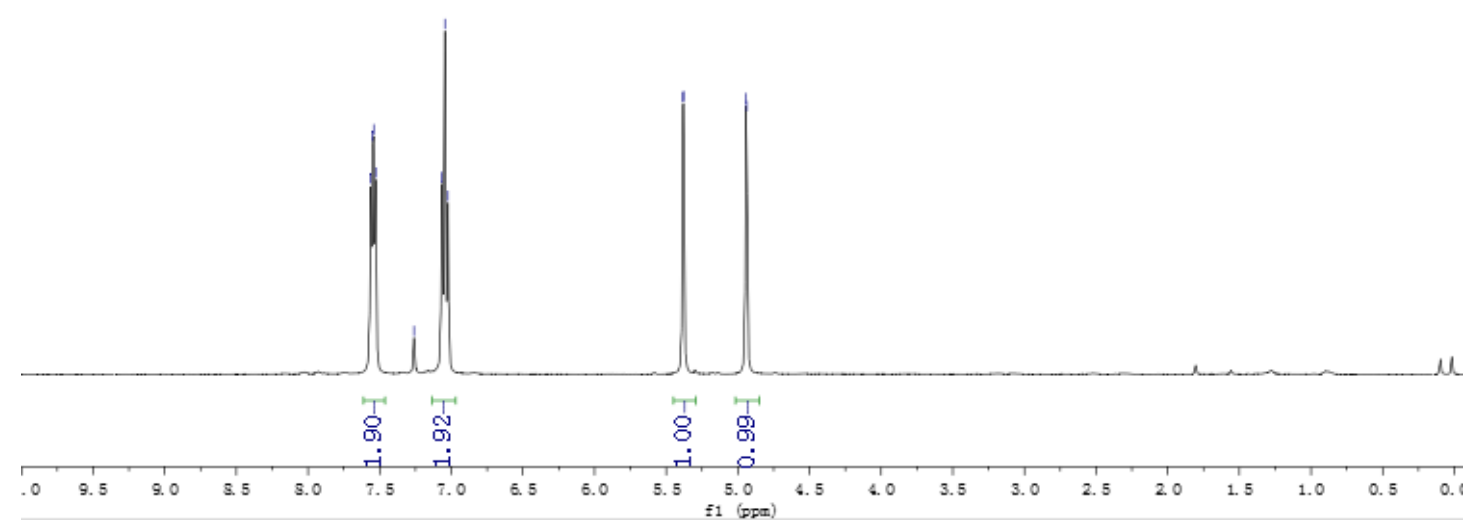

${ }^{13} \mathrm{C}$ NMR of 1c<smiles>NC(=[W])c1ccc(F)cc1</smiles>

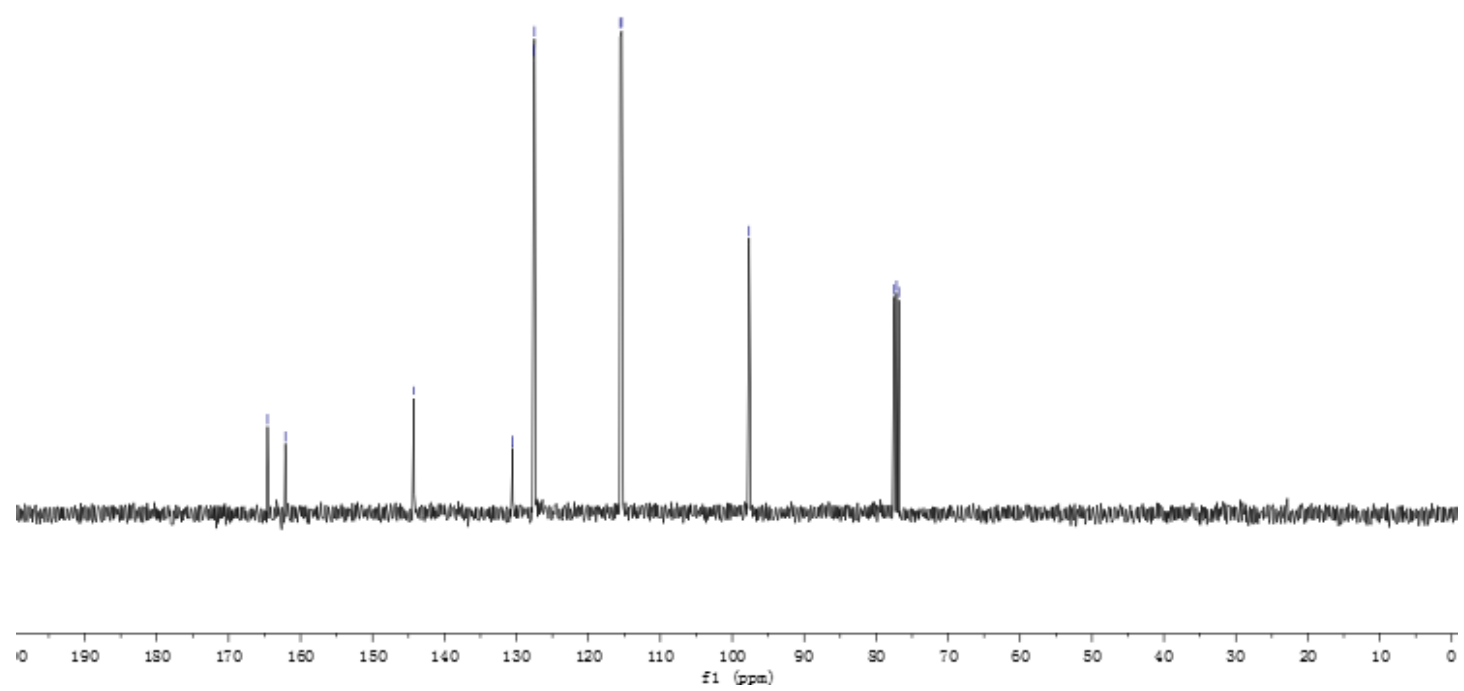


${ }^{1} \mathrm{H}$ NMR of $\mathbf{1 d}$
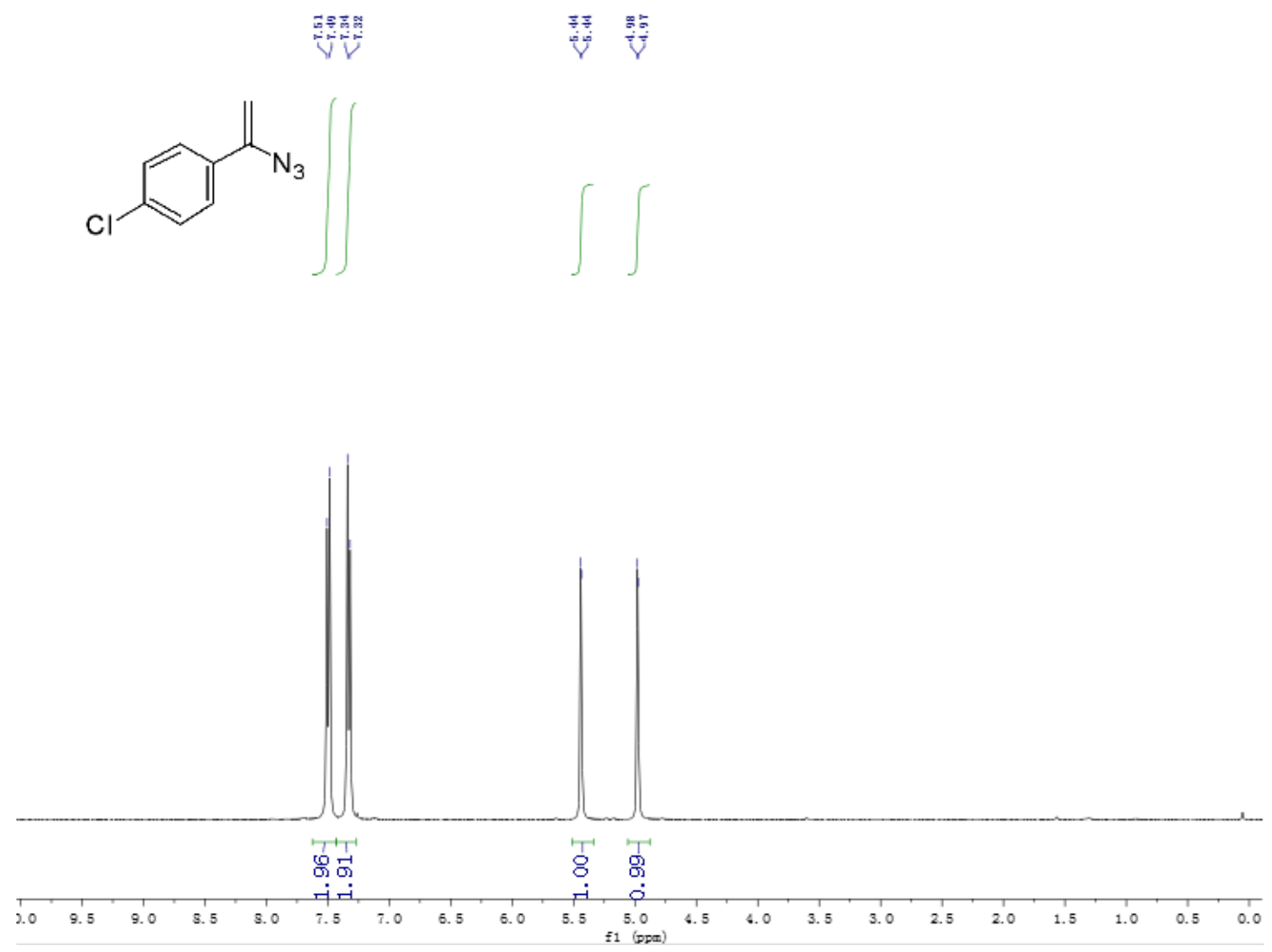

${ }^{13} \mathrm{C}$ NMR of 1d

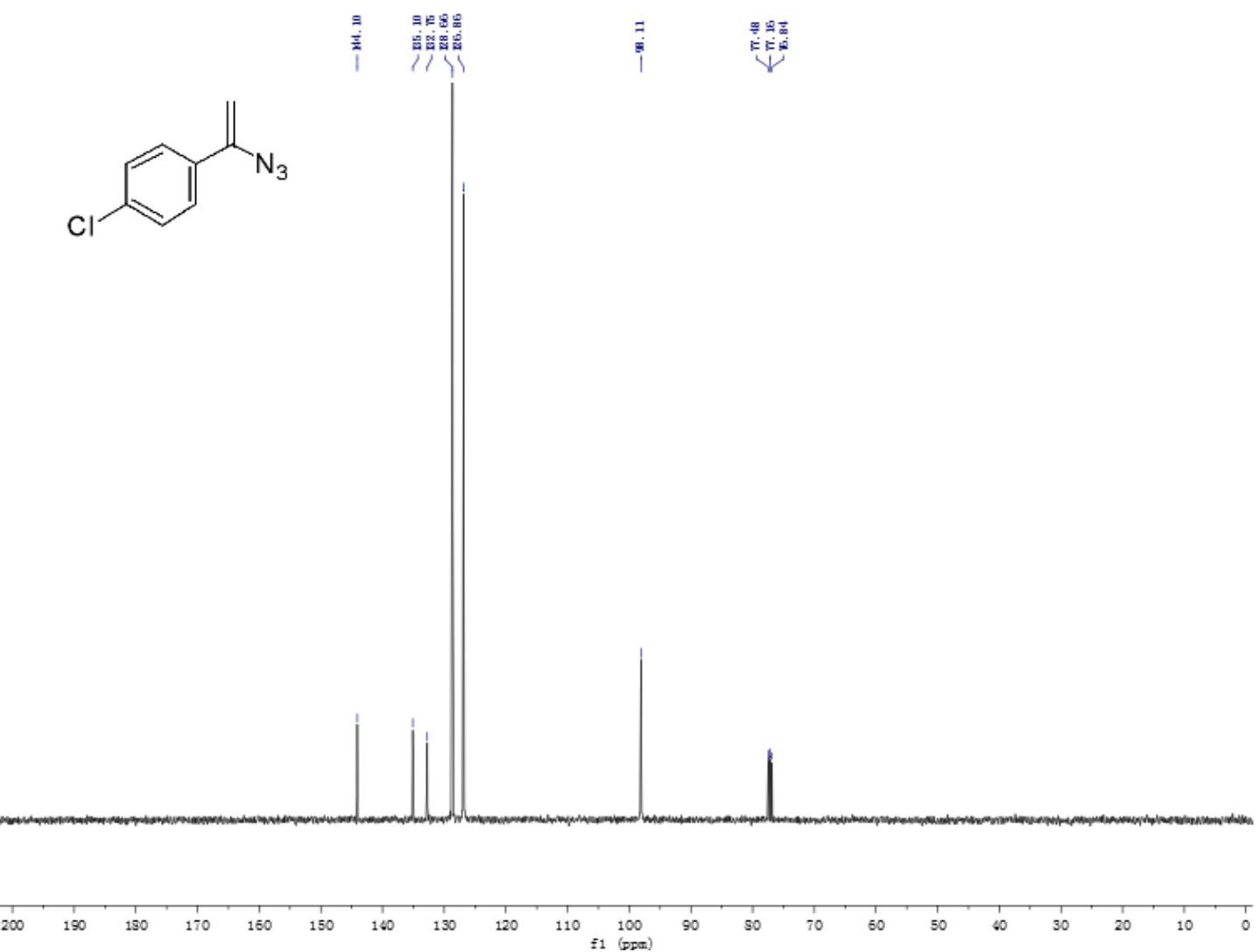


${ }^{1} \mathrm{H}$ NMR of $1 \mathbf{e}$

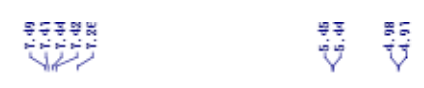
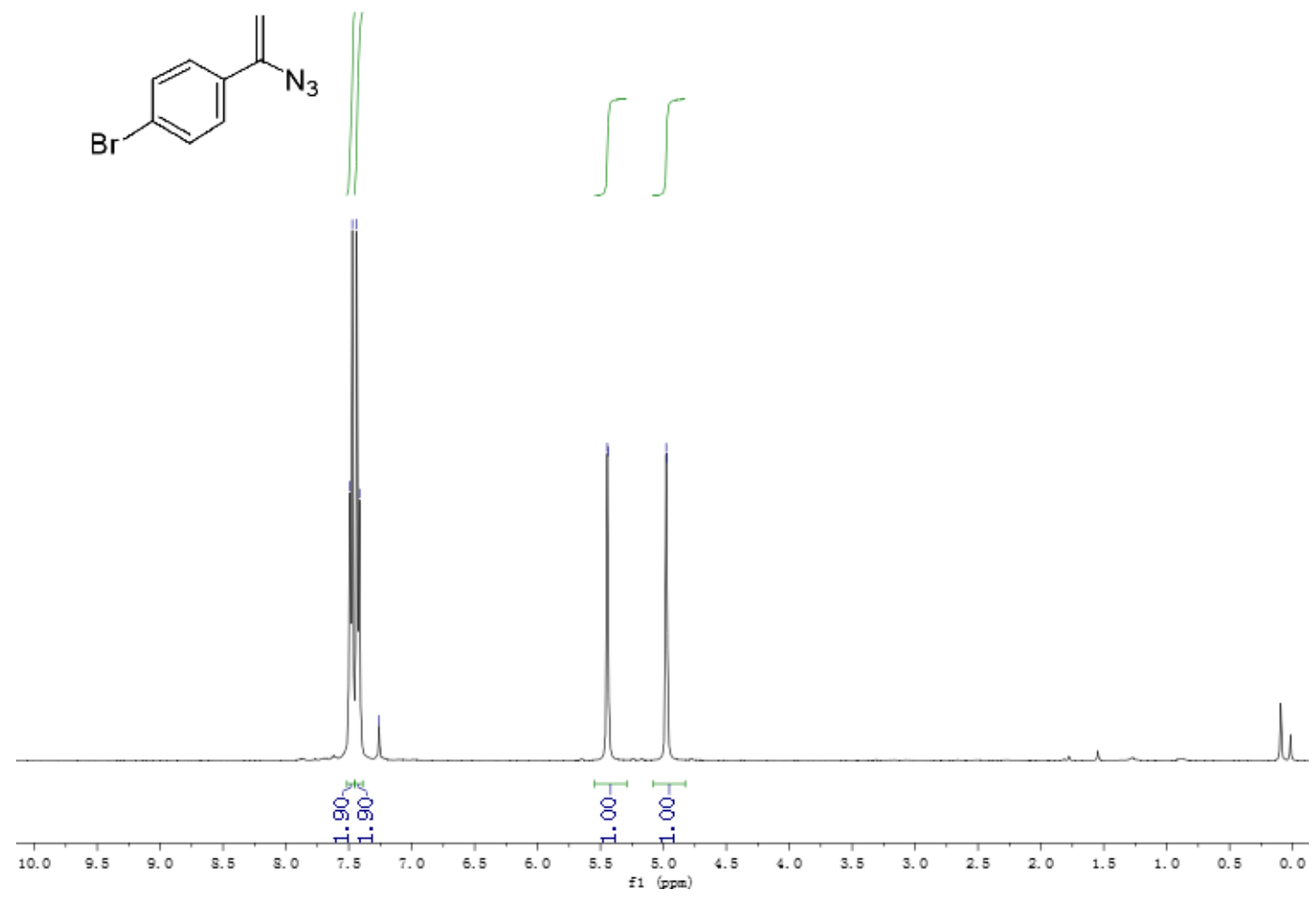

${ }^{13} \mathrm{C}$ NMR of $1 \mathrm{e}$<smiles>C=C(N)c1ccc(Br)cc1</smiles>

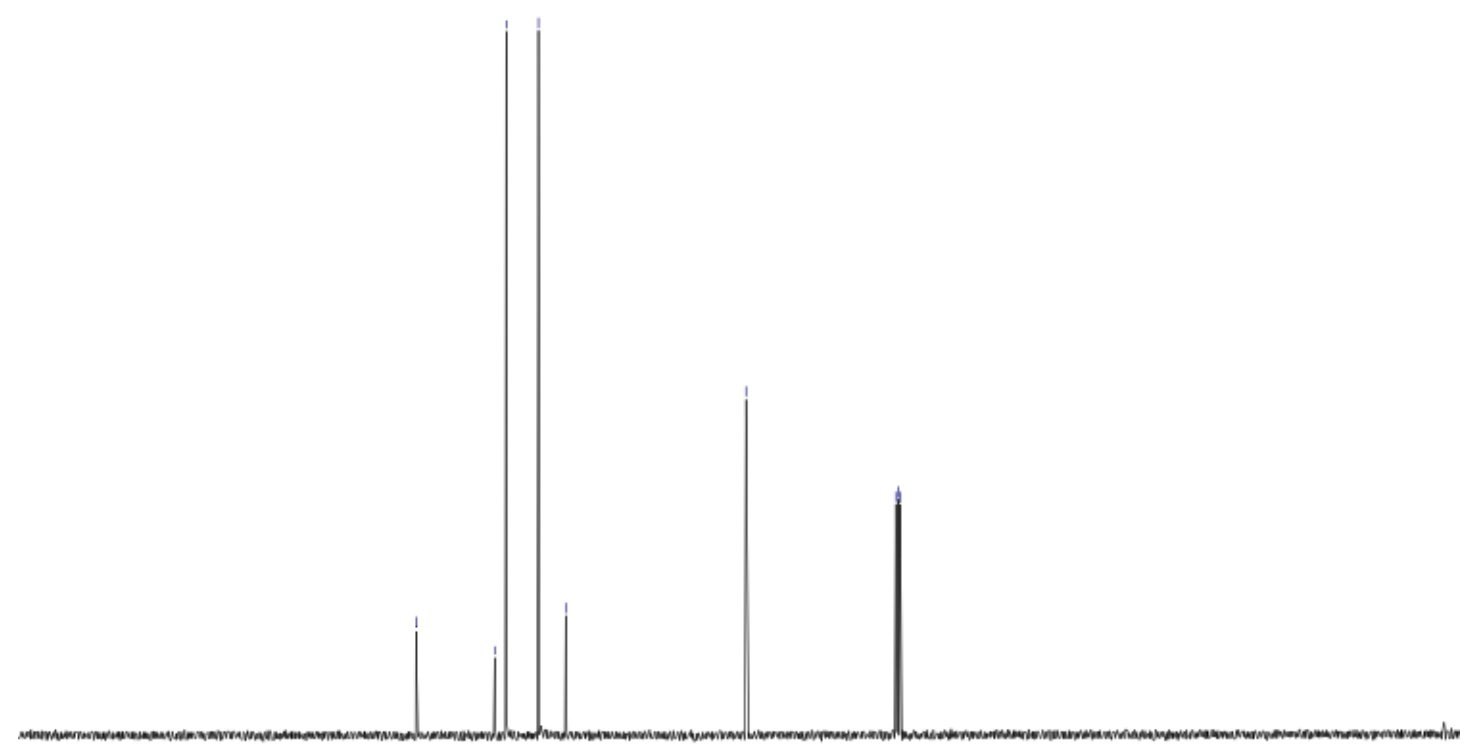

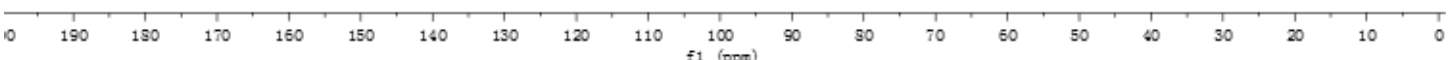


${ }^{1} \mathrm{H}$ NMR of $\mathbf{1 f}$

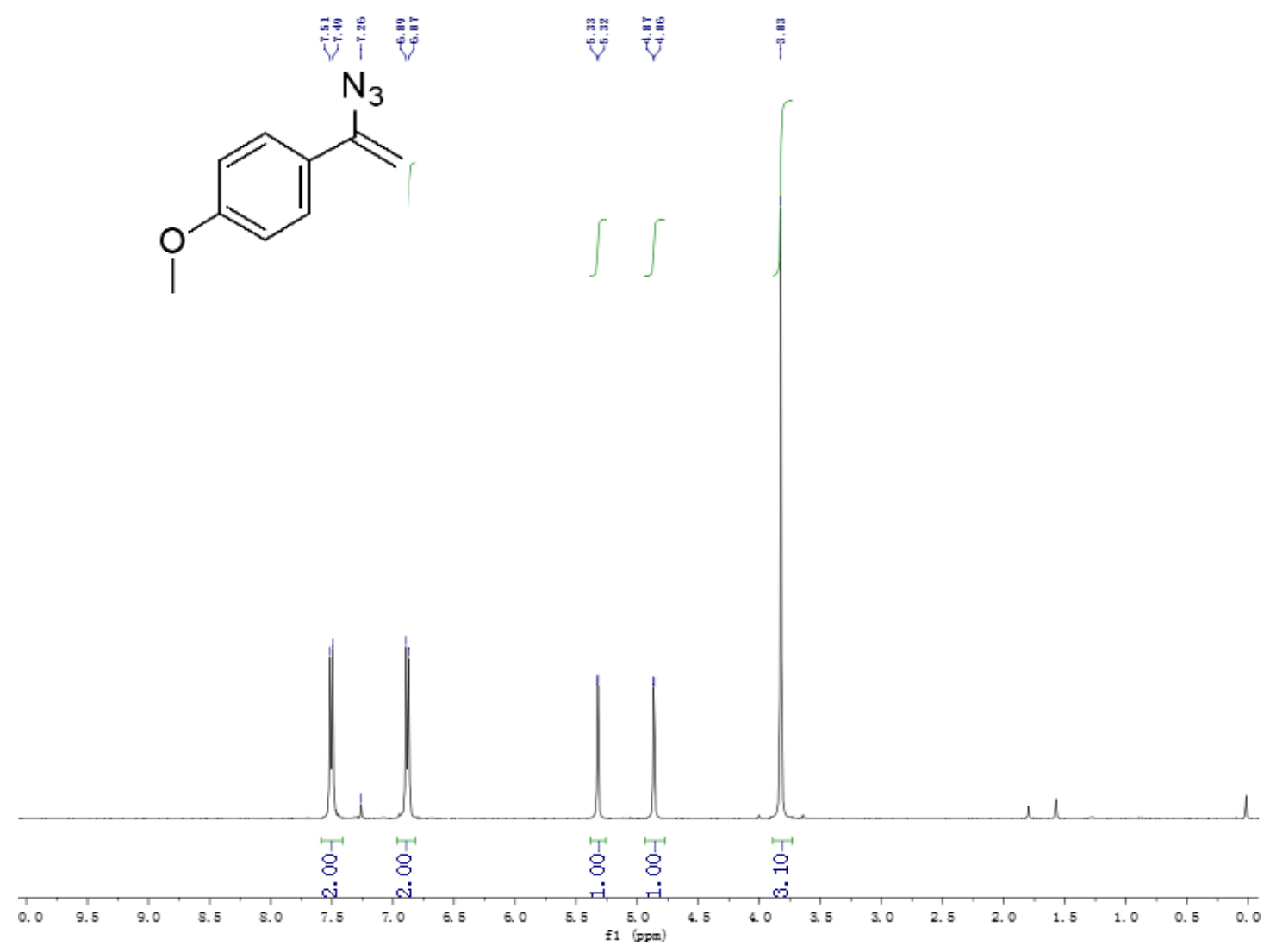

${ }^{13} \mathrm{C}$ NMR of $\mathbf{1 f}$
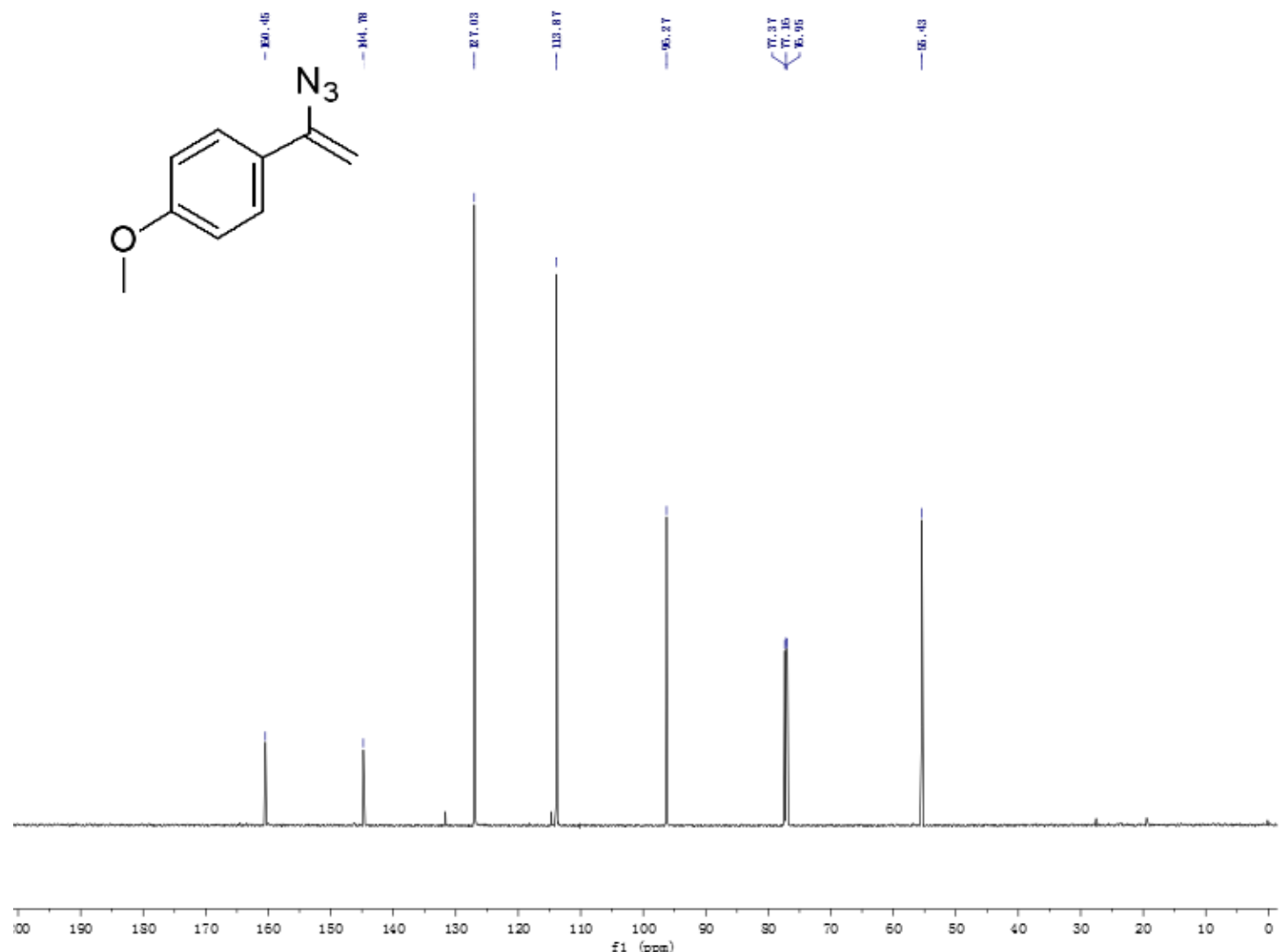
${ }^{1} \mathrm{H}$ NMR of $\mathbf{1 g}$

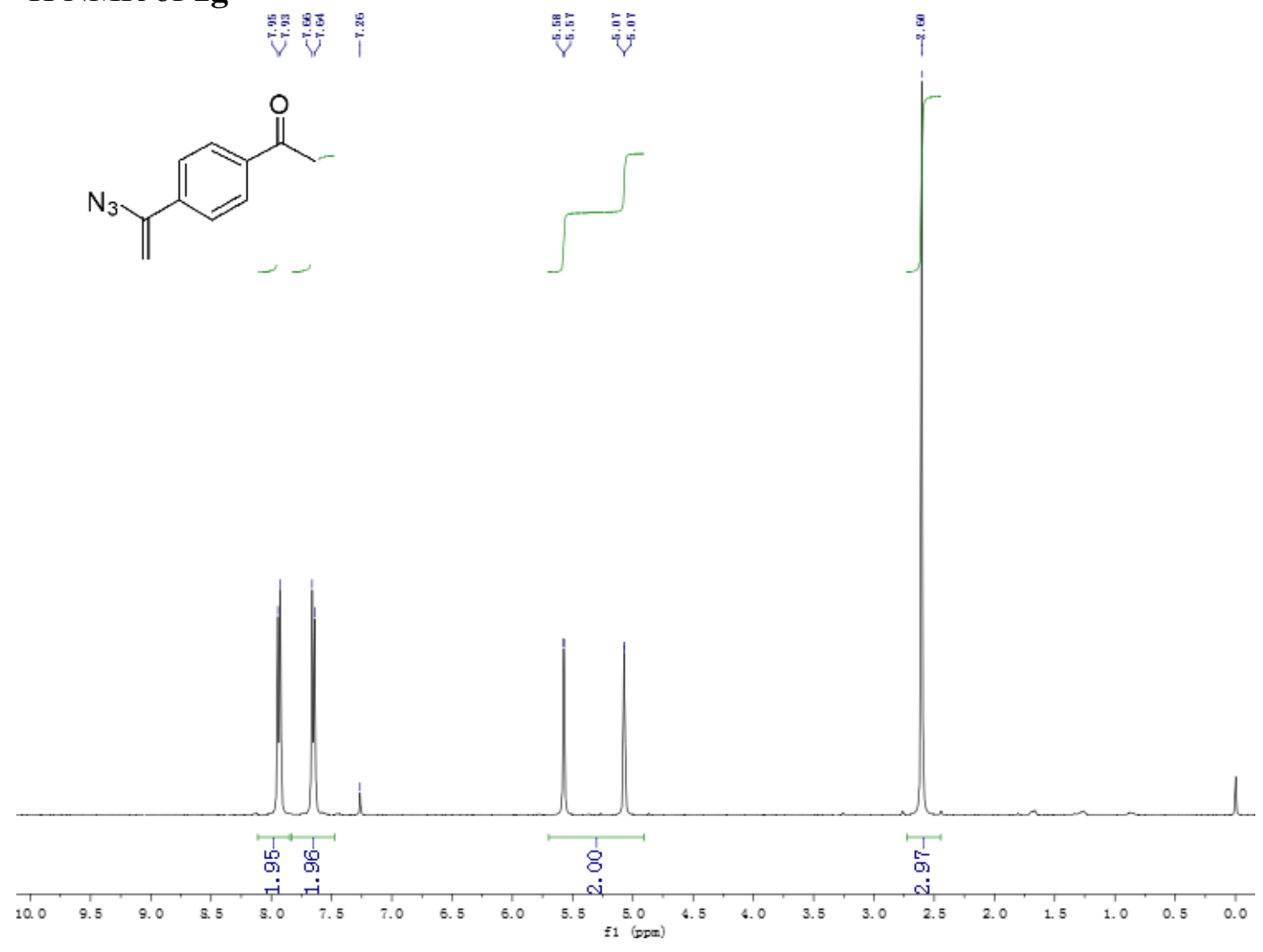

\section{${ }^{13} \mathrm{C}$ NMR of $\mathbf{1 g}$}<smiles>C1CCCCC1</smiles>
i
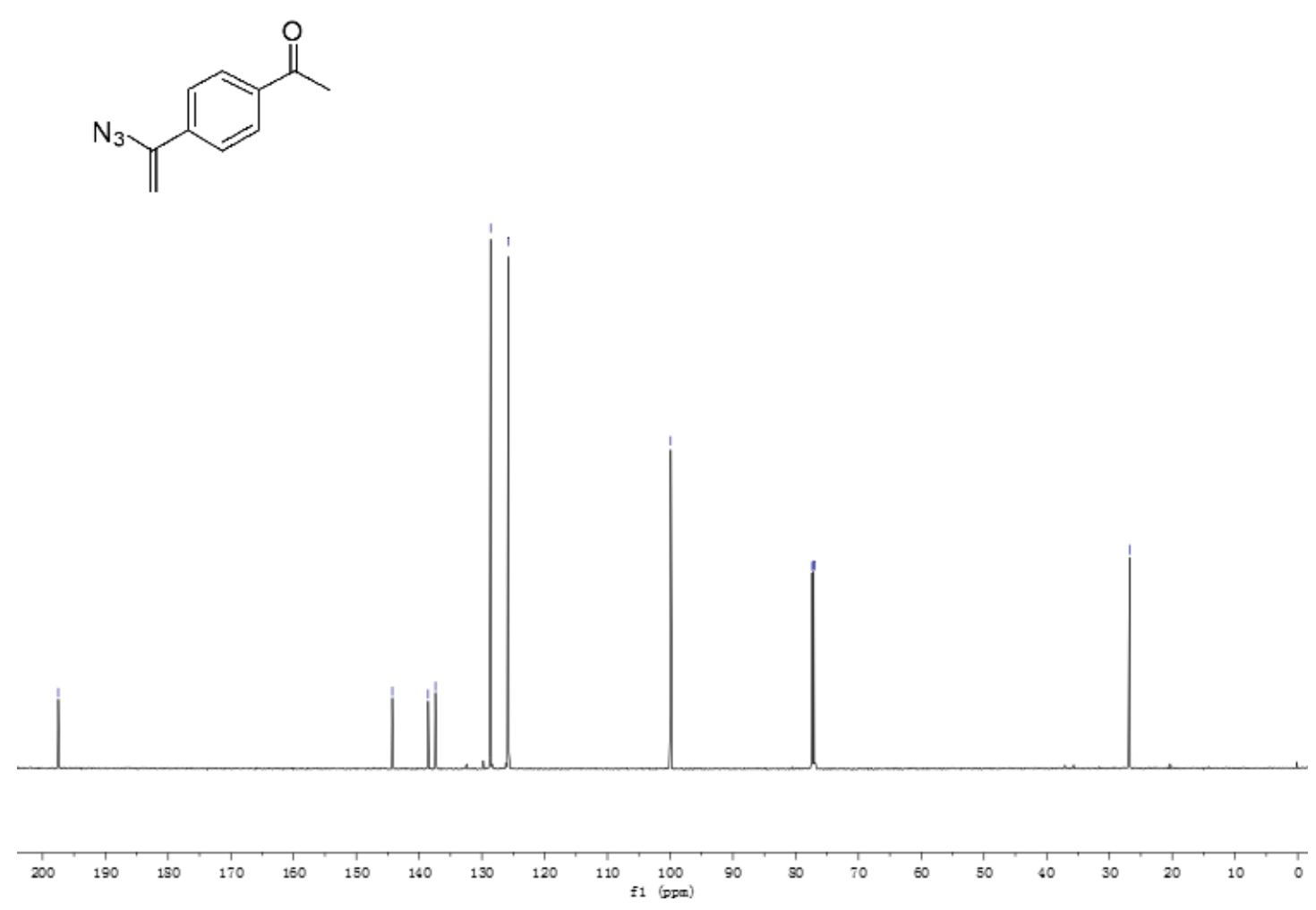
${ }^{1} \mathrm{H}$ NMR of $\mathbf{1 i}$

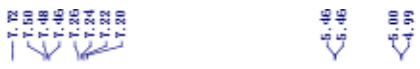<smiles>C=C(N)c1cccc(Br)c1</smiles>

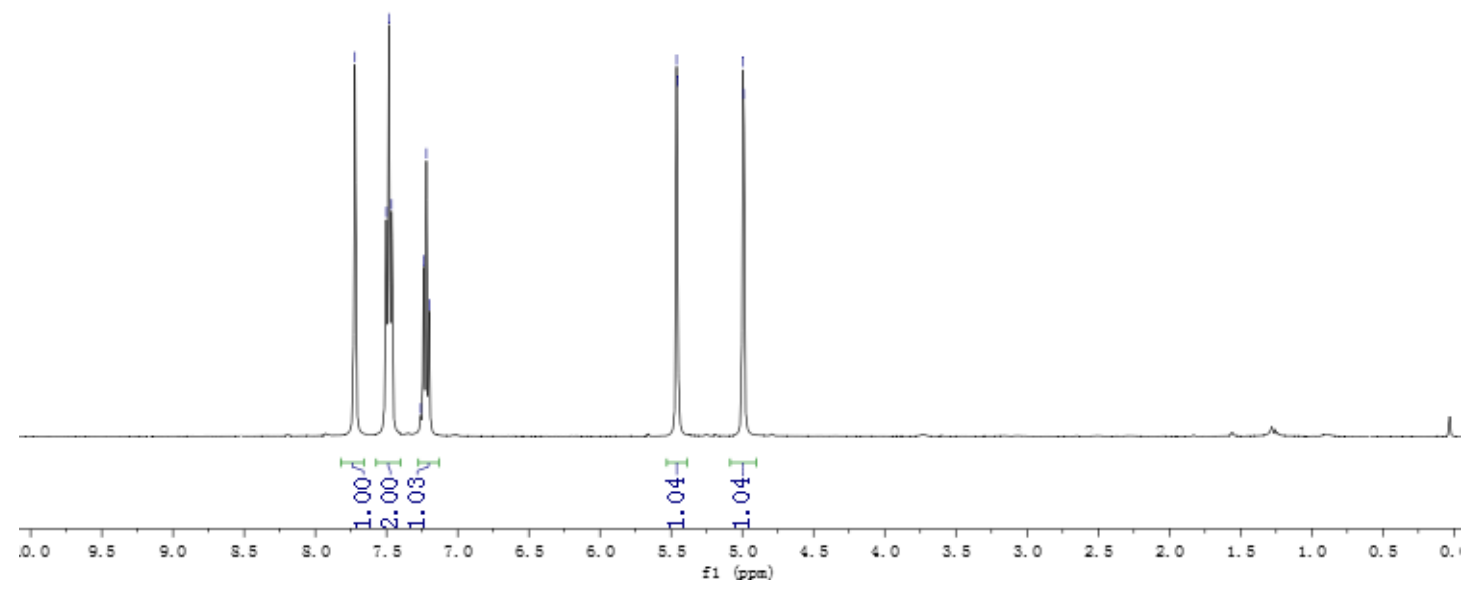

${ }^{13} \mathrm{C}$ NMR of $\mathbf{1 i}$

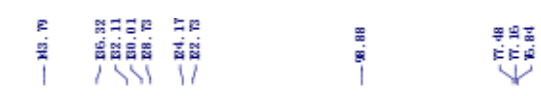
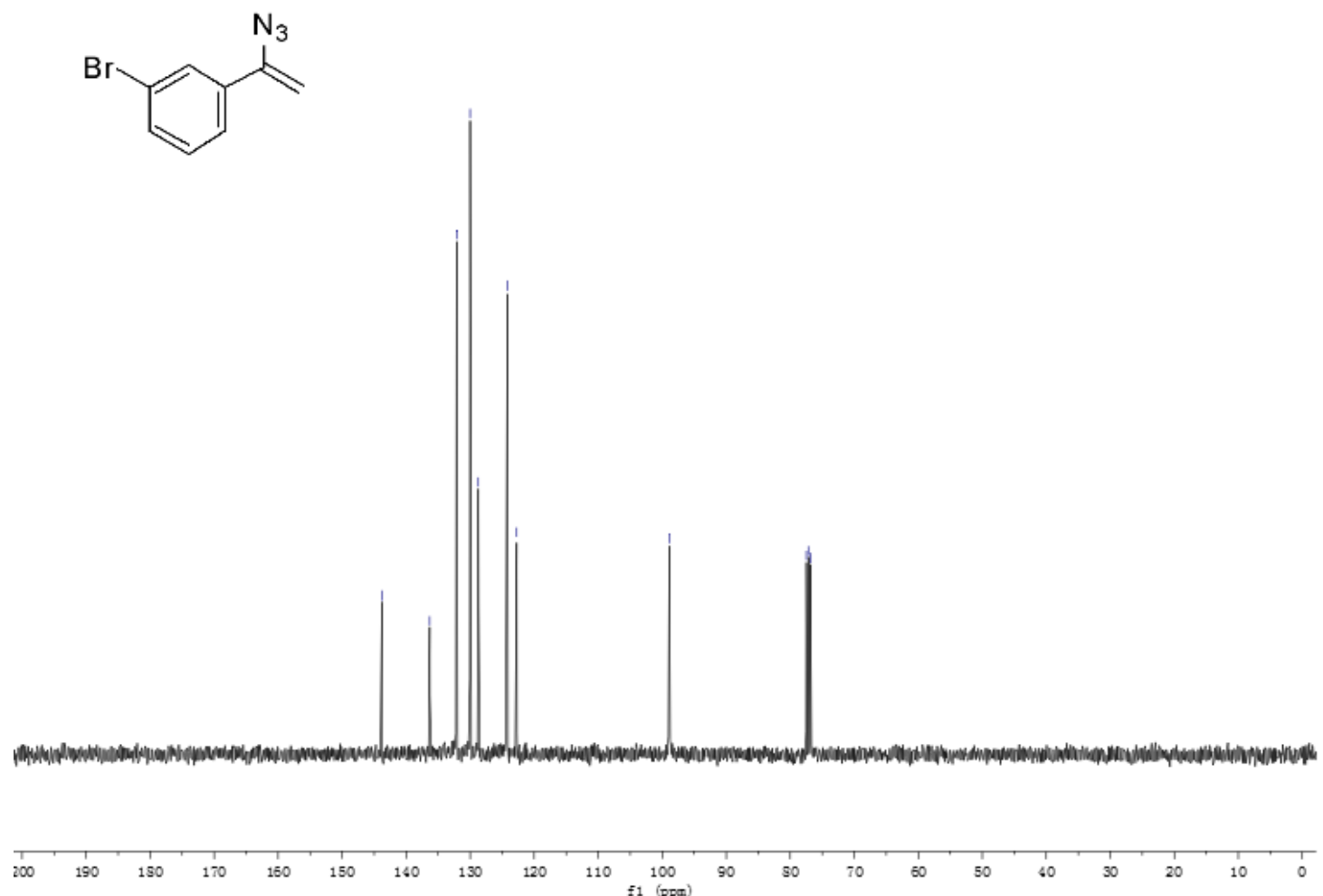
${ }^{1} \mathrm{H}$ NMR of $\mathbf{1 j}$
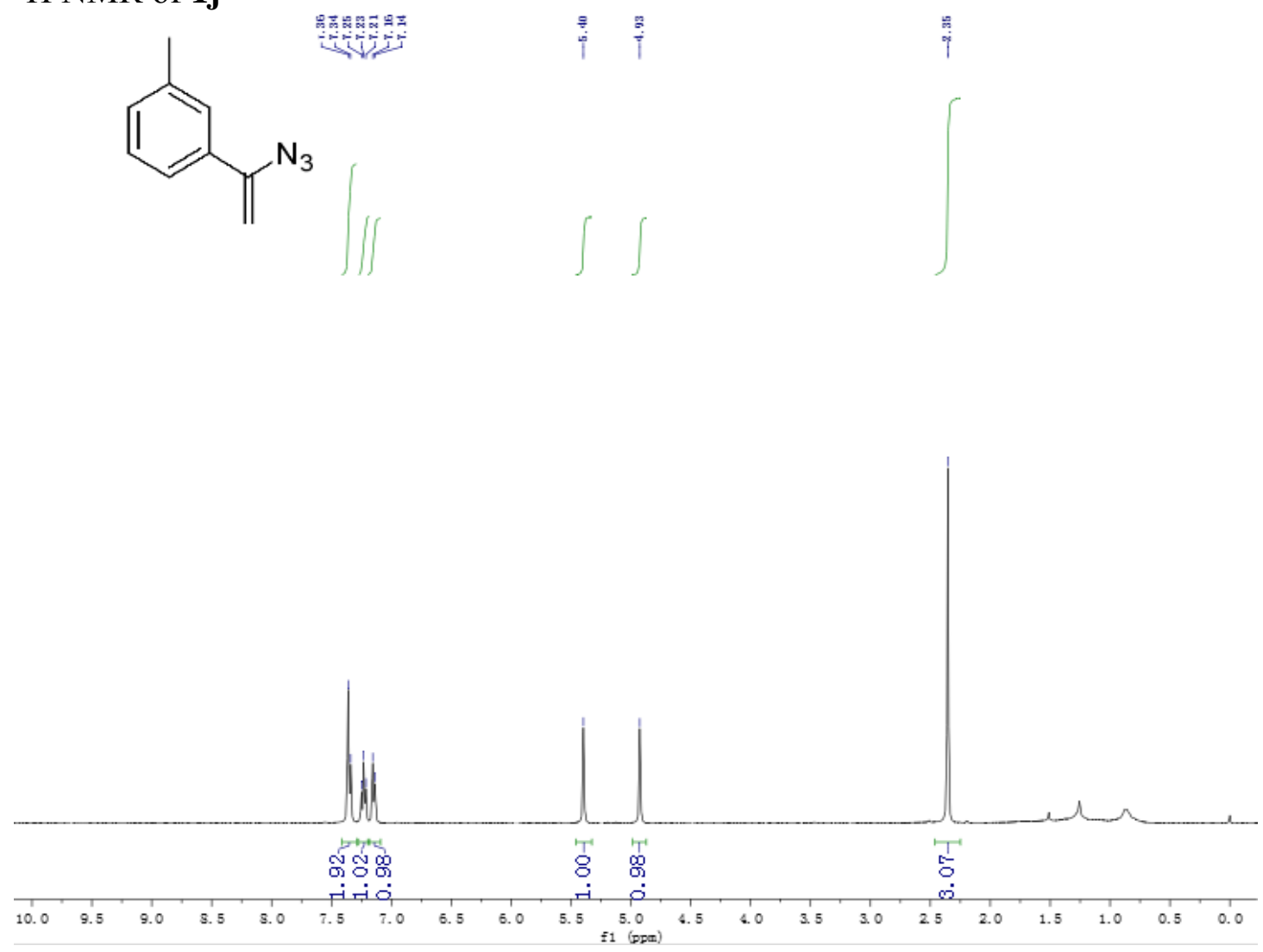

${ }^{13} \mathrm{C}$ NMR of $\mathbf{1 j}$
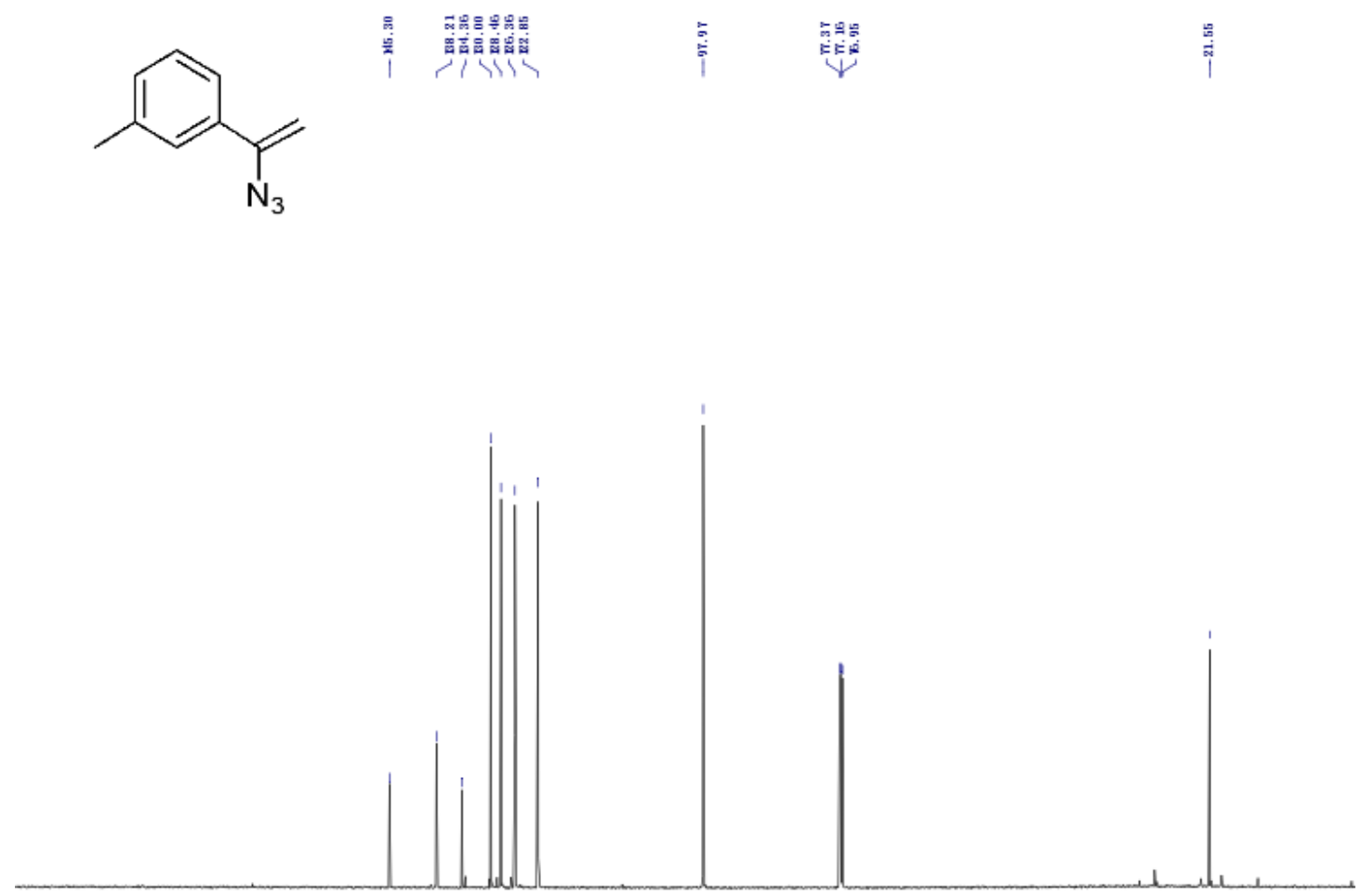

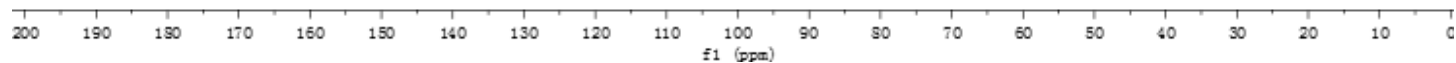


${ }^{1} \mathrm{H}$ NMR of $\mathbf{1 k}$

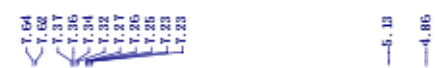
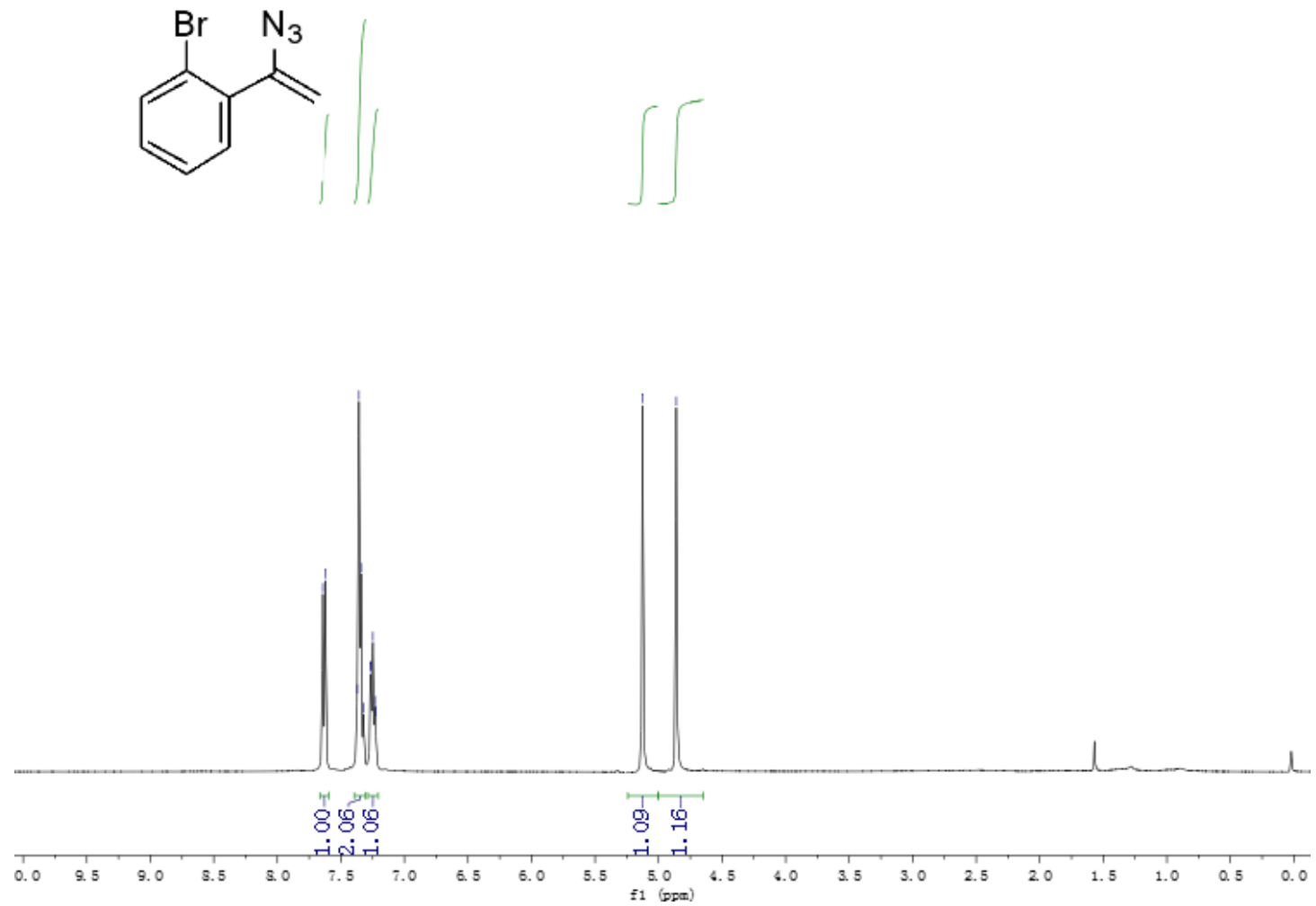

${ }^{13} \mathrm{C}$ NMR of $\mathbf{1 k}$
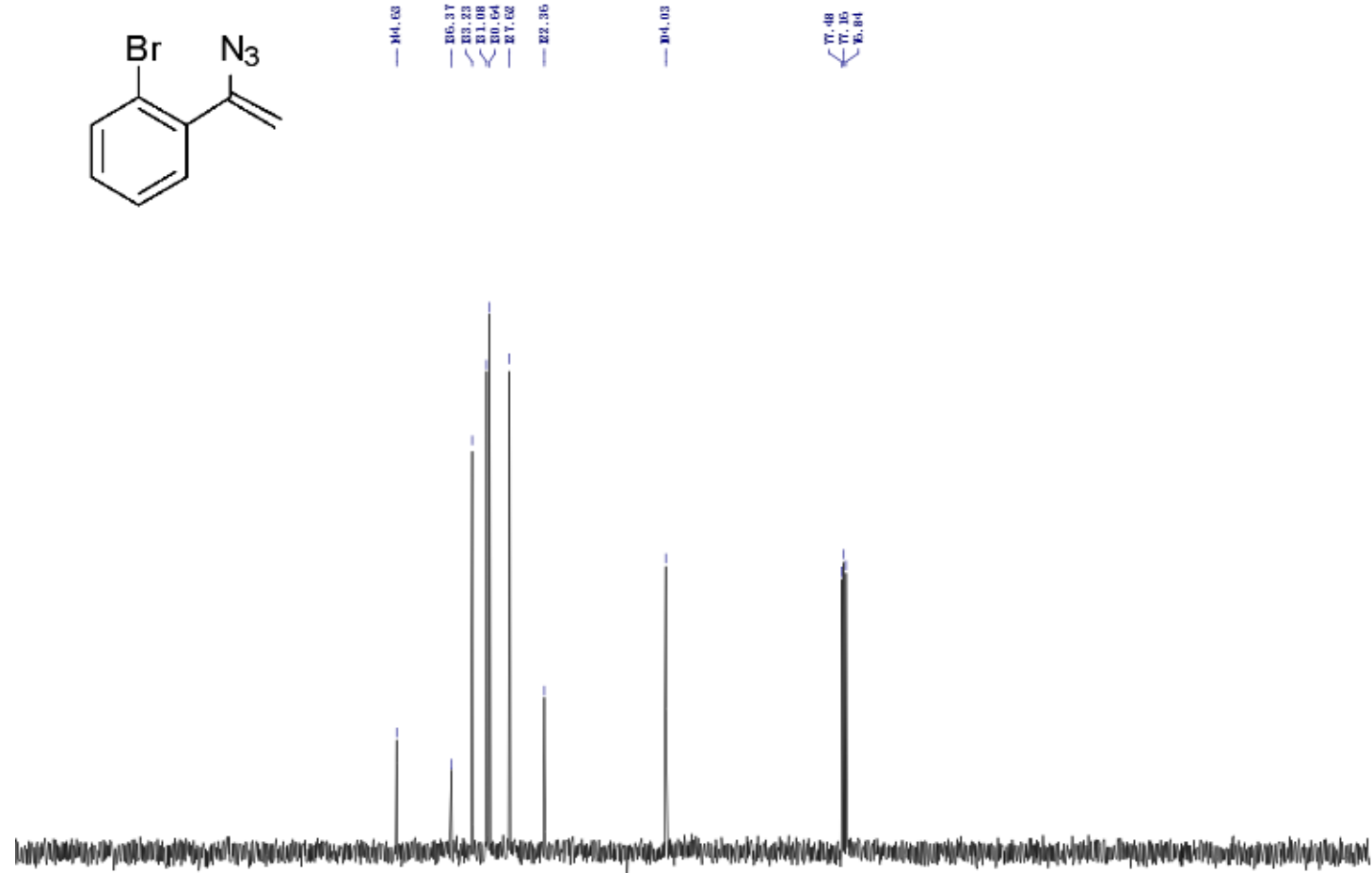

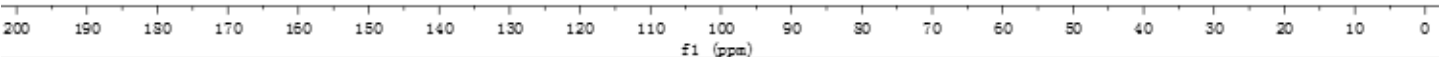


${ }^{1} \mathrm{H}$ NMR of $\mathbf{1 l}$

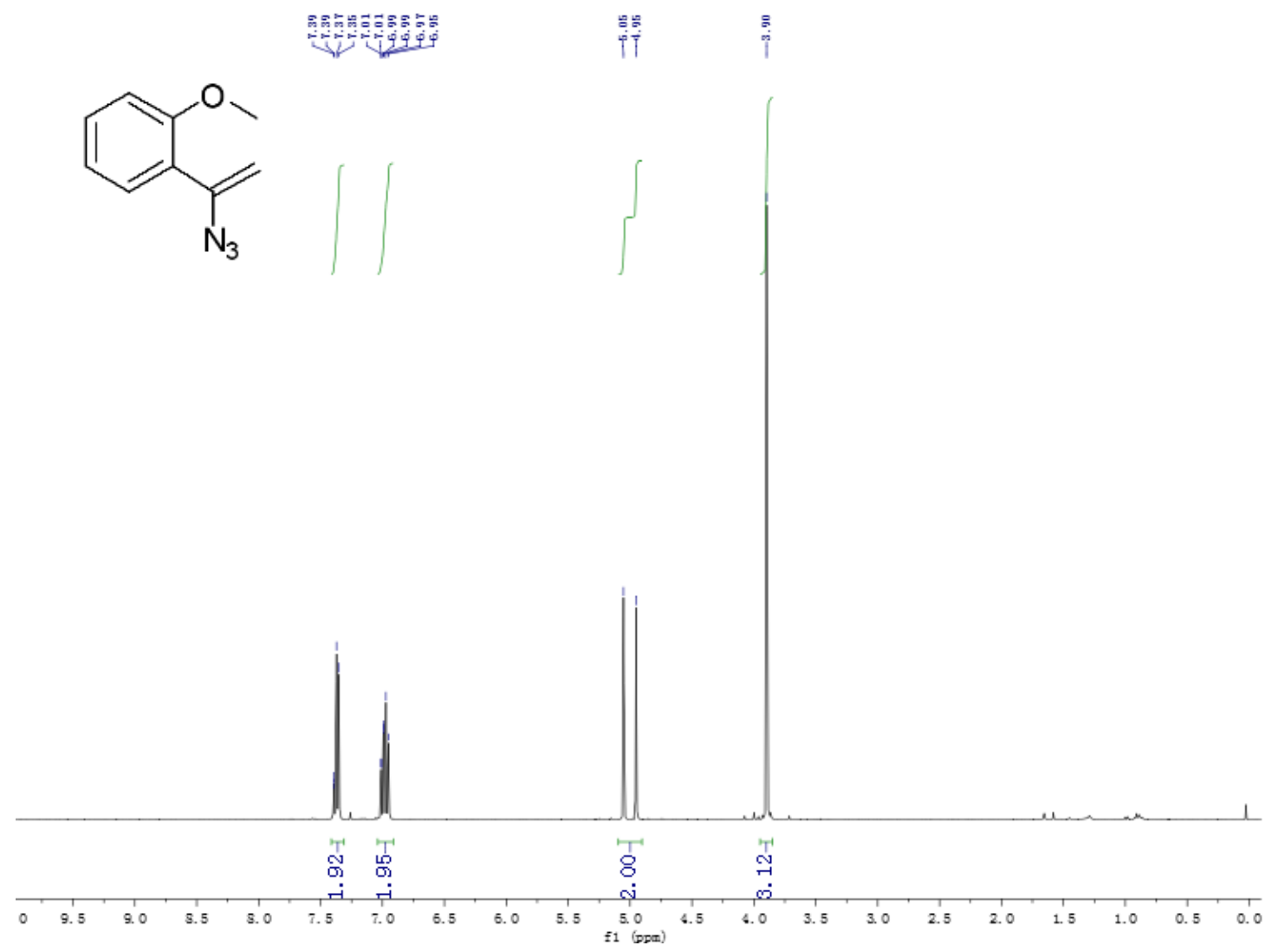

${ }^{13} \mathrm{C}$ NMR of $\mathbf{1 l}$

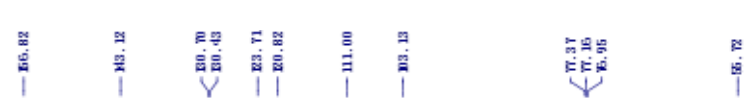<smiles>C=C([N])c1ccccc1OC</smiles>

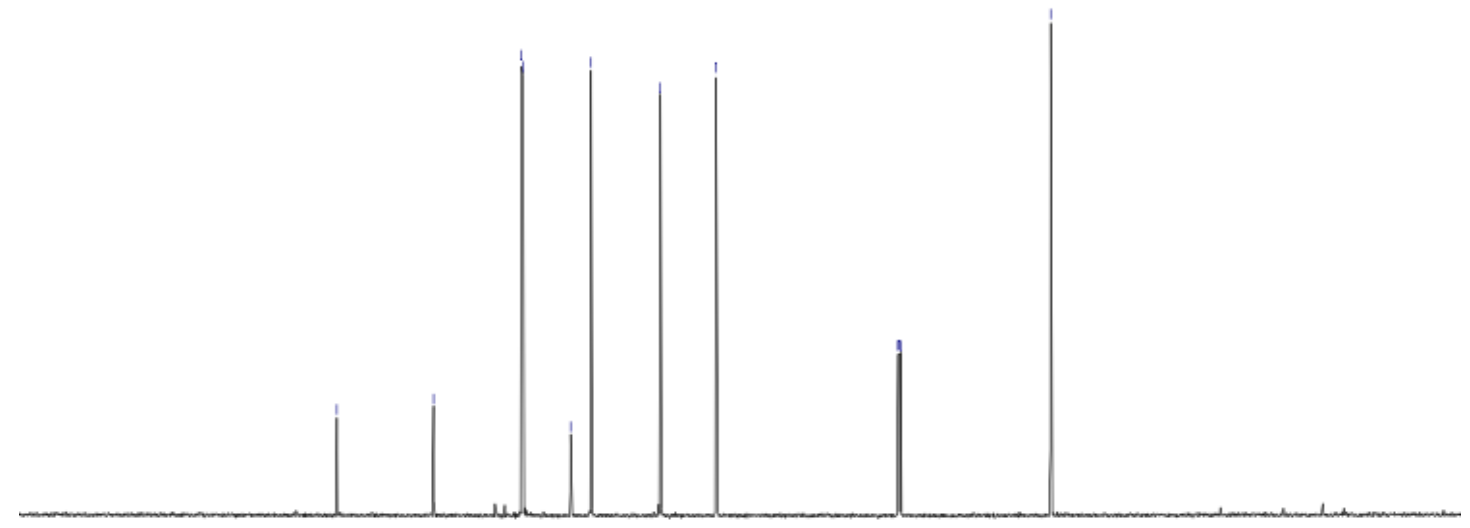

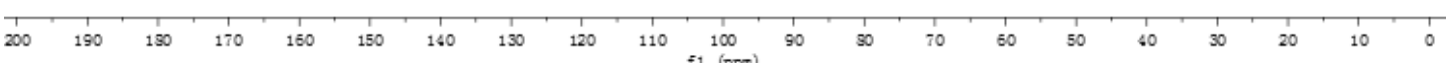


${ }^{1} \mathrm{H}$ NMR of $\mathbf{1 m}$

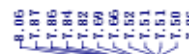

章<smiles>C=C(N)c1ccc2ccccc2c1</smiles>

J) / )

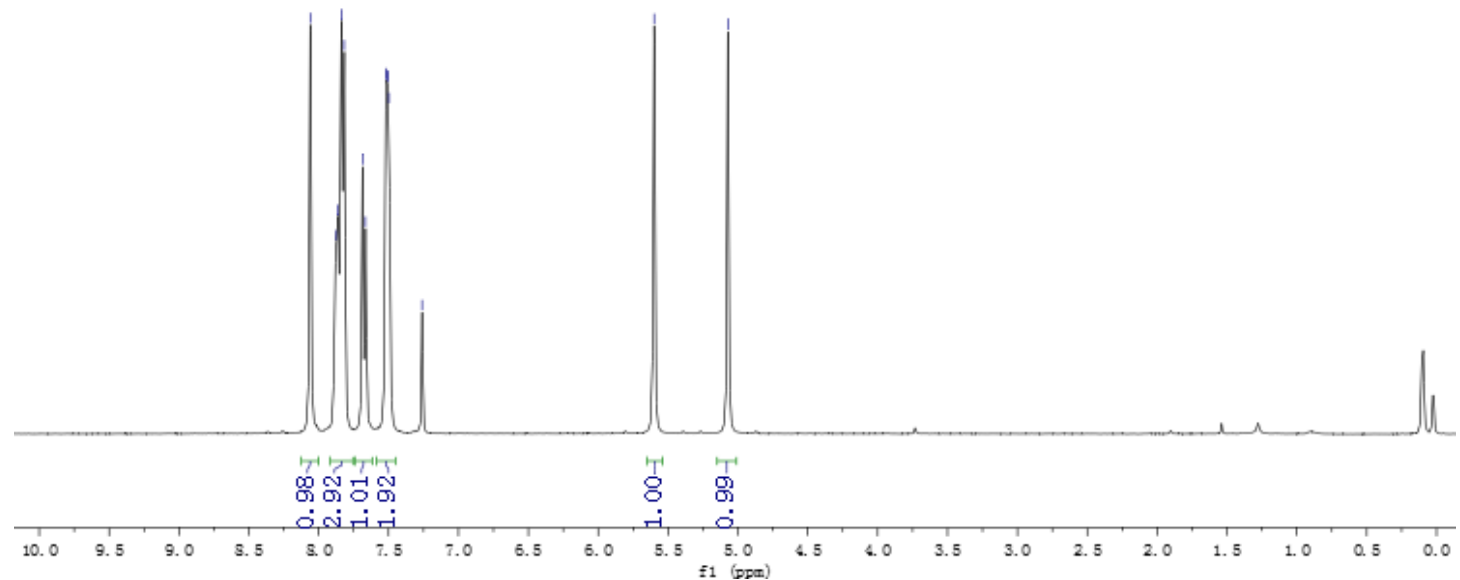

${ }^{13} \mathrm{C}$ NMR of $\mathbf{1 m}$
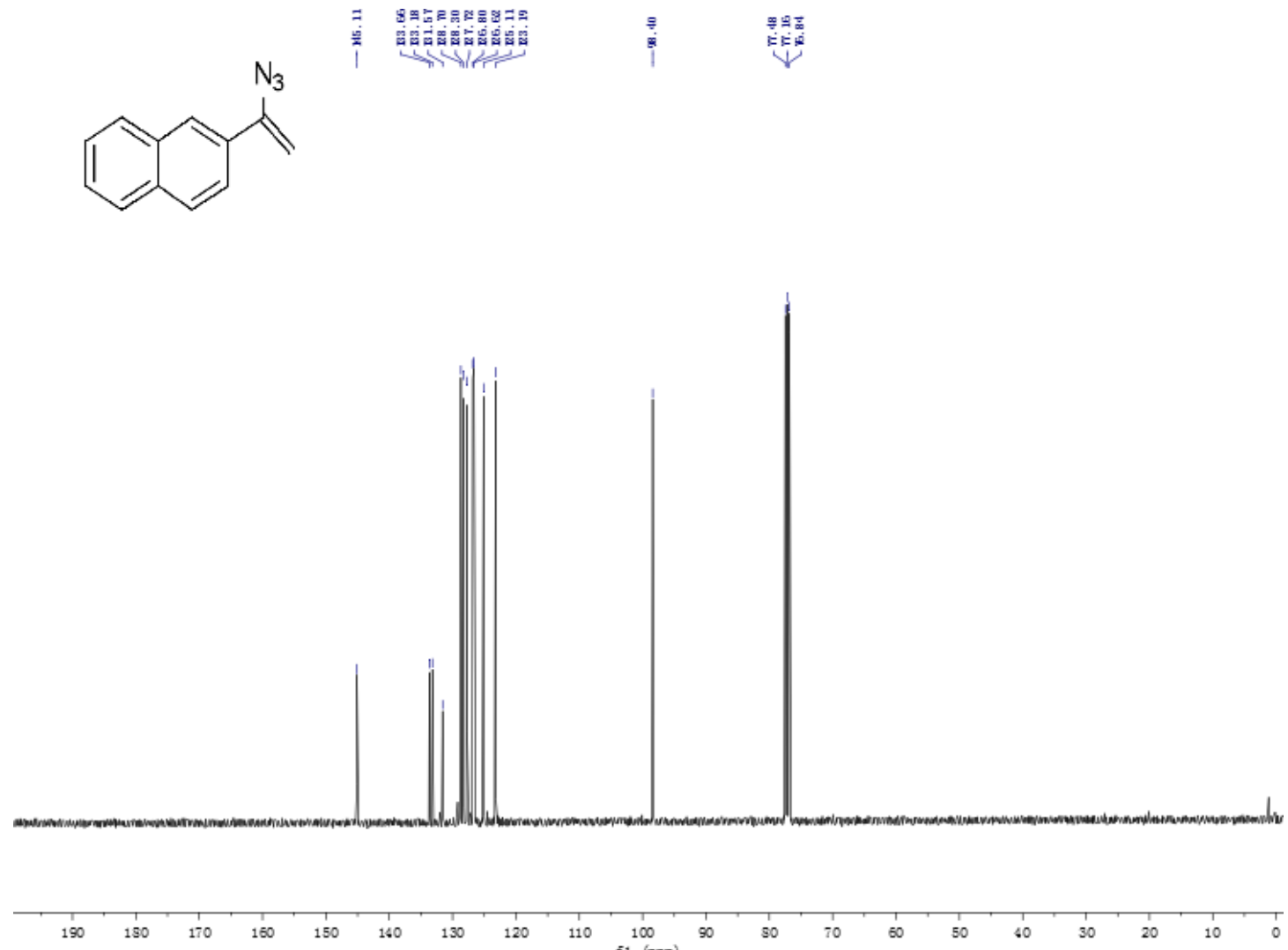
${ }^{1} \mathrm{H}$ NMR of $\mathbf{1 n}$

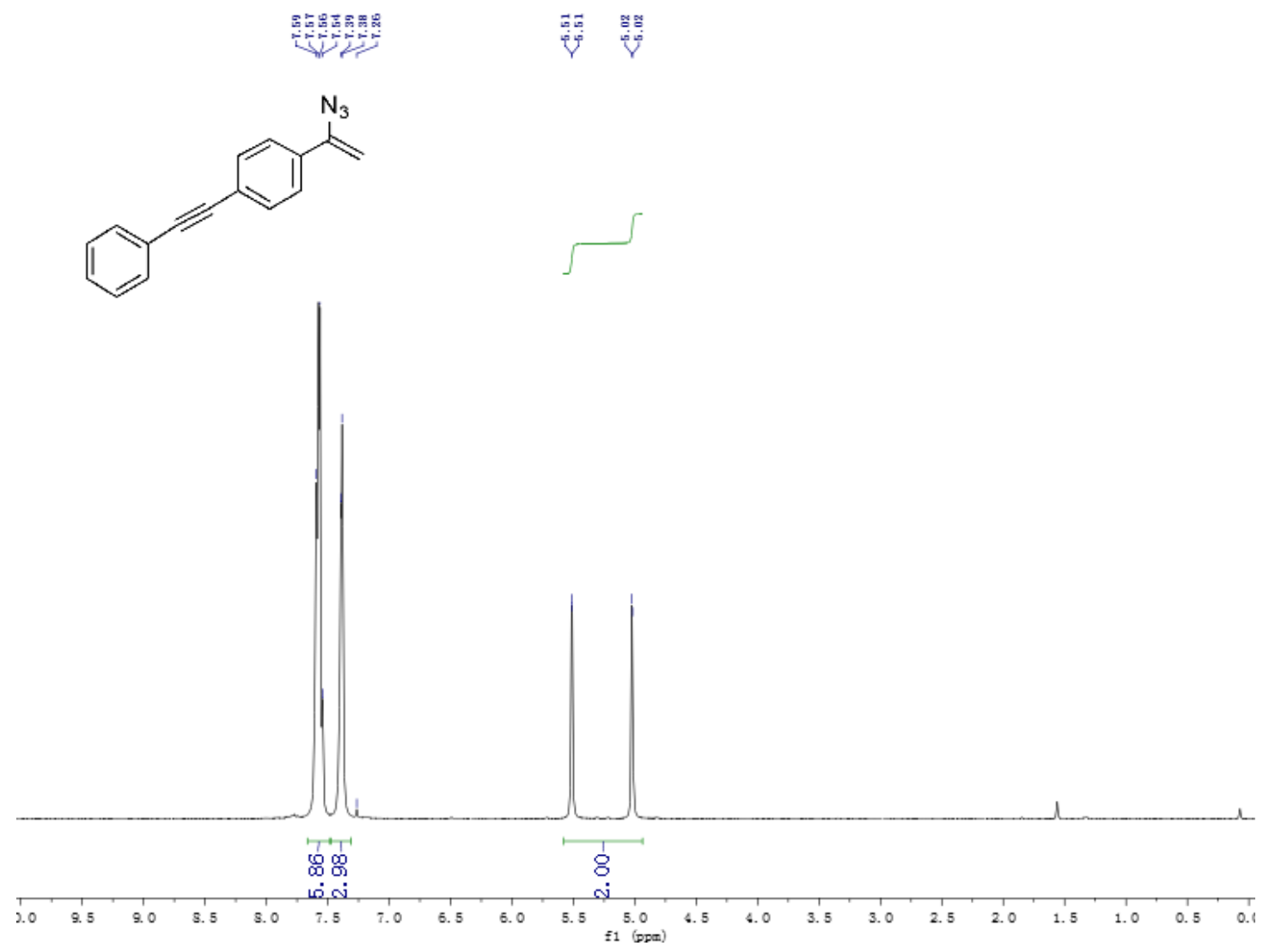

${ }^{13} \mathrm{C}$ NMR of $1 \mathrm{n}$

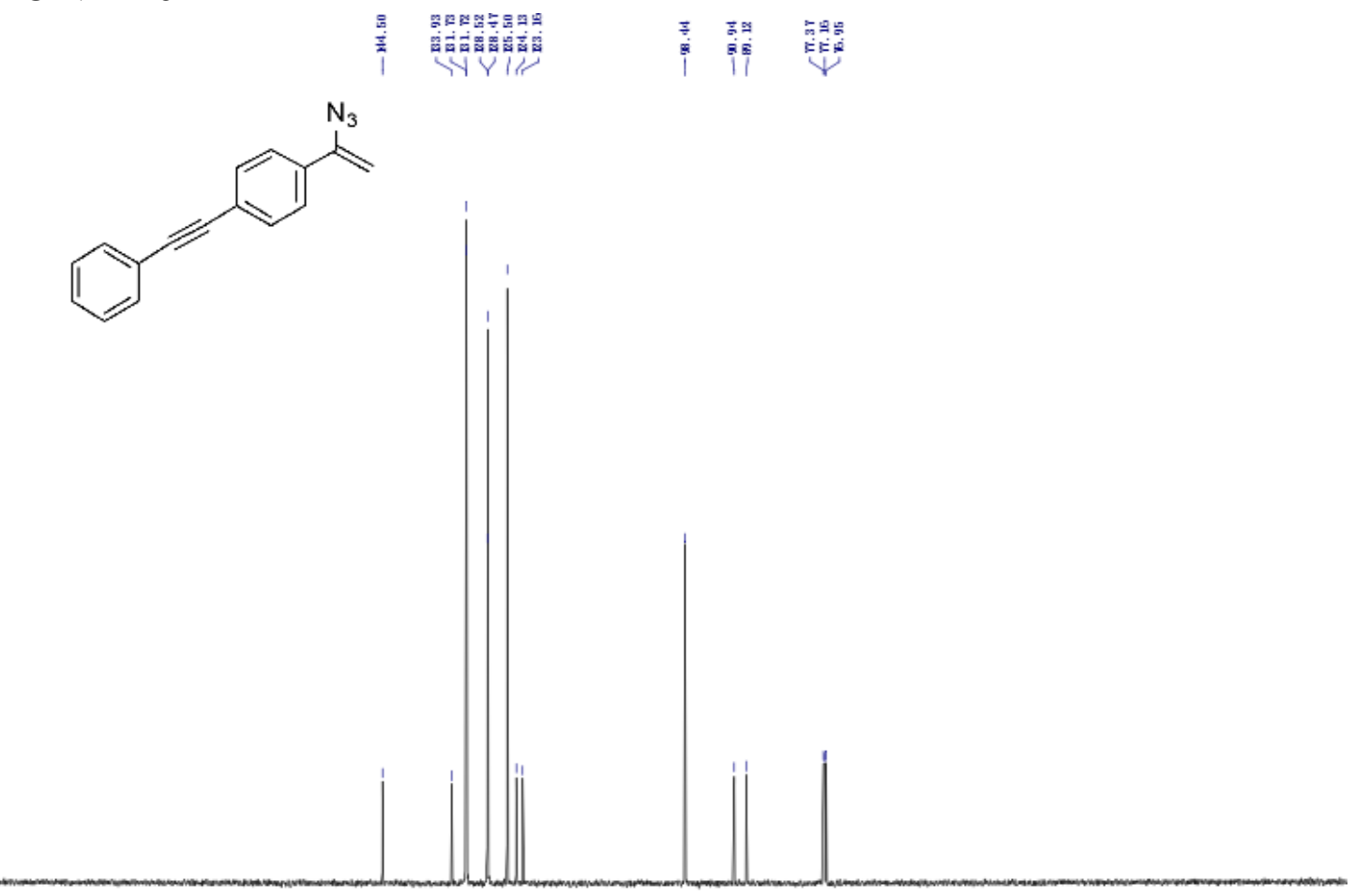

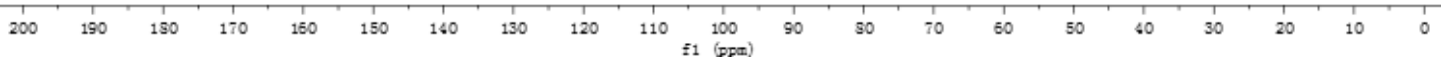


${ }^{1} \mathrm{H}$ NMR of 10

照㽝是

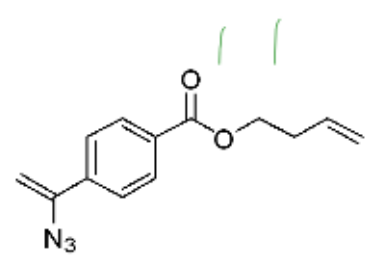

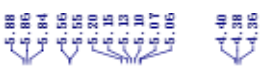

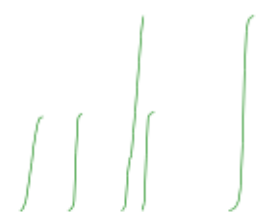

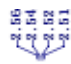

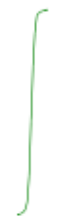

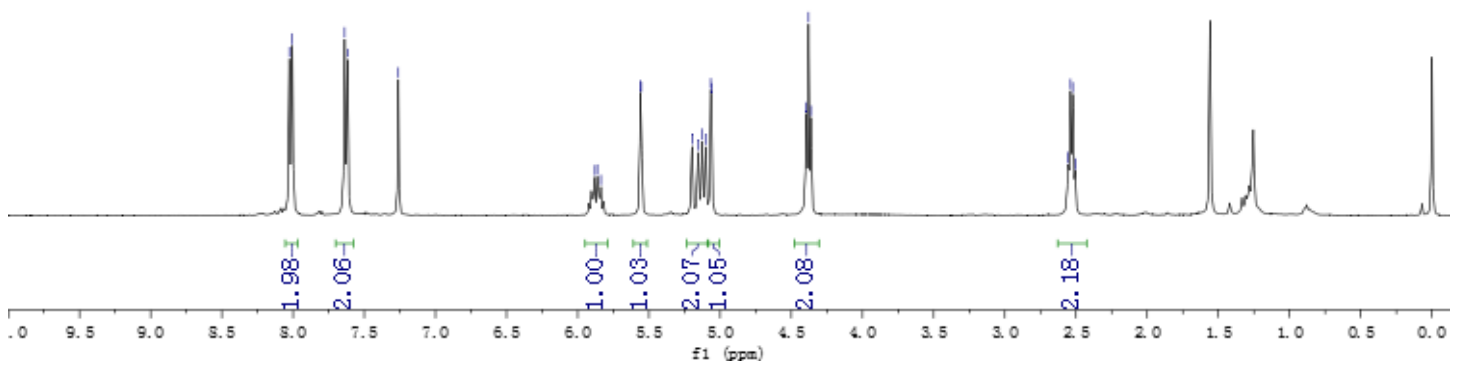

${ }^{13} \mathrm{C}$ NMR of 10
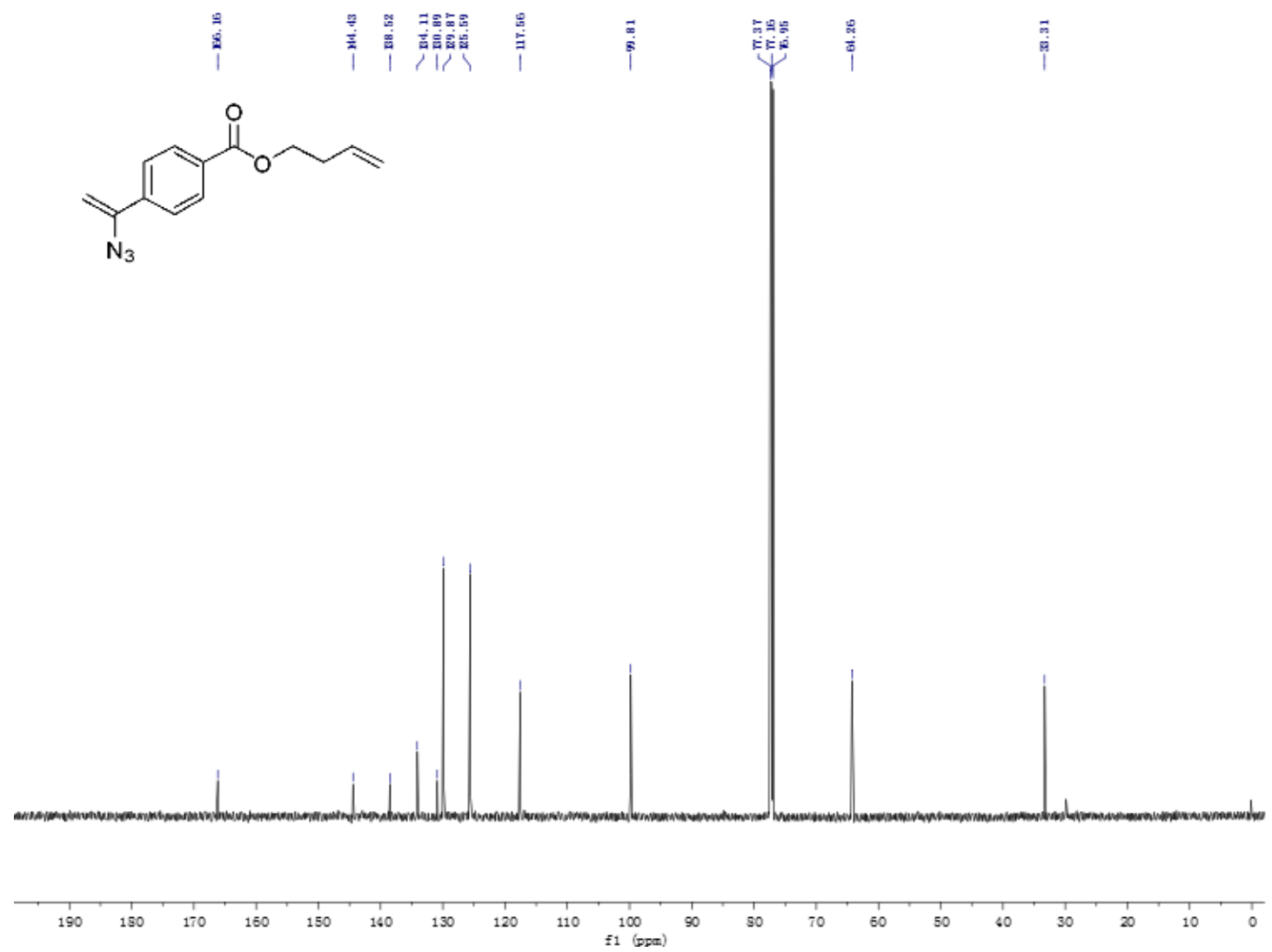

33 
${ }^{1} \mathrm{H}$ NMR of 1p
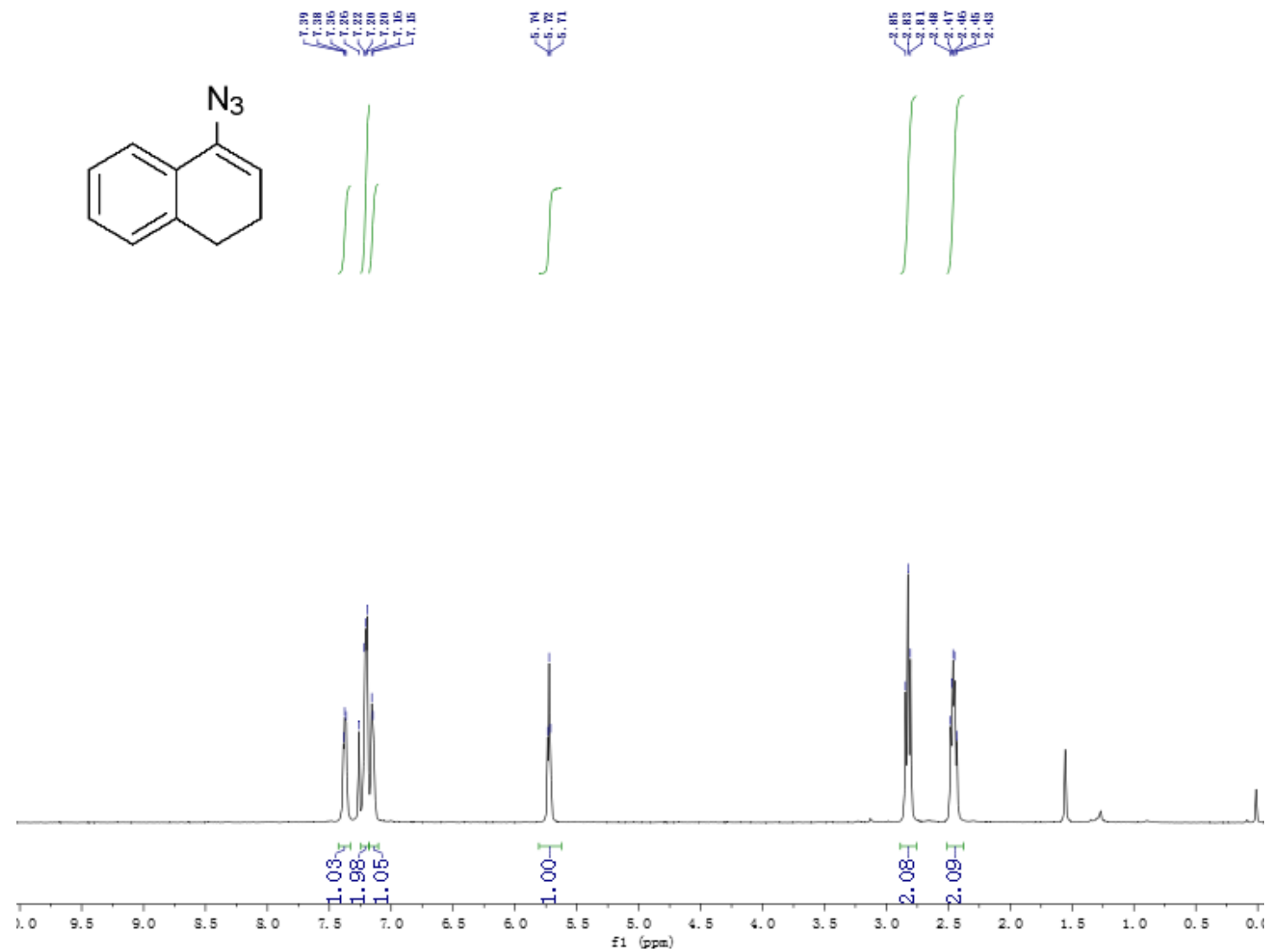

${ }^{13} \mathrm{C}$ NMR of $\mathbf{1 p}$

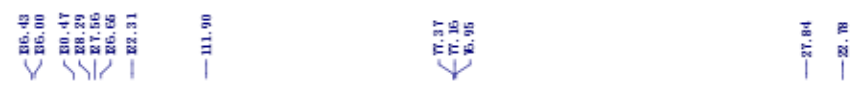<smiles>NC1=CCCc2ccccc21</smiles>

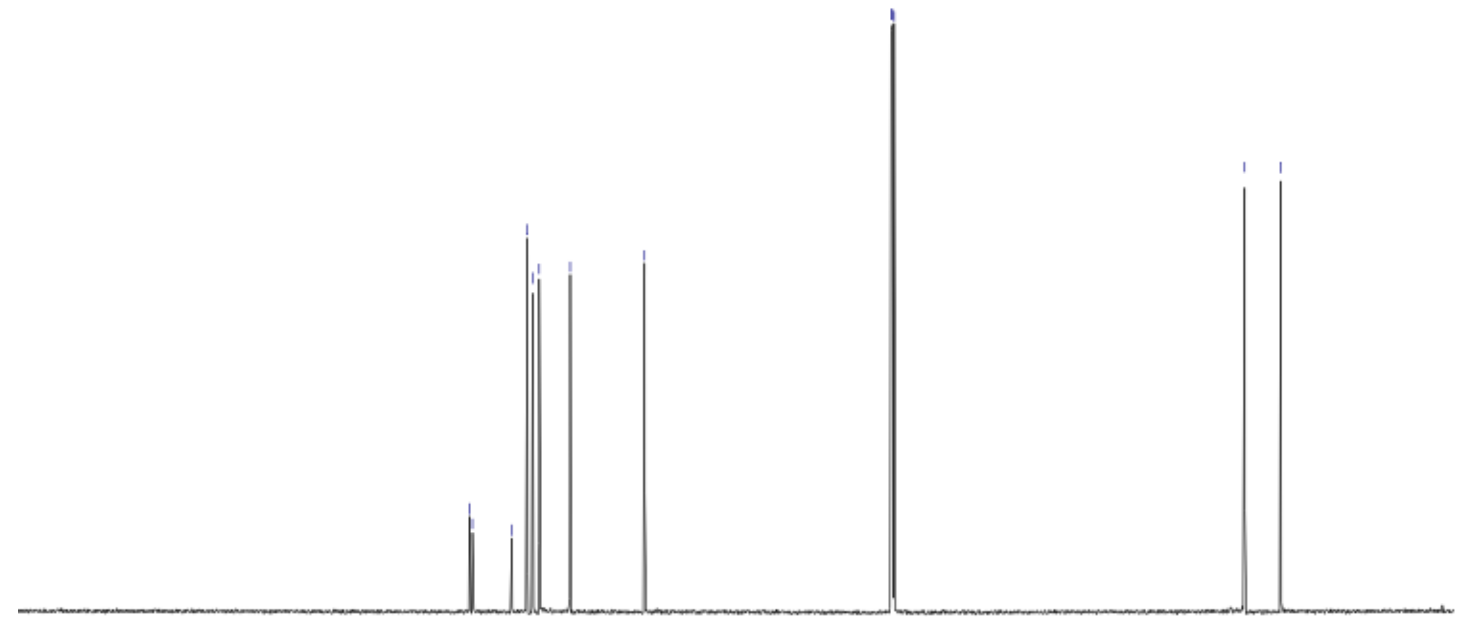

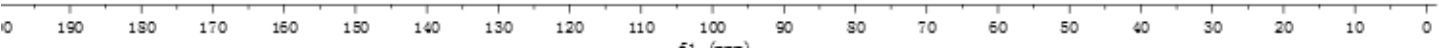


${ }^{1} \mathrm{H}$ NMR of $\mathbf{1 q}$

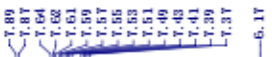<smiles>N/C(=C\c1ccccc1)c1ccccc1</smiles>

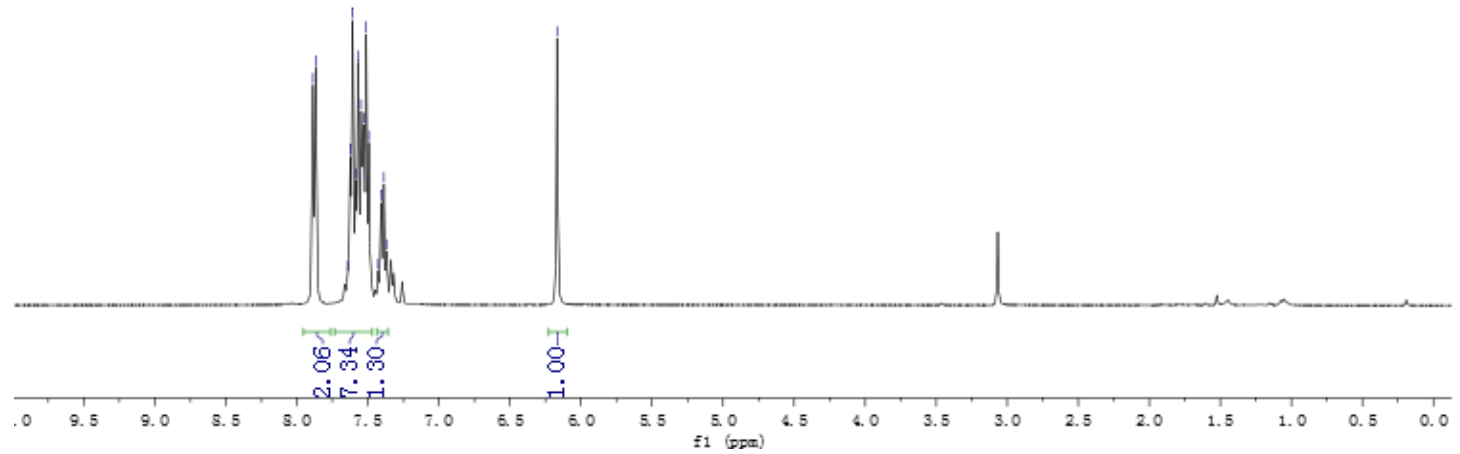

${ }^{13} \mathrm{C}$ NMR of 1q

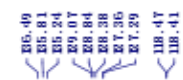

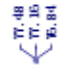
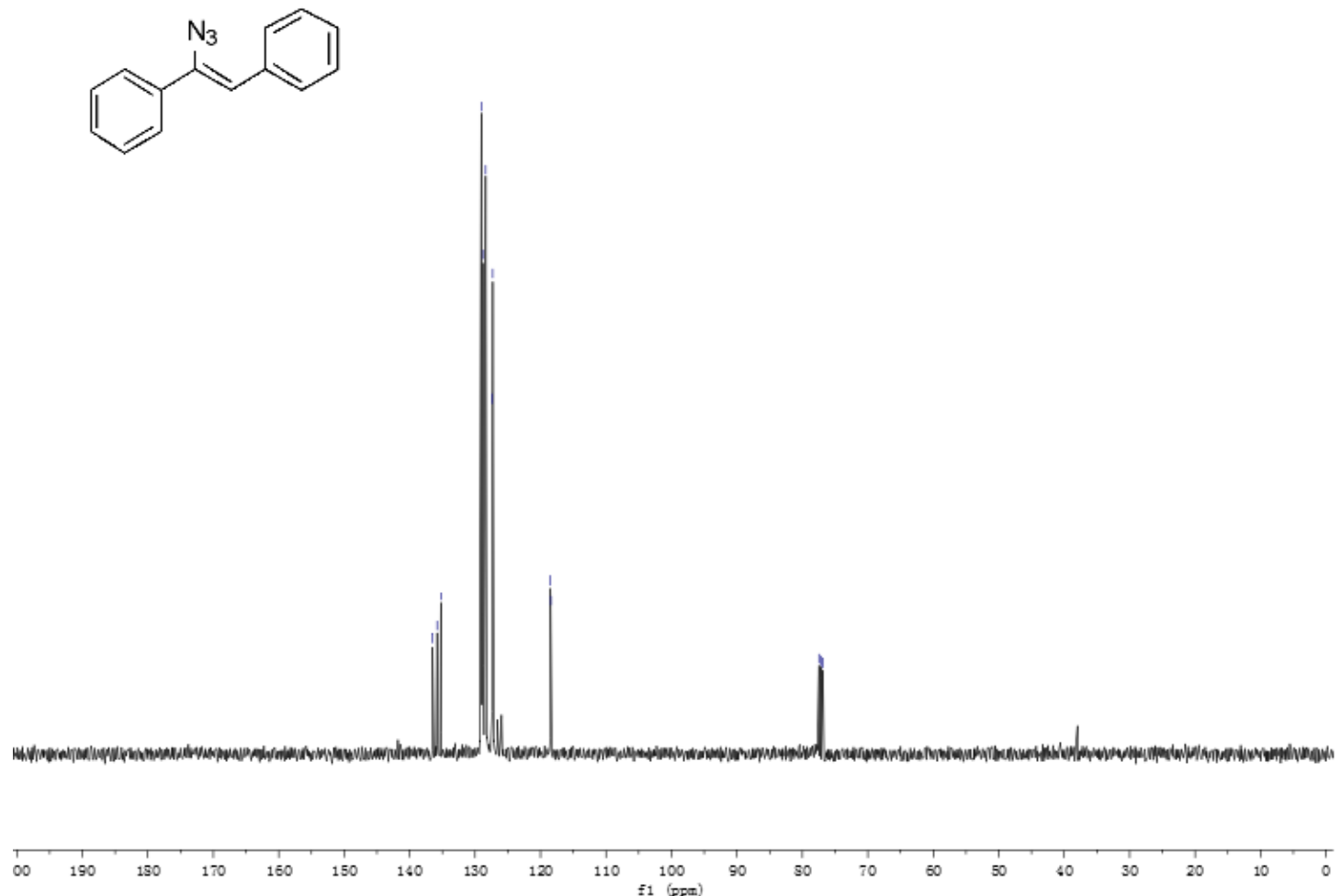
${ }^{1} \mathrm{H}$ NMR of $\mathbf{1 r}$

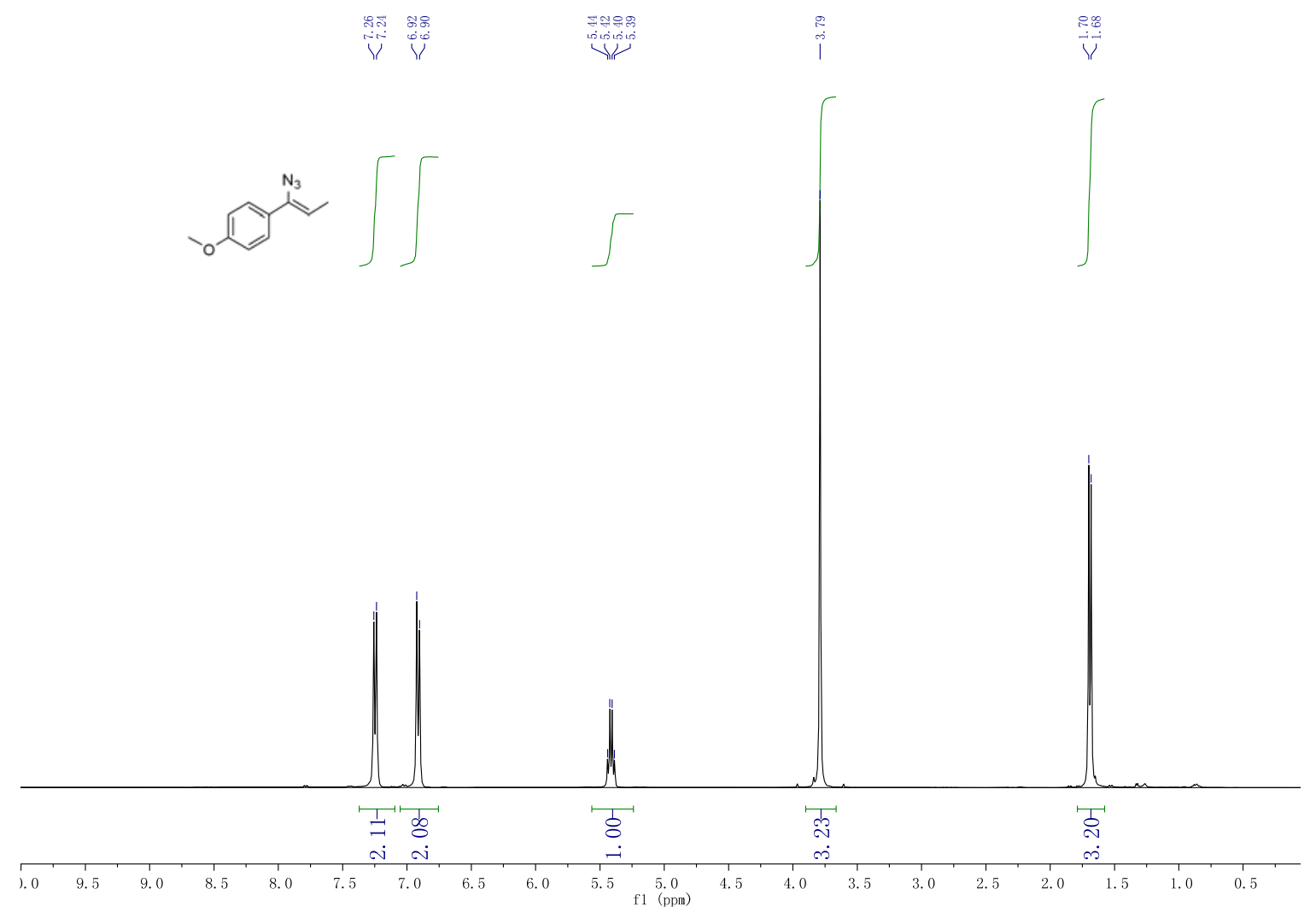

${ }^{13} \mathrm{C}$ NMR of $1 r$

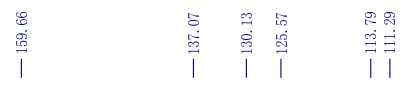

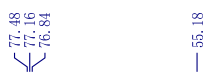<smiles>C/C=C(\N)c1ccc(OC)cc1</smiles>

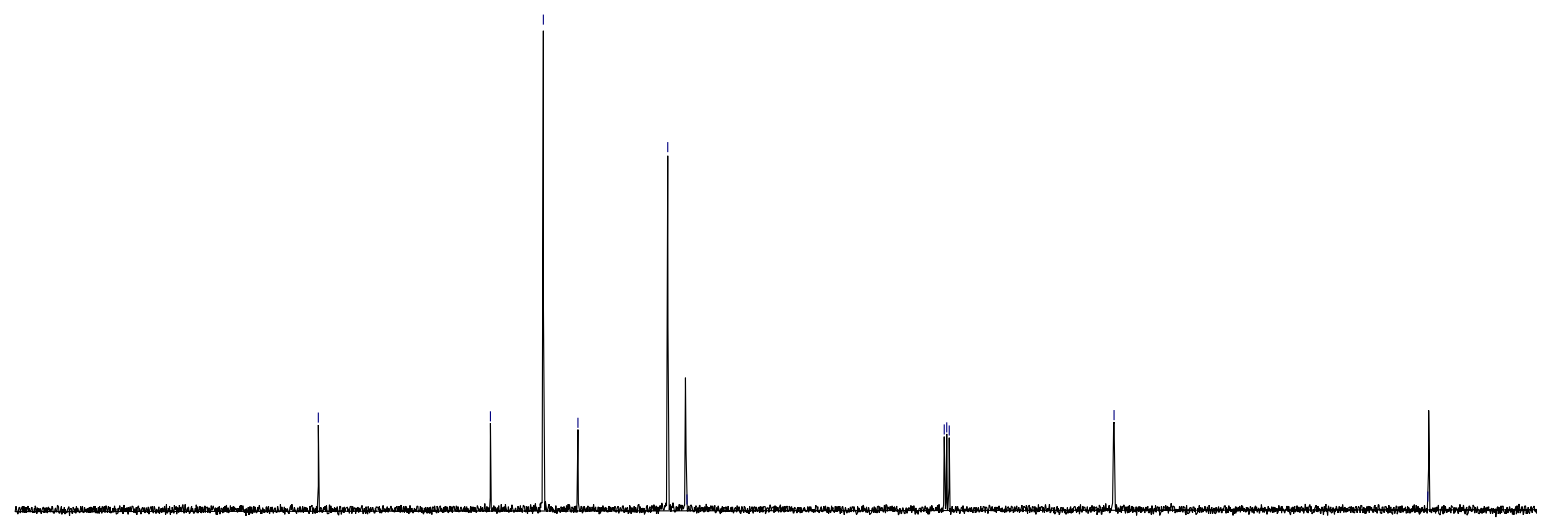

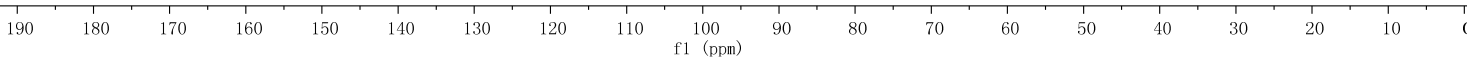


${ }^{1} \mathrm{H}$ NMR of 1 s

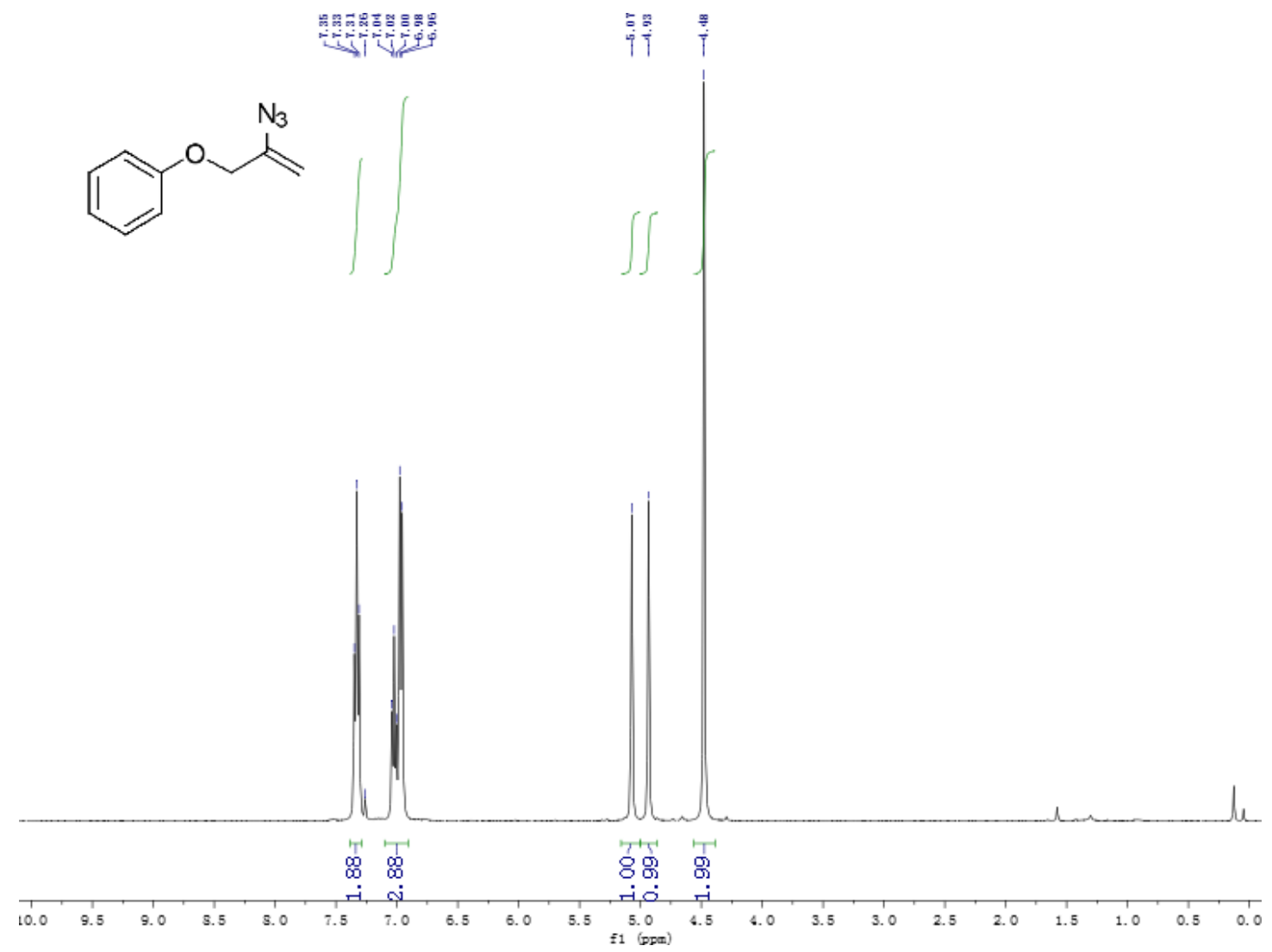

${ }^{13} \mathrm{C}$ NMR of $1 \mathrm{~s}$

采

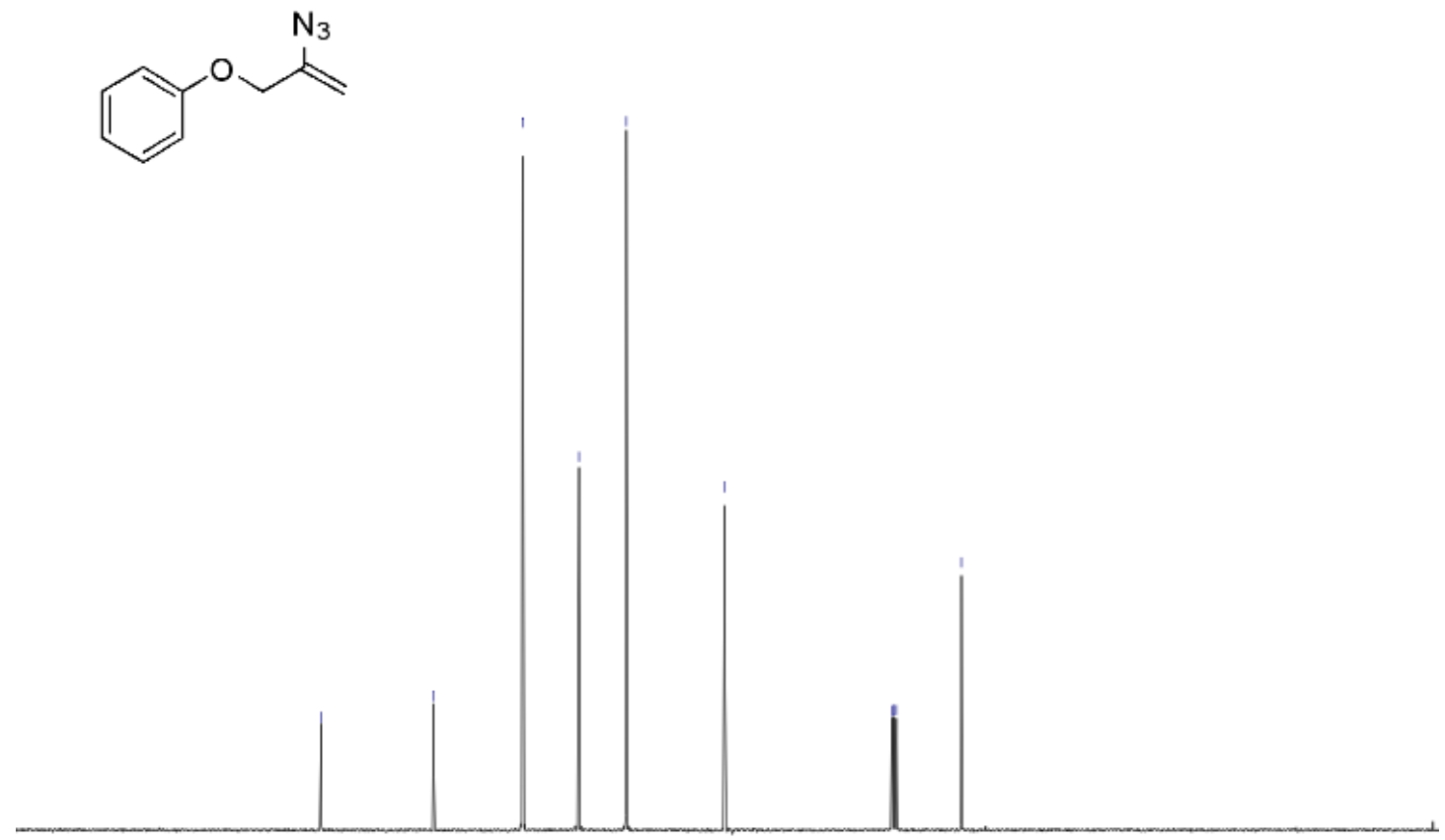

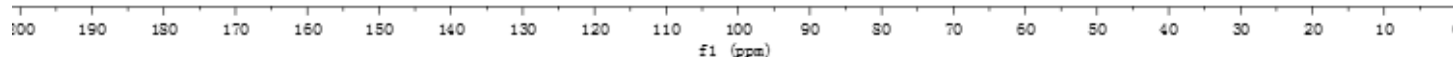


${ }^{1} \mathrm{H}$ NMR of $\mathbf{1 t}$
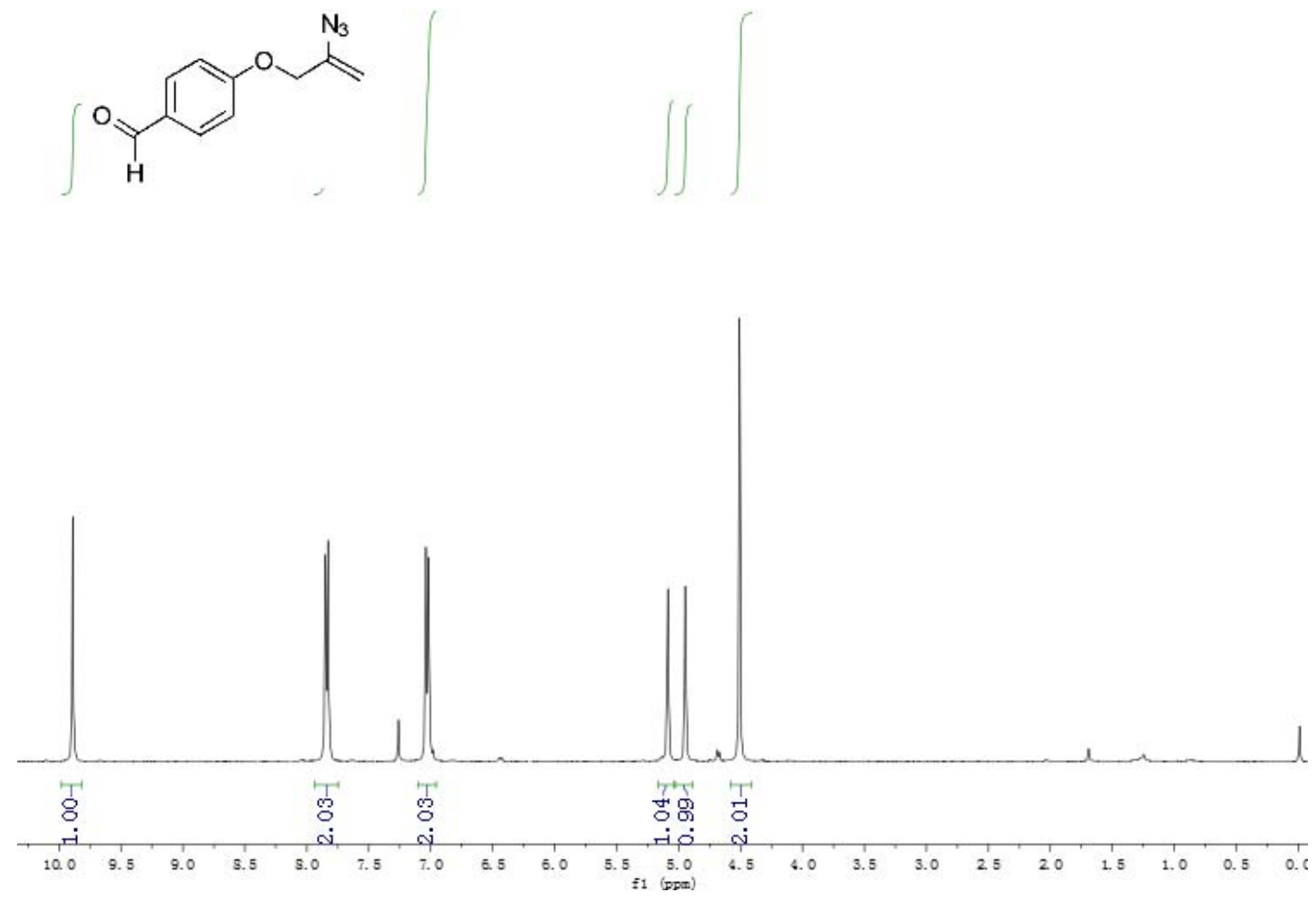

${ }^{13} \mathrm{C}$ NMR of $\mathbf{1 t}$

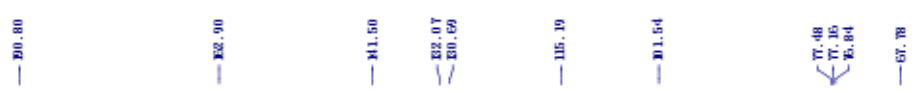

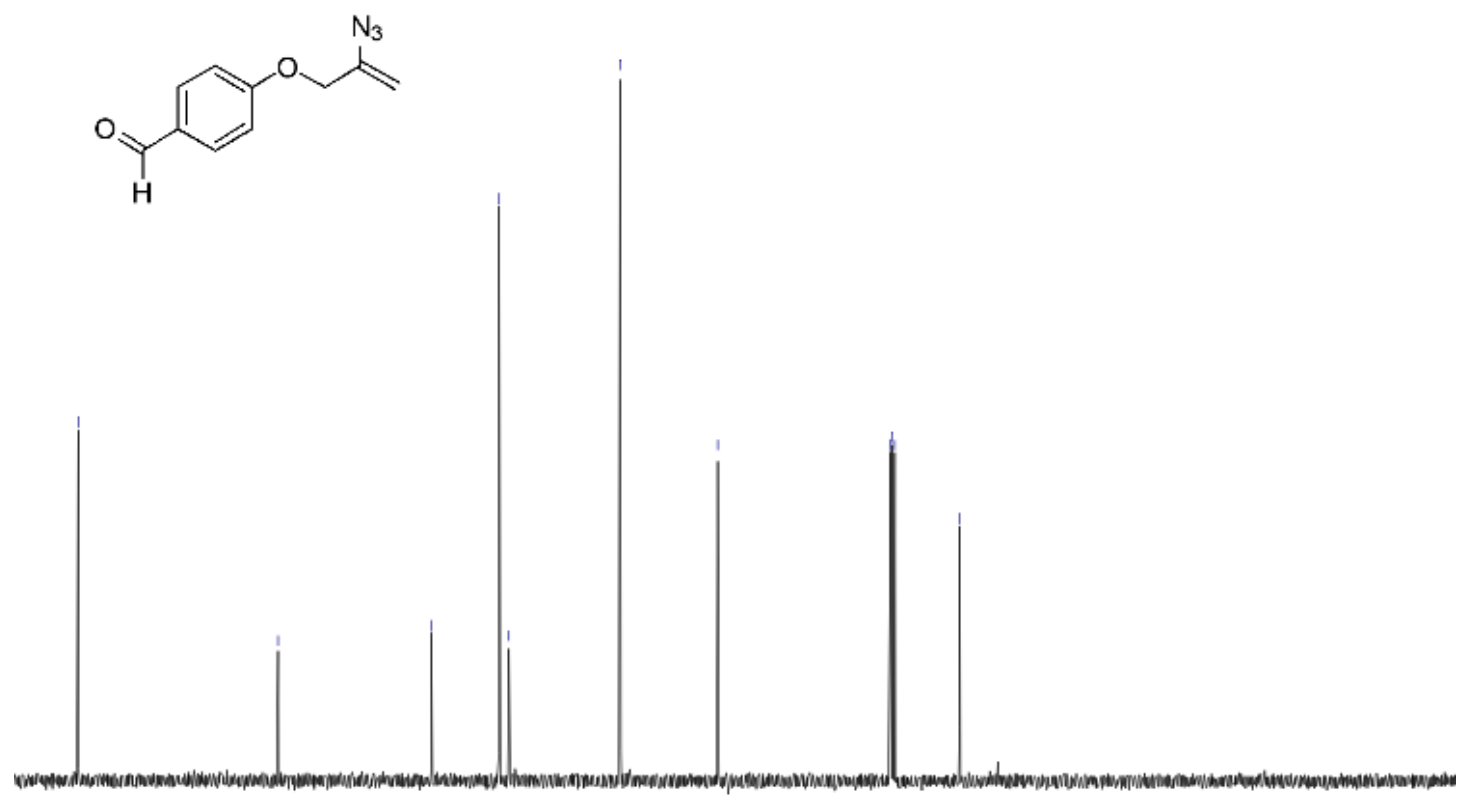

$\begin{array}{lllllllllllllllllllll}1 & 190 & 180 & 170 & 160 & 150 & 140 & 130 & 120 & 110 & 100 & 90 & 50 & 70 & 60 & 50 & 40 & 30 & 20 & 10 & 0\end{array}$ 
${ }^{1} \mathrm{H}$ NMR of $\mathbf{1 u}$

骨臹

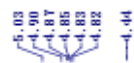

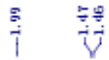<smiles>C=C(N)COc1ccc(C(C)O)cc1</smiles>
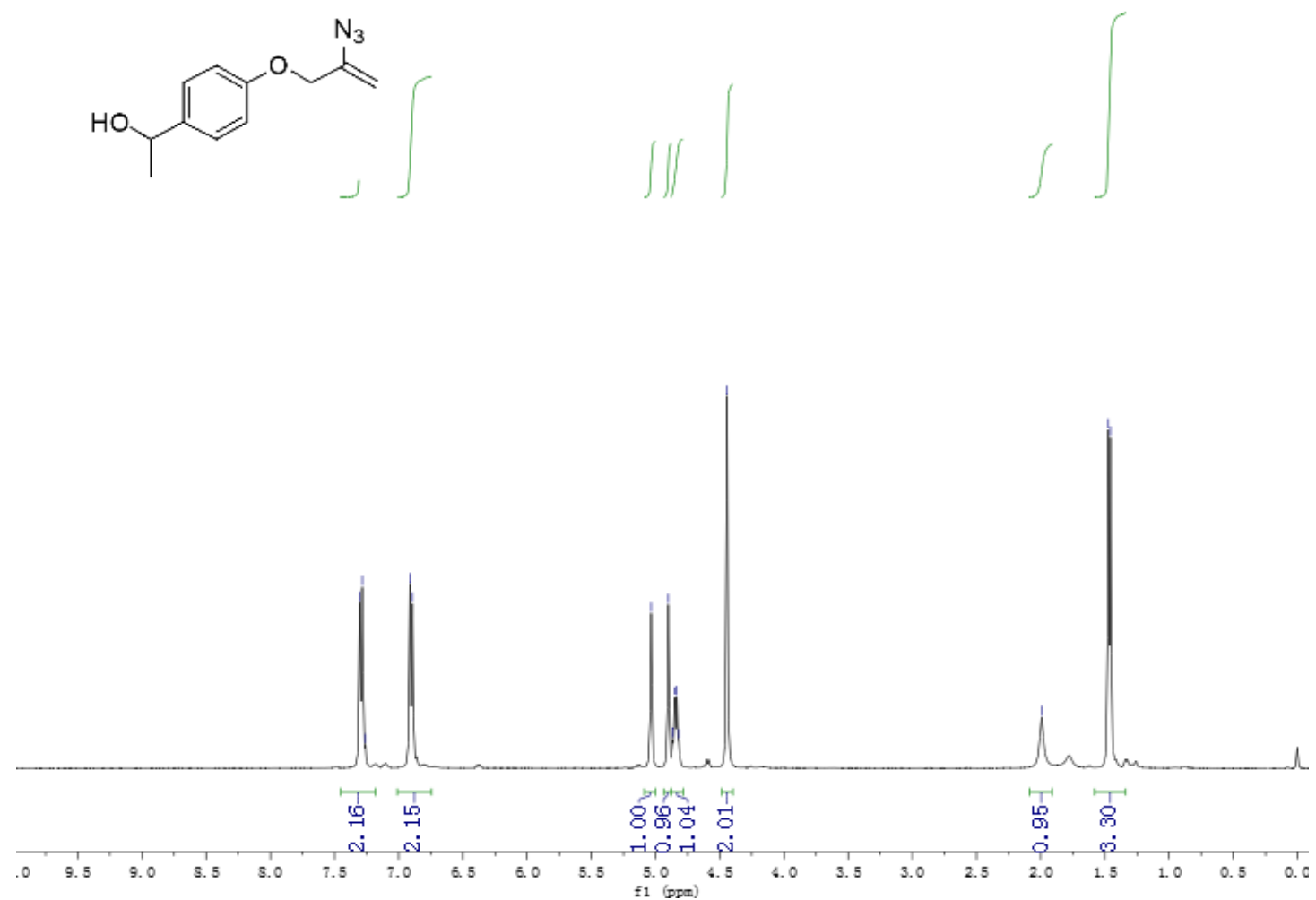

${ }^{13} \mathrm{C}$ NMR of $\mathbf{1 u}$

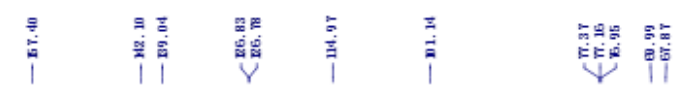

$\stackrel{\bar{u}}{i}$<smiles>C=C(N)COc1ccc(C(C)O)cc1</smiles>

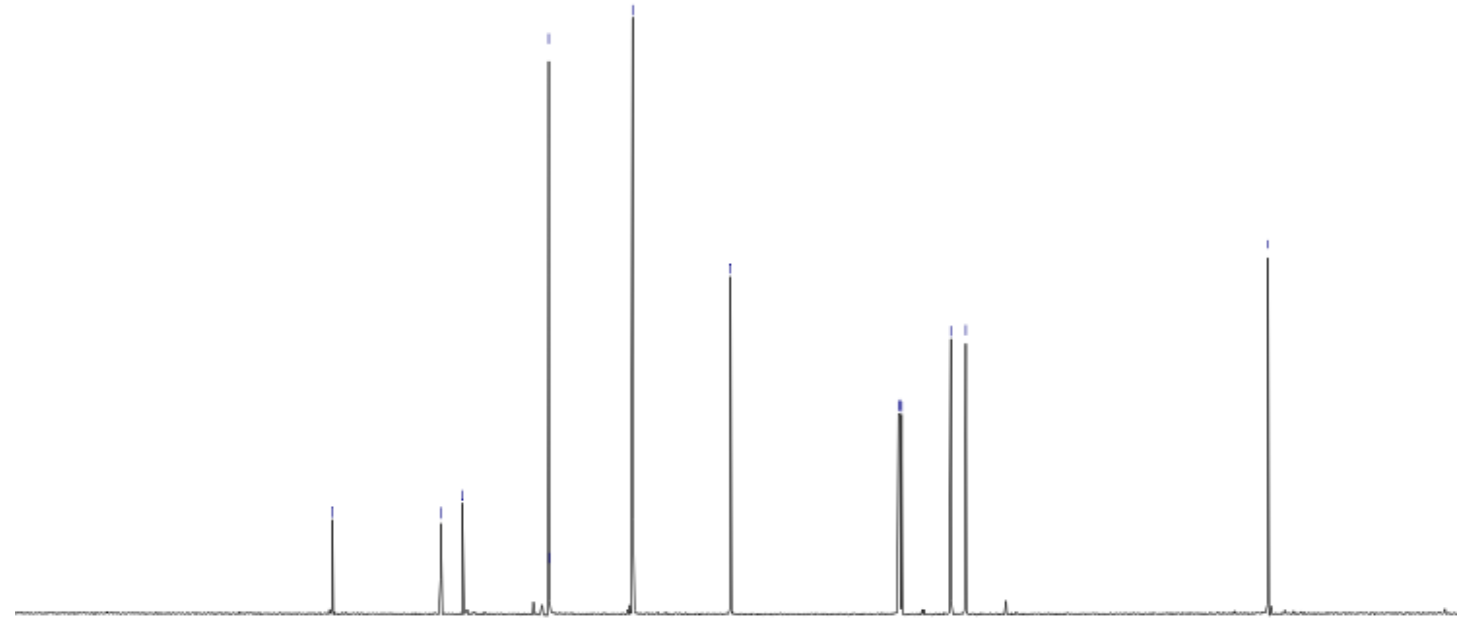

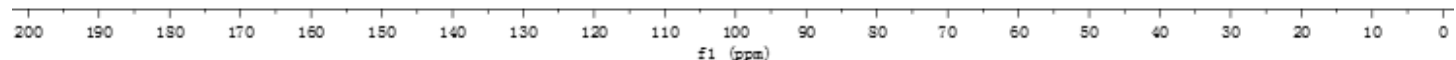


${ }^{1} \mathrm{H}$ NMR of $\mathbf{1 v}$

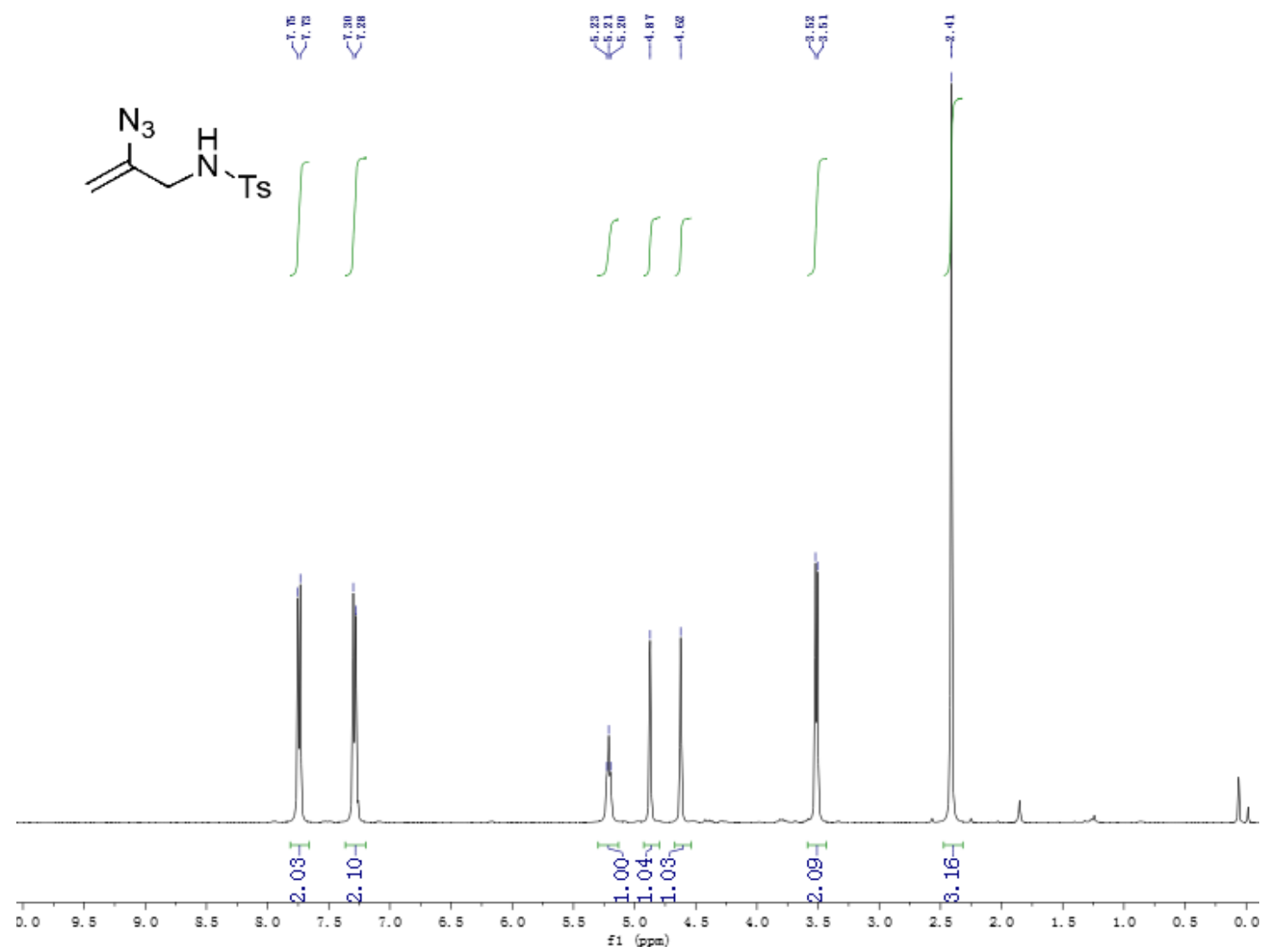

${ }^{13} \mathrm{C}$ NMR of $1 \mathbf{v}$

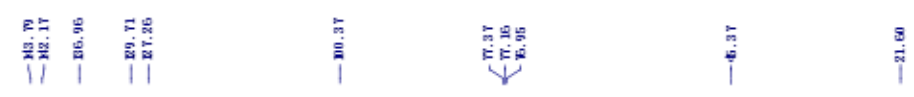

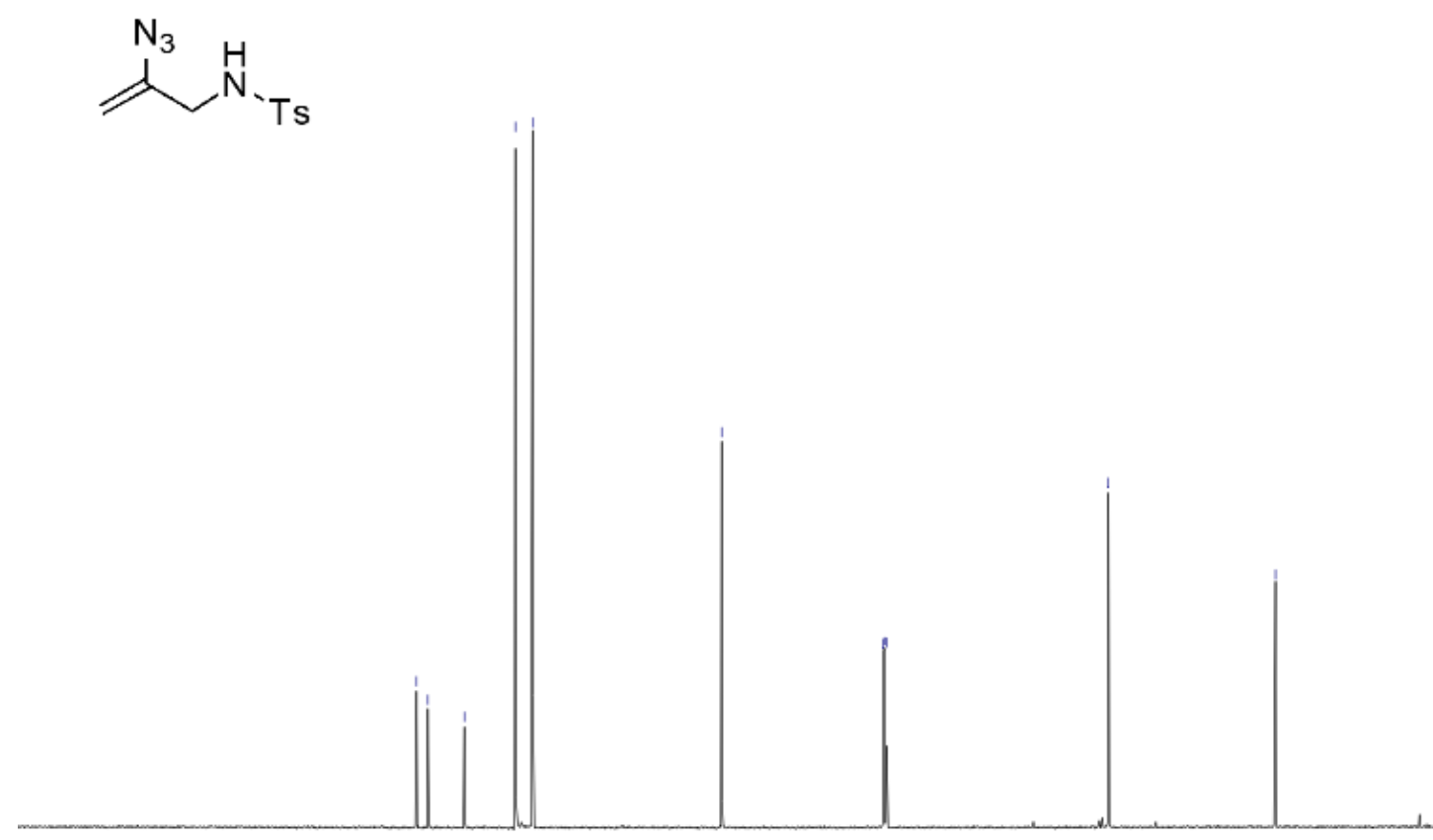

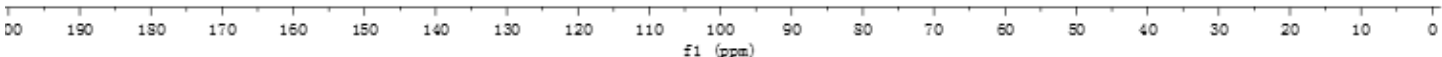


${ }^{1} \mathrm{H}$ NMR of $\mathbf{1 w}$
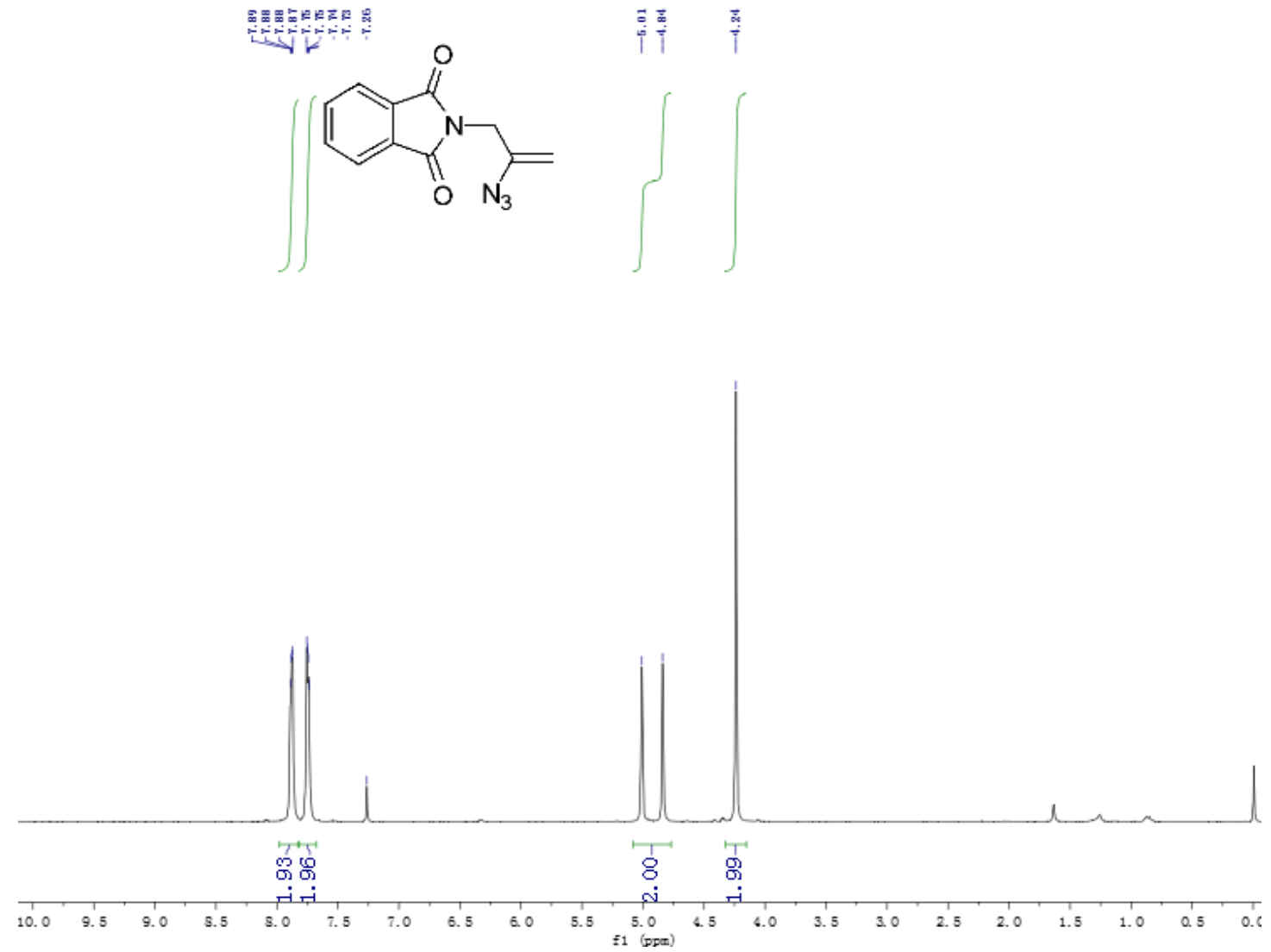

${ }^{13} \mathrm{C}$ NMR of $\mathbf{1 w}$

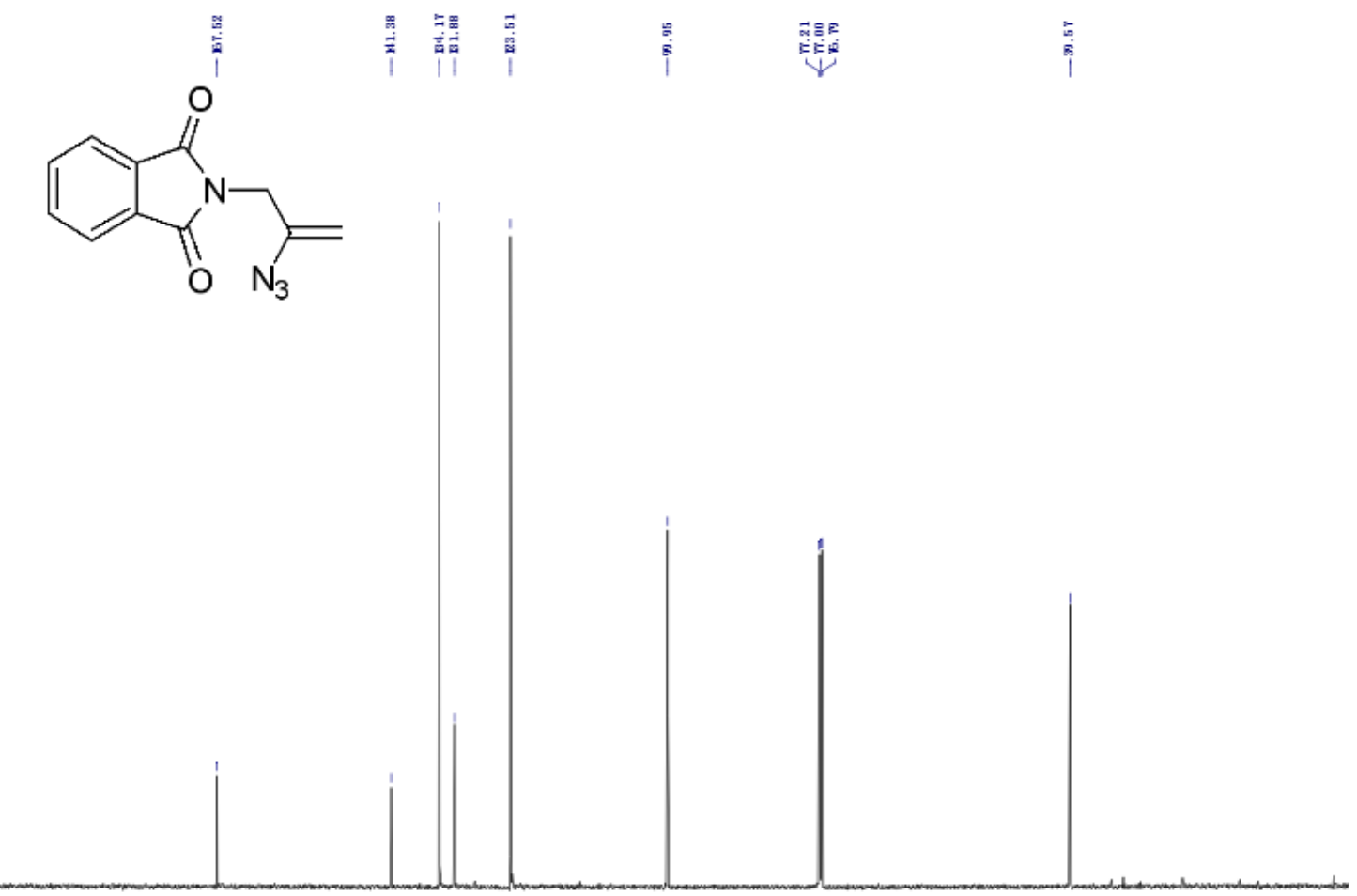

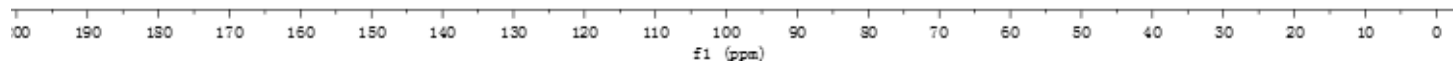


${ }^{1} \mathrm{H}$ NMR of $\mathbf{1 x}$
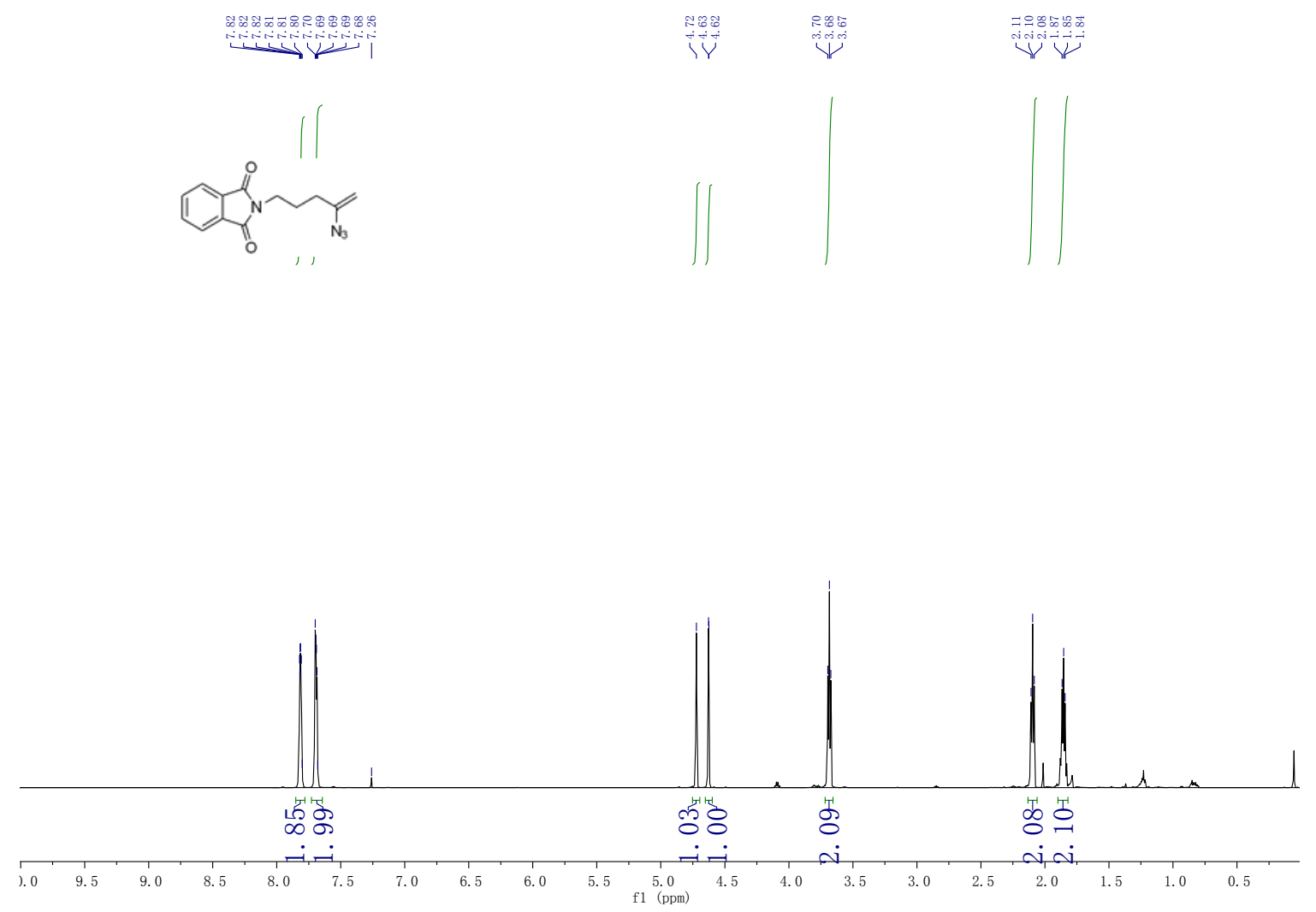

${ }^{13} \mathrm{C}$ NMR of $\mathbf{1 x}$
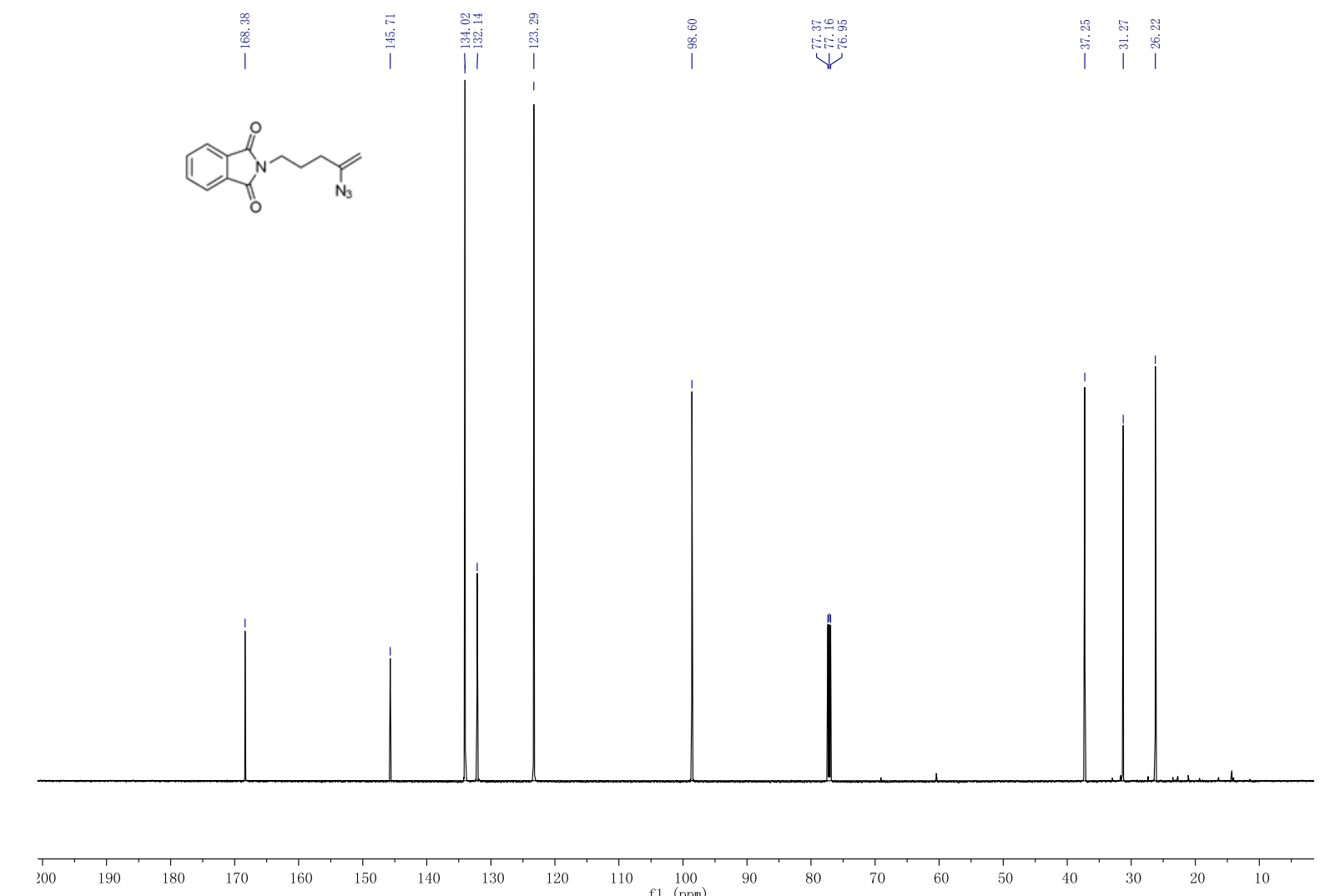
${ }^{1} \mathrm{H}$ NMR of $\mathbf{1 y}$

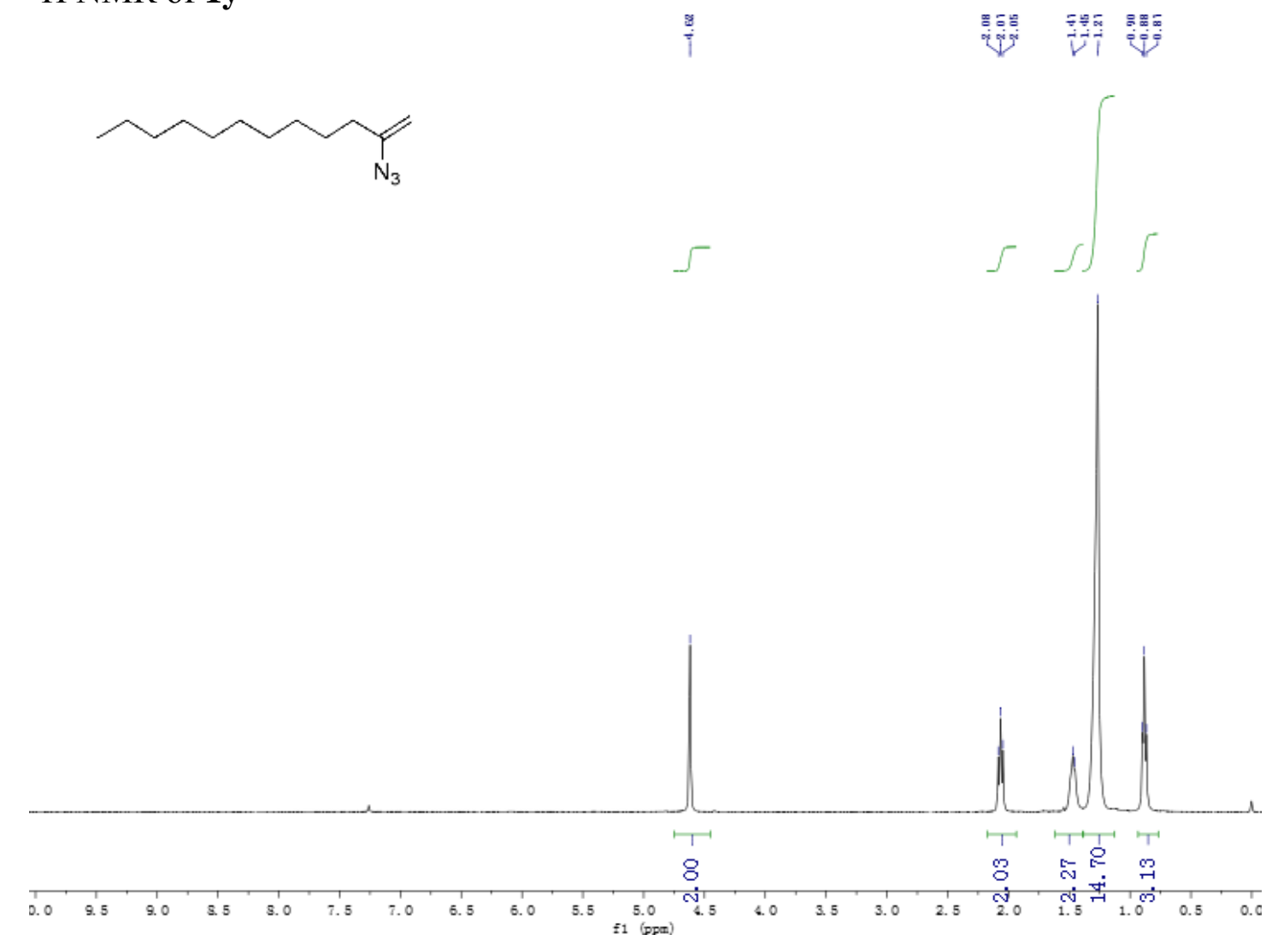

${ }^{13} \mathrm{C}$ NMR of $\mathbf{1 y}$
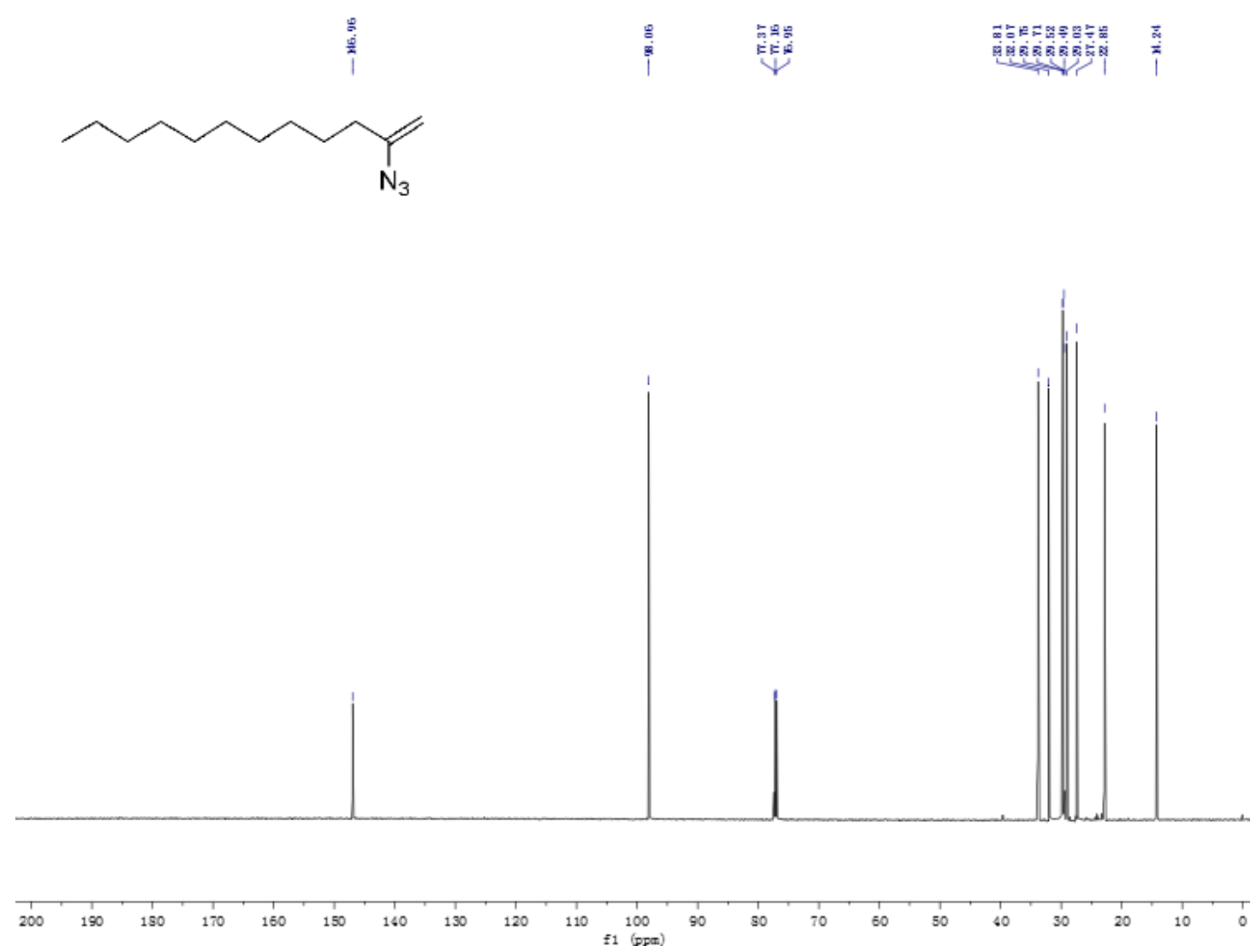
${ }^{1} \mathrm{H}$ NMR of $\mathbf{1 z}$

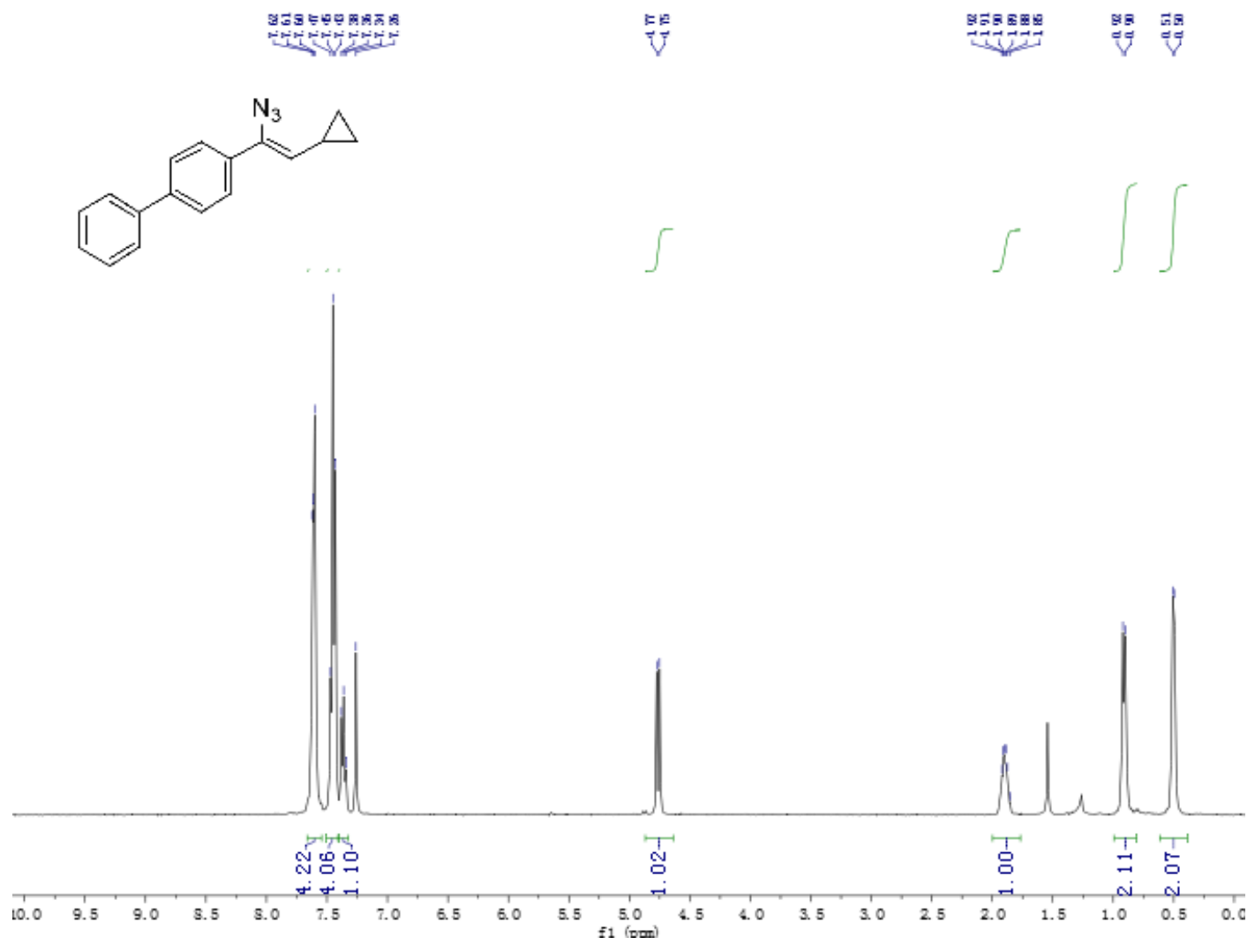

${ }^{13} \mathrm{C}$ NMR of $1 z$

ํㅏㄴ

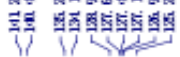

紫里递

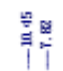<smiles>NC(=CC1CC1)c1ccc(-c2ccccc2)cc1</smiles>

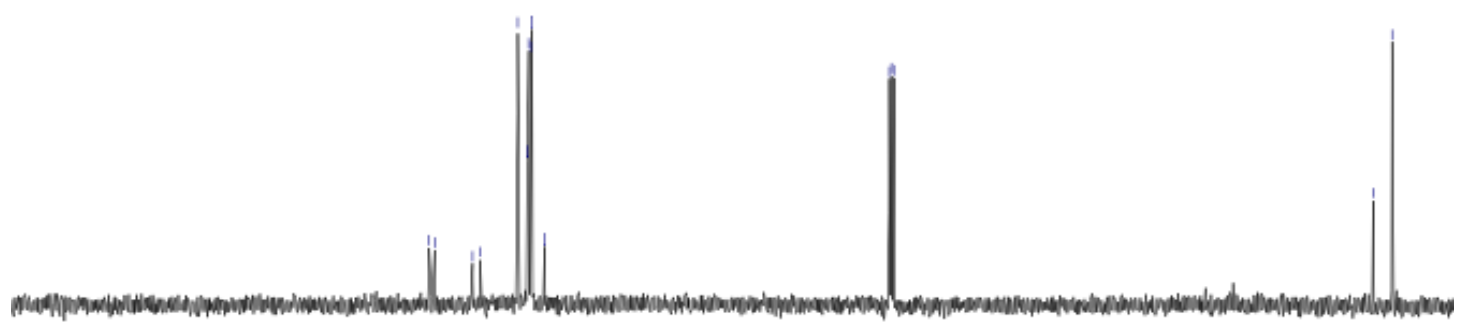

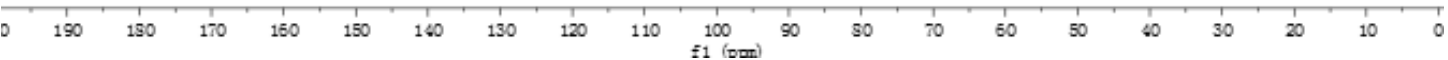


${ }^{1} \mathrm{H}$ NMR of $\mathbf{2 a}$

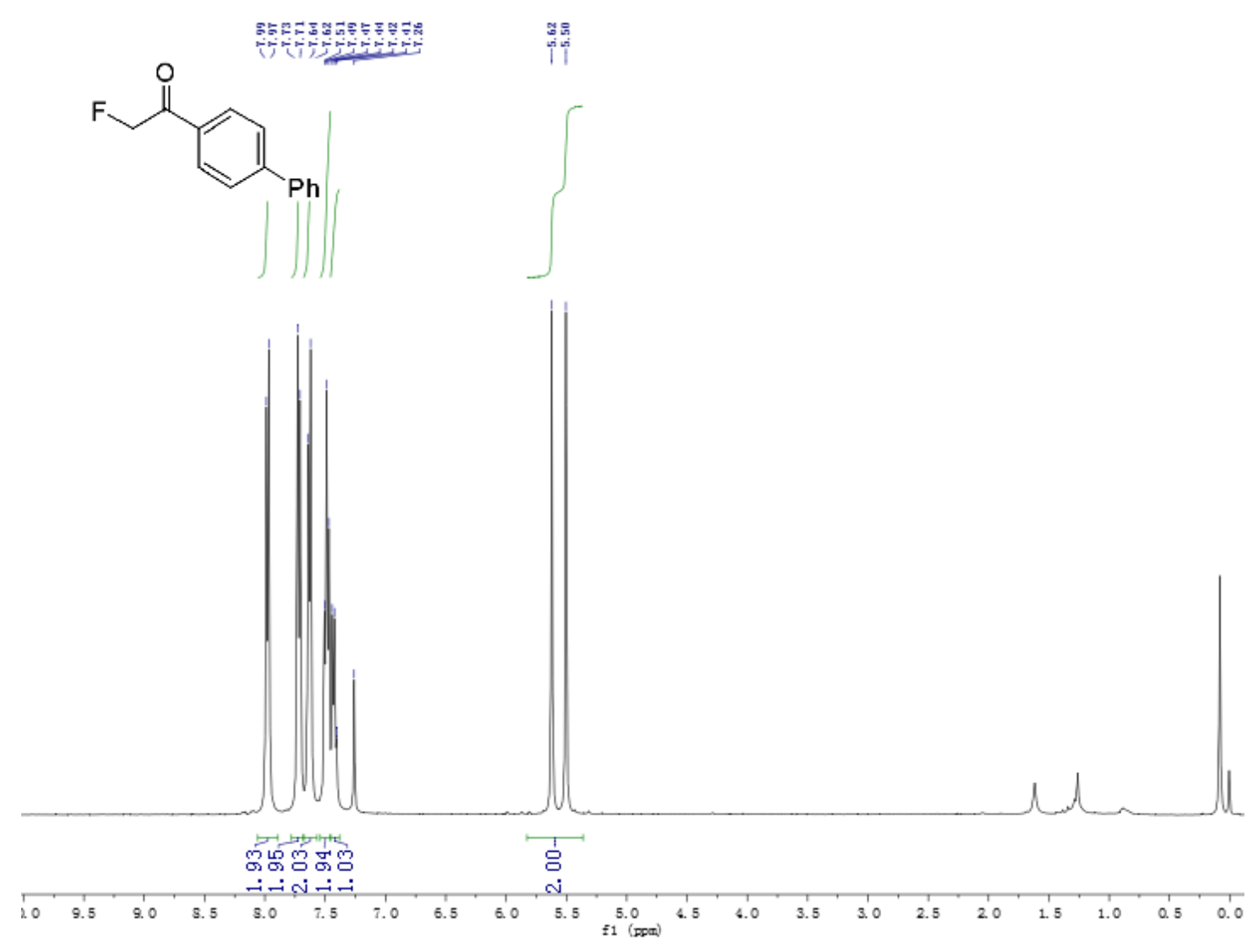

${ }^{13} \mathrm{C}$ NMR of $\mathbf{2 a}$
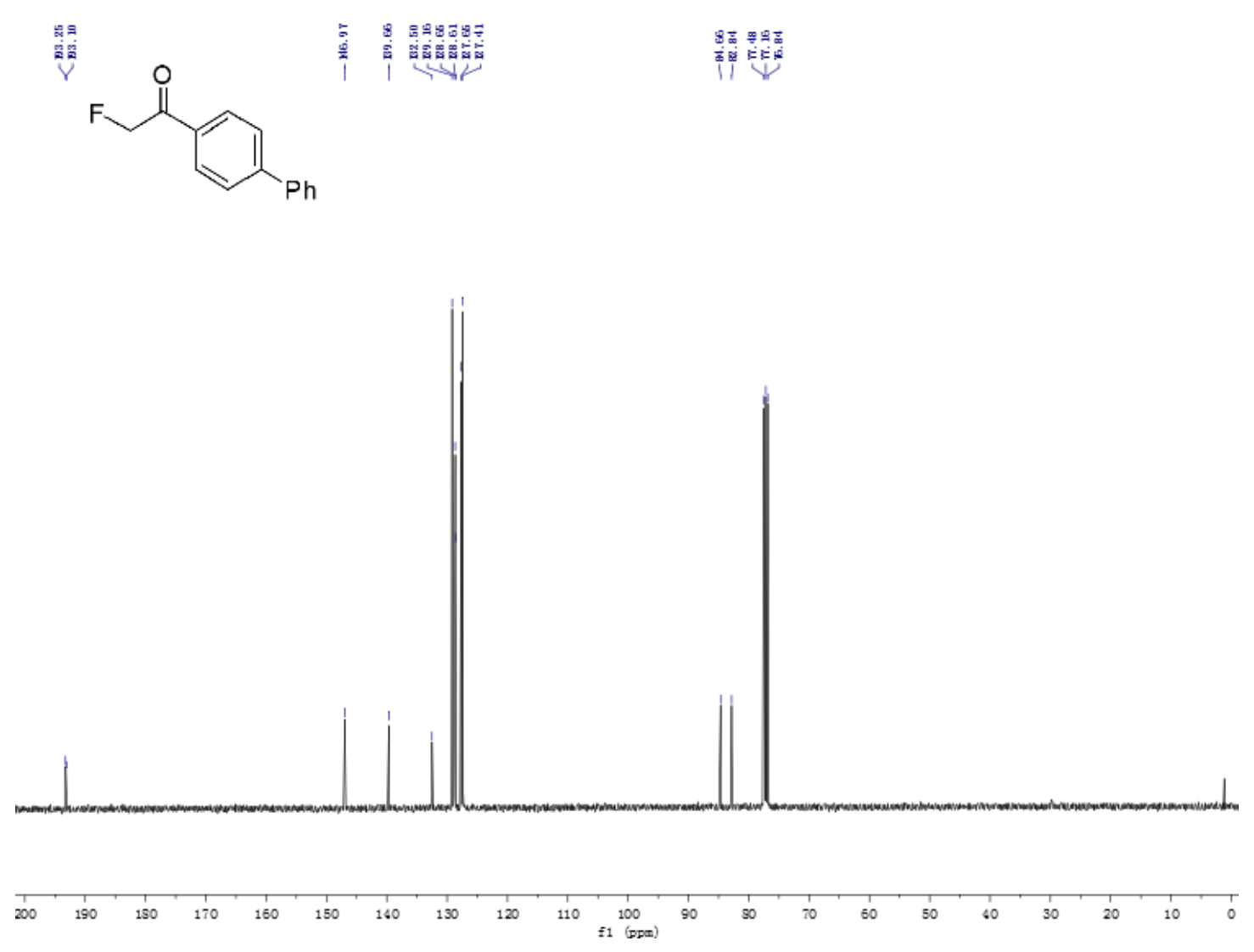

45 
${ }^{19} \mathrm{~F}$ NMR of $\mathbf{2 a}$

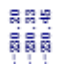
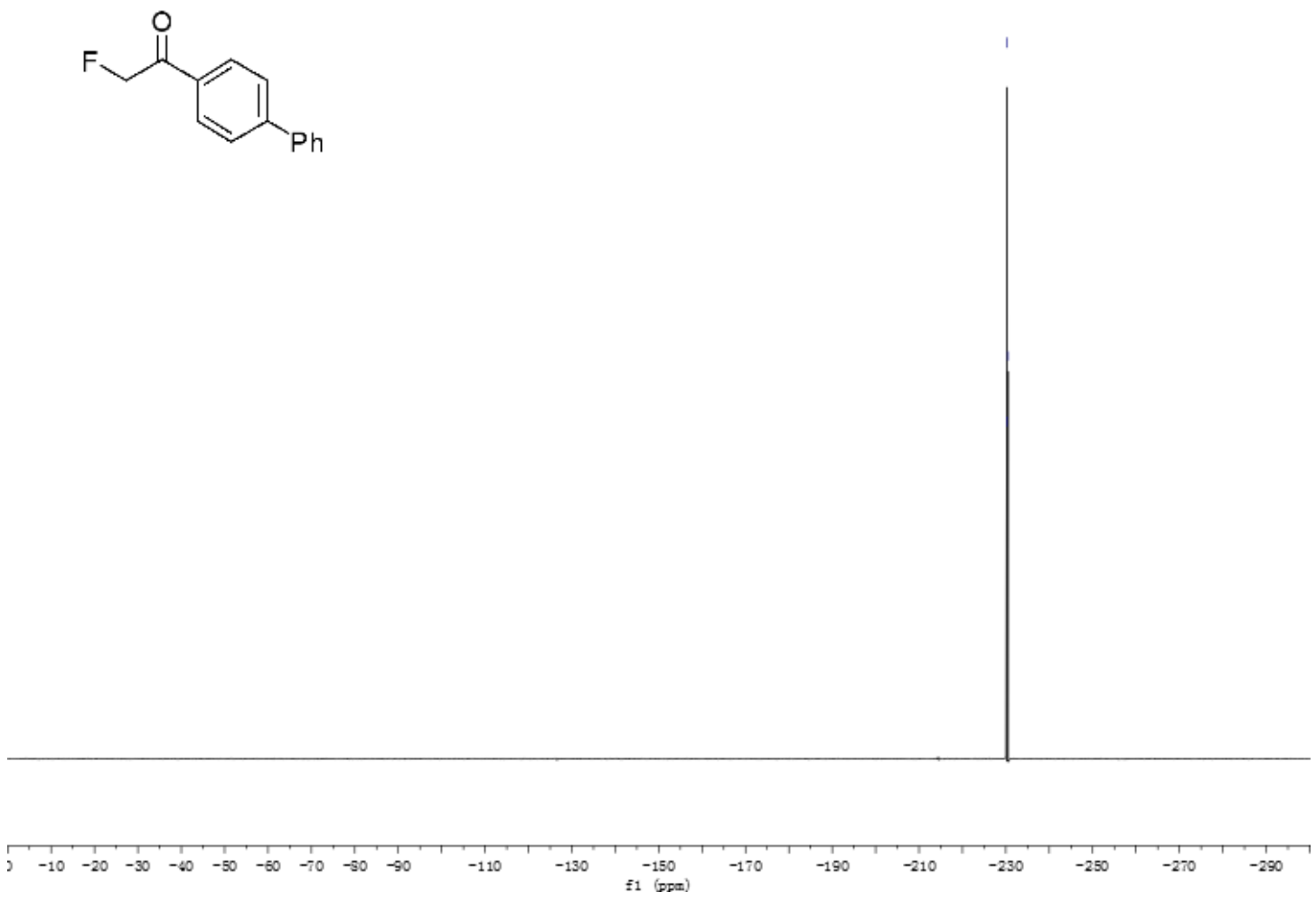

${ }^{1} \mathrm{H}$ NMR of $\mathbf{2 b}$
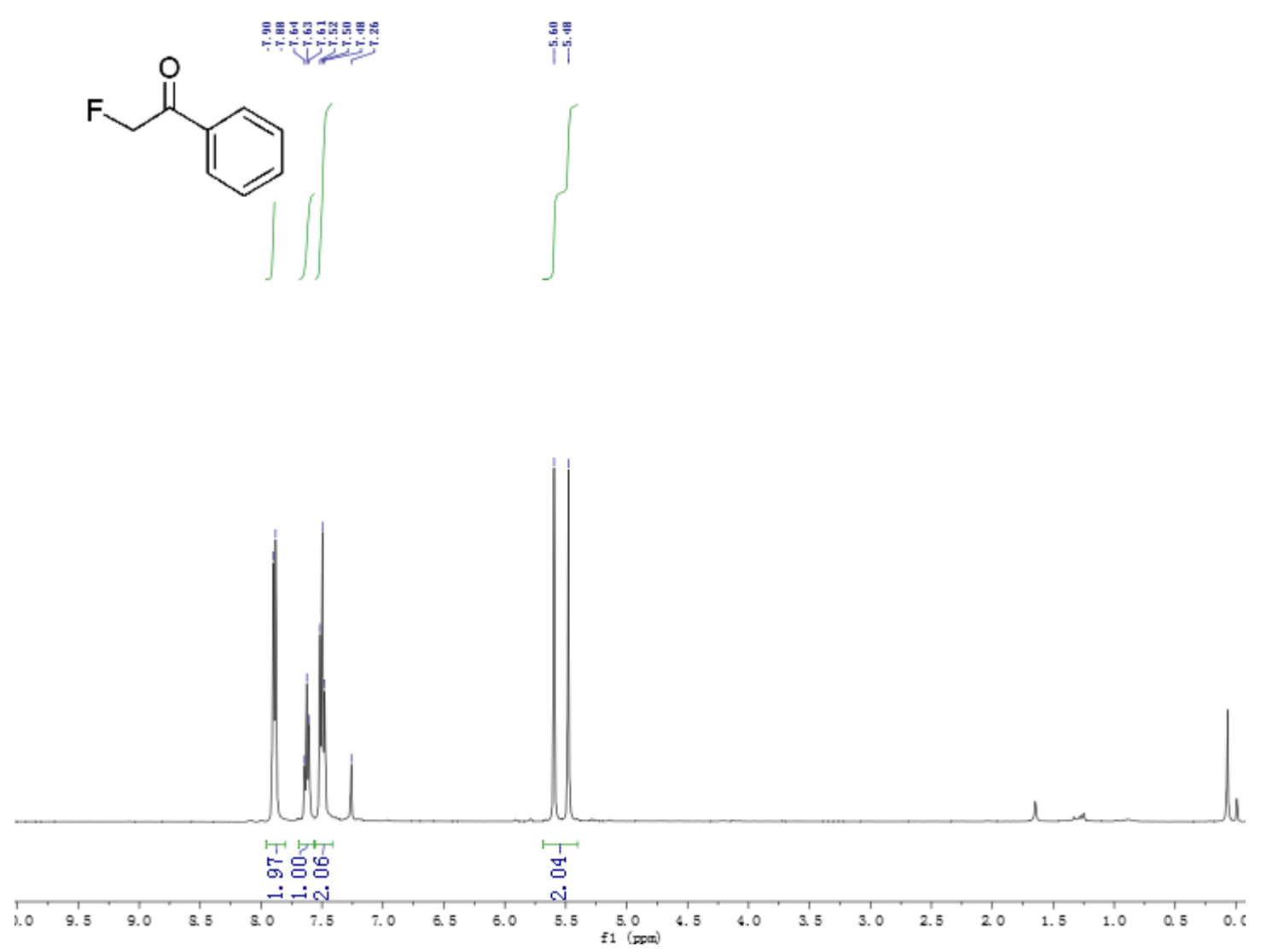

46 
${ }^{13} \mathrm{C}$ NMR of $\mathbf{2 b}$

步埇

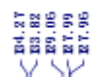

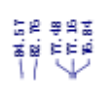
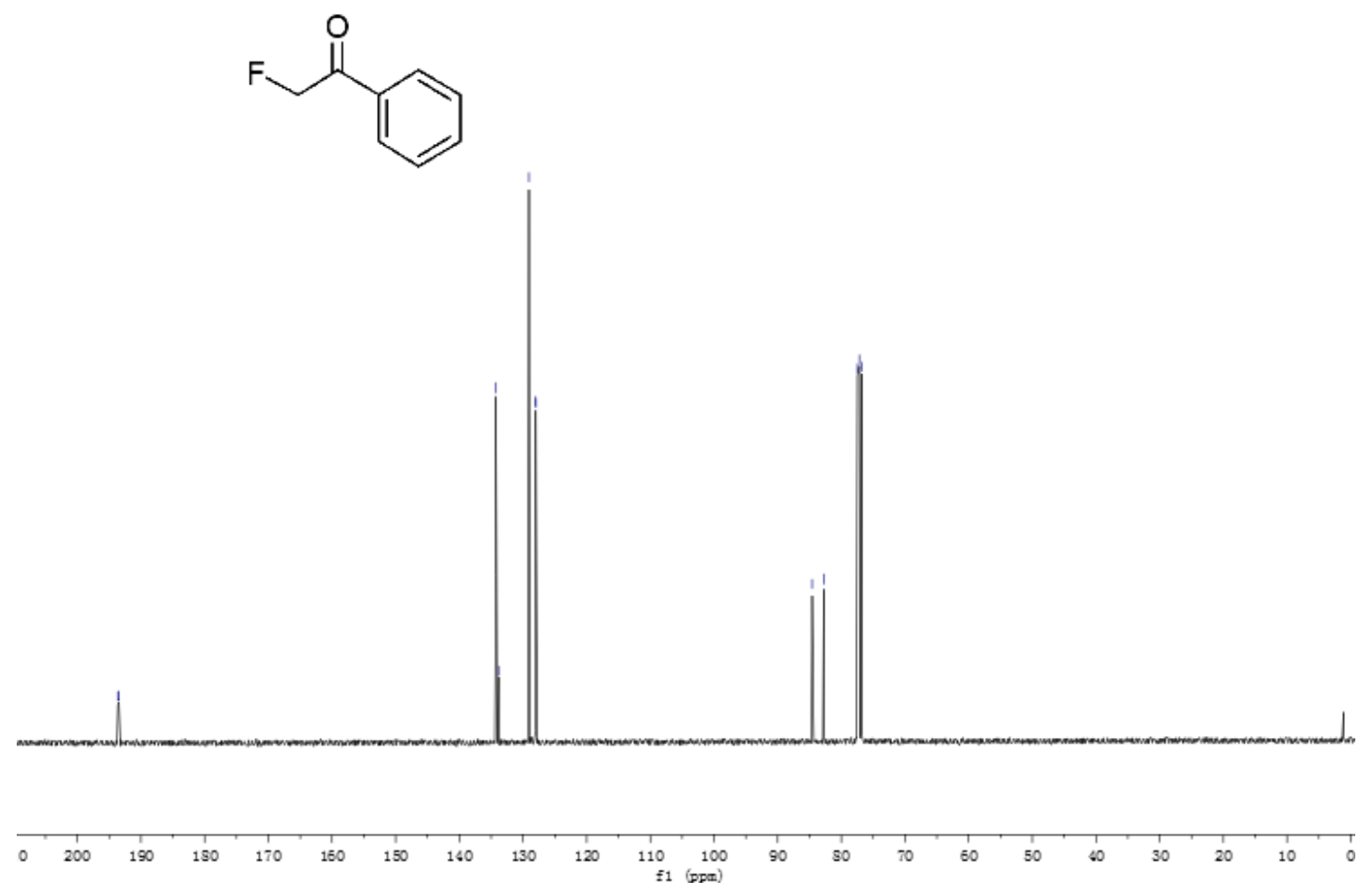

${ }^{19} \mathrm{~F}$ NMR of $\mathbf{2 b}$

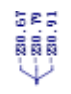
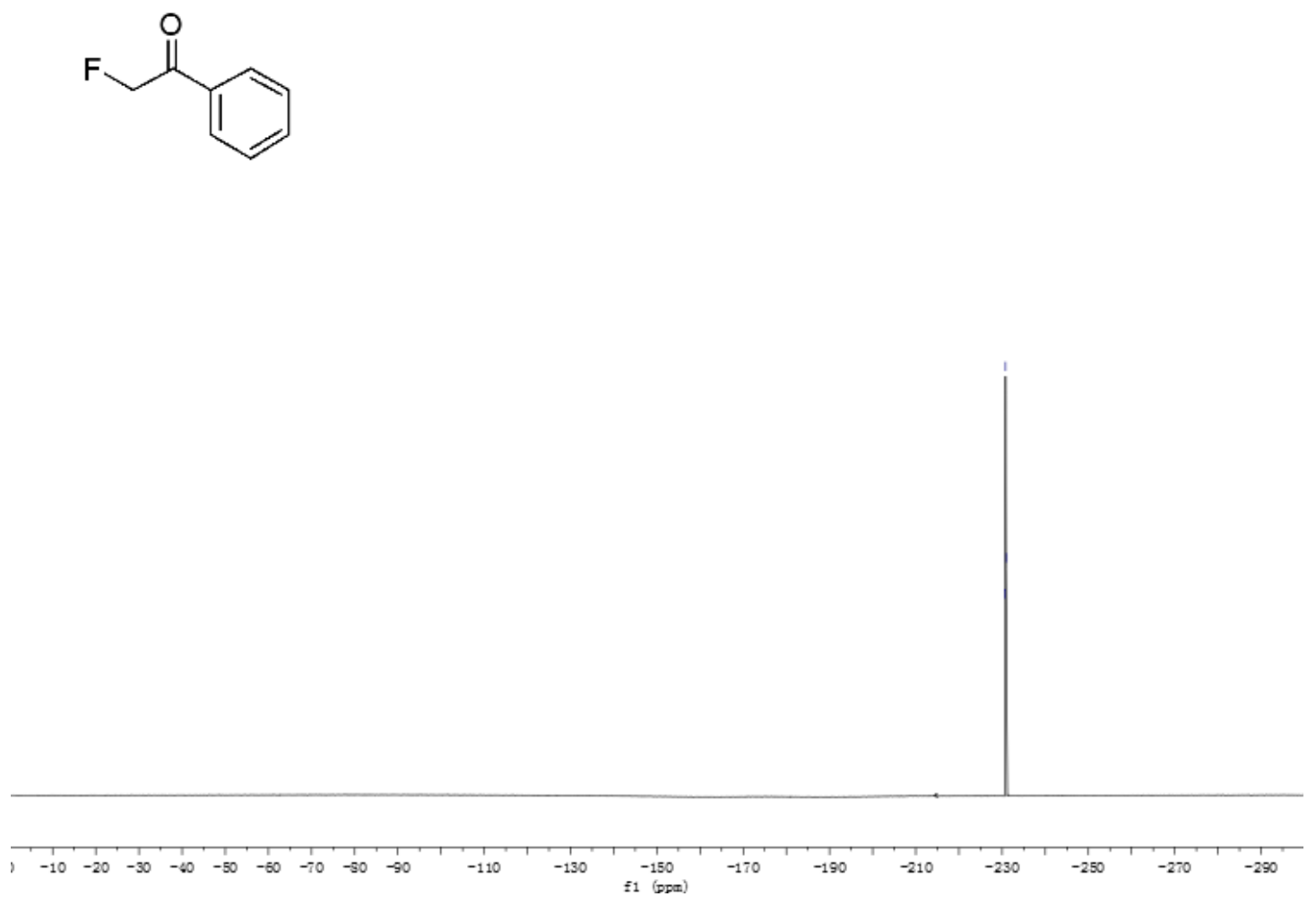

47 
${ }^{1} \mathrm{H}$ NMR of 2c
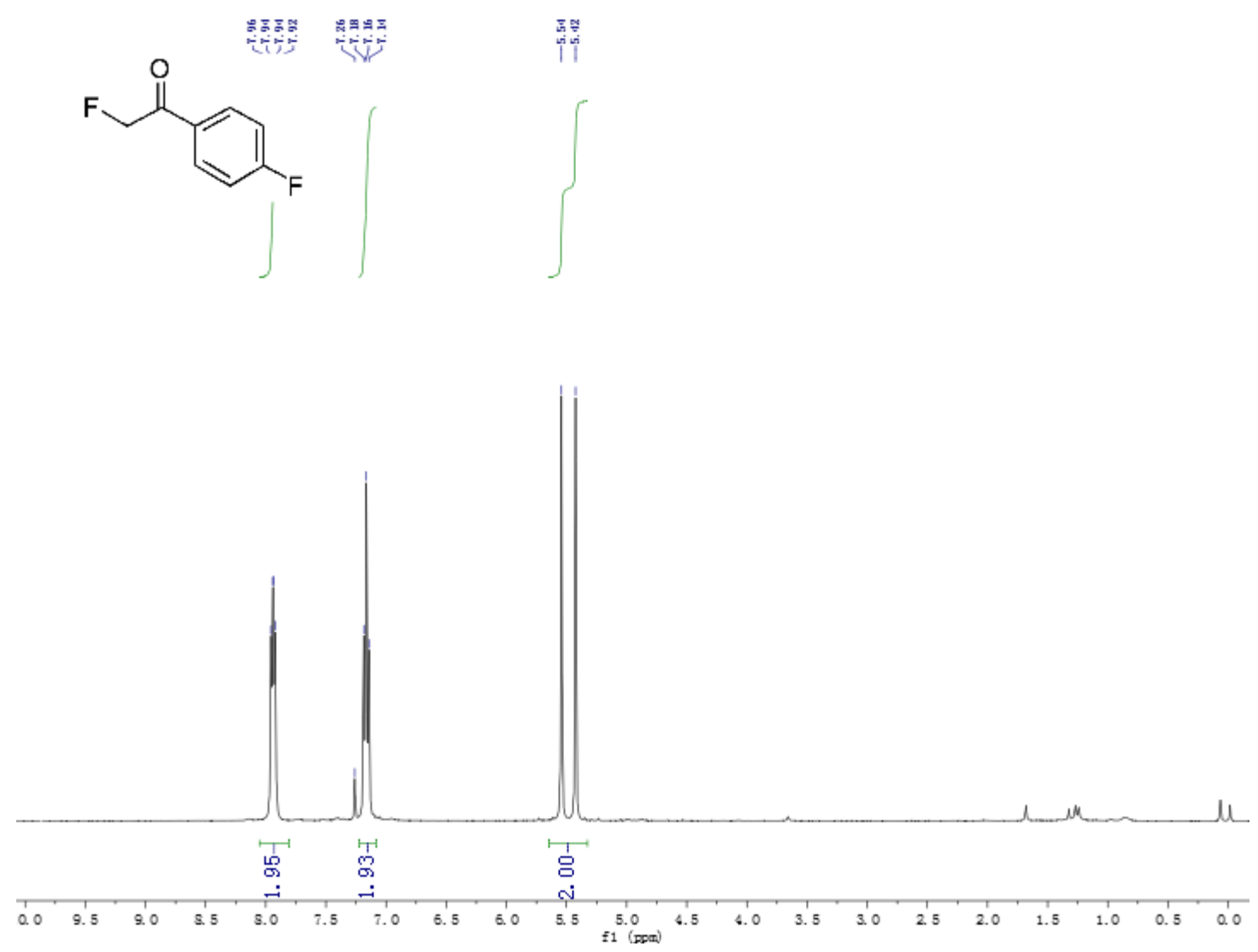

${ }^{13} \mathrm{C}$ NMR of 2c

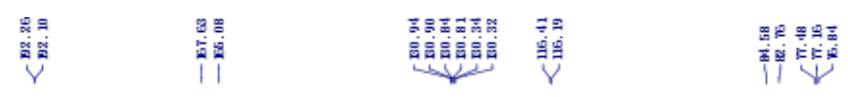<smiles>O=C(CF)c1ccc(F)cc1</smiles>

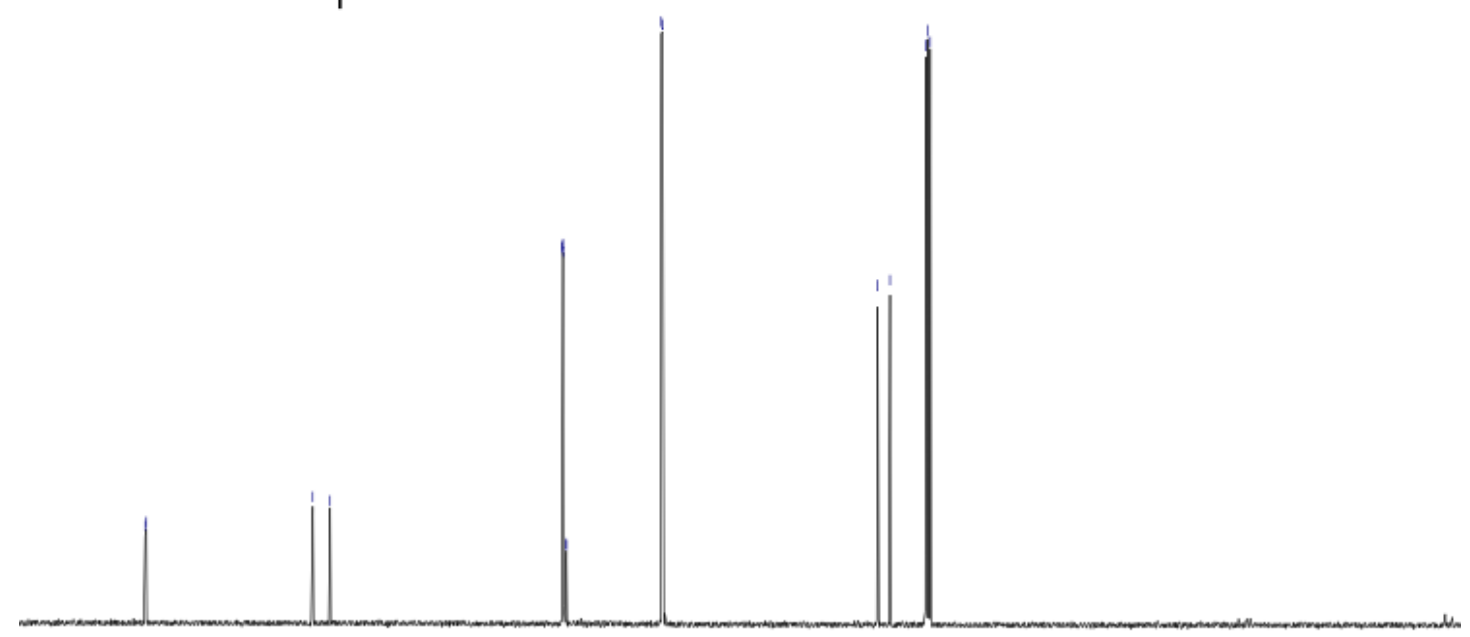

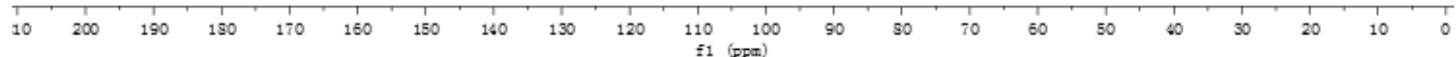


${ }^{19} \mathrm{~F}$ NMR of 2c<smiles>O=C(CF)c1ccc(F)cc1</smiles>

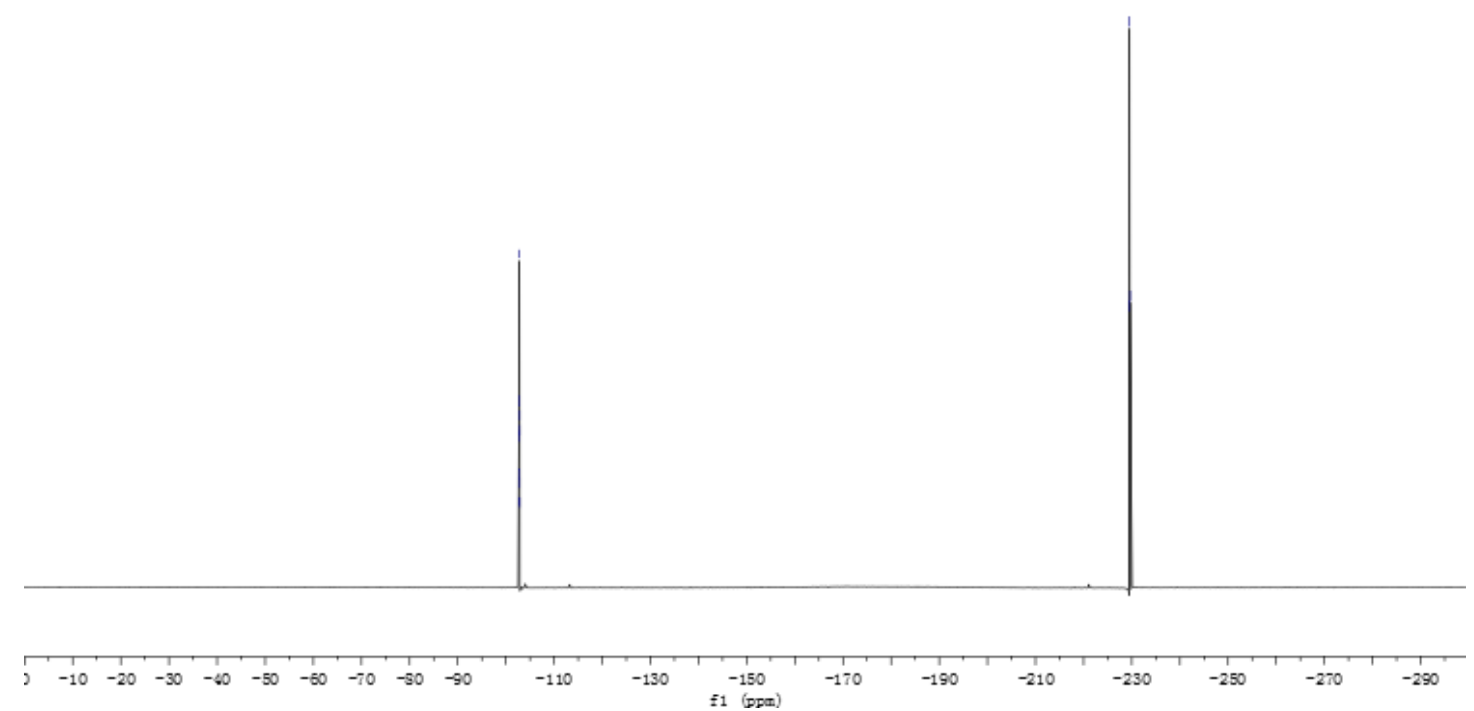

${ }^{1} \mathrm{H}$ NMR of 2d
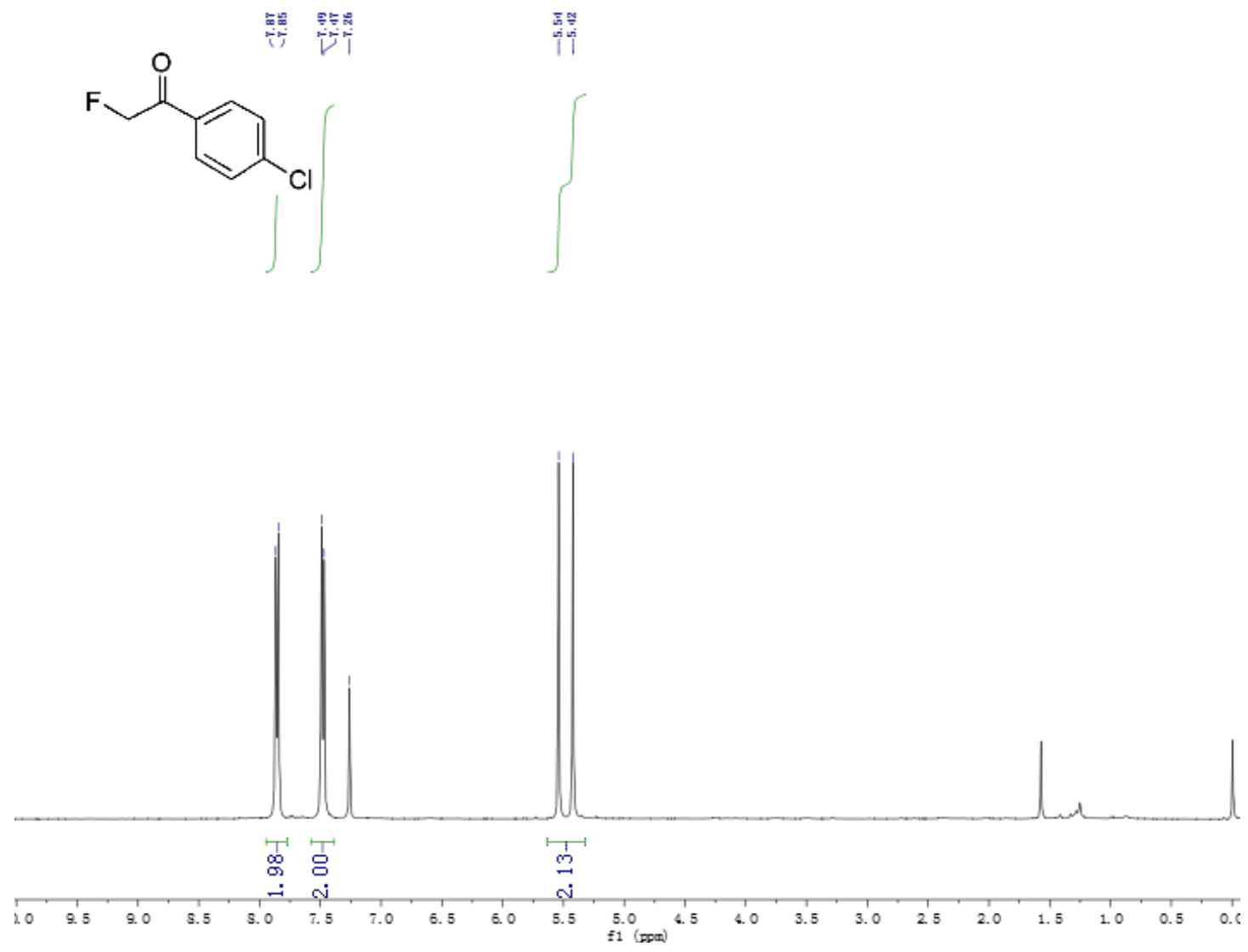
${ }^{13} \mathrm{C}$ NMR of ad

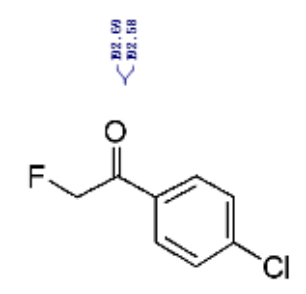

|

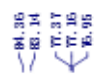

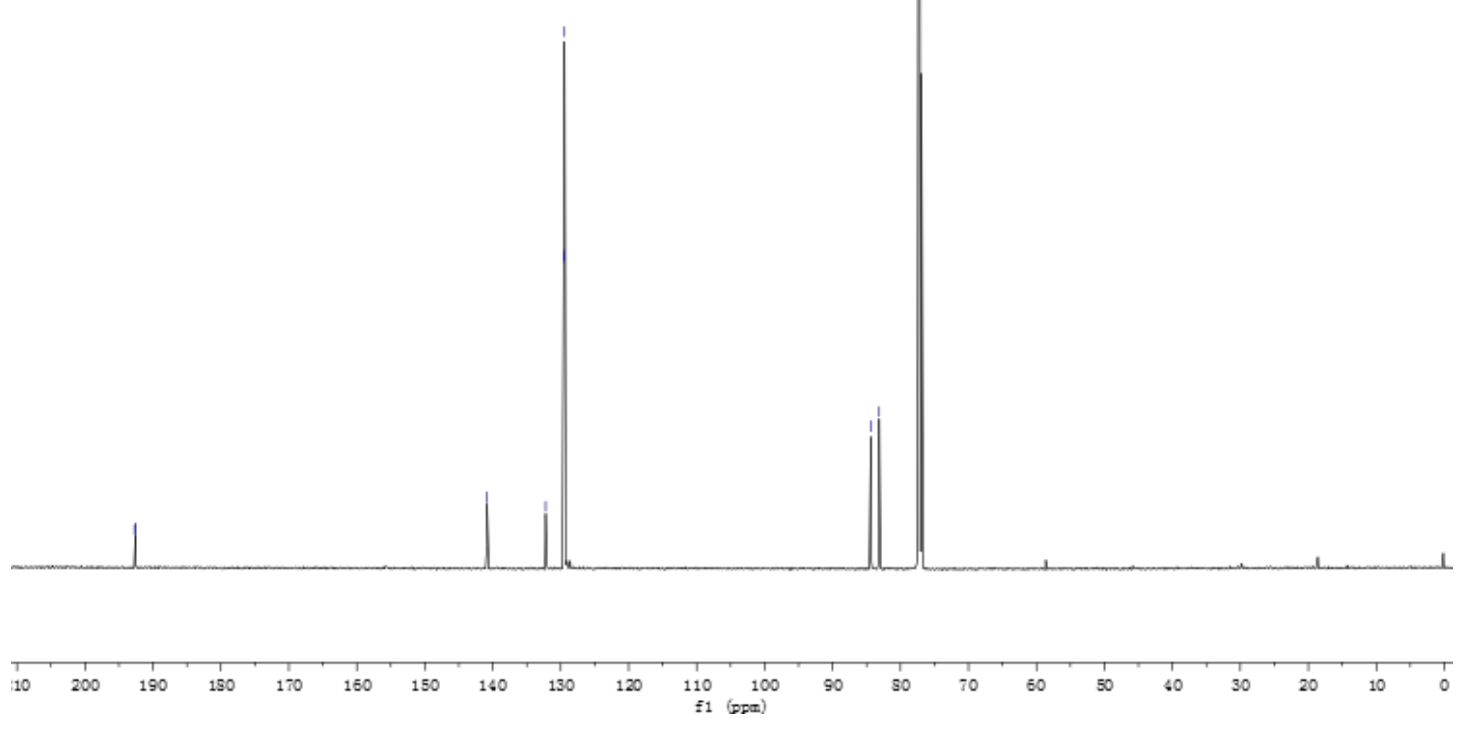

${ }^{19} \mathrm{~F}$ NMR of $2 \mathbf{d}$

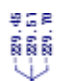
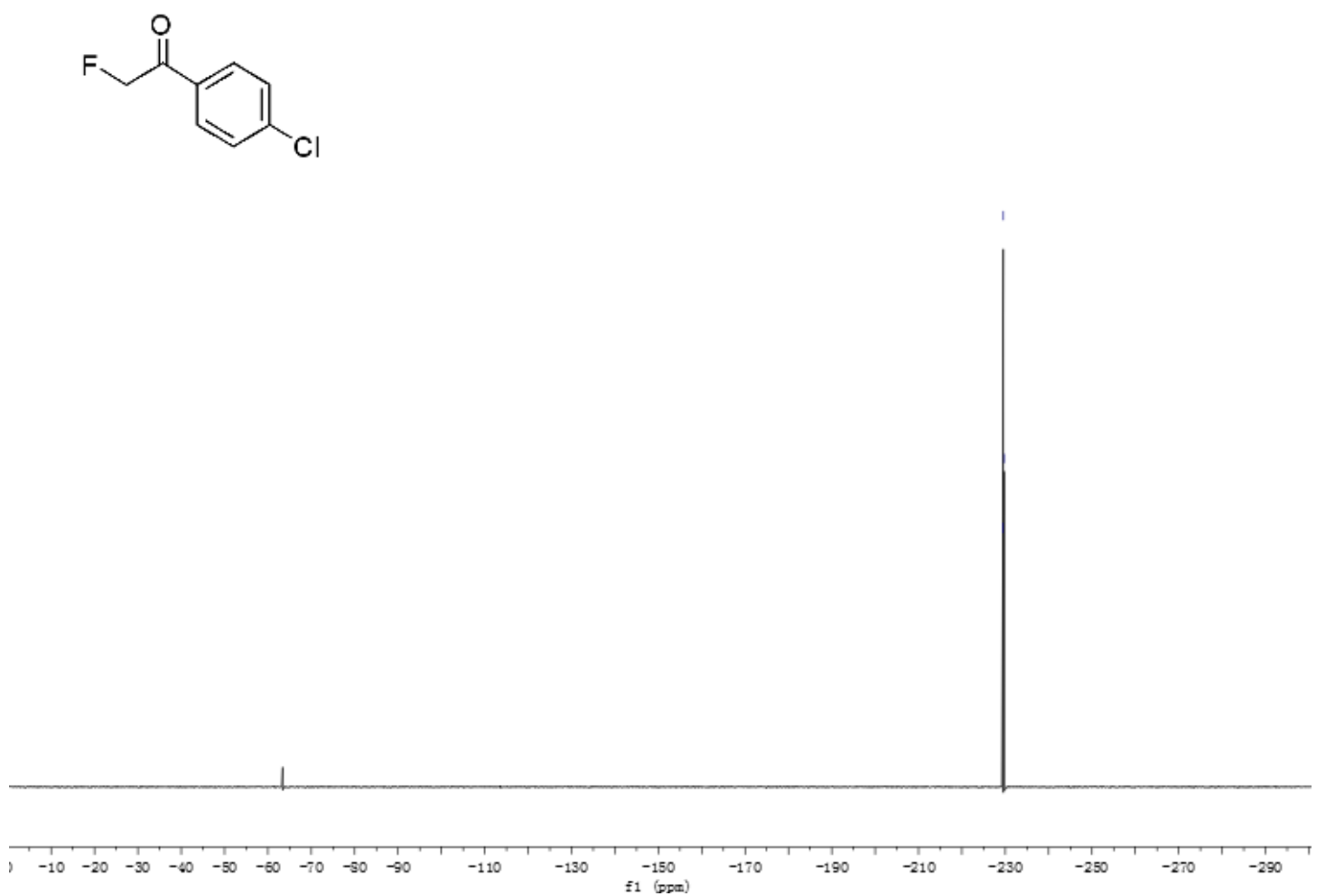

50 
${ }^{1} \mathrm{H}$ NMR of $\mathbf{2 e}$
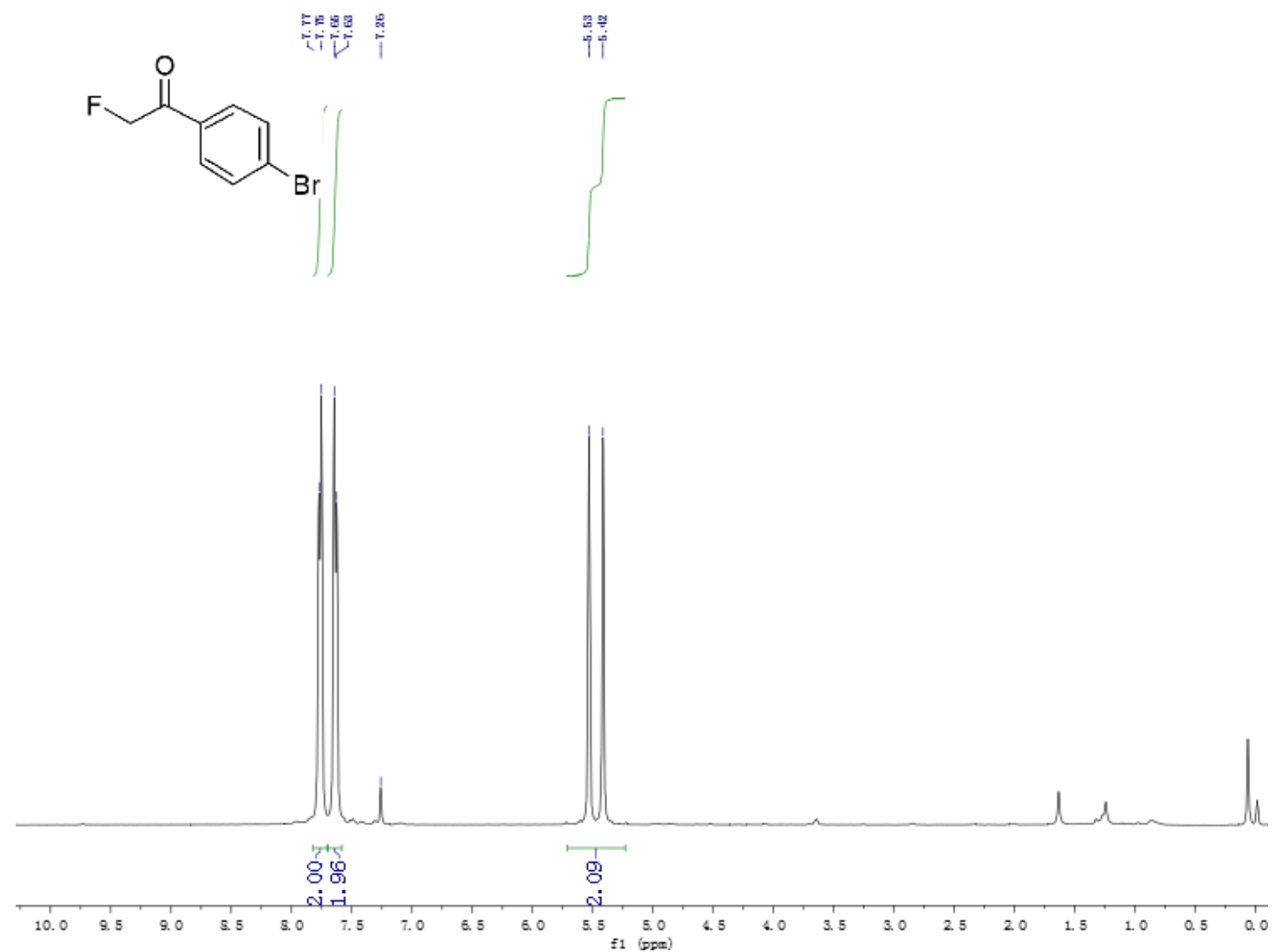

${ }^{13} \mathrm{C}$ NMR of $2 \mathbf{e}$

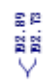

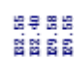

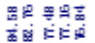

ํㅏㄴ

it<smiles>O=C(CF)c1ccc(Br)cc1</smiles>
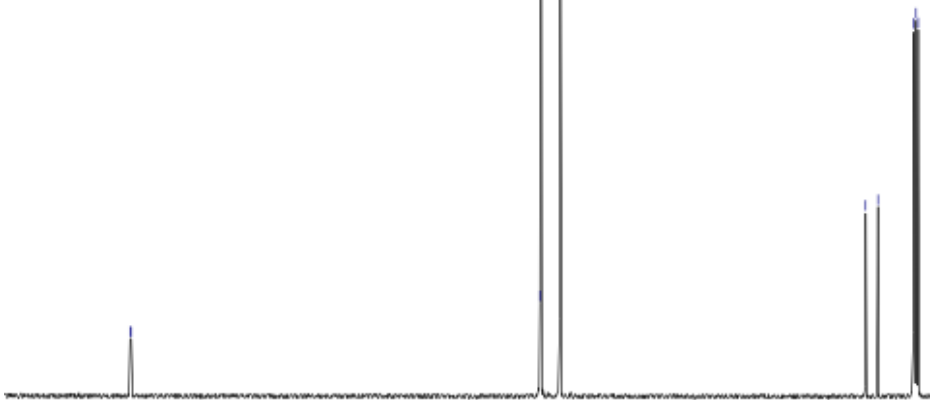

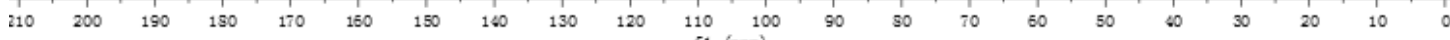


${ }^{19}$ F NMR of $2 \mathbf{e}$<smiles>O=C(CF)c1ccc(Br)cc1</smiles>

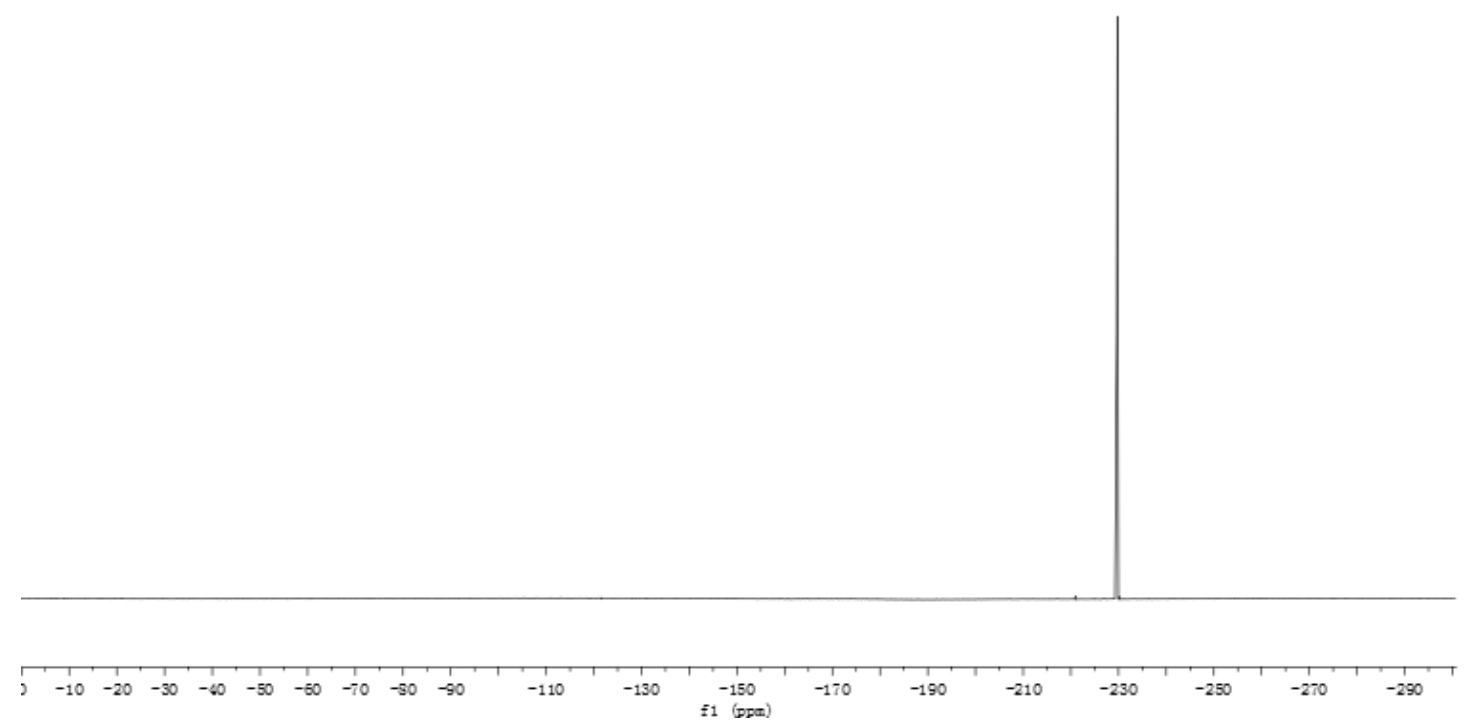

${ }^{1} \mathrm{H}$ NMR of $\mathbf{2 f}$

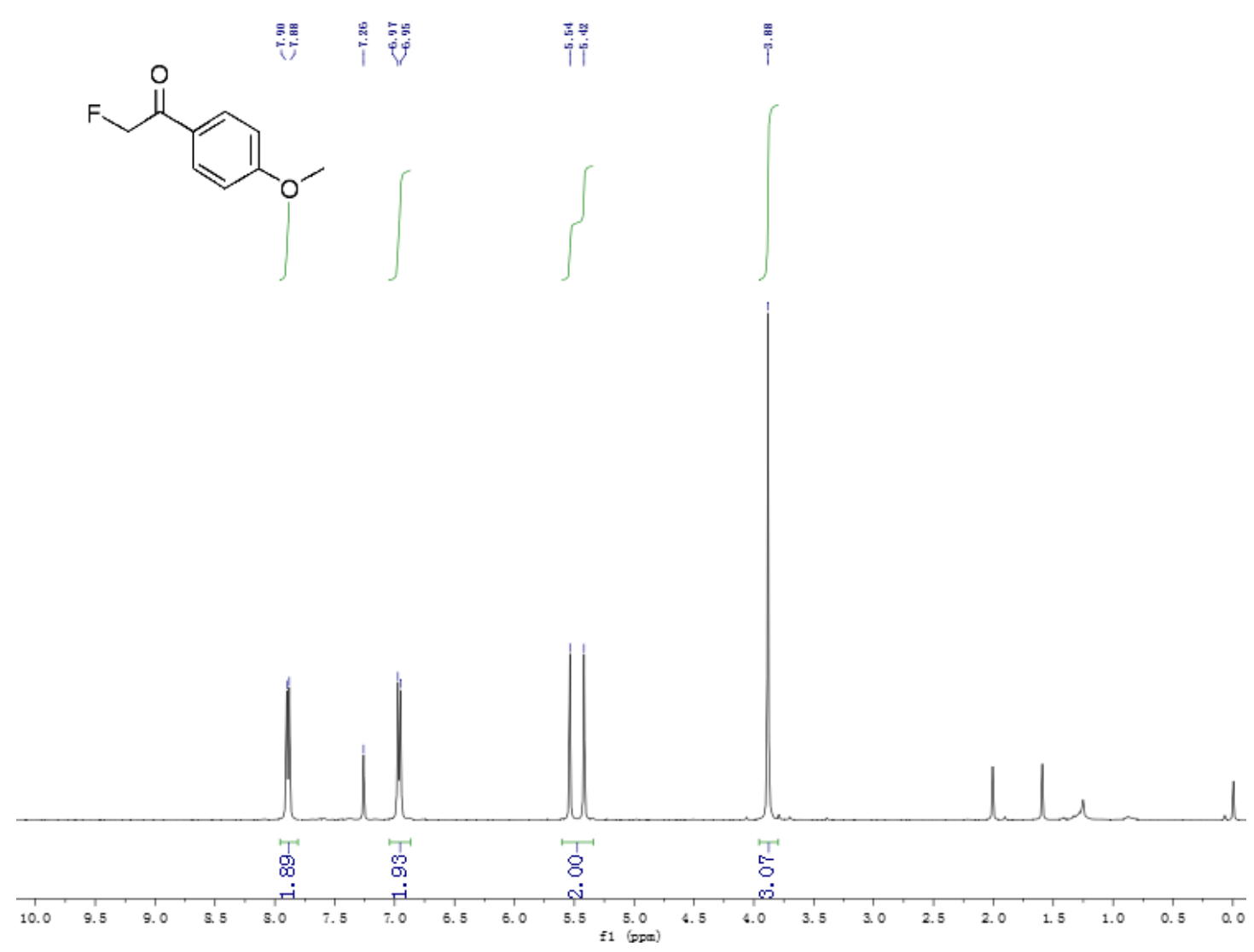


${ }^{13} \mathrm{C}$ NMR of $\mathbf{2 f}$

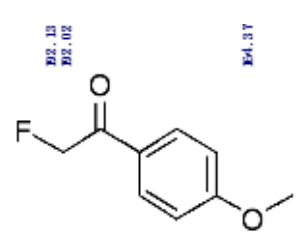

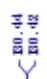

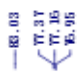

$\$$

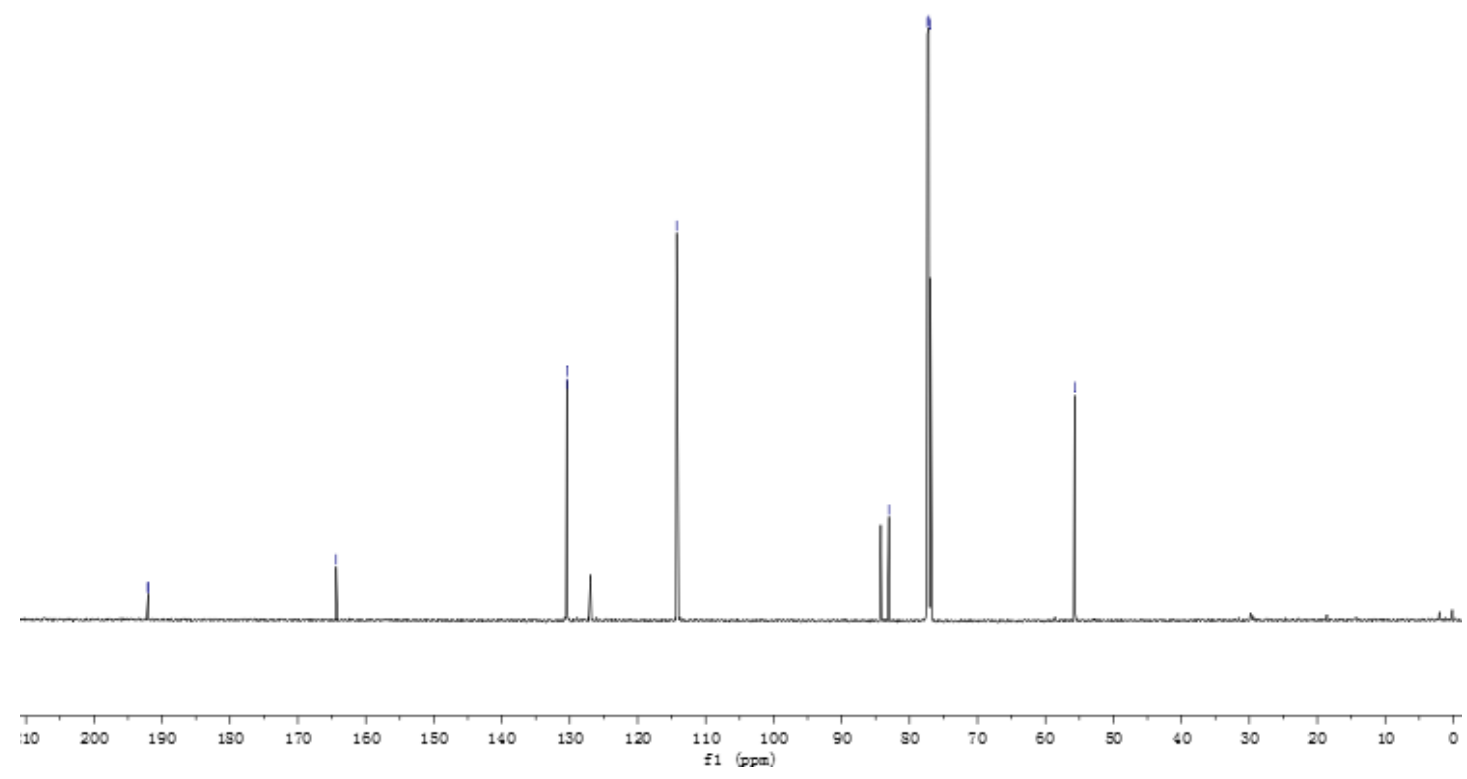

${ }^{19} \mathrm{~F}$ NMR of $\mathbf{2 f}$

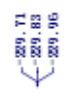

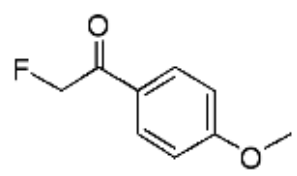

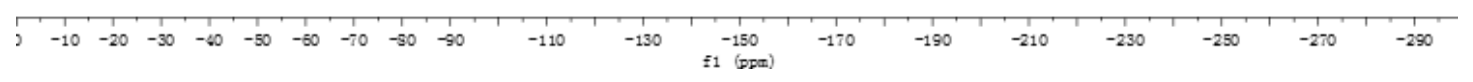

53 
${ }^{1} \mathrm{H}$ NMR of $\mathbf{2 g}$

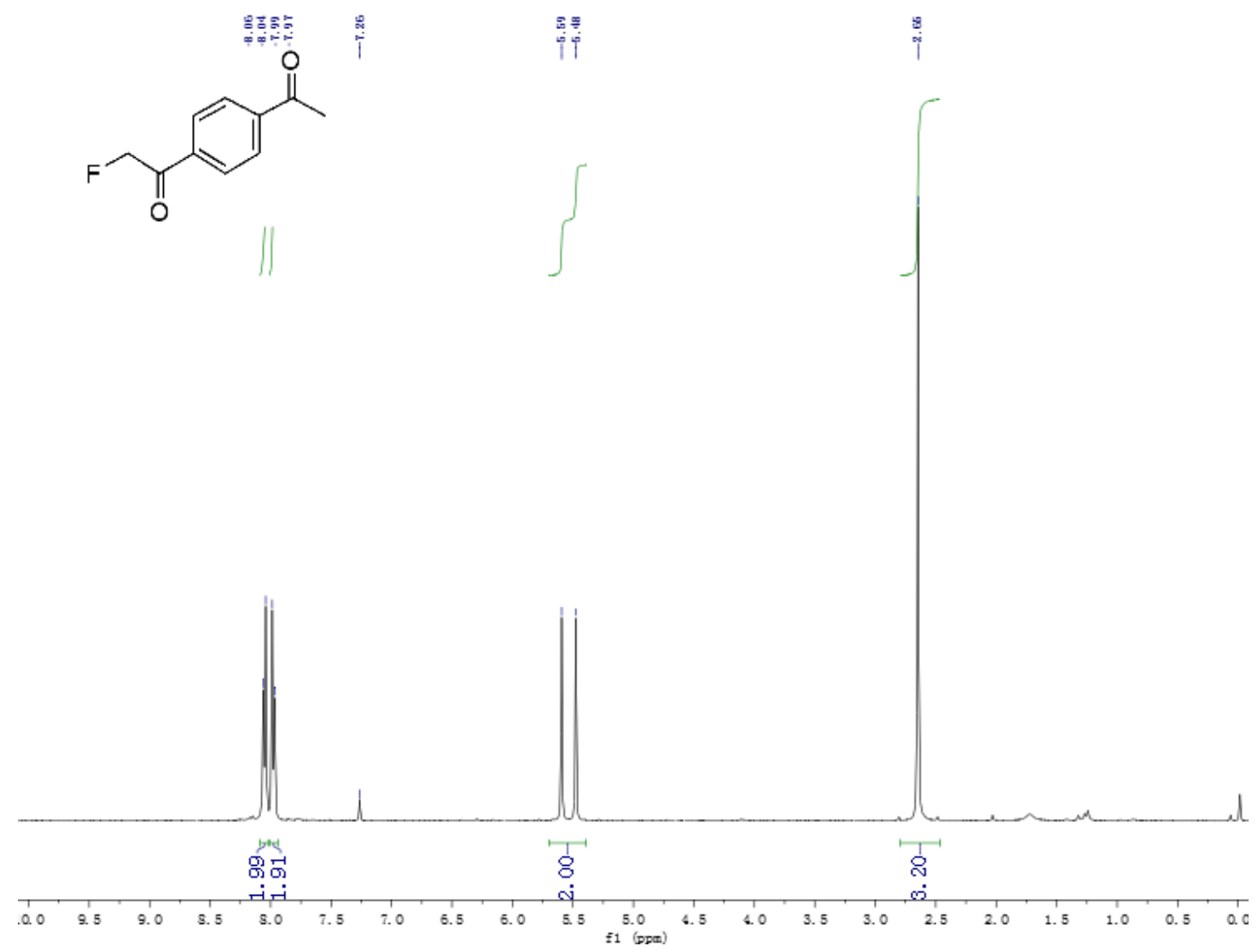

${ }^{13} \mathrm{C}$ NMR of $2 \mathrm{~g}$

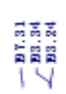

告

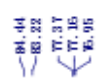

$\stackrel{5}{i}$<smiles>CC(=O)c1ccc(C(=O)CF)cc1</smiles>

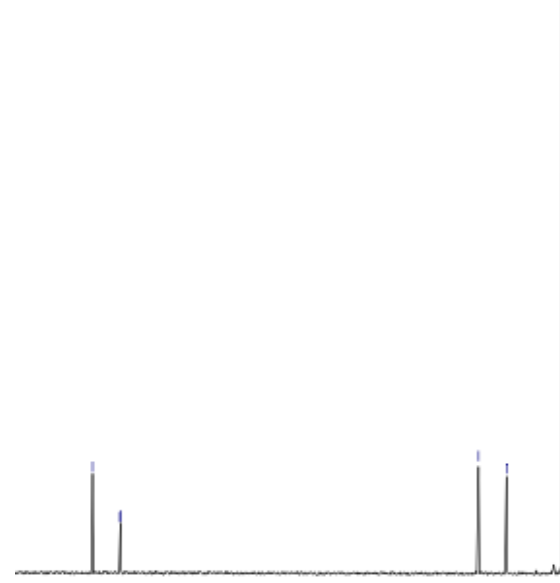

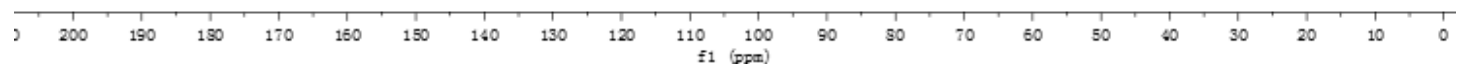


${ }^{19}$ F NMR of $2 \mathbf{g}$

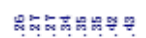

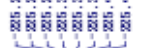<smiles>CC(=O)c1ccc(C(=O)CF)cc1</smiles>

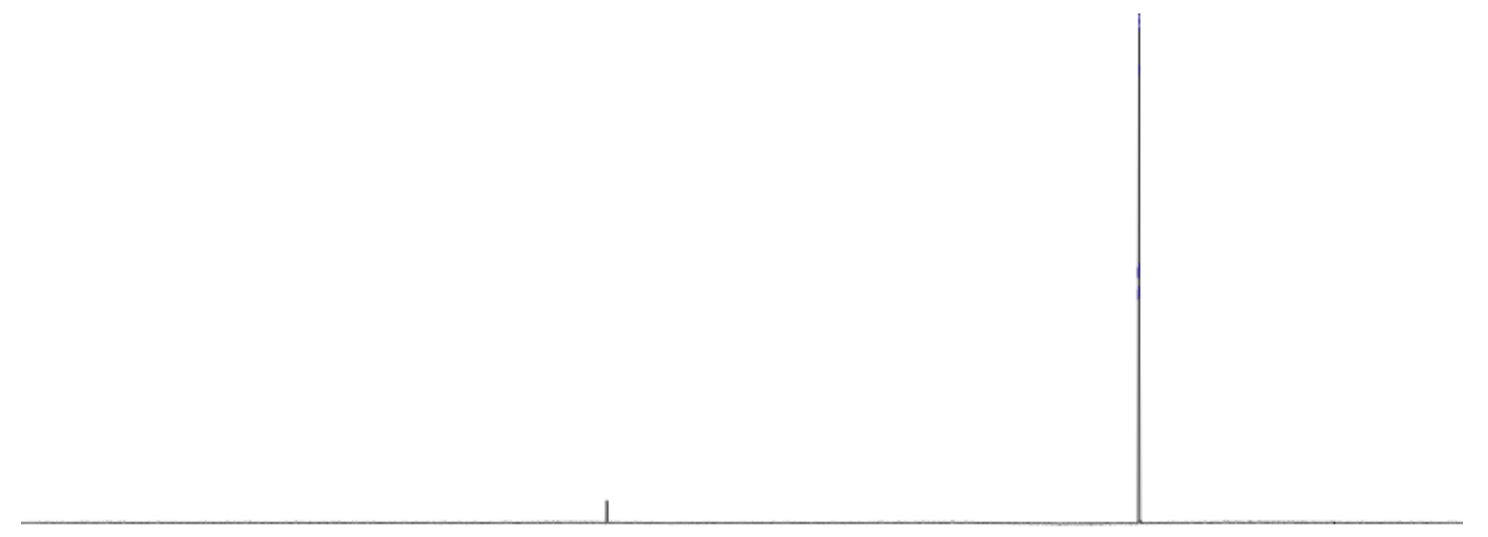

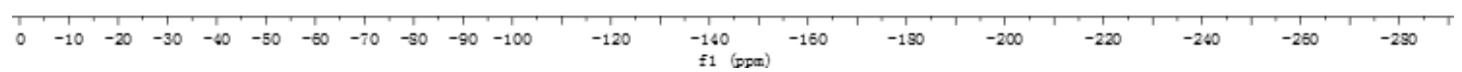

${ }^{1} \mathrm{H}$ NMR of $\mathbf{2 h}$

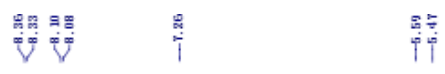<smiles>CCCCCCCCCCC(=O)c1ccc([N+](=O)[O-])cc1</smiles>

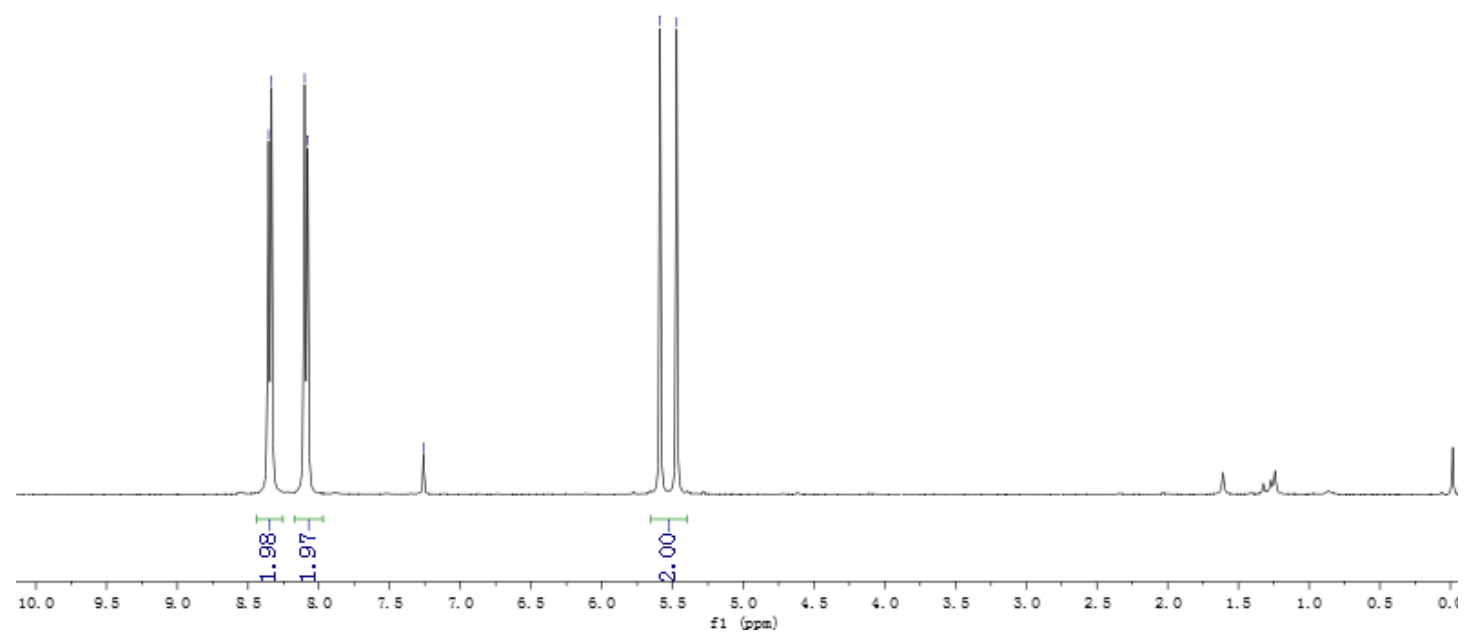


${ }^{13} \mathrm{C}$ NMR of $\mathbf{2 h}$

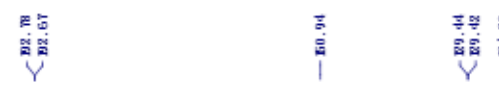

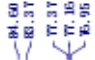

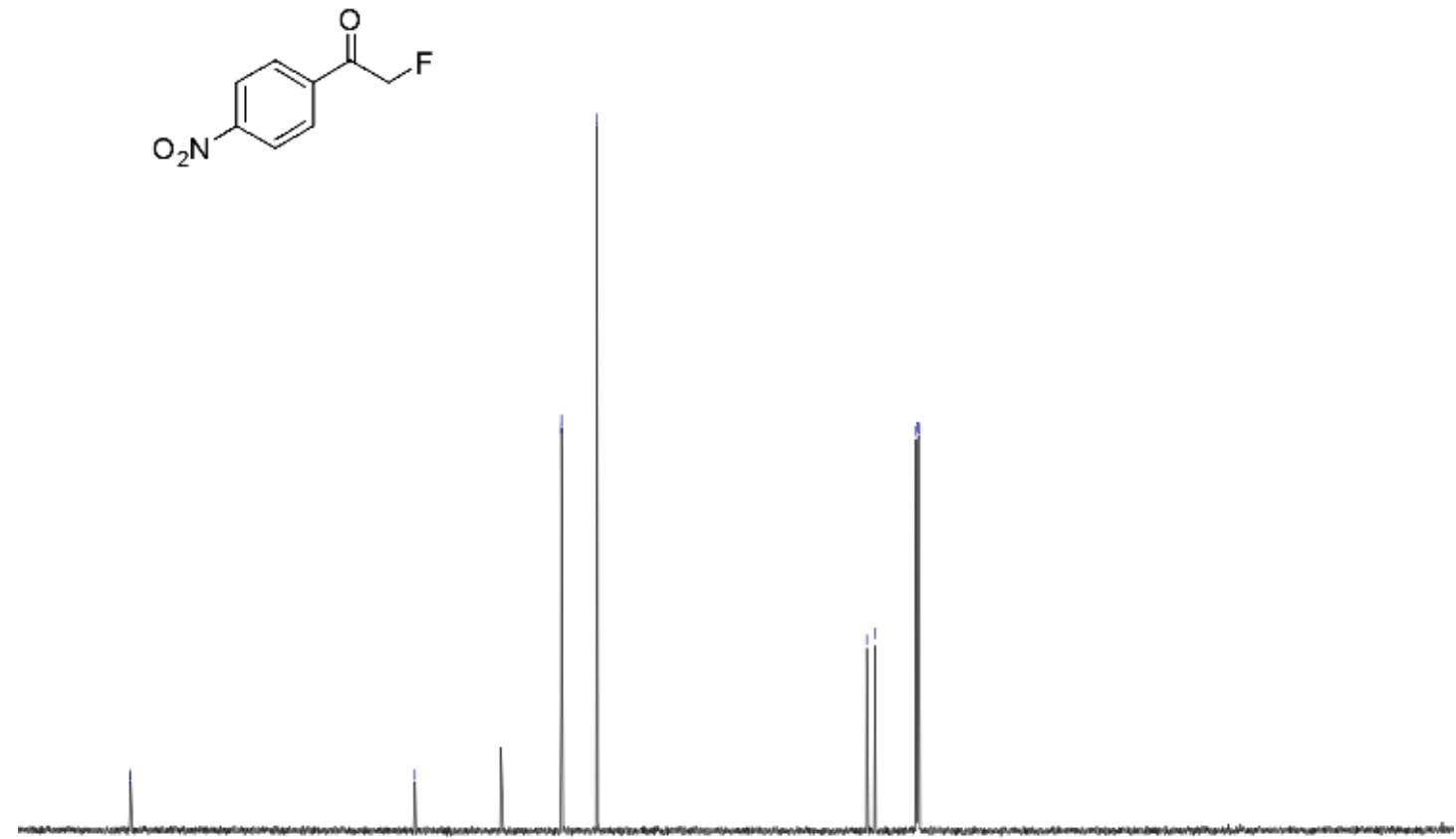

$\begin{array}{lllllllllllllllllllllllllllll}1 & 200 & 190 & 180 & 170 & 160 & 150 & 140 & 130 & 120 & 110 & 100 & 90 & 50 & 70 & 60 & 50 & 40 & 30 & 20 & 10 & 0\end{array}$

${ }^{19} \mathrm{~F}$ NMR of $\mathbf{2 h}$<smiles>O=C(CF)c1ccc([N+](=O)[O-])cc1</smiles>
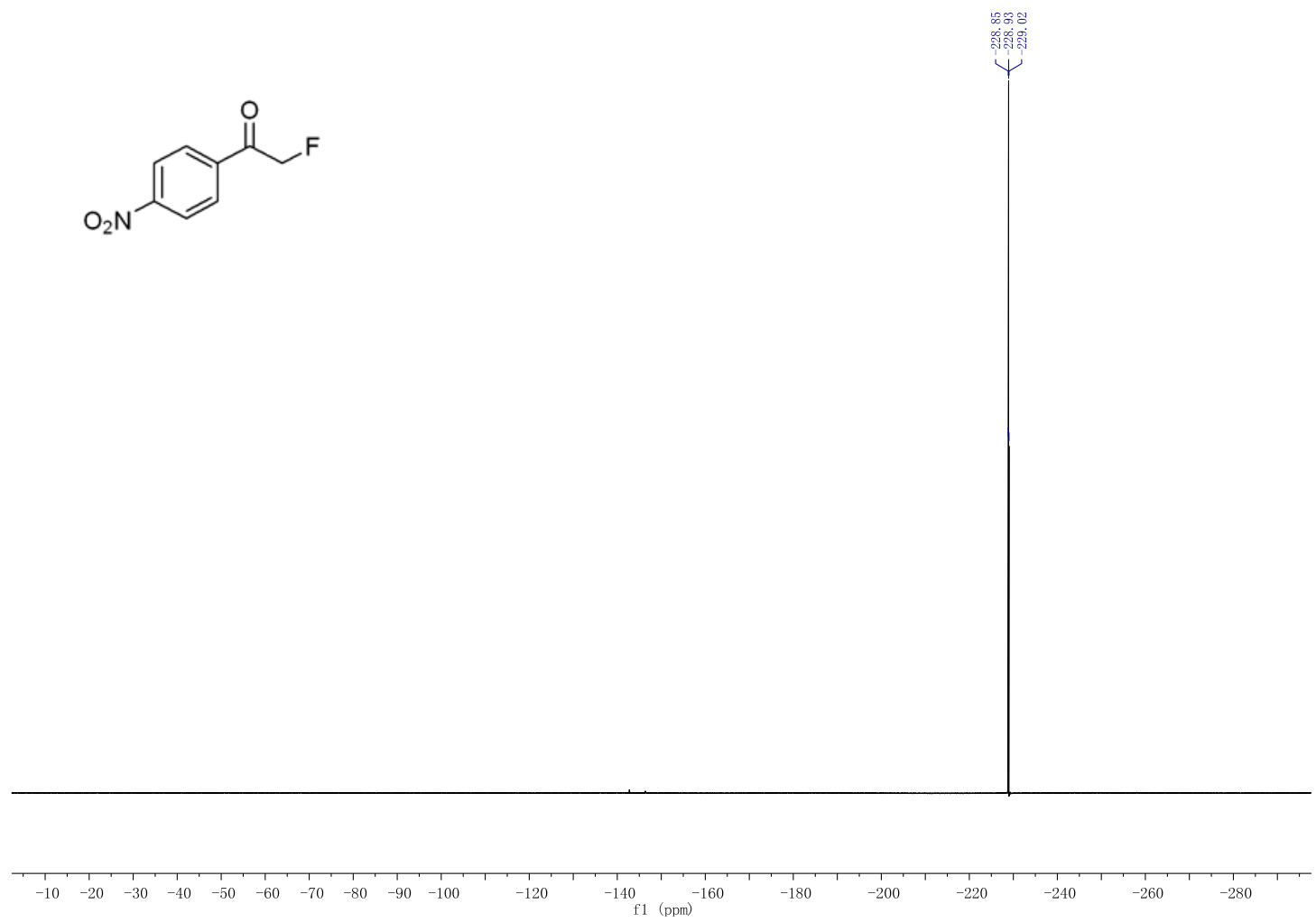
${ }^{1} \mathrm{H}$ NMR of $\mathbf{2 i}$

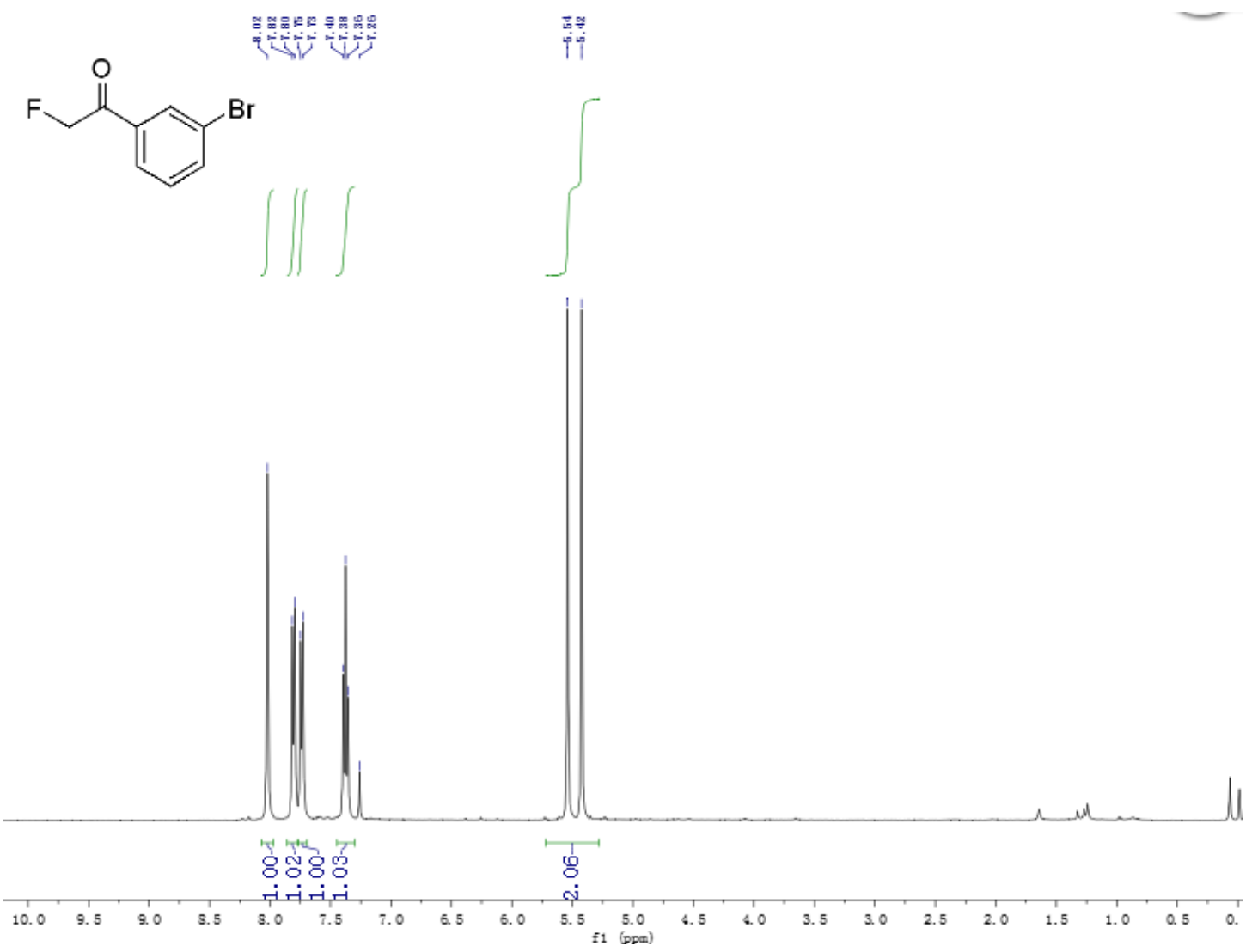

${ }^{13} \mathrm{C}$ NMR of $\mathbf{2 i}$
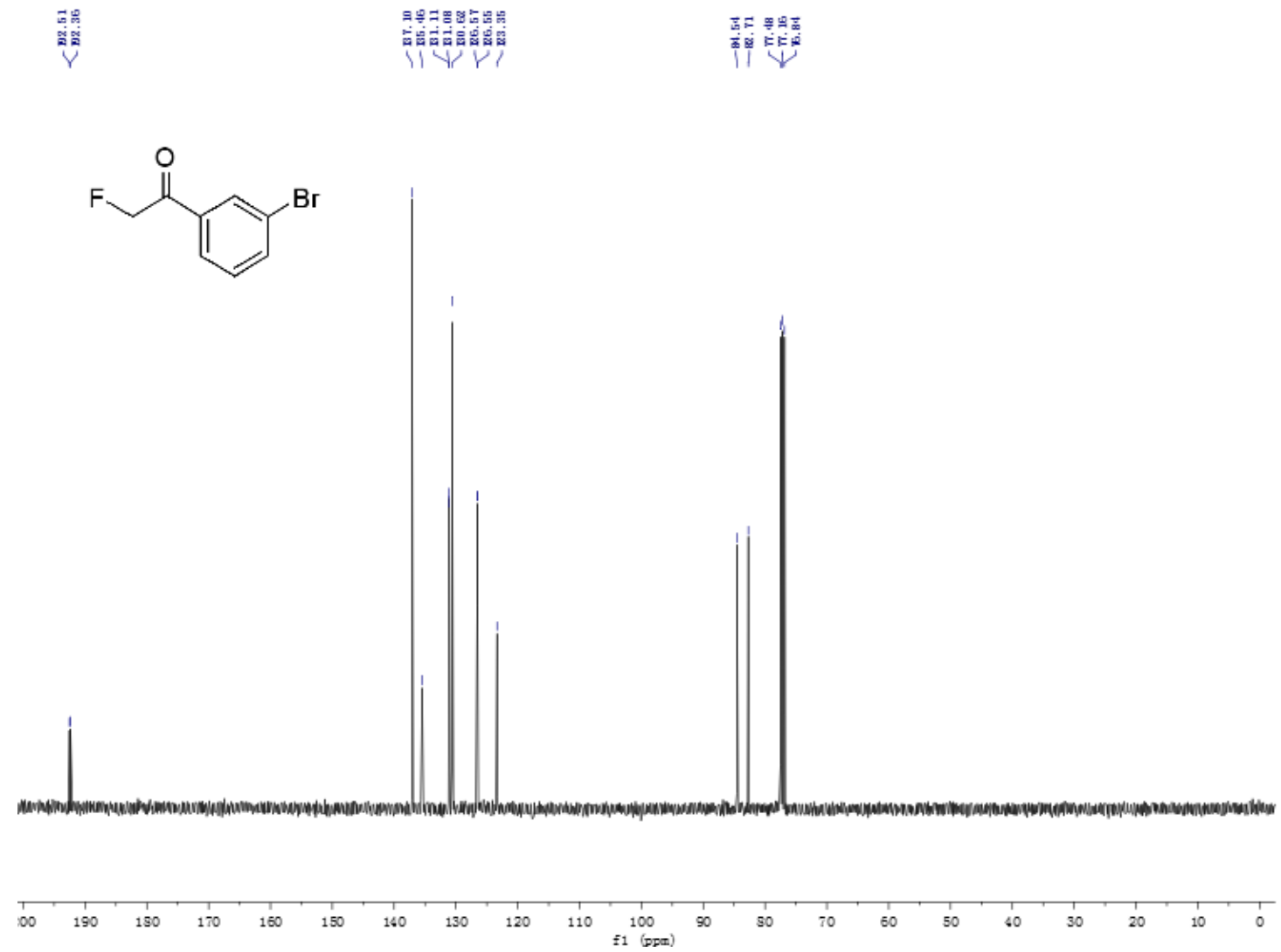
${ }^{19} \mathrm{~F}$ NMR of $\mathbf{2 i}$

怘象

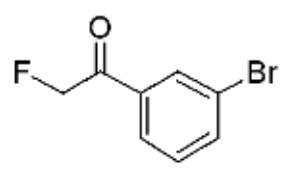

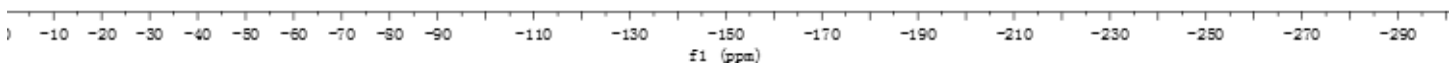

${ }^{1} \mathrm{H}$ NMR of $\mathbf{2} \mathbf{j}$

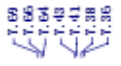

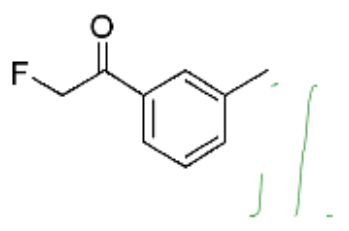

战

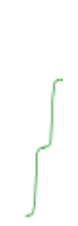

$\stackrel{?}{\text { ?ִ }}$

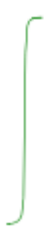

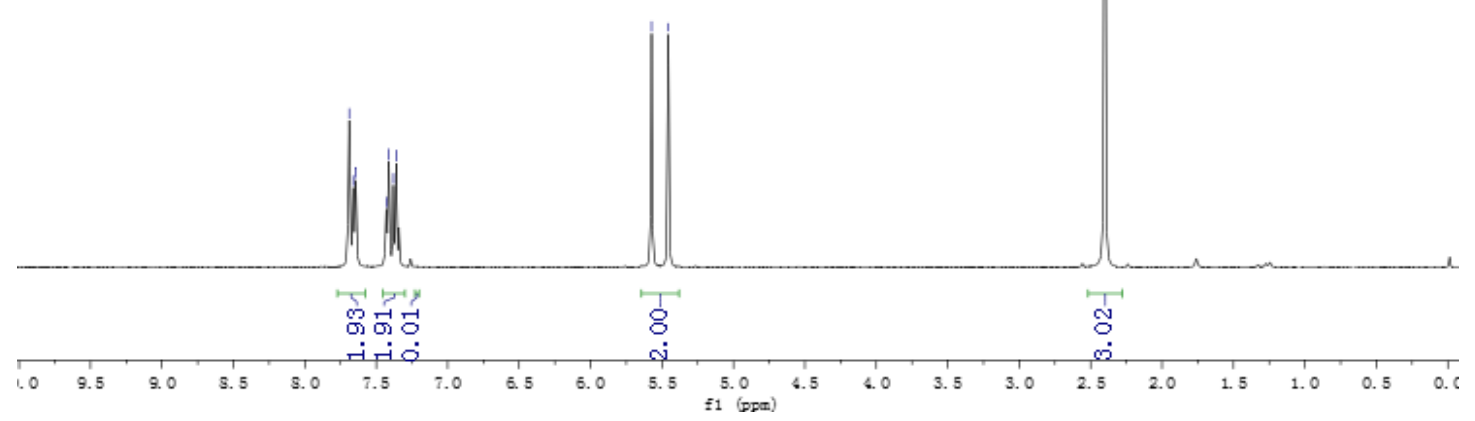

58 
${ }^{13} \mathrm{C}$ NMR of $\mathbf{2 j}$

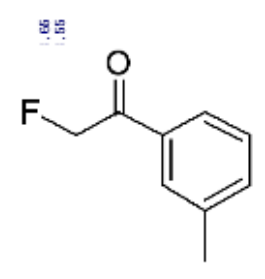

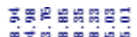

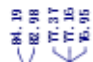

ํํำ
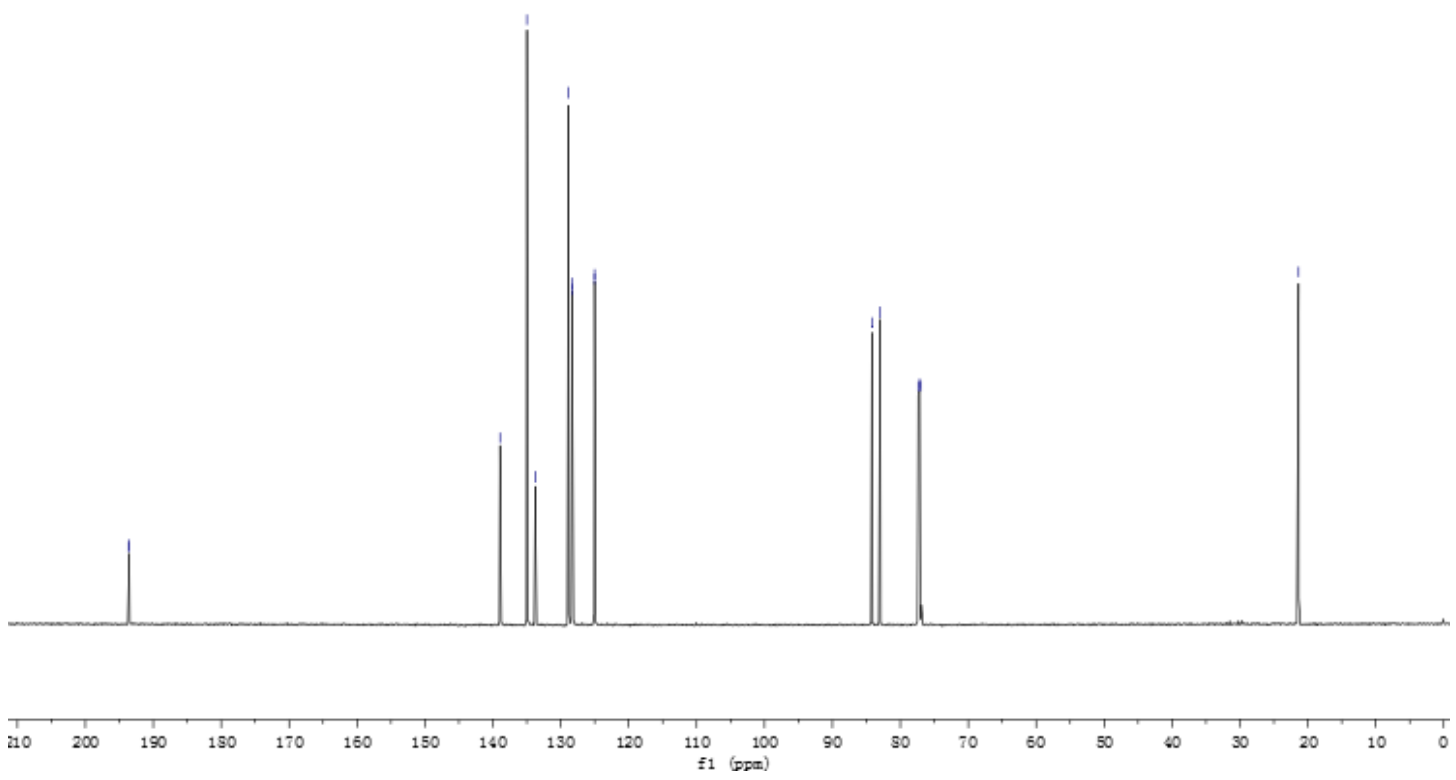

${ }^{19} \mathrm{~F}$ NMR of $\mathbf{2 j}$

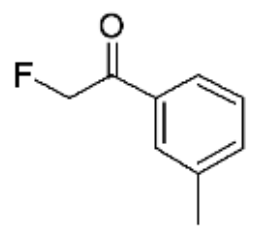

产
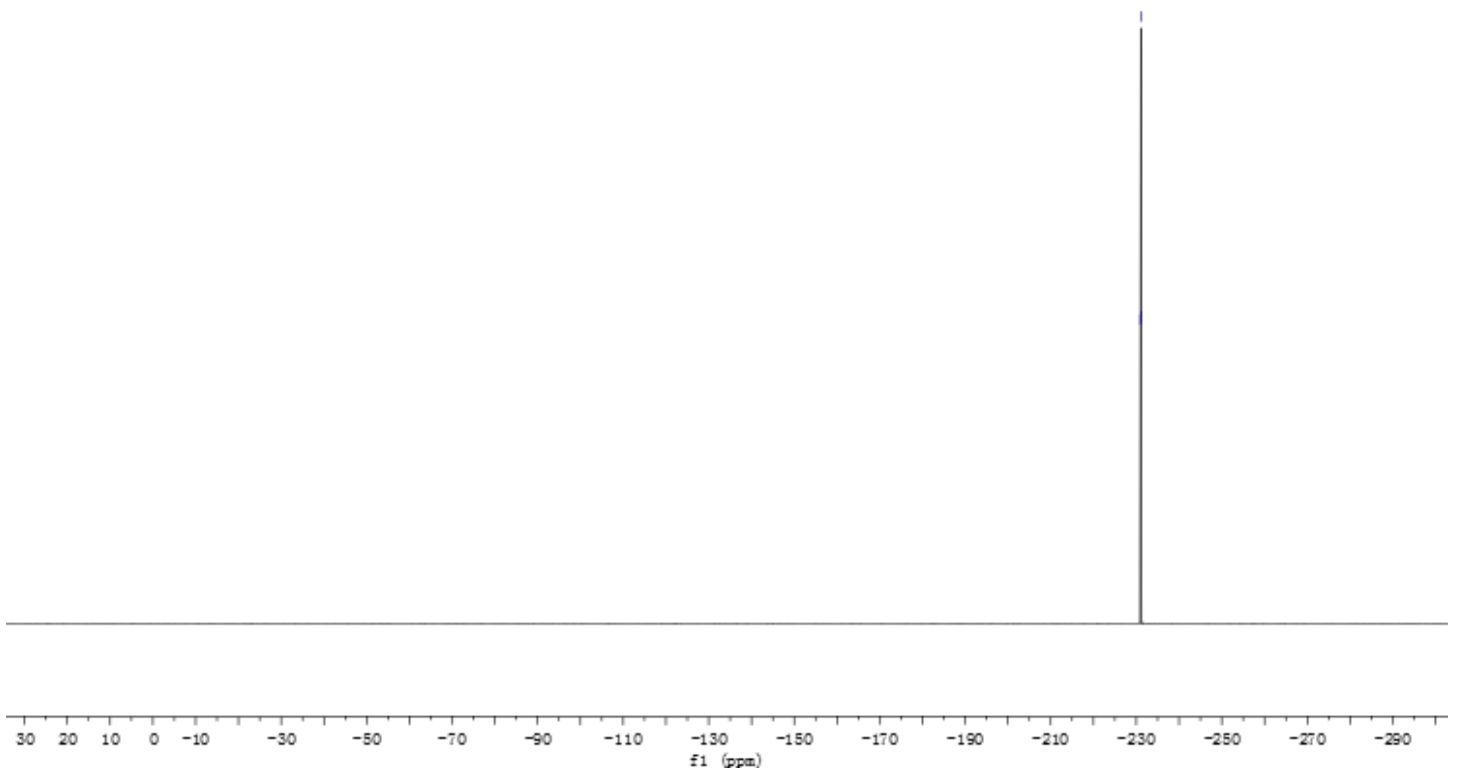

59 
${ }^{1} \mathrm{H}$ NMR of $\mathbf{2 k}$

98

?ִ<smiles>O=C(CF)c1ccccc1Br</smiles>

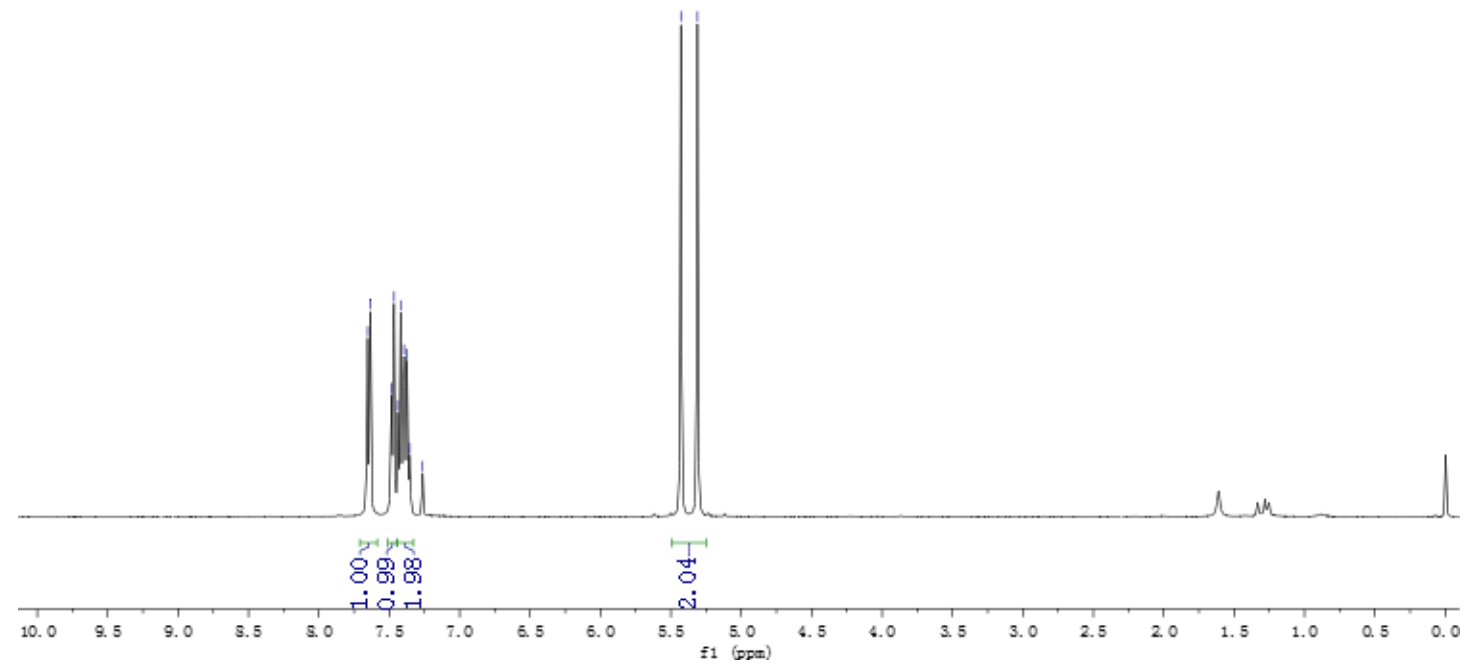

${ }^{13} \mathrm{C}$ NMR of $\mathbf{2 k}$

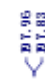

gyo

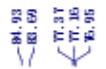<smiles>O=C(CF)c1ccccc1Br</smiles>

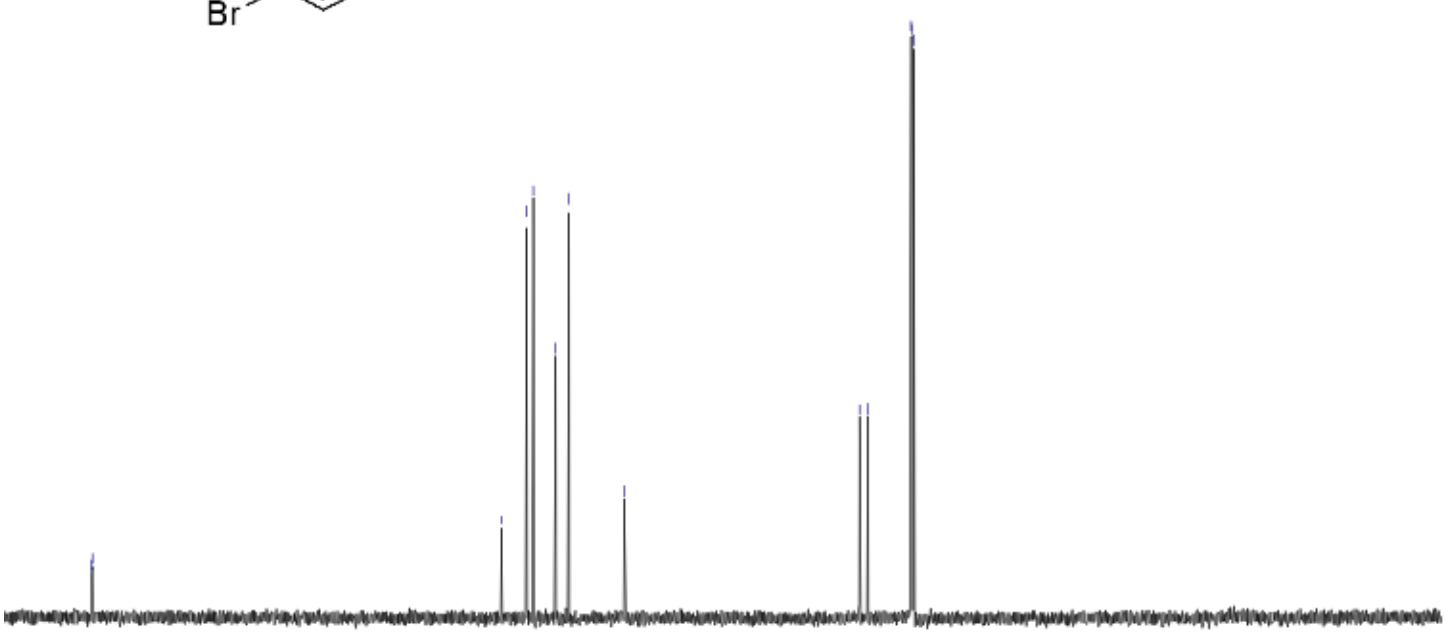

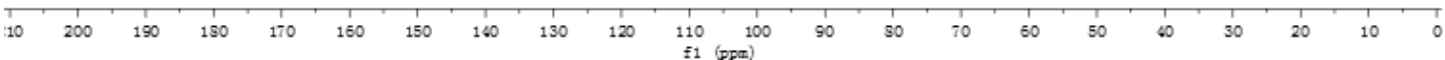


${ }^{19} \mathrm{~F}$ NMR of $\mathbf{2 k}$

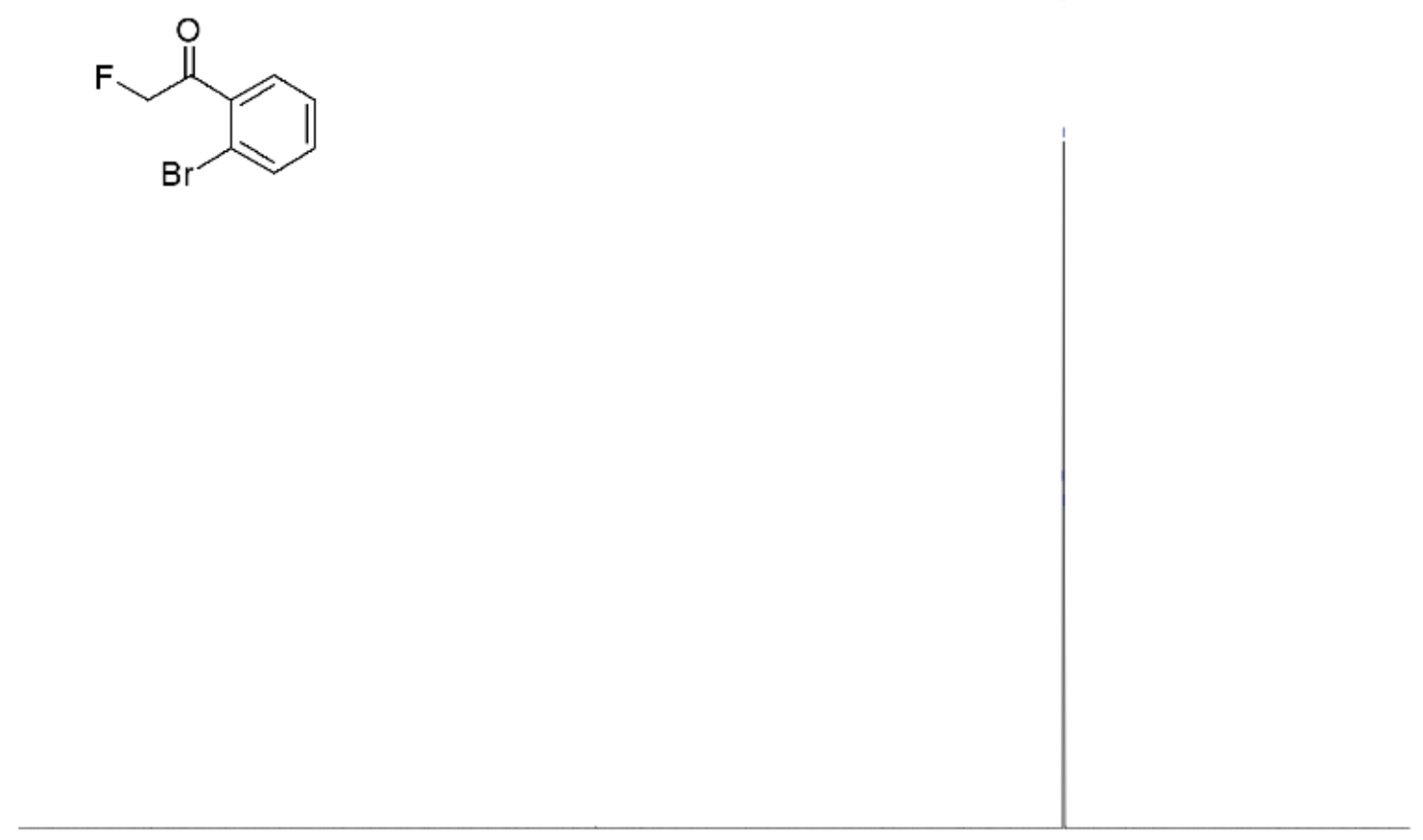

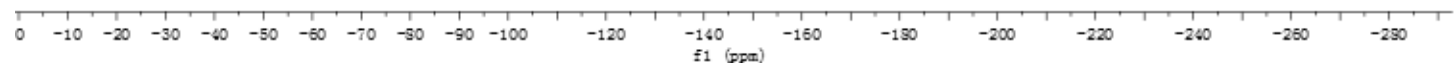

${ }^{1} \mathrm{H}$ NMR of $2 \mathbf{l}$
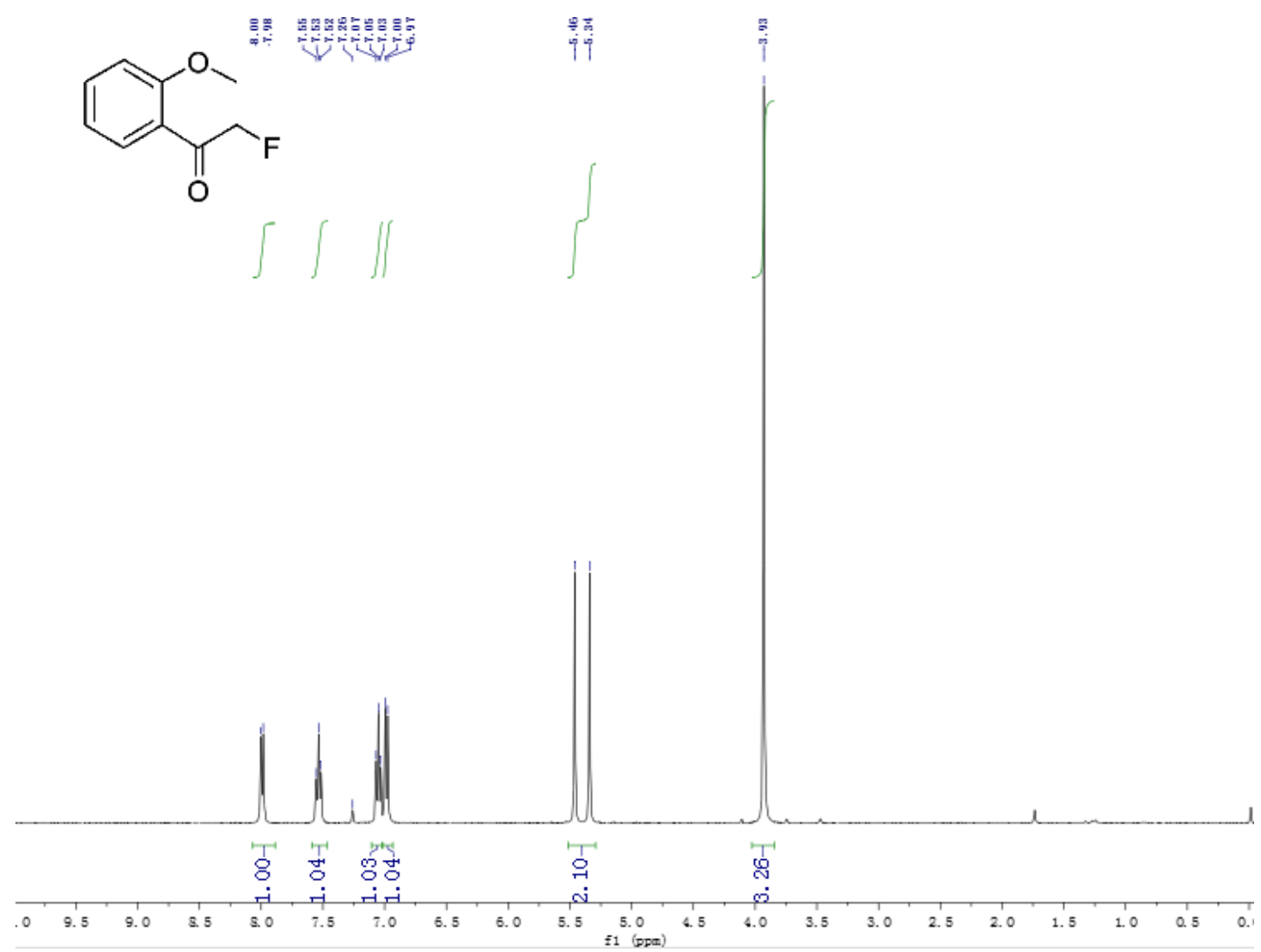
${ }^{13} \mathrm{C}$ NMR of 2 l
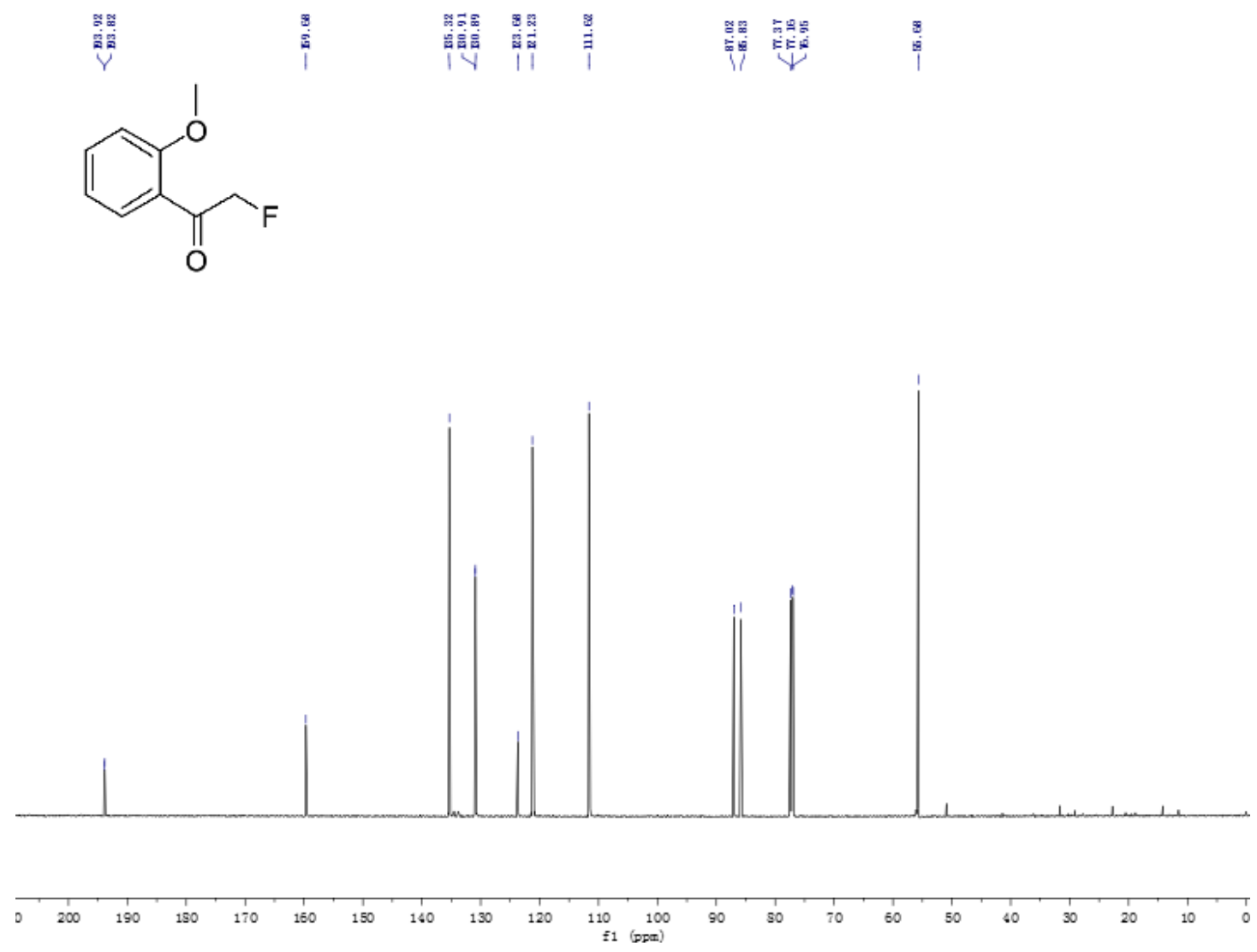

${ }^{19} \mathrm{~F}$ NMR of $2 \mathbf{l}$<smiles>COc1ccccc1C(=O)CF</smiles> 
${ }^{1} \mathrm{H}$ NMR of $\mathbf{2 m}$

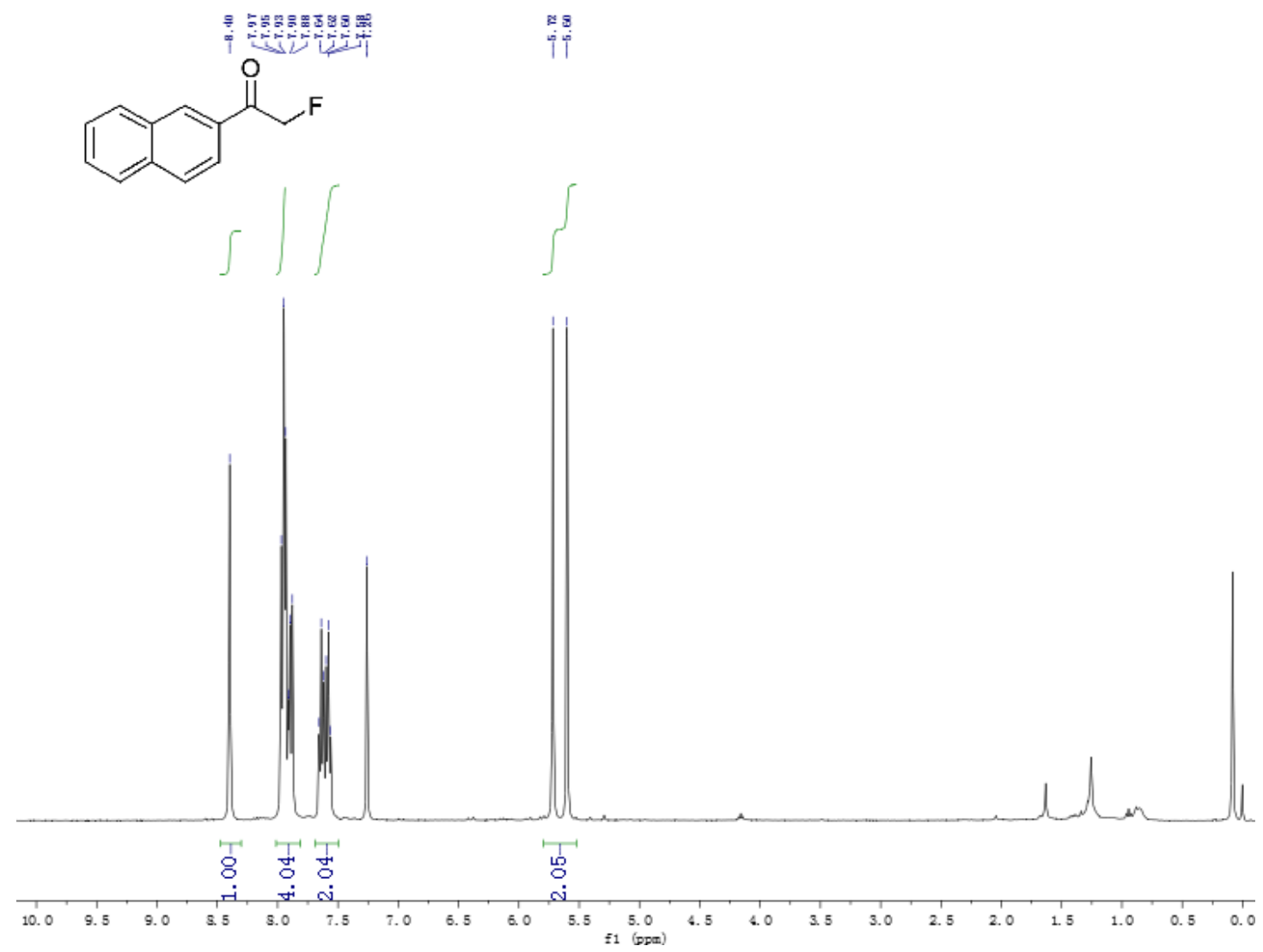

${ }^{13} \mathrm{C}$ NMR of $\mathbf{2 m}$
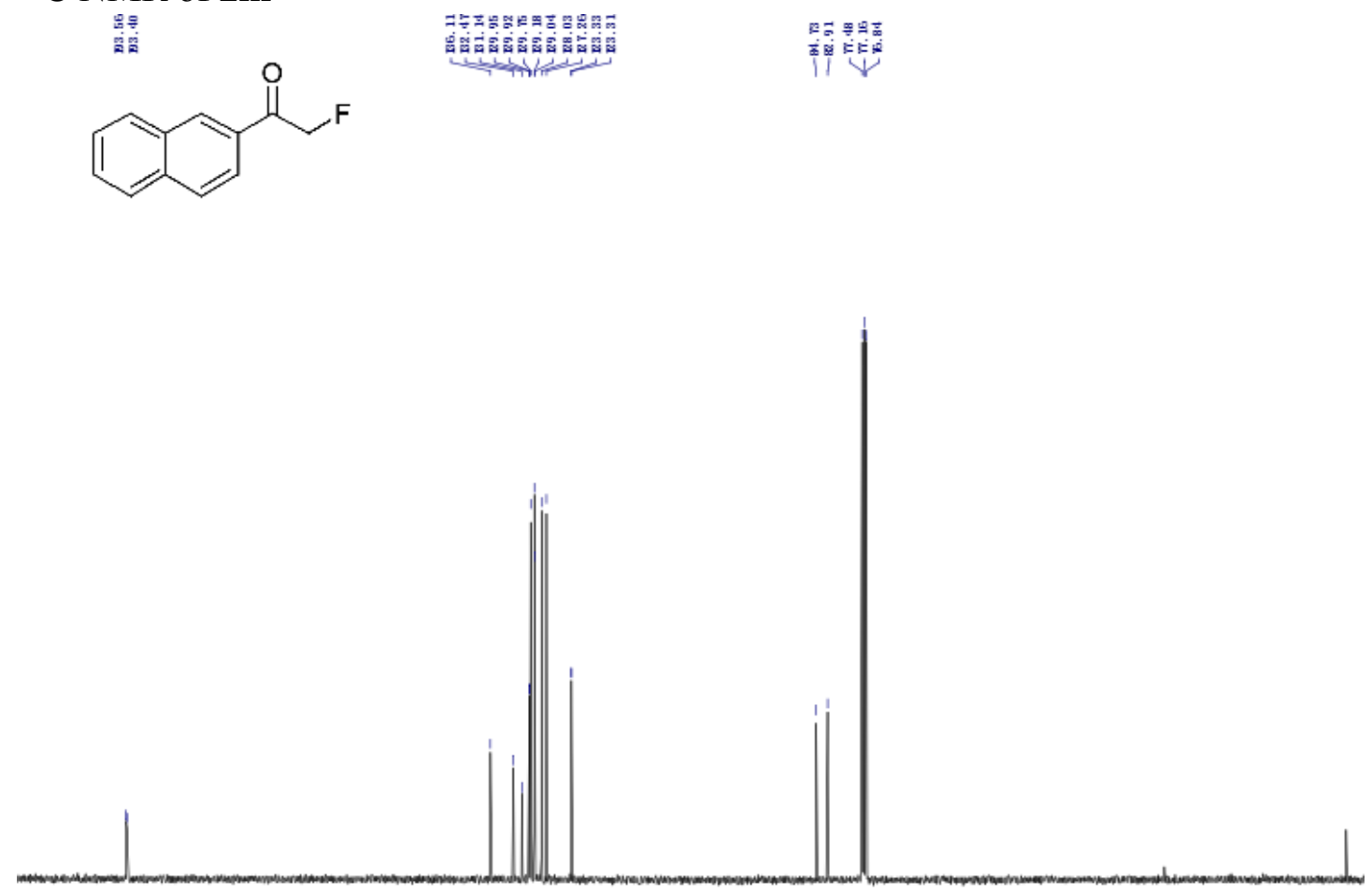

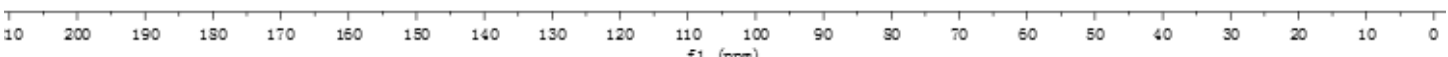


${ }^{19} \mathrm{~F}$ NMR of $\mathbf{2 m}$

g:

สุ่ สี่<smiles>O=C(CF)c1ccc2ccccc2c1</smiles>

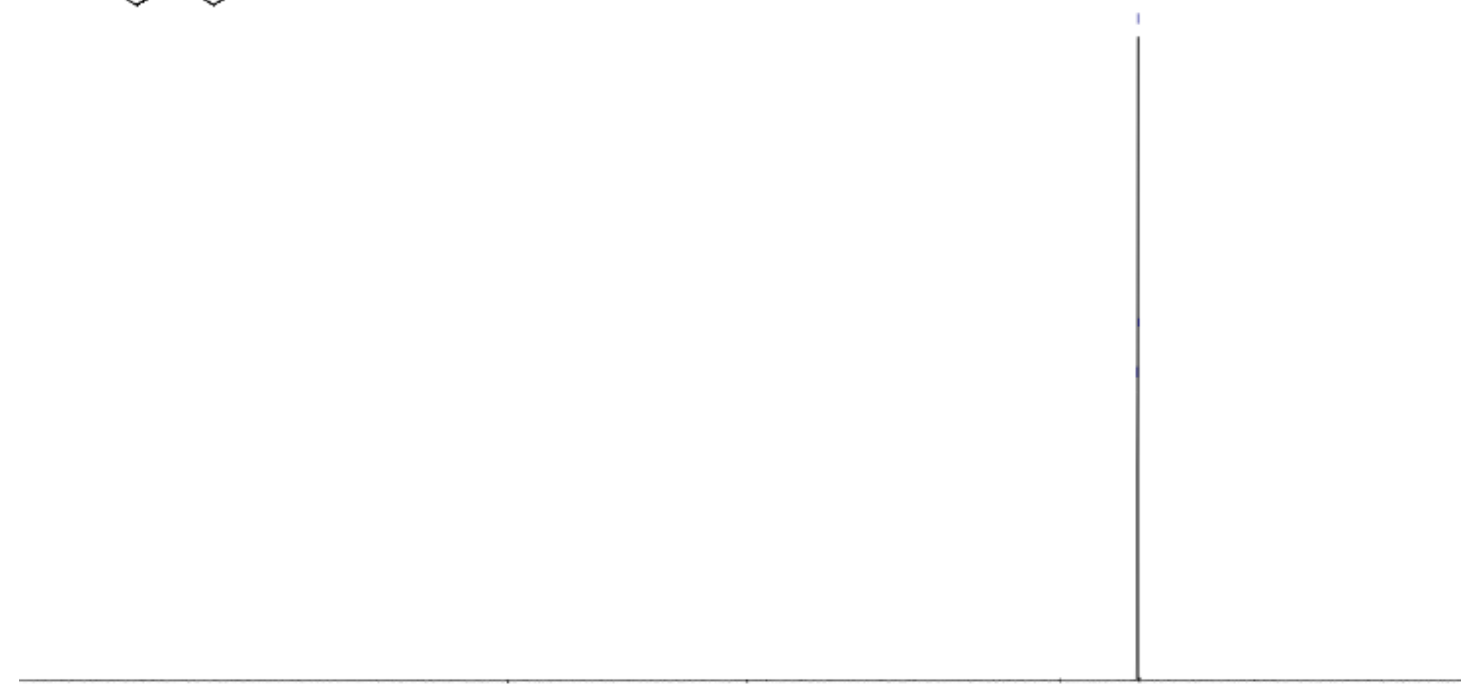

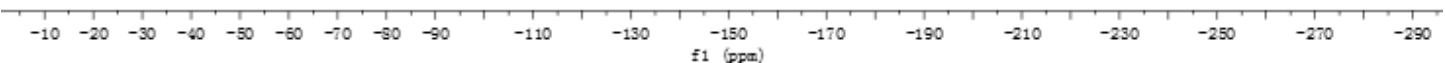

${ }^{1} \mathrm{H}$ NMR of $\mathbf{2 n}$
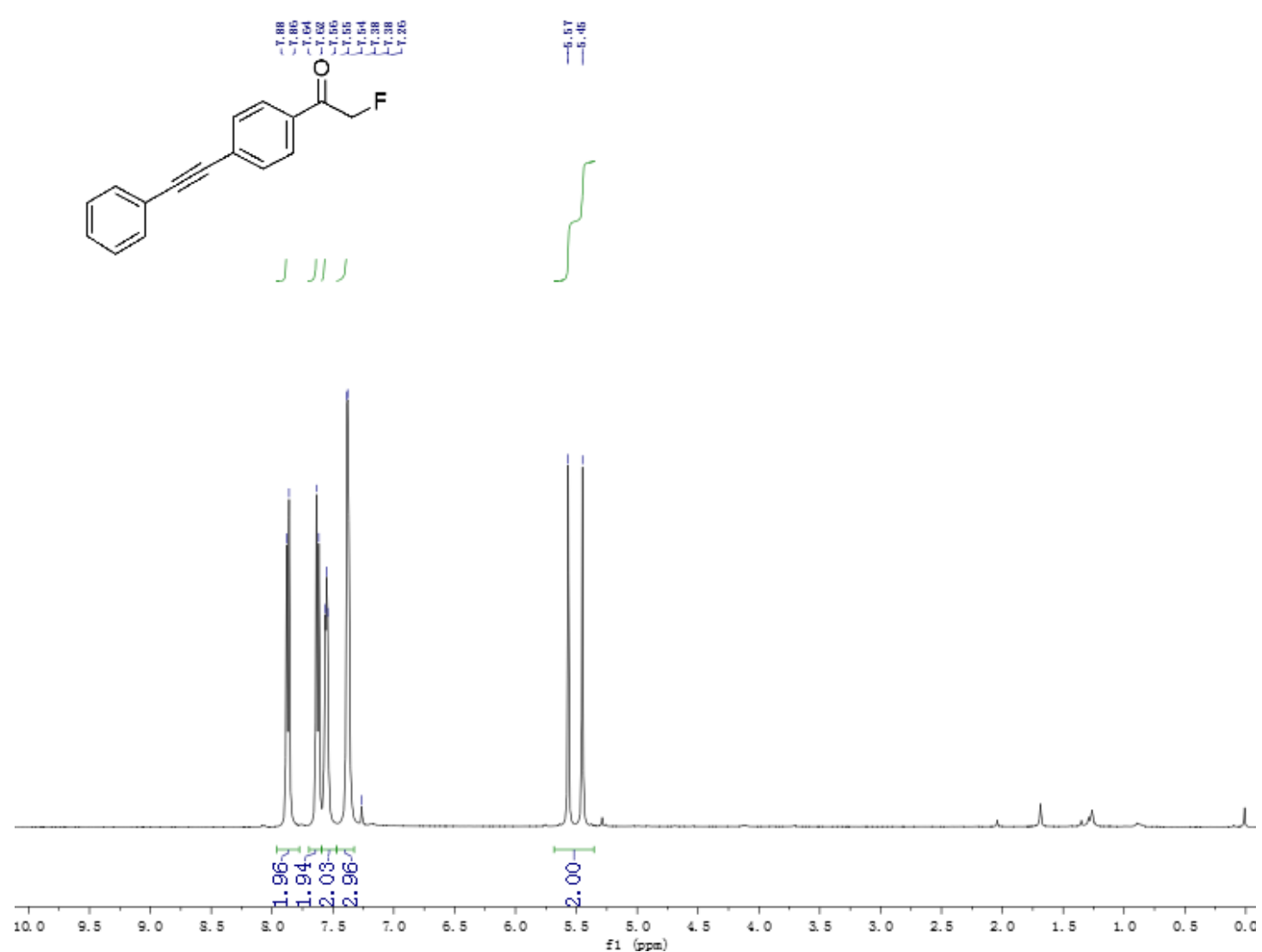
${ }^{13} \mathrm{C}$ NMR of $2 \mathbf{n}$
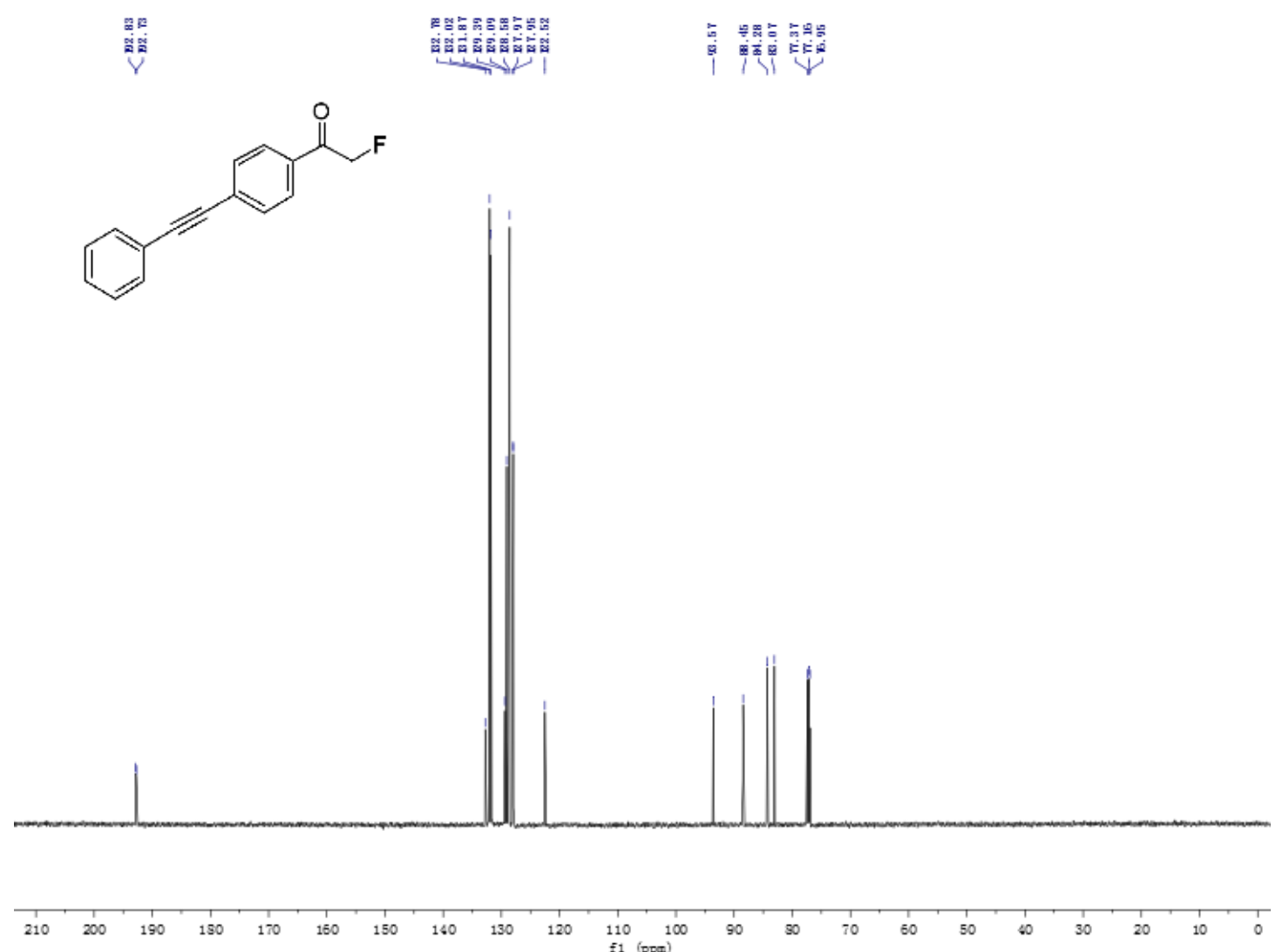

${ }^{19} \mathrm{~F}$ NMR of $\mathbf{2 m}$

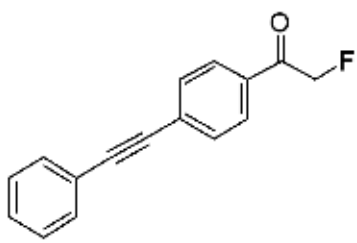

ำ

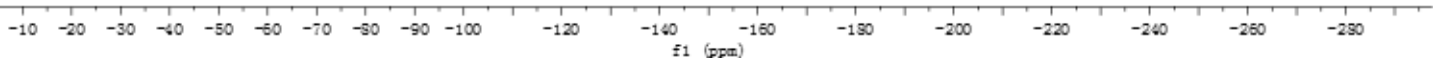


${ }^{1} \mathrm{H}$ NMR of $2 \mathbf{0}$
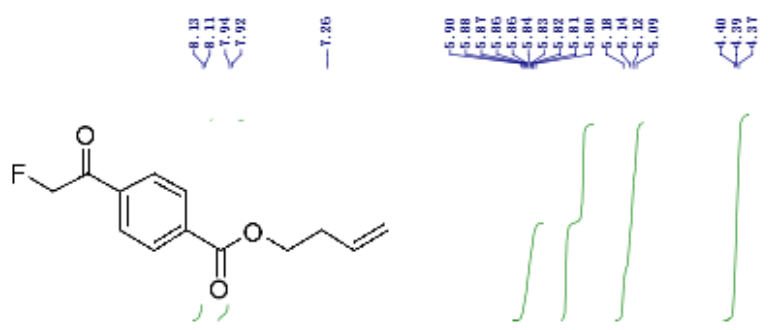

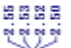

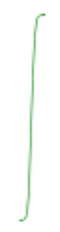

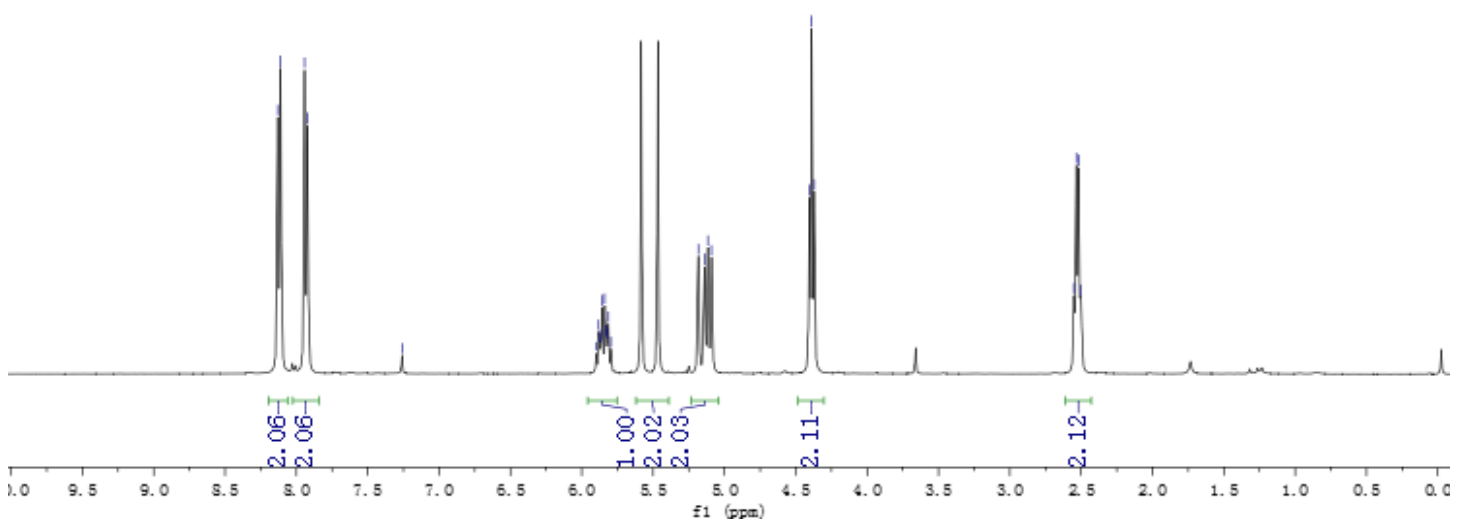

${ }^{13} \mathrm{C}$ NMR of 20

啇的

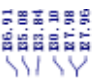

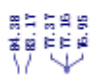

$\stackrel{9}{1}$
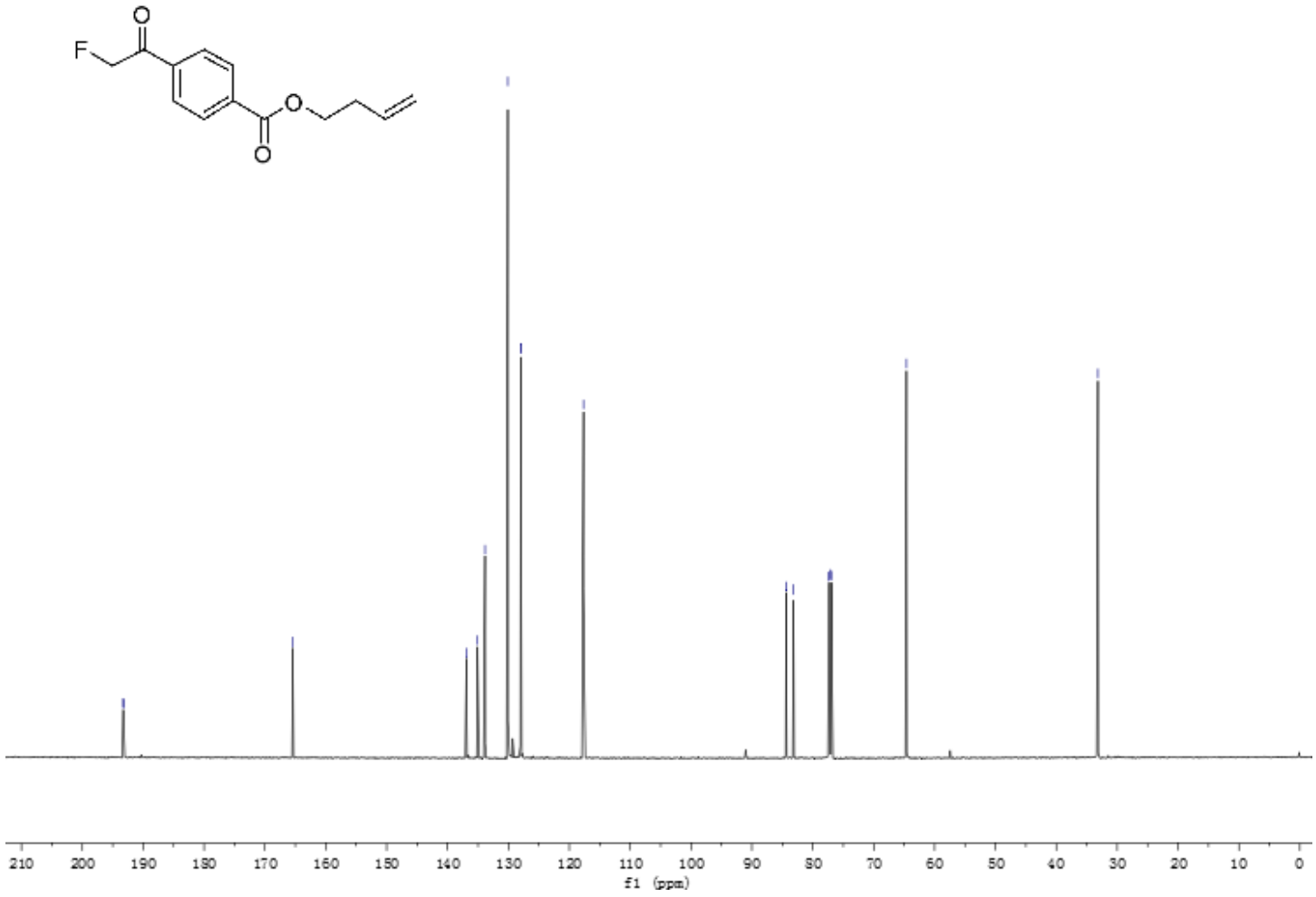

66 
${ }^{19} \mathrm{~F}$ NMR of $2 \mathbf{0}$

ํㅜㄴ
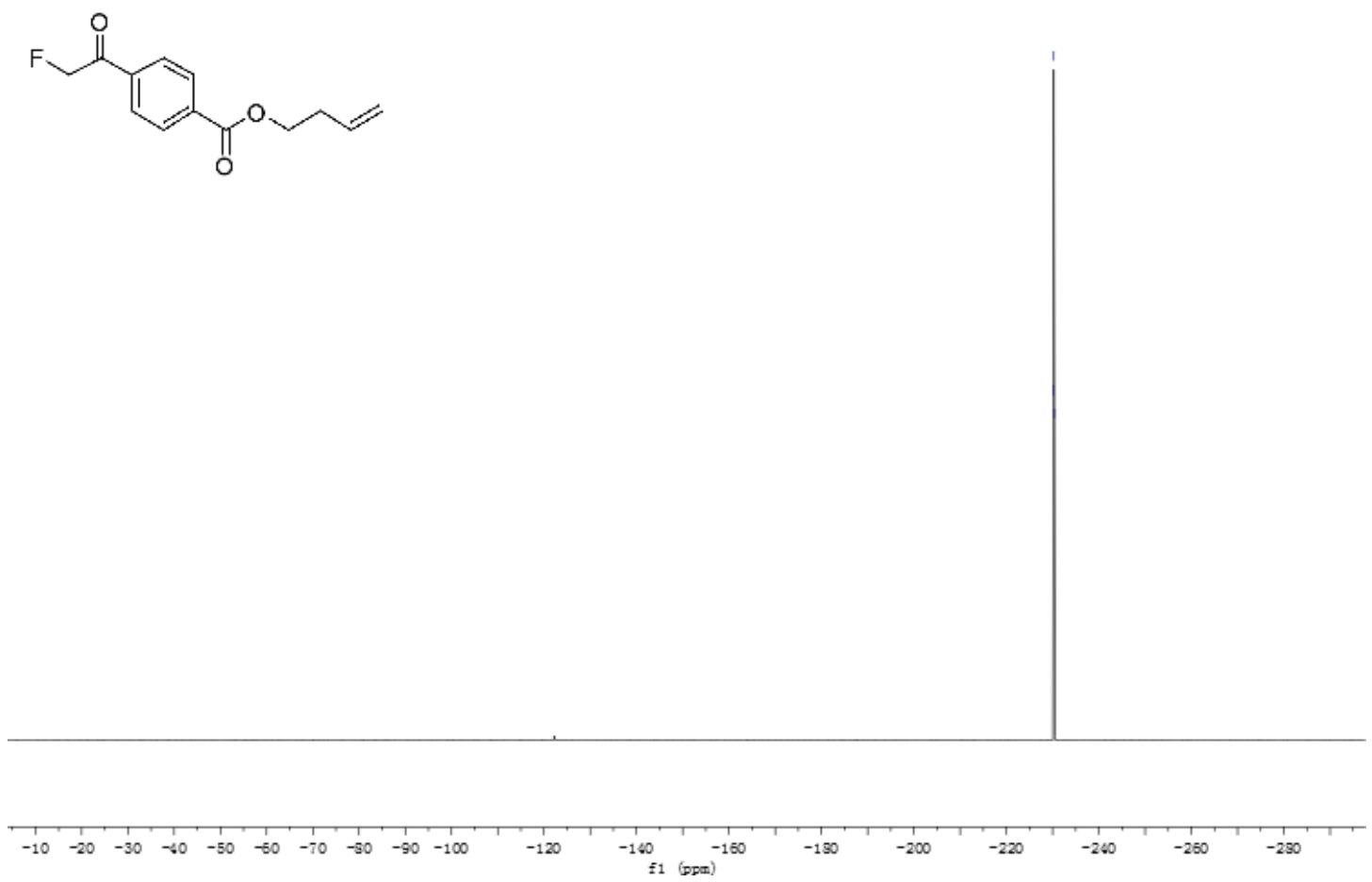

${ }^{1} \mathrm{H}$ NMR of $\mathbf{2 p}$

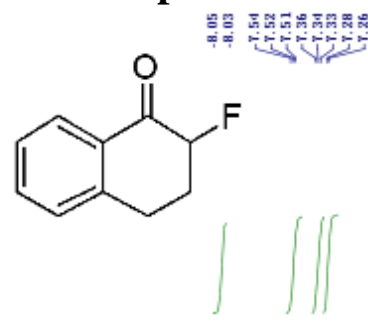

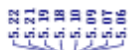

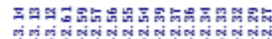
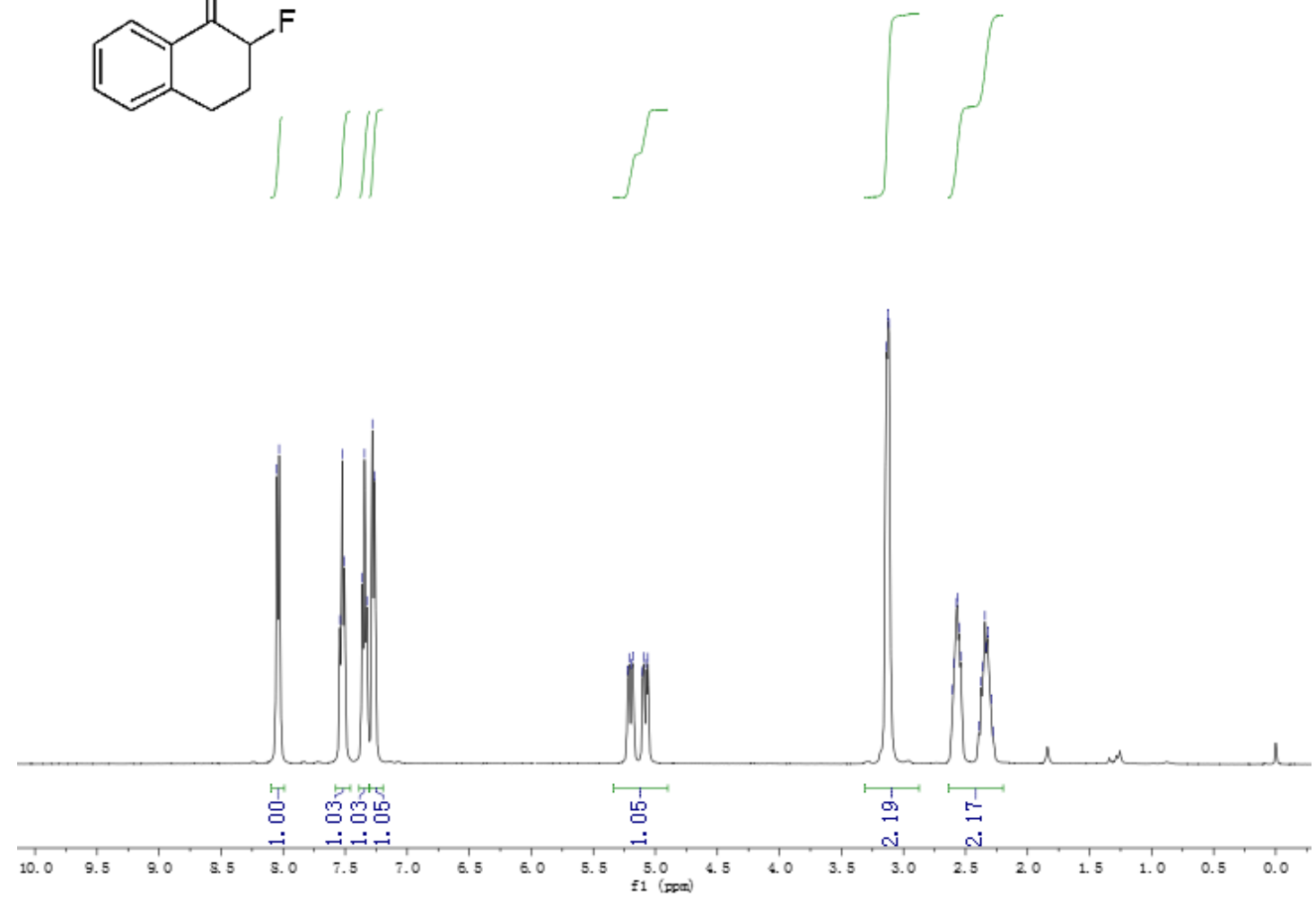

67 
${ }^{13} \mathrm{C}$ NMR of $\mathbf{2 p}$

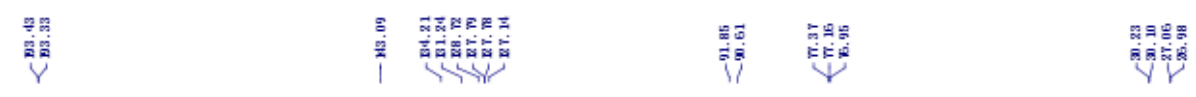<smiles>O=C1c2ccccc2CCC1F</smiles>
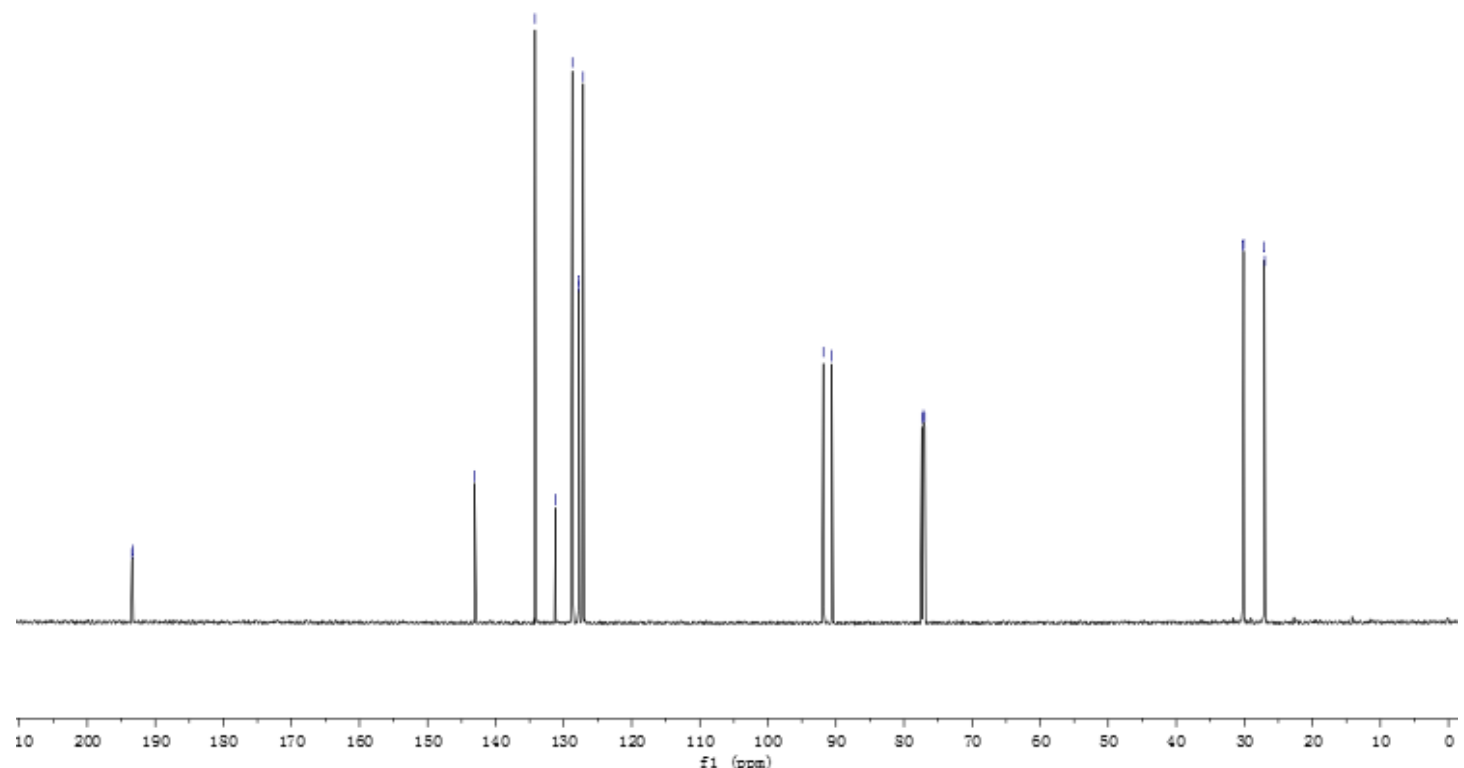

${ }^{19}$ F NMR of 2p

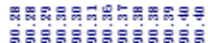

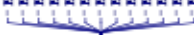<smiles>O=C1c2ccccc2CCC1F</smiles> 
${ }^{1} \mathrm{H}$ NMR of $\mathbf{2 q}$

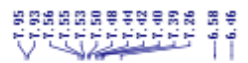<smiles>O=C(c1ccccc1)C(F)c1ccccc1</smiles>

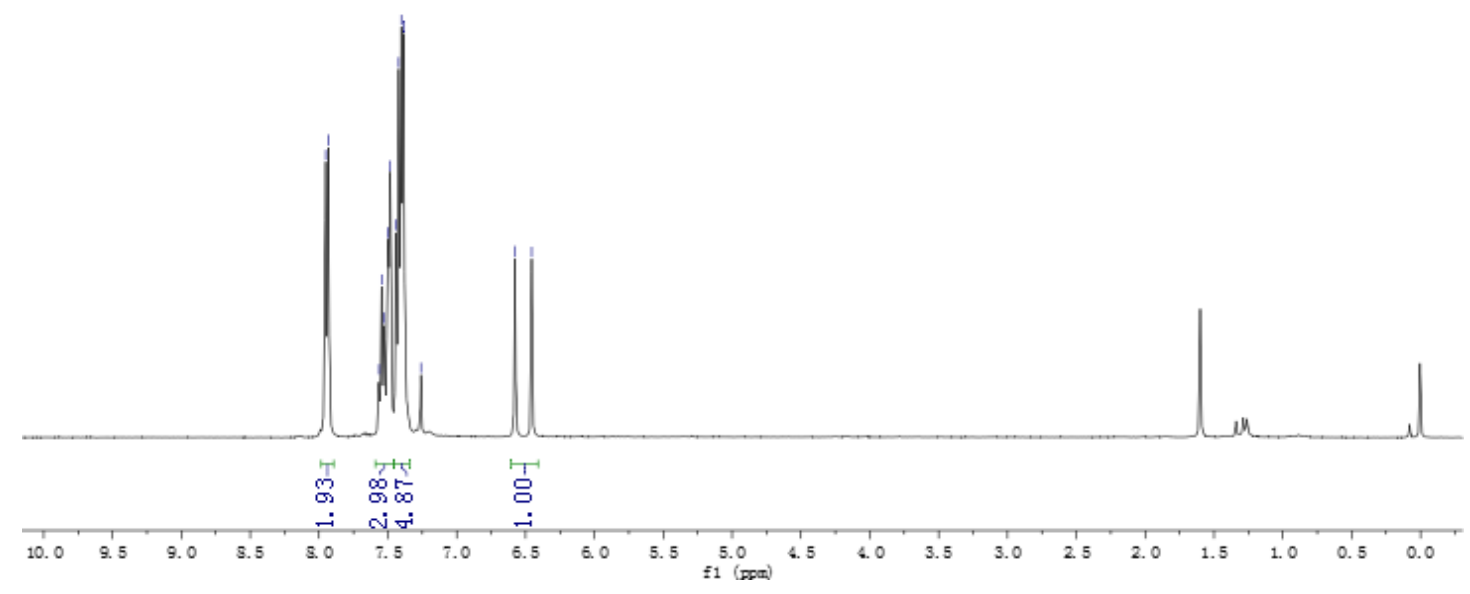

${ }^{13} \mathrm{C}$ NMR of $\mathbf{2 q}$

商

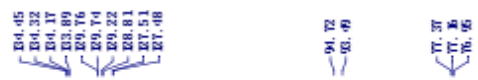<smiles>O=C(c1ccccc1)C(F)c1ccccc1</smiles>

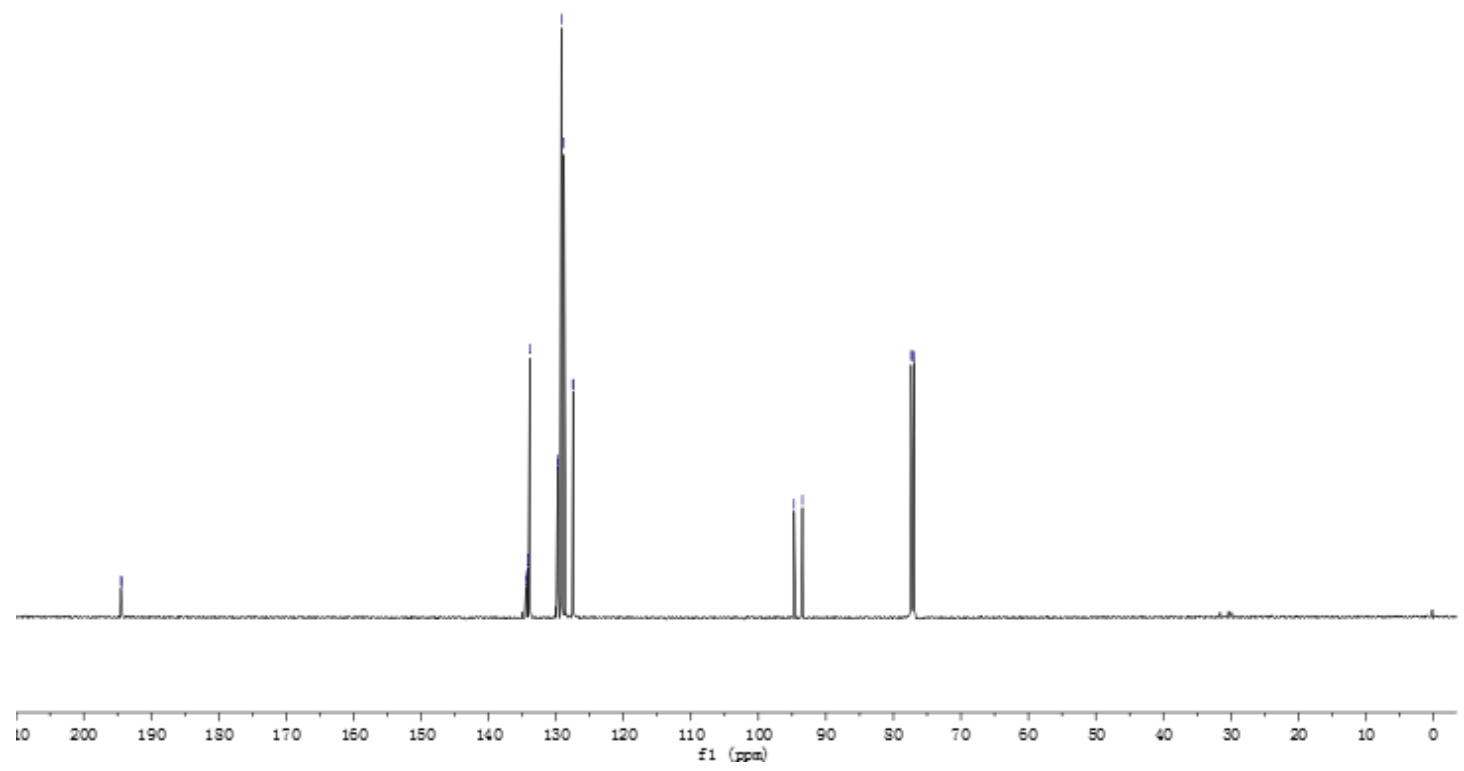


${ }^{19}$ F NMR of 2q<smiles>O=C(c1ccccc1)C(F)c1ccccc1</smiles>

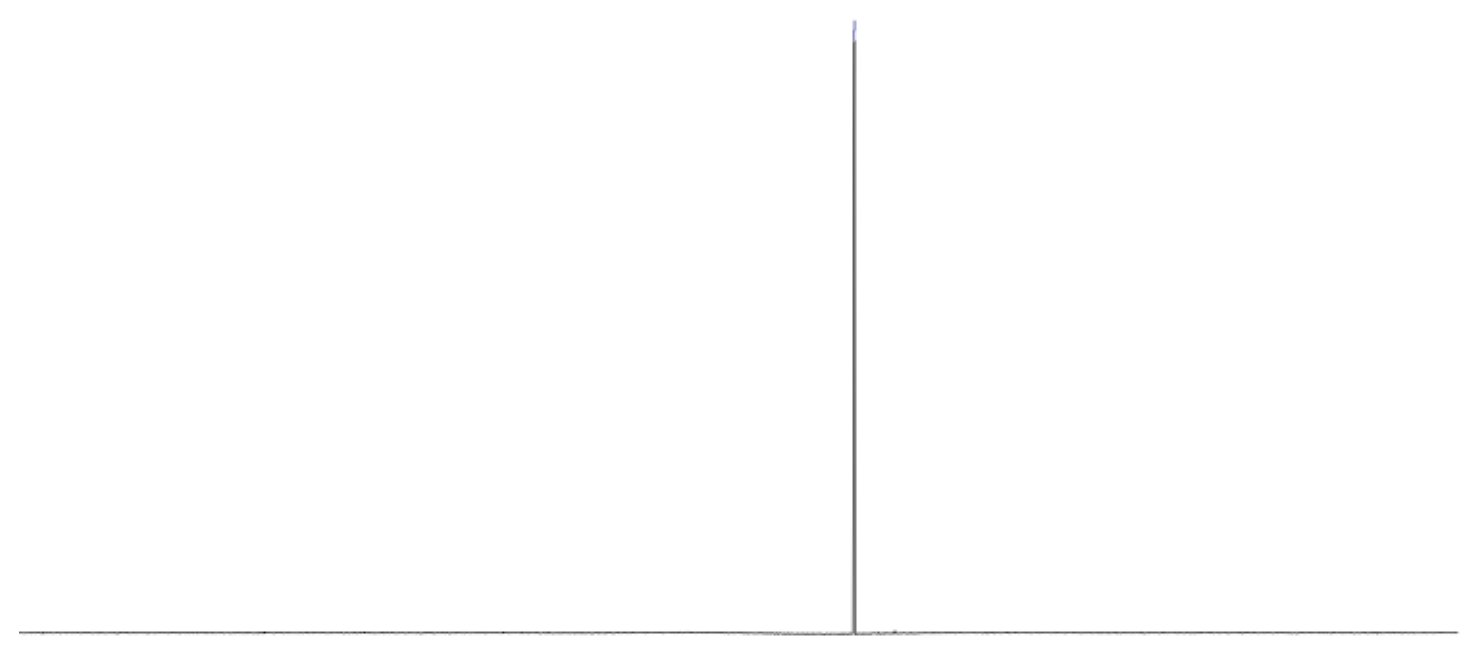

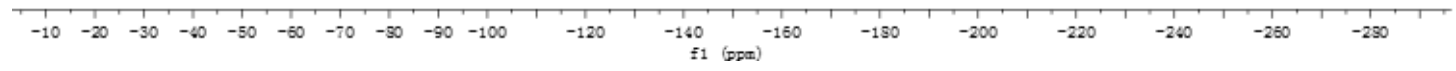

${ }^{1} \mathrm{H}$ NMR of $\mathbf{2 r}$

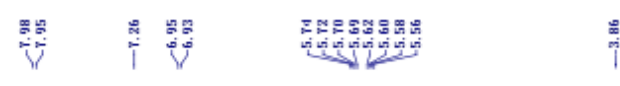<smiles>COc1ccc(C(=O)C(C)F)cc1</smiles>

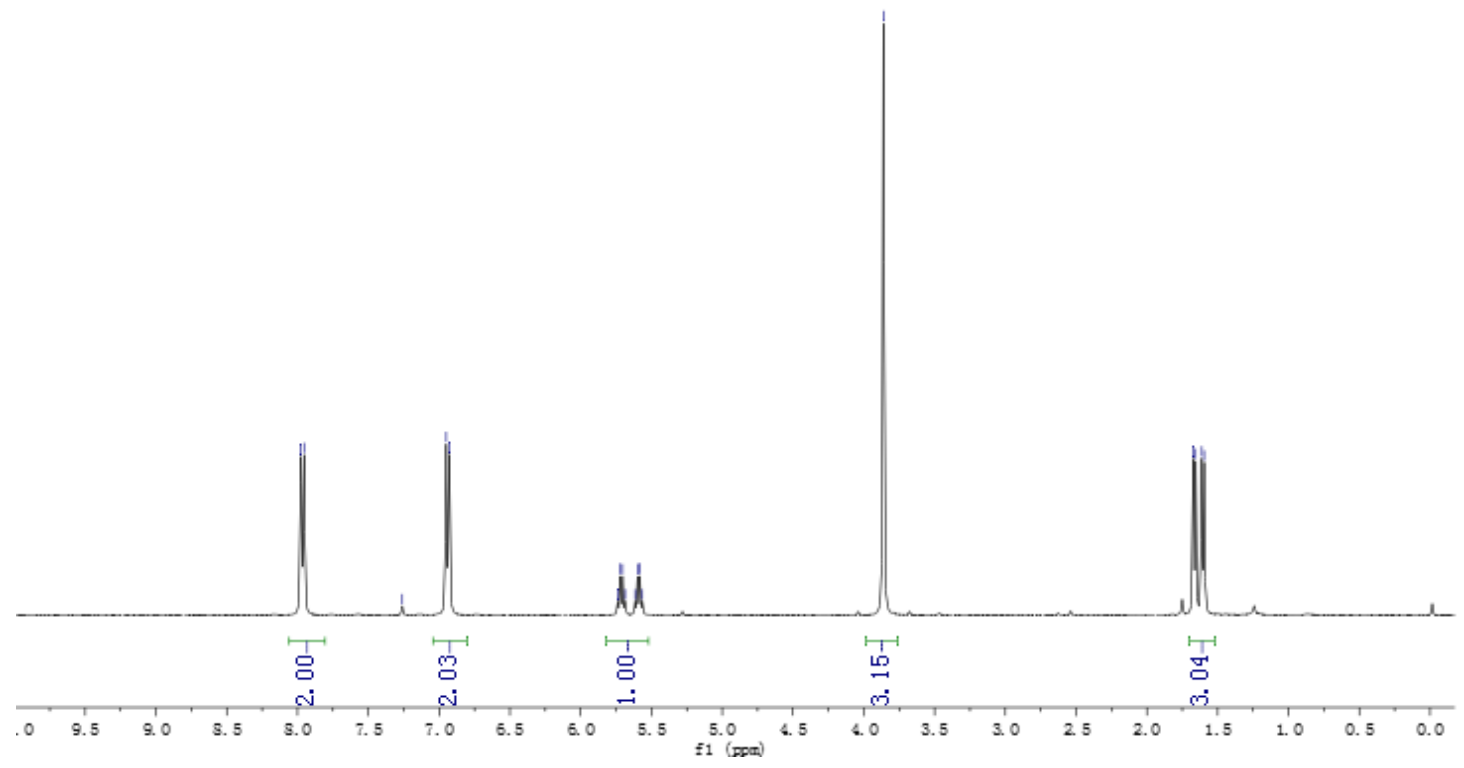




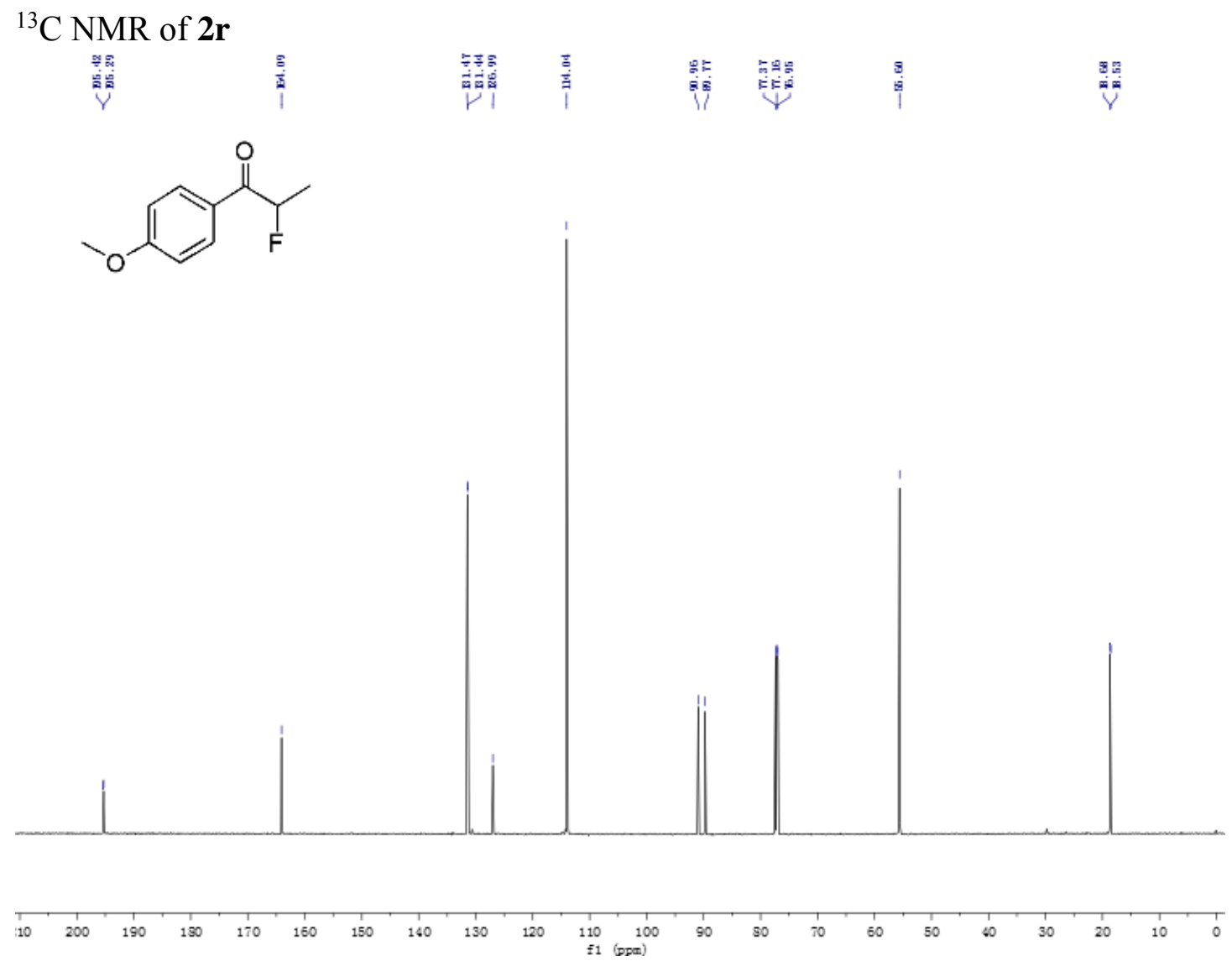

${ }^{19} \mathrm{~F}$ NMR of $2 \mathbf{r}$

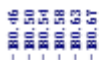<smiles>COc1ccc(C(=O)C(C)F)cc1</smiles>

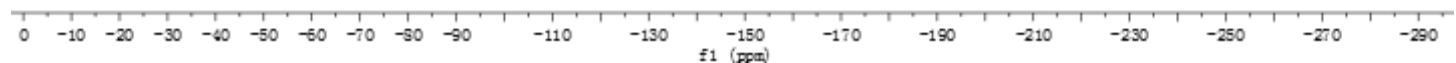


${ }^{1} \mathrm{H}$ NMR of $2 \mathrm{~s}$
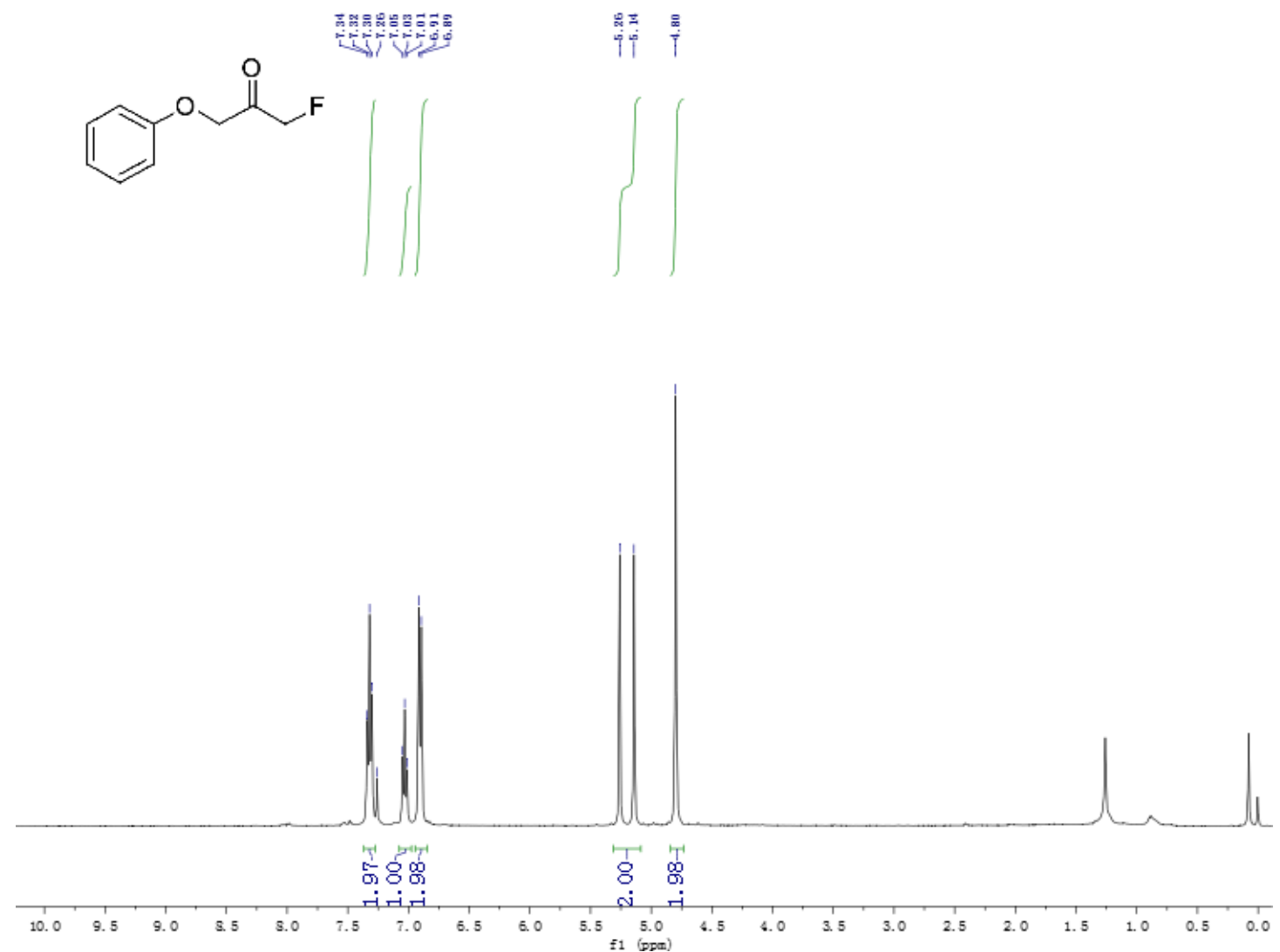

${ }^{13} \mathrm{C}$ NMR of $2 \mathrm{~s}$

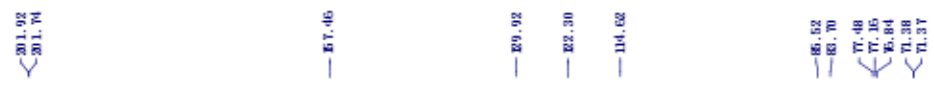<smiles>O=C(CF)COc1ccccc1</smiles>

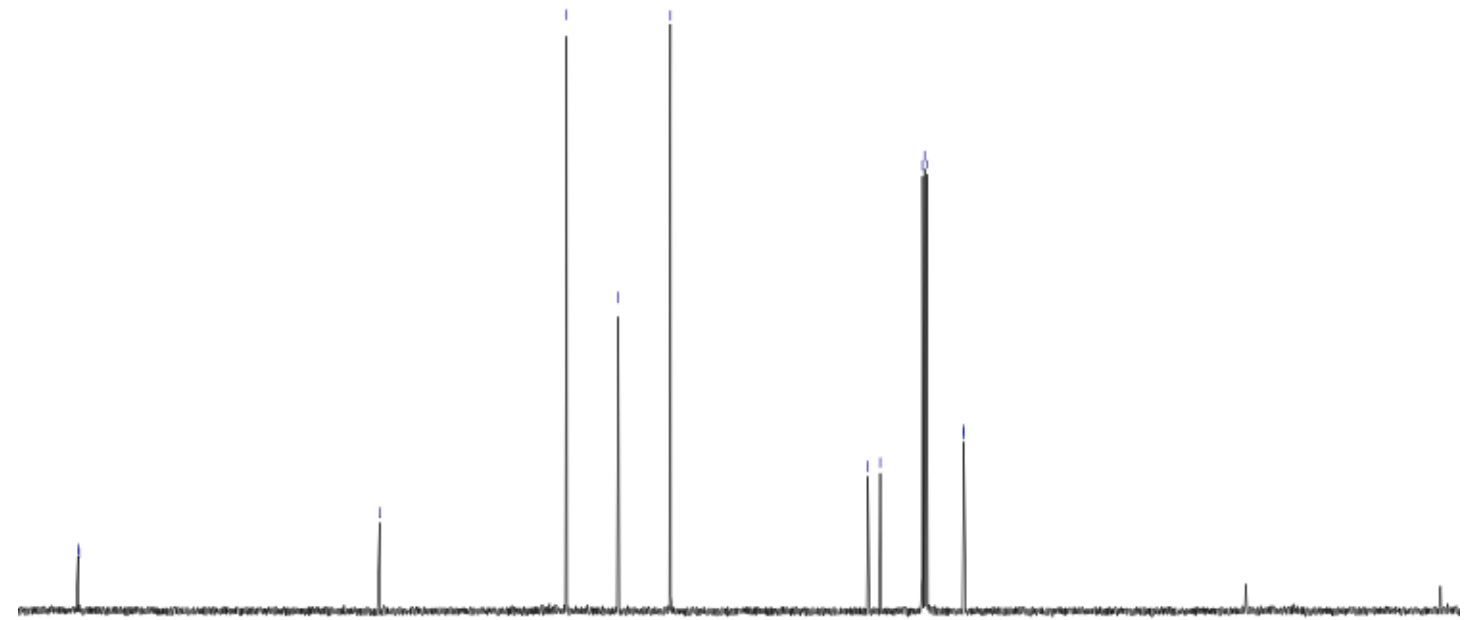

\begin{tabular}{lllllllllllllllllllllllllllllllllll}
\hline 10 & 200 & 190 & 130 & 170 & 160 & 150 & 140 & 130 & 120 & 110 & 100 & 90 & 50 & 70 & 60 & 50 & 40 & 30 & 20 & 10 & 0
\end{tabular} 
${ }^{19} \mathrm{~F}$ NMR of $2 \mathrm{~s}$

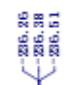<smiles>O=C(CF)COc1ccccc1</smiles>

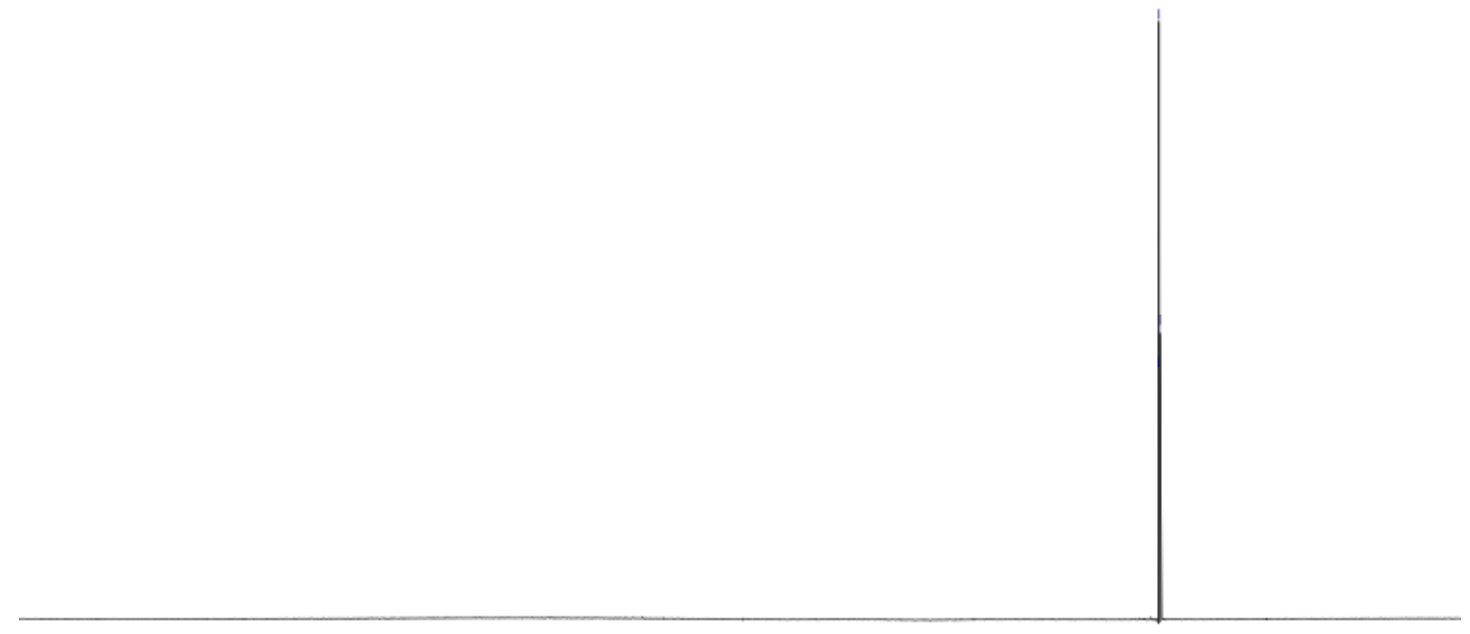

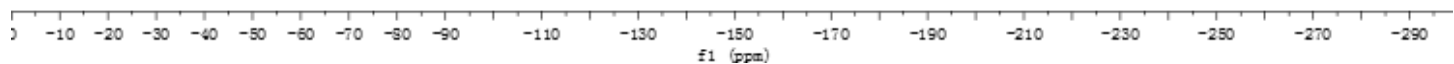

${ }^{1} \mathrm{H}$ NMR of $\mathbf{2 t}$

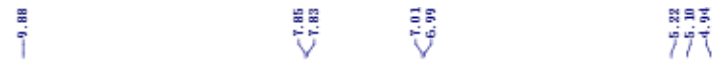
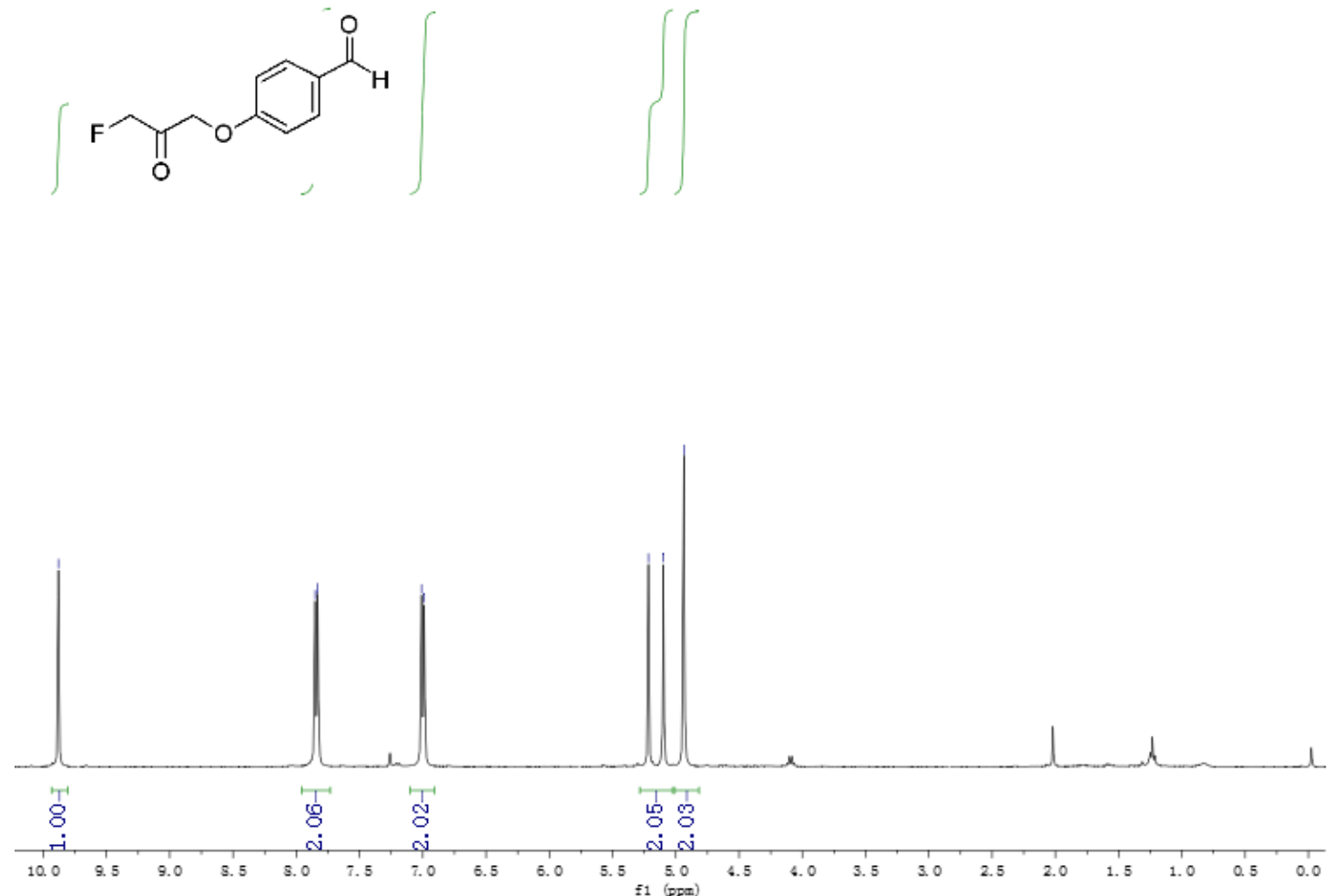
${ }^{13} \mathrm{C}$ NMR of $2 t$

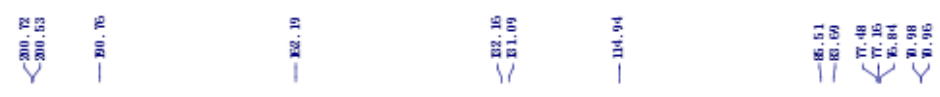

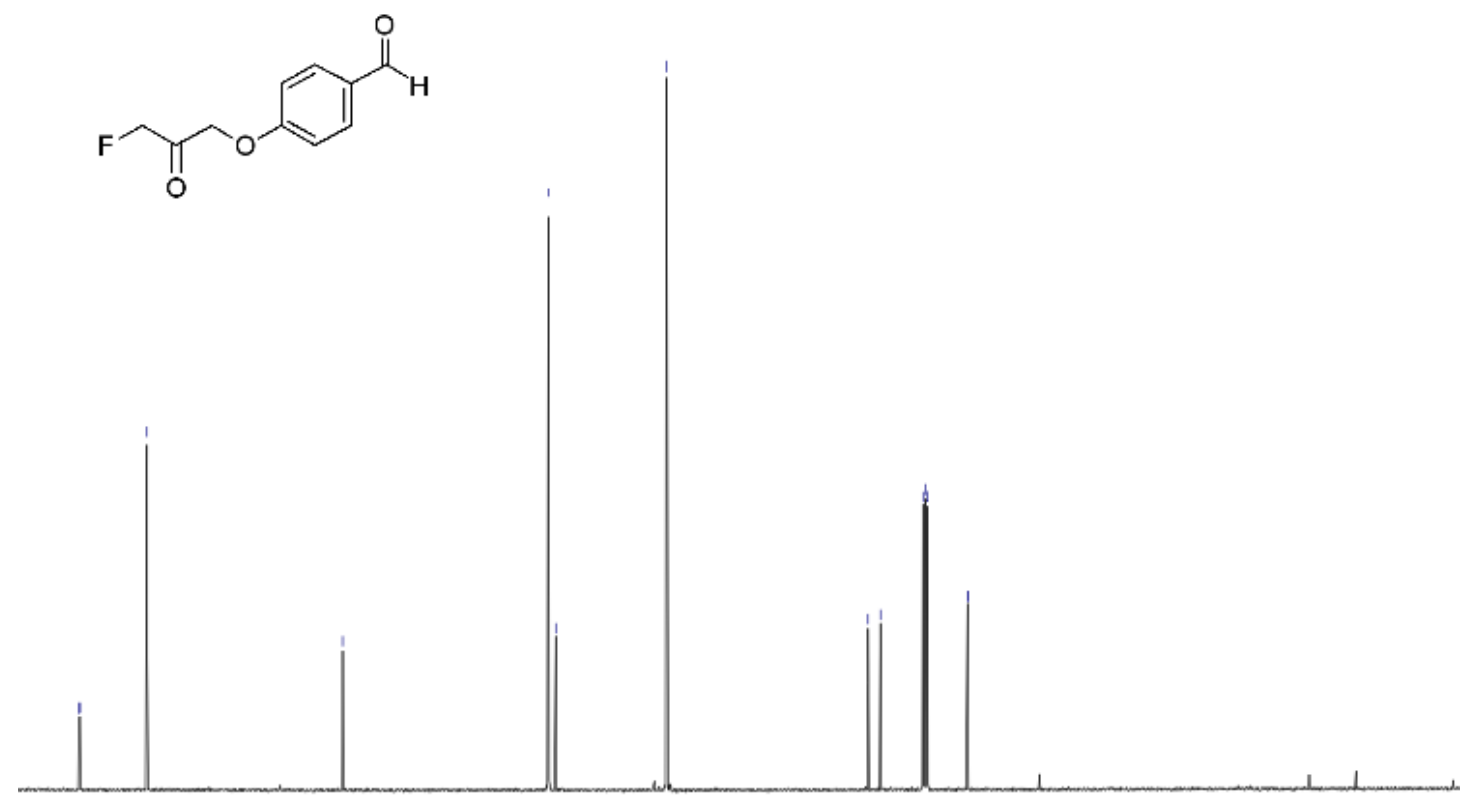

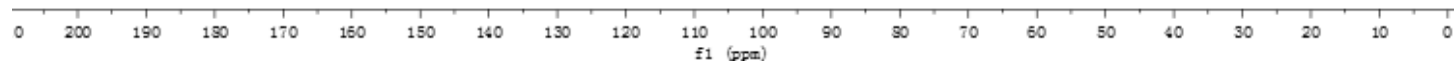

${ }^{19} \mathrm{~F}$ NMR of $\mathbf{2 t}$

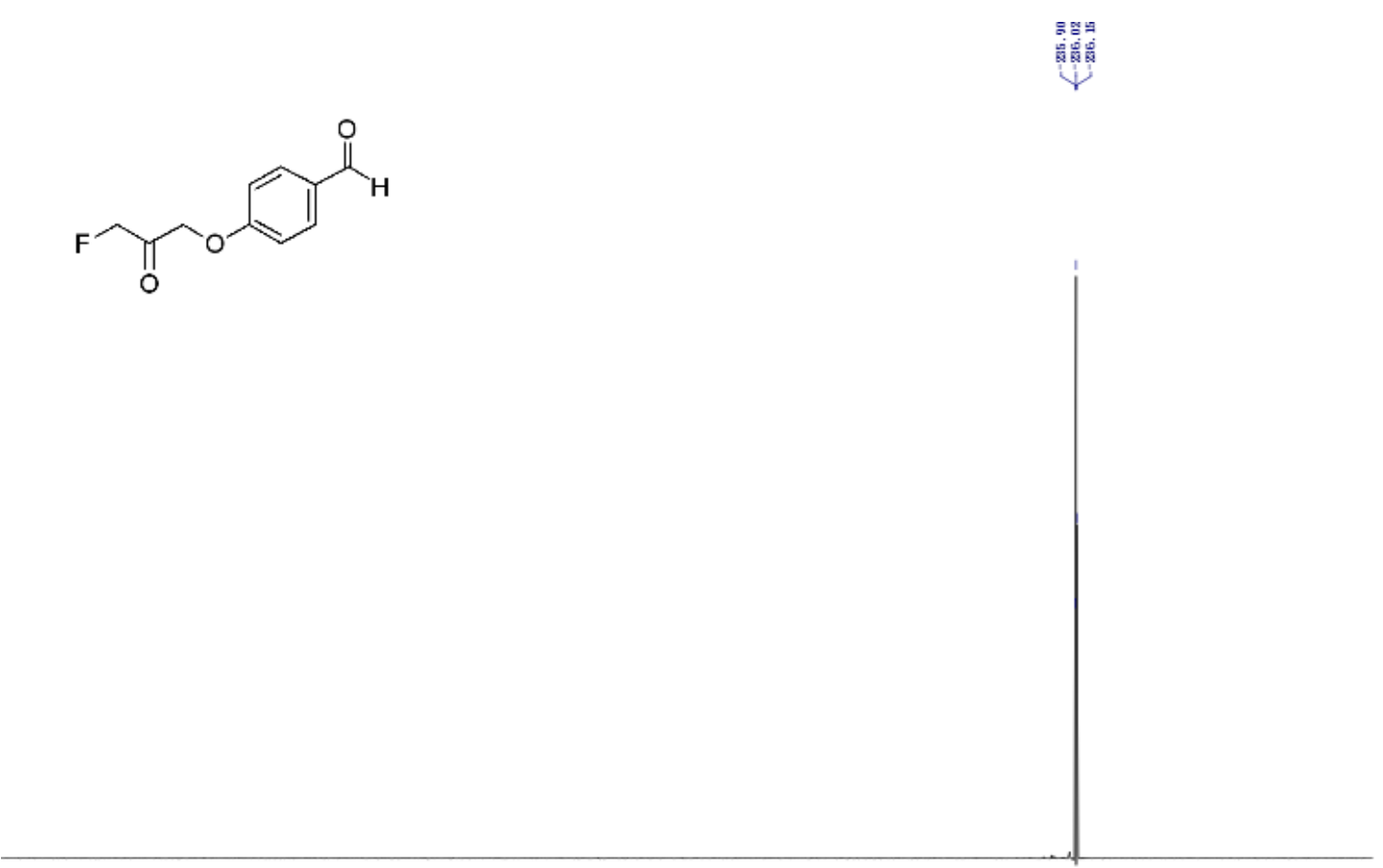

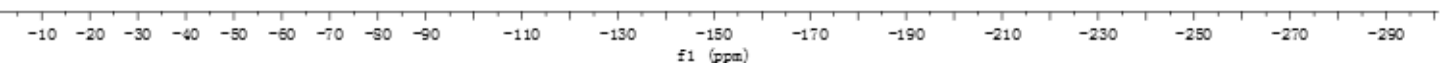


${ }^{1} \mathrm{H}$ NMR of $\mathbf{2 u}$

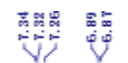

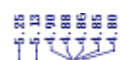

$\stackrel{8}{i}$<smiles>CC(O)c1ccc(OCC(=O)CF)cc1</smiles>

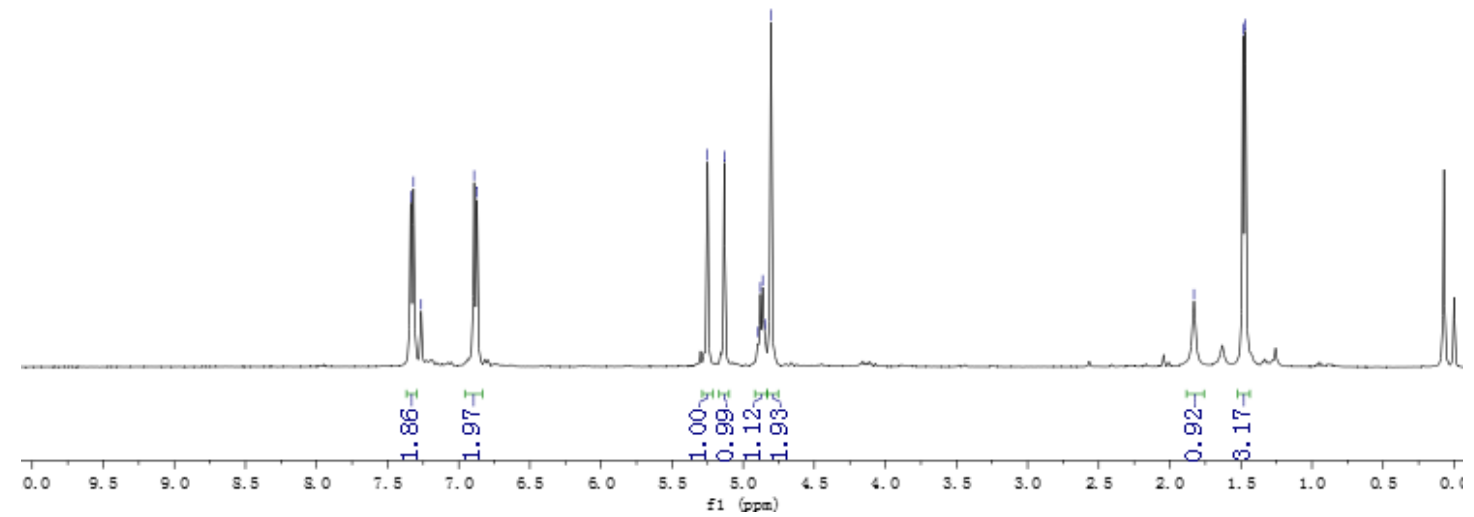

${ }^{13} \mathrm{C}$ NMR of $\mathbf{2 u}$
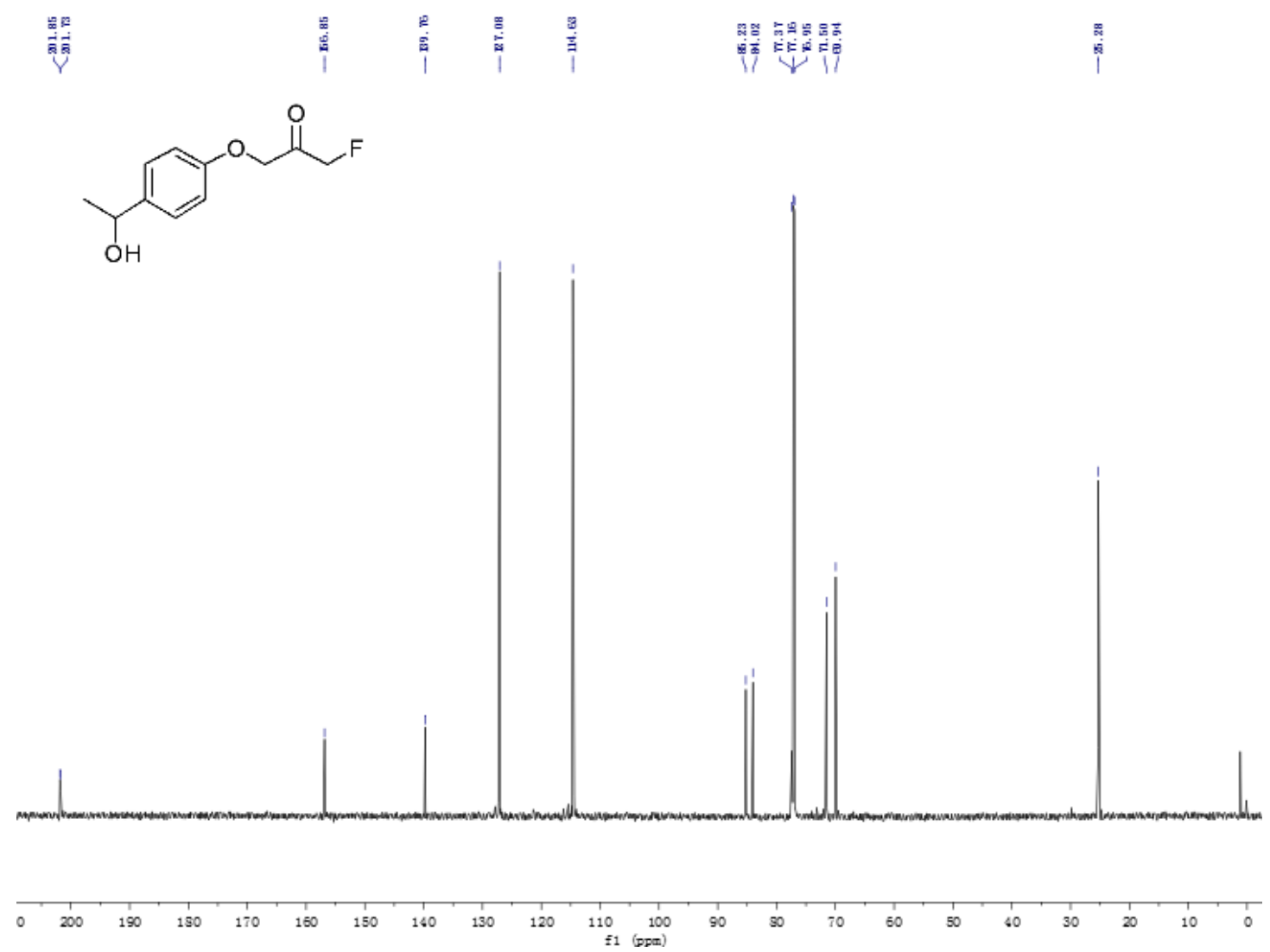
${ }^{19} \mathrm{~F}$ NMR of $\mathbf{2 u}$

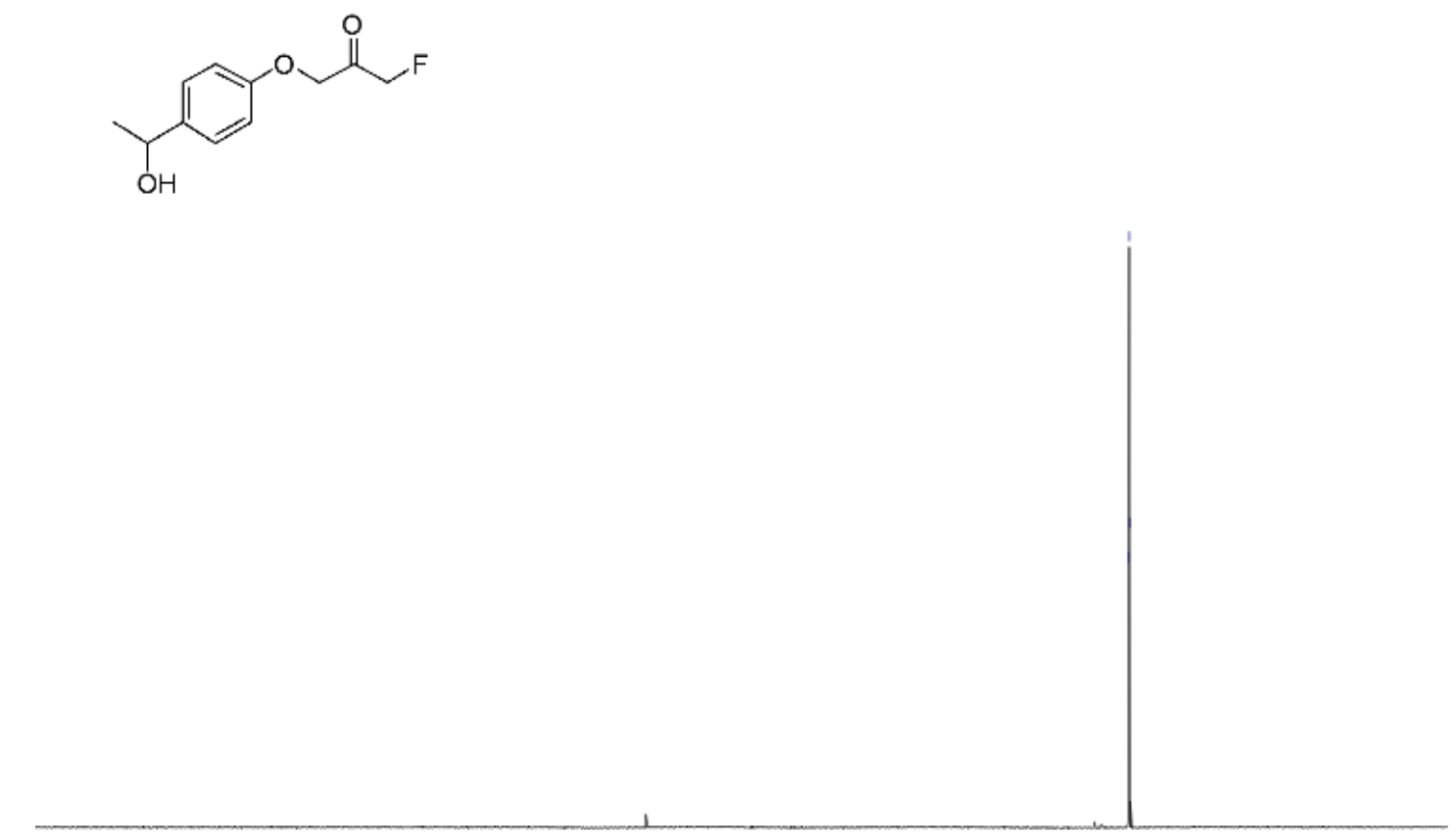

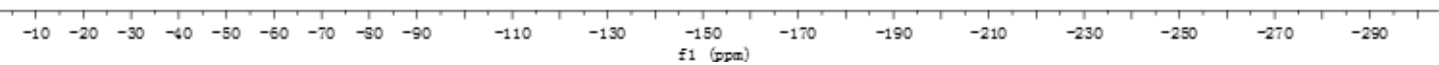

${ }^{1} \mathrm{H}$ NMR of $\mathbf{2 v}$

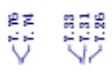<smiles>O=C(CF)CN[As]</smiles>

苫

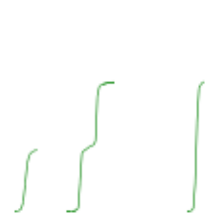

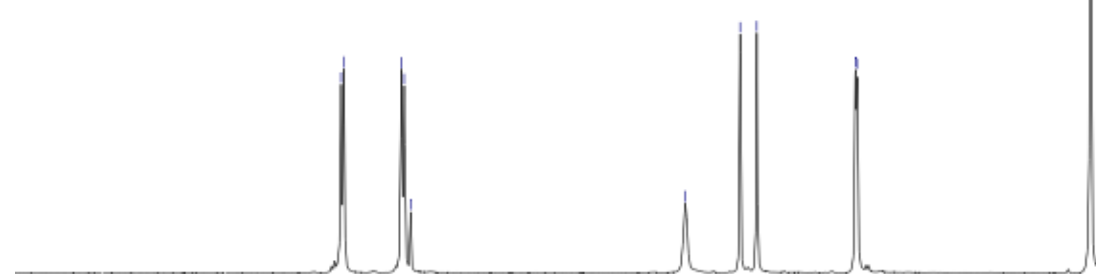

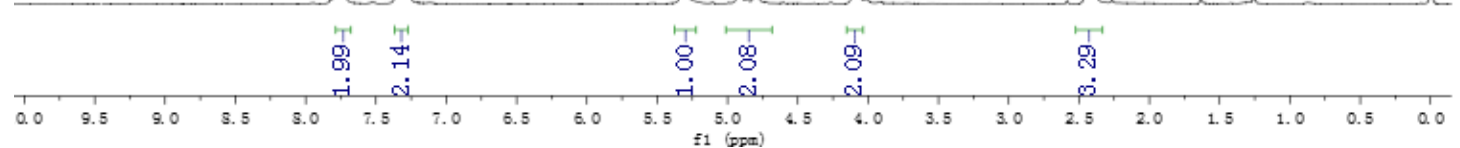




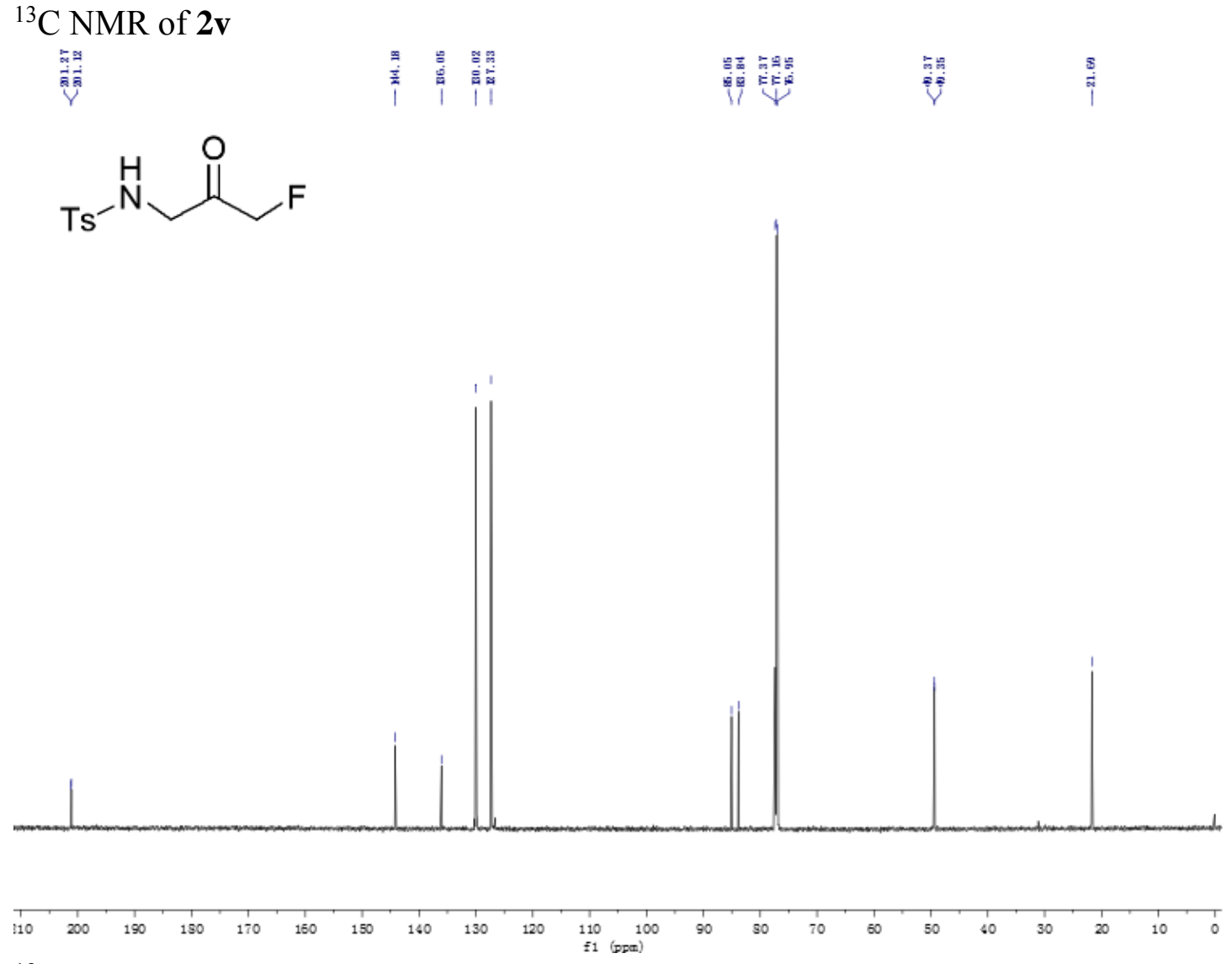

${ }^{19} \mathrm{~F}$ NMR of $\mathbf{2 v}$

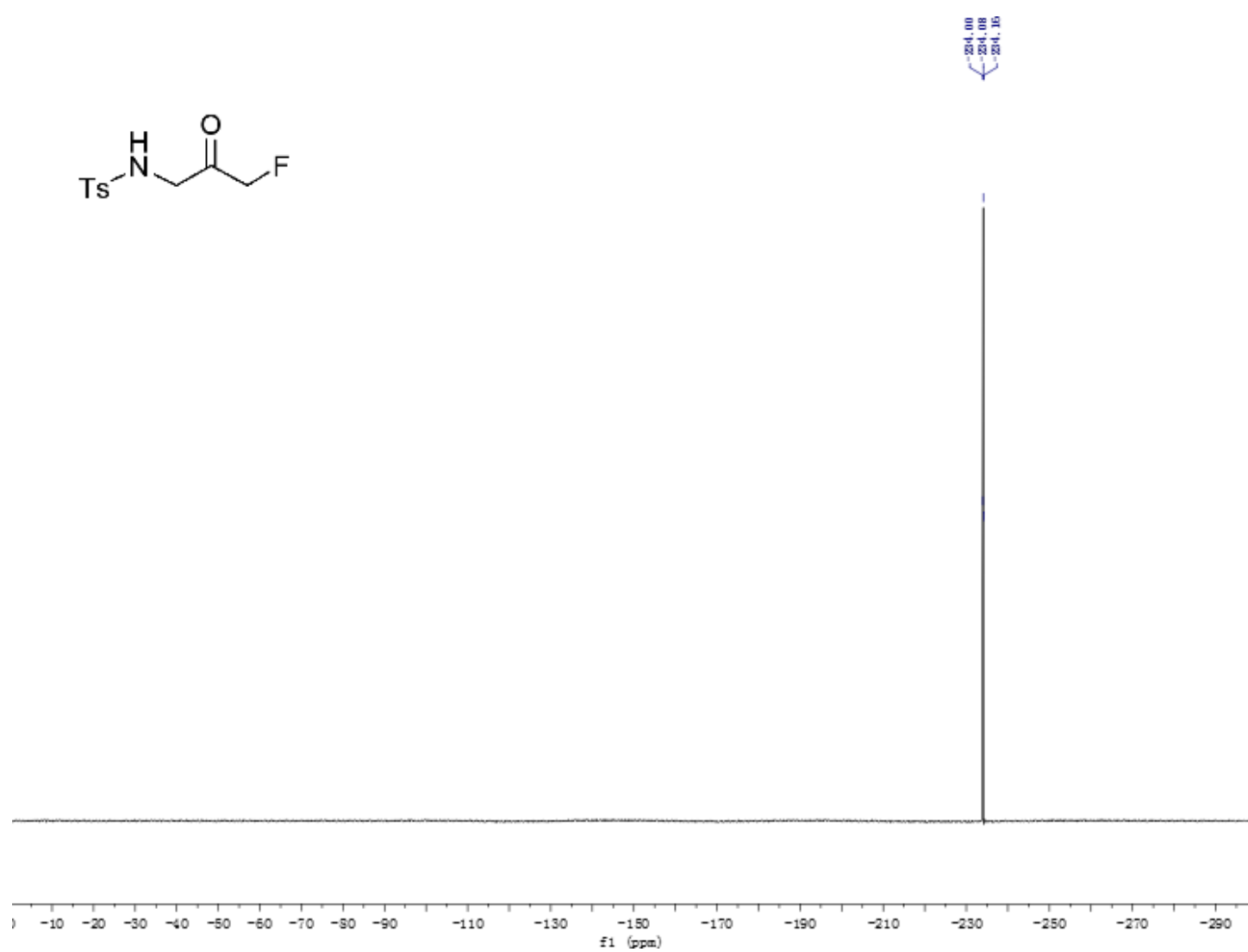


${ }^{1} \mathrm{H}$ NMR of $\mathbf{2 w}$
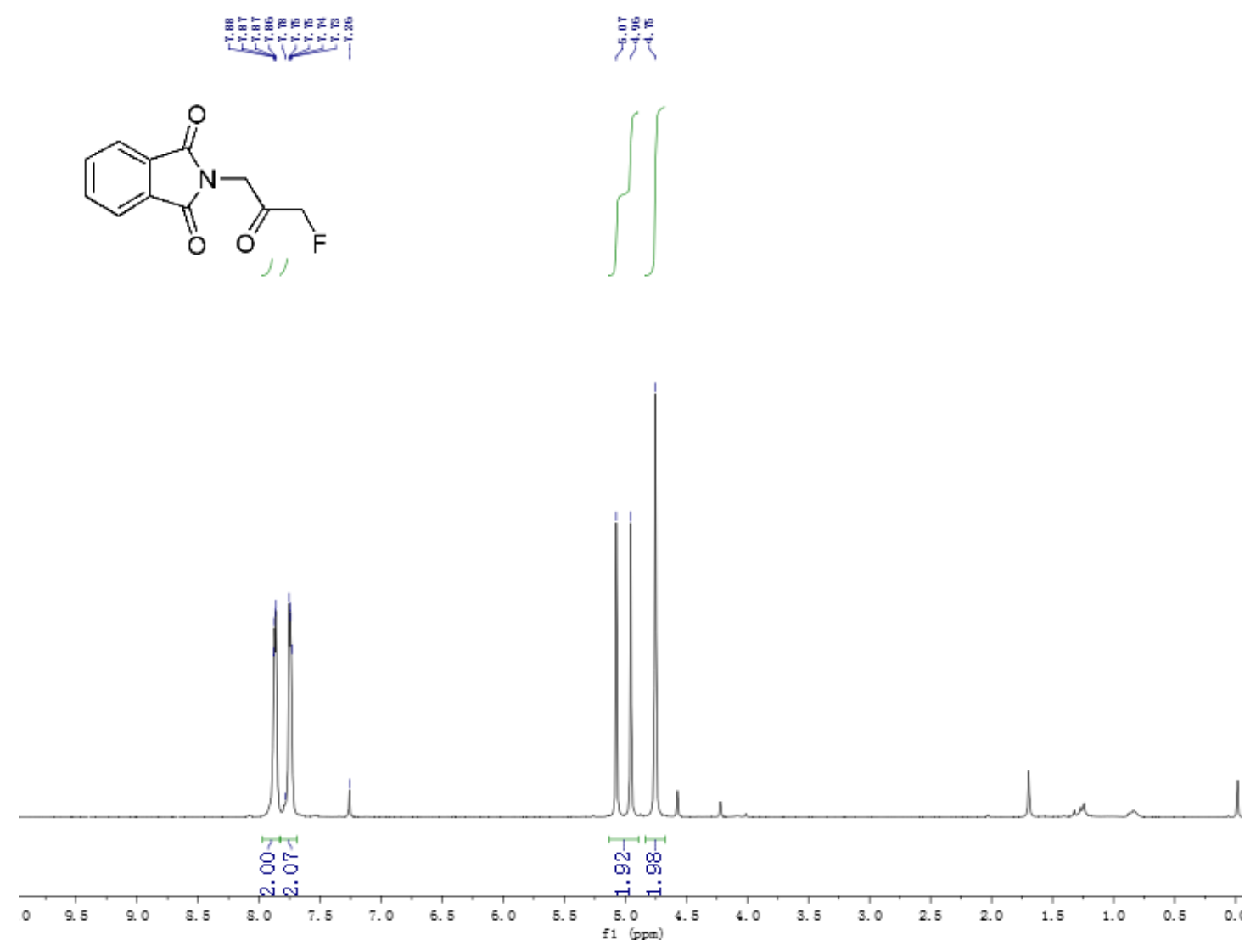

${ }^{13} \mathrm{C}$ NMR of $2 \mathrm{w}$

高跬

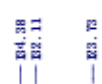

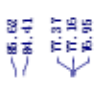

籍
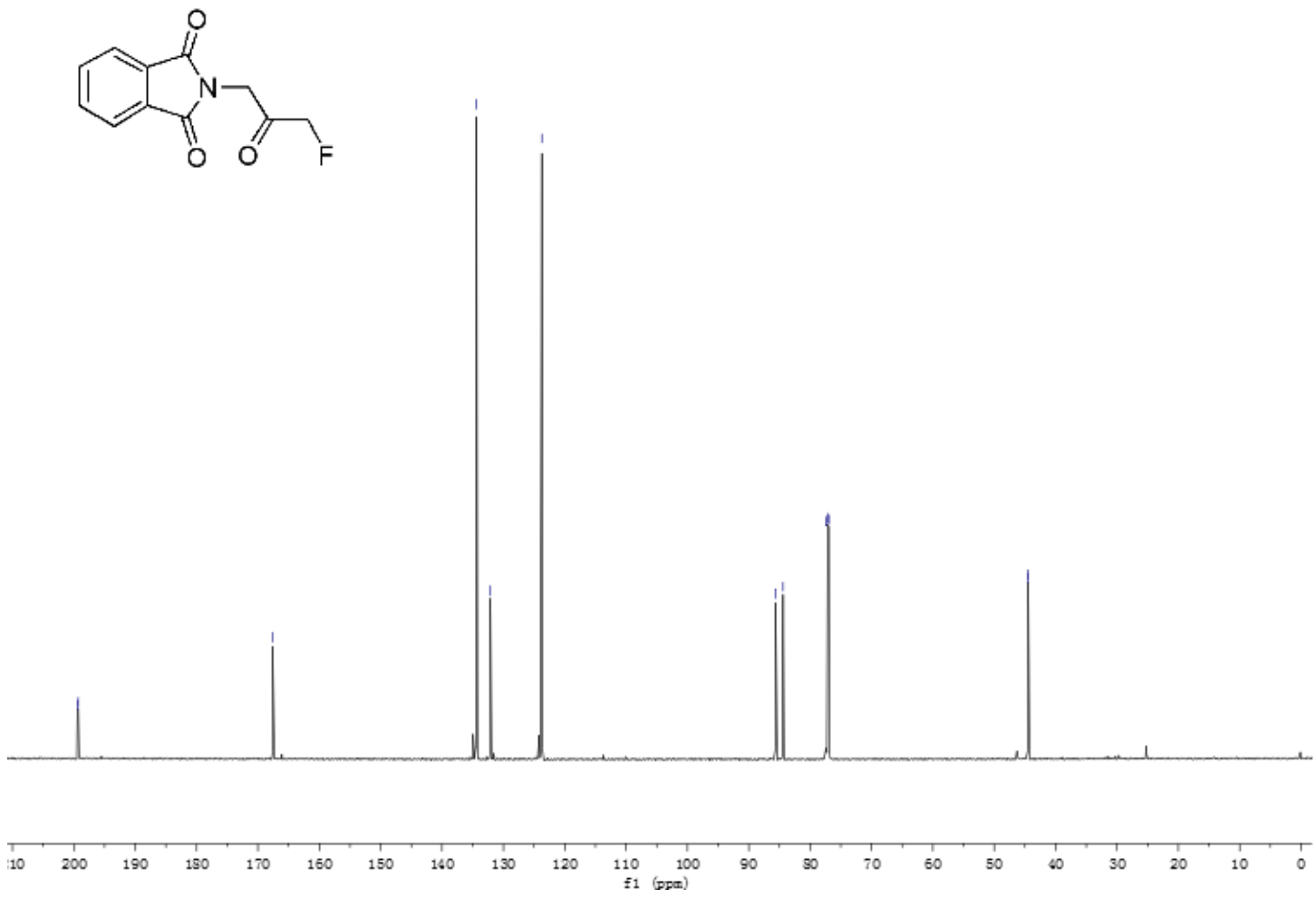

78 
${ }^{19}$ F NMR of $\mathbf{2 w}$

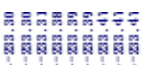

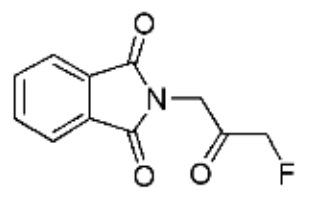

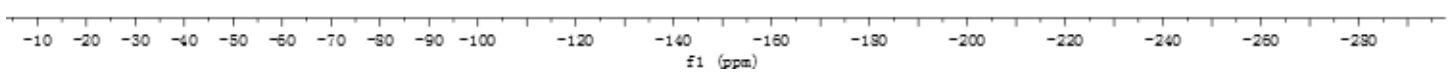

${ }^{1} \mathrm{H}$ NMR of $\mathbf{2 x}$
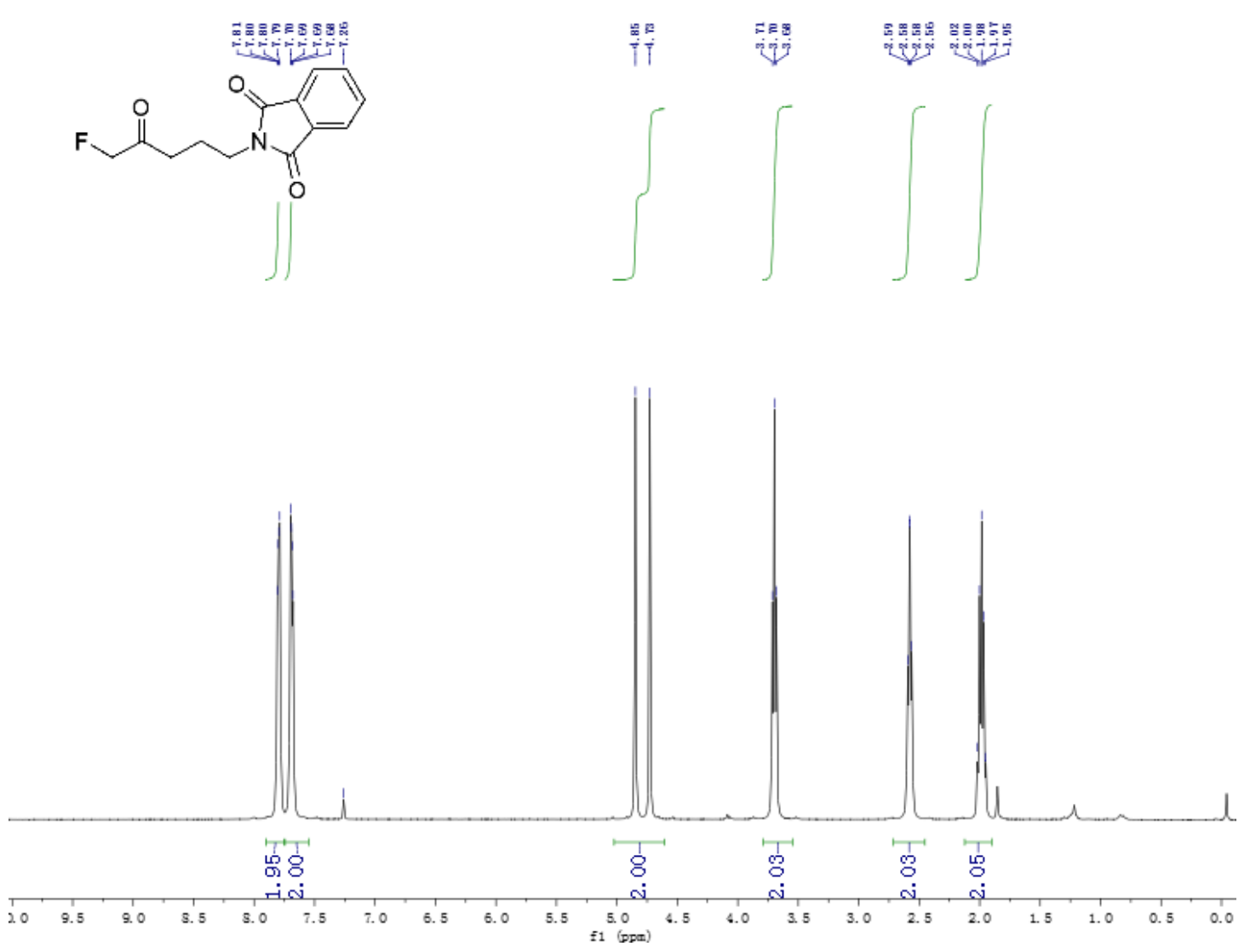

79 
${ }^{13} \mathrm{C}$ NMR of $2 \mathbf{x}$
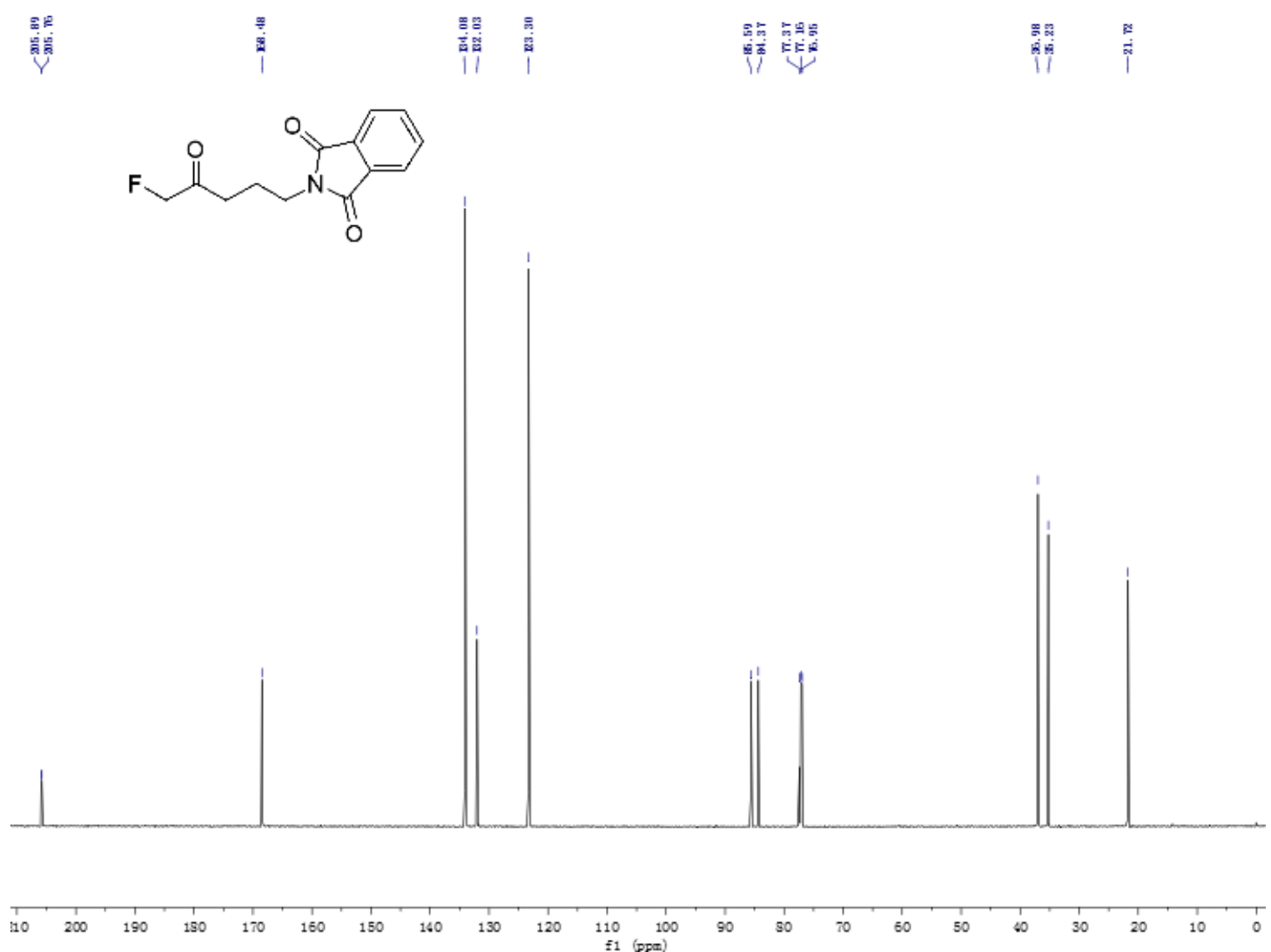

${ }^{19}$ F NMR of $2 \mathbf{x}$

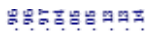

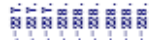<smiles>O=C(CF)CCCN1C(=O)c2ccccc2C1=O</smiles>

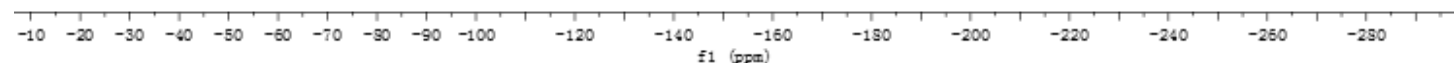


${ }^{1} \mathrm{H}$ NMR of $\mathbf{2 y}$
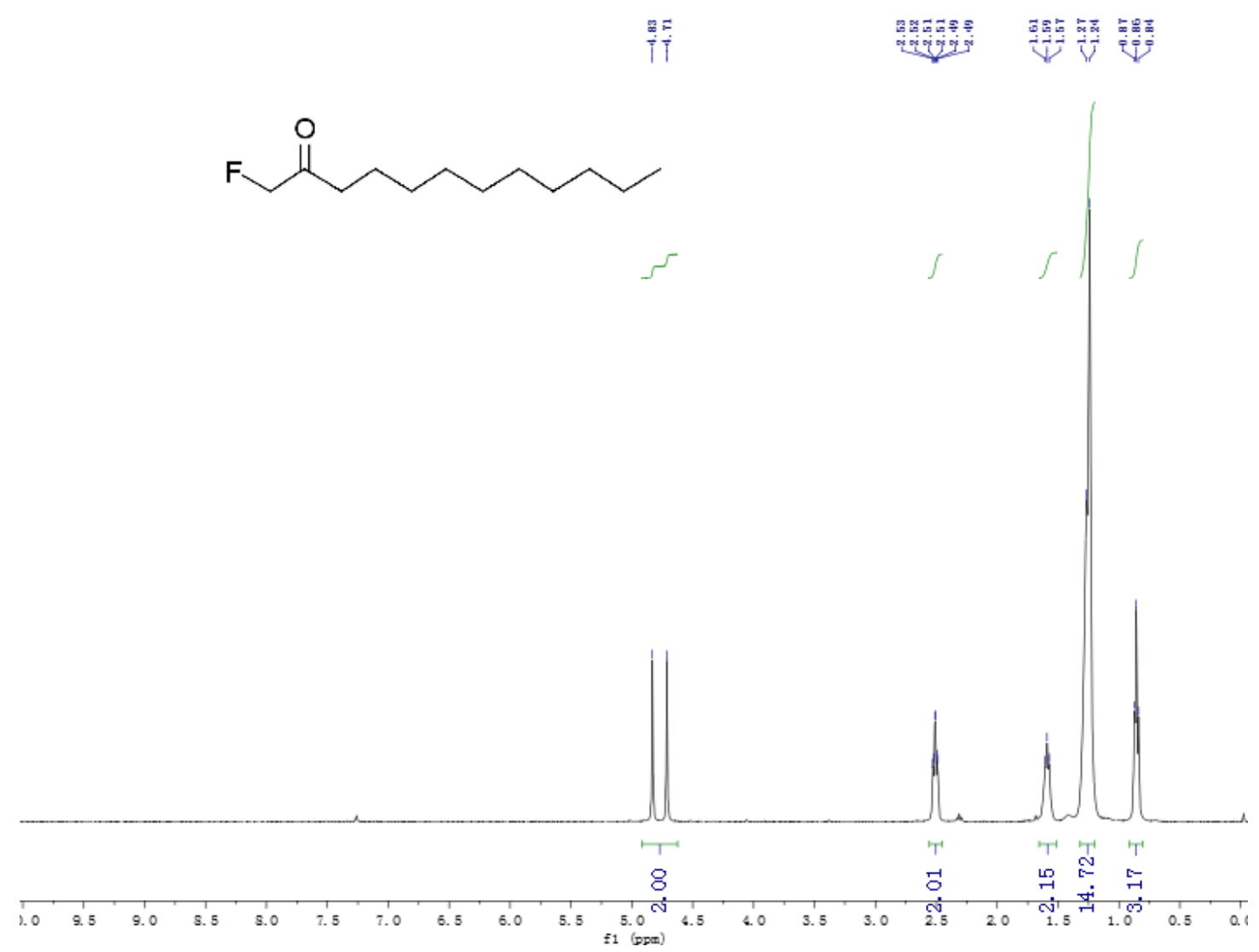

${ }^{13} \mathrm{C}$ NMR of $\mathbf{2 y}$

믐

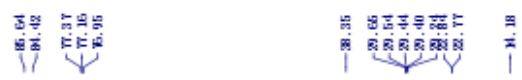

监

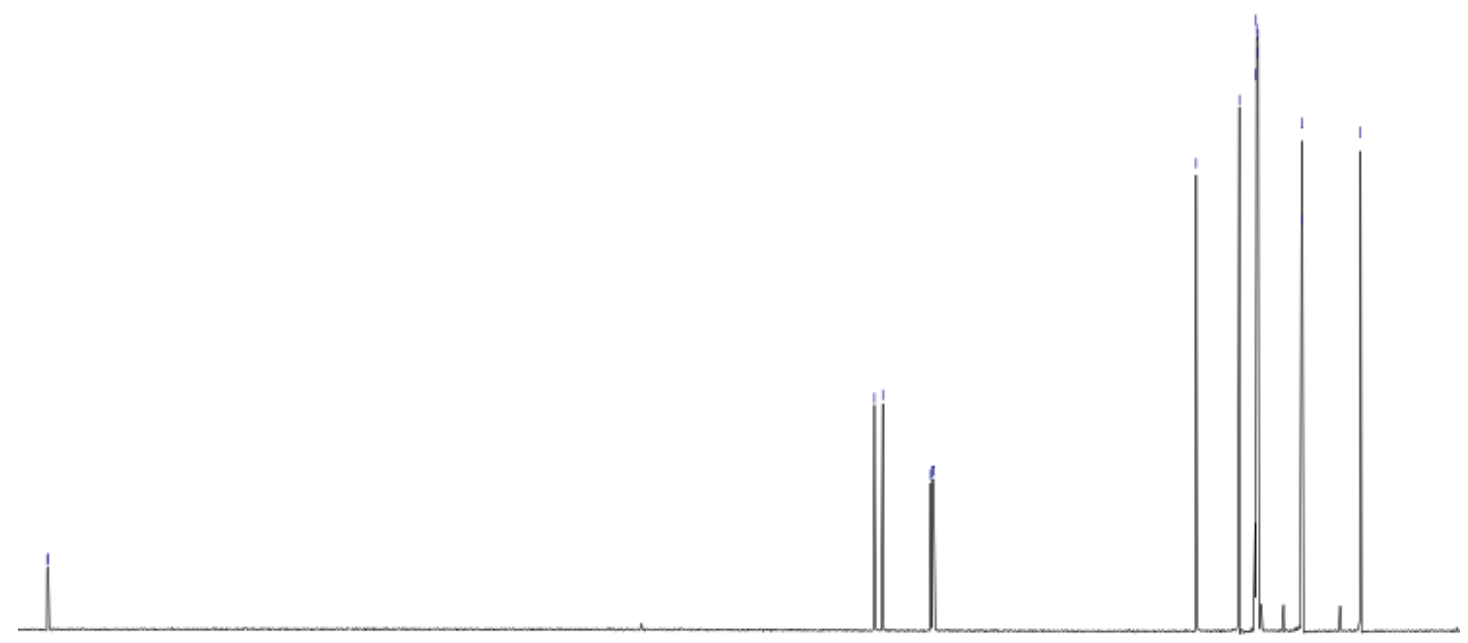

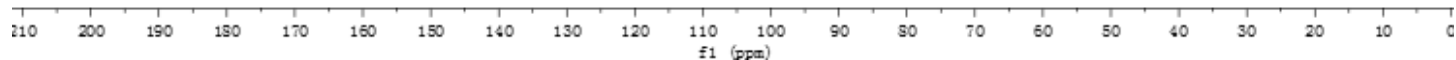


${ }^{19}$ F NMR of $\mathbf{2 y}$

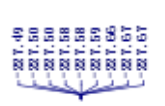

监 N

สูสสรส

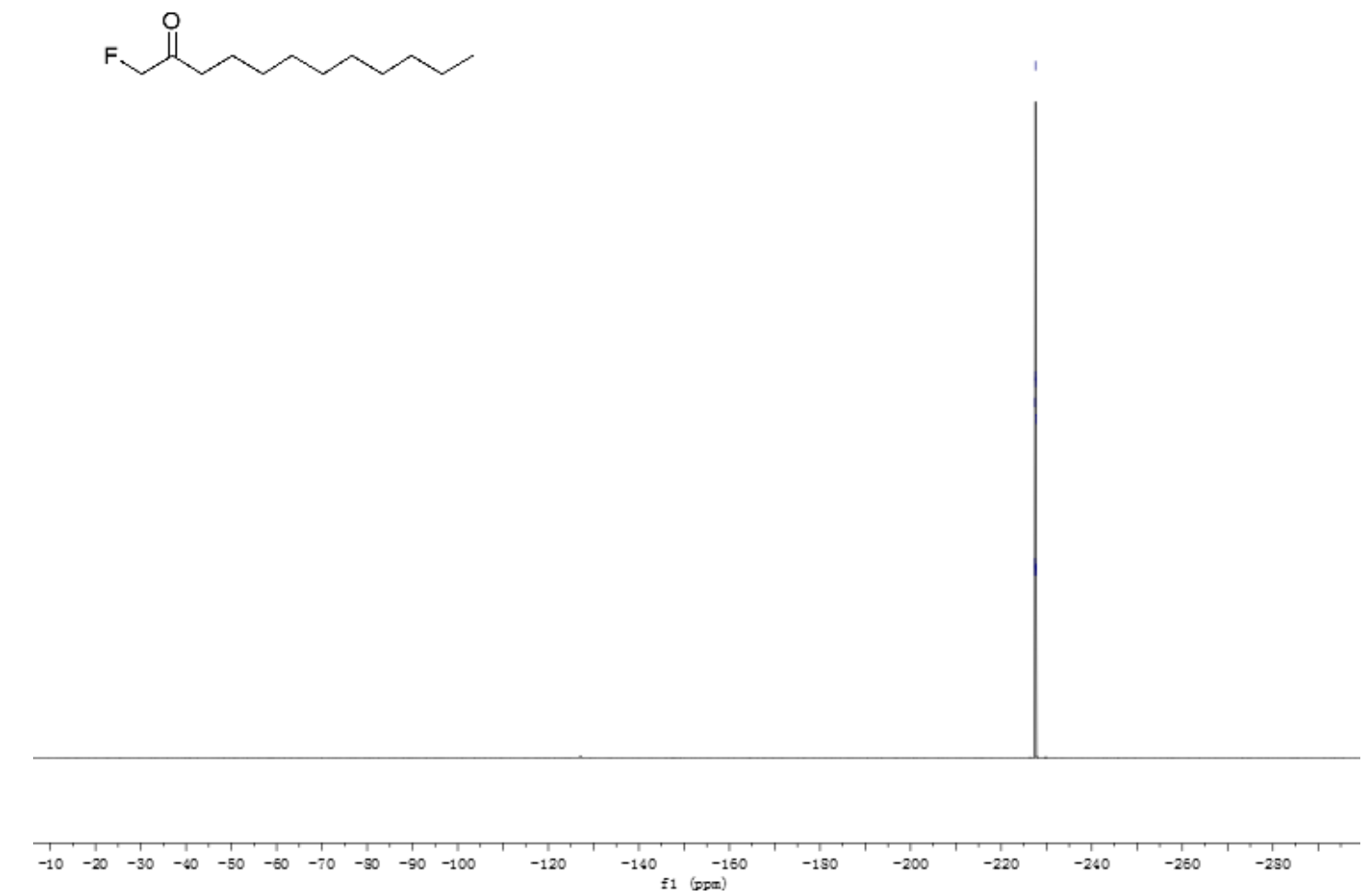

${ }^{1} \mathrm{H}$ NMR of $\mathbf{2 z}$

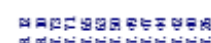

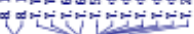

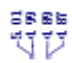

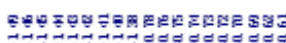

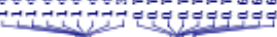<smiles>O=C(c1ccc(-c2ccccc2)cc1)C(F)C1CC1</smiles>
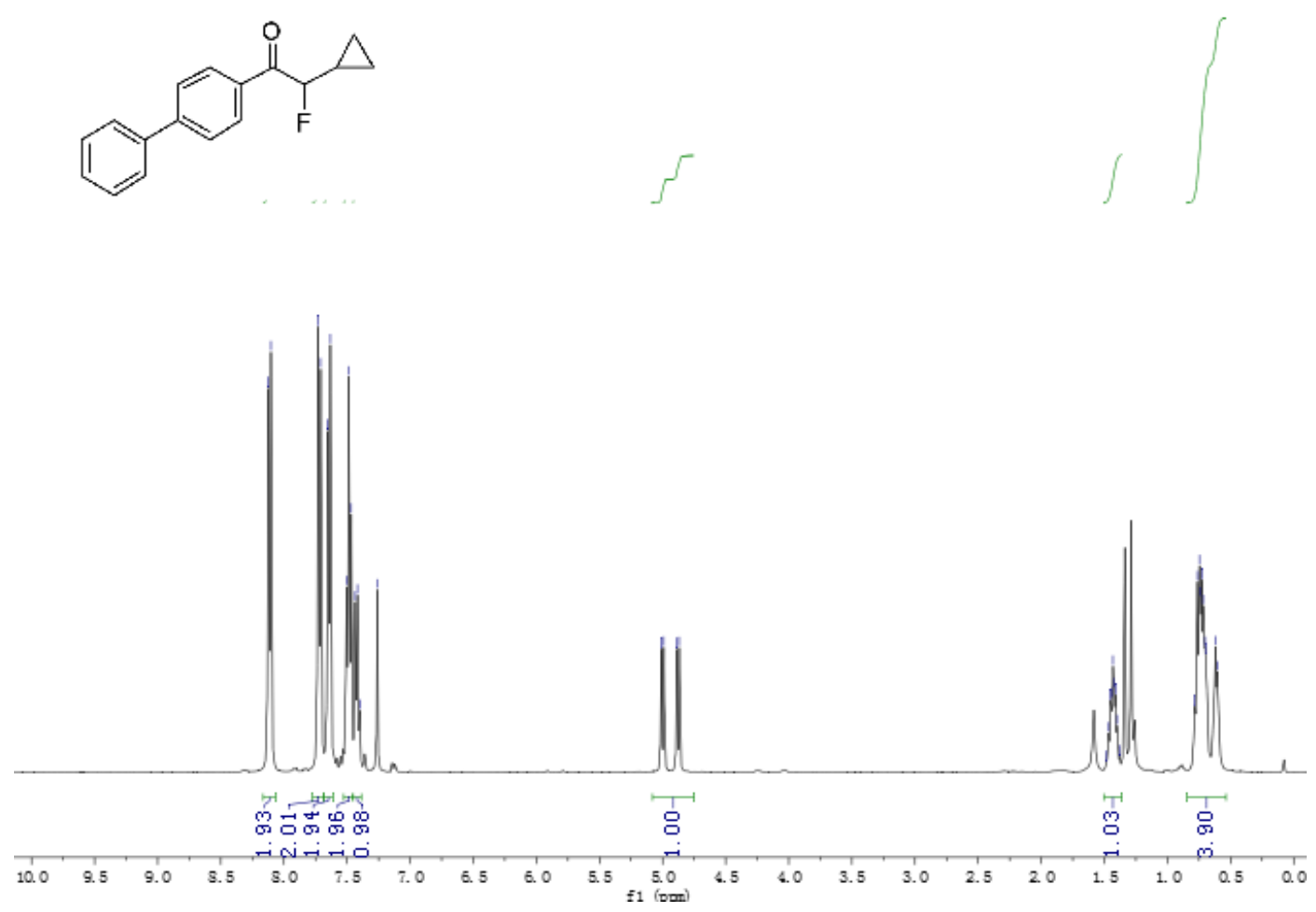
${ }^{13} \mathrm{C}$ NMR of $\mathbf{2 z}$

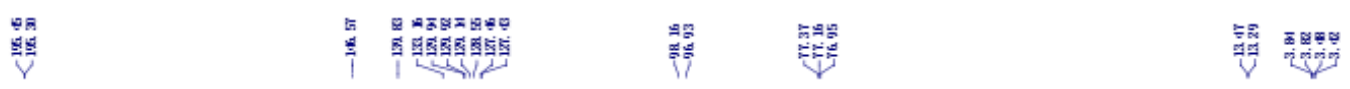<smiles>O=C(c1ccc(-c2ccccc2)cc1)C(F)C1CC1</smiles>
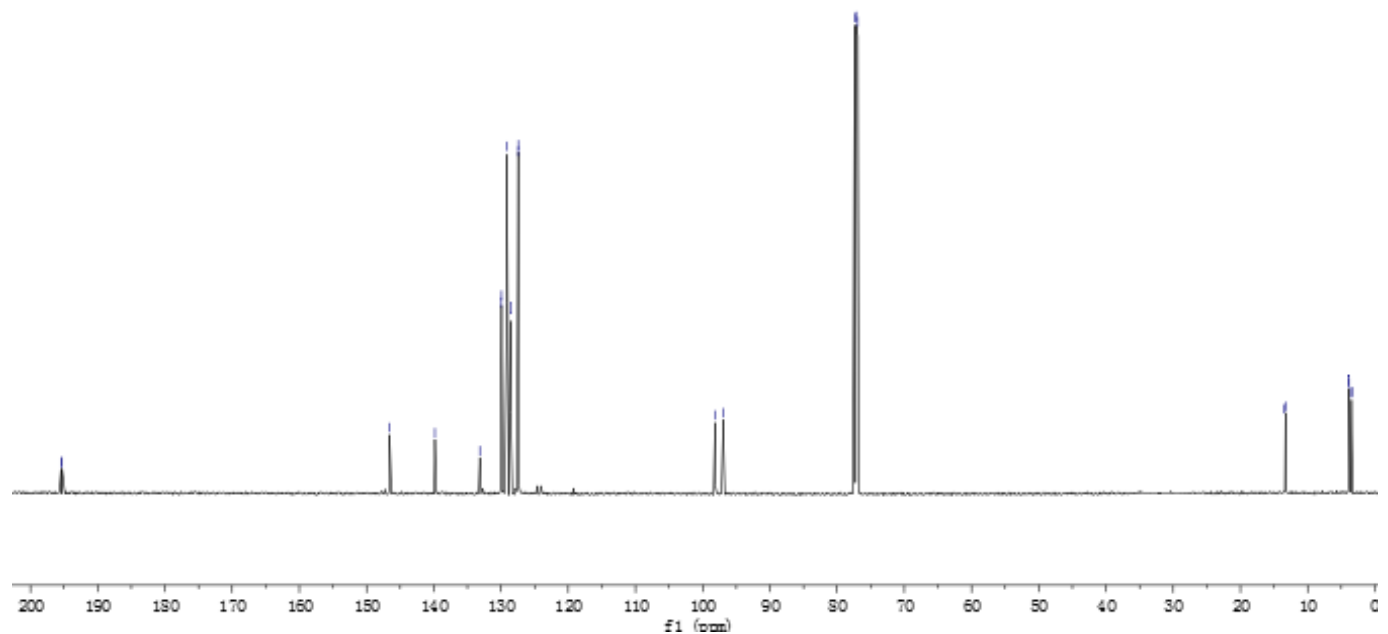

${ }^{19} \mathrm{~F}$ NMR of $\mathbf{2 z}$

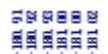<smiles>O=C(c1ccc(-c2ccccc2)cc1)C(F)C1CC1</smiles>

垈

$\begin{array}{llllllllll}-10 & -20 & -30 & -40 & -50 & -60 & -70 & -50 & -90 & -100\end{array}$

$-120 \quad-160 \quad-160$ 
${ }^{1} \mathrm{H}$ NMR of 3
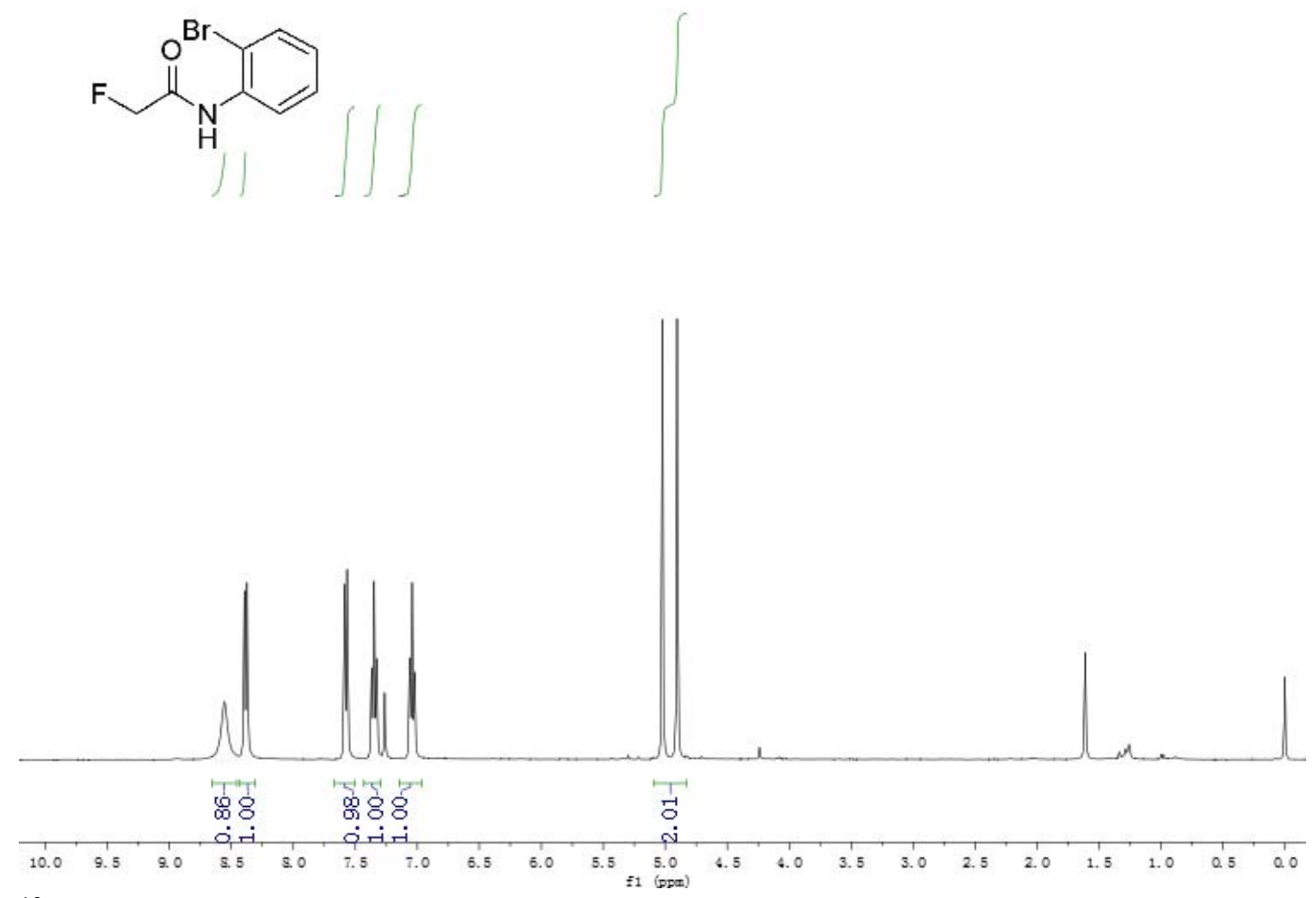

${ }^{13} \mathrm{C}$ NMR of 3
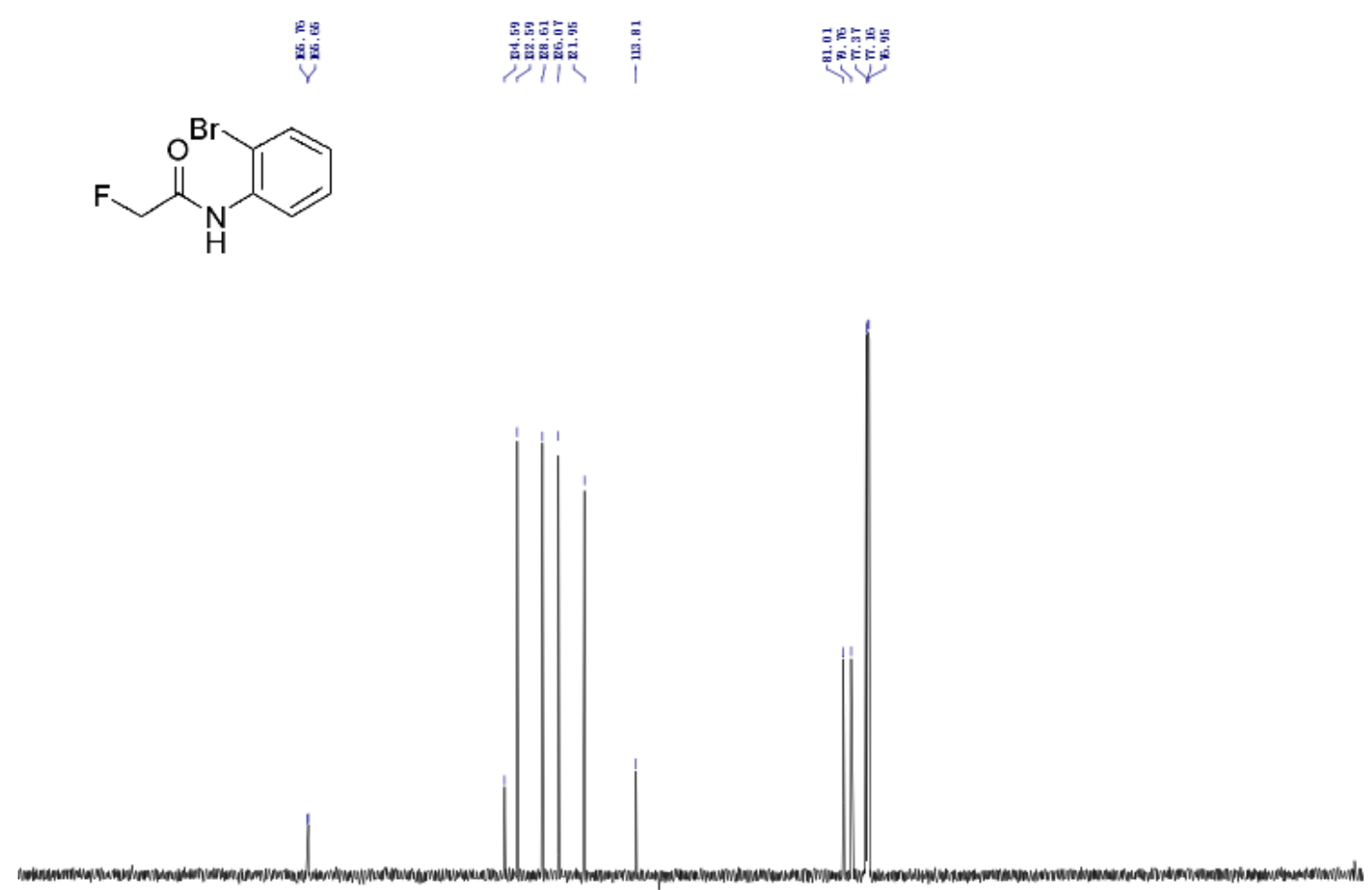

$\begin{array}{llllllllllllllllllllllllll}210 & 200 & 190 & 150 & 170 & 160 & 150 & 140 & 130 & 120 & 110 & 100 & 90 & 90 & 70 & 60 & 50 & 40 & 30 & 20 & 10 & 0\end{array}$ 
${ }^{19} \mathrm{~F}$ NMR of 3

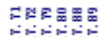

สำสสส

$\mathrm{N}_{\mathrm{H}}^{\mathrm{Br}}$

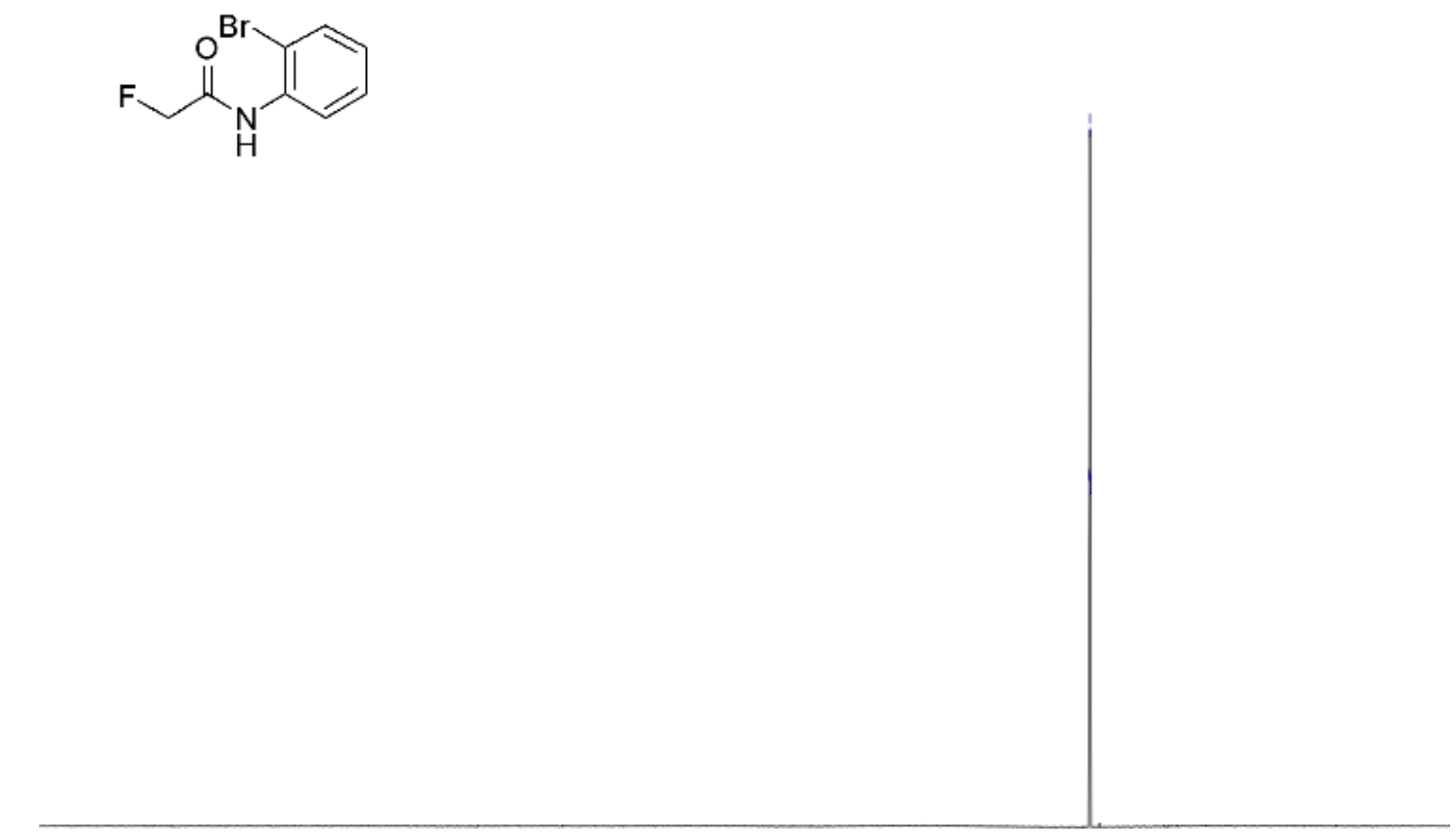

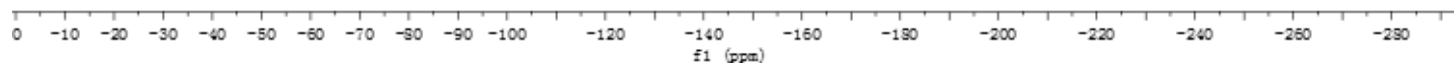

${ }^{1} \mathrm{H}$ NMR of $\mathbf{3 a}$

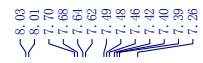<smiles>CC1(C)CCCC(C)(C)N1OCC(=O)c1ccc(-c2ccccc2)cc1</smiles>
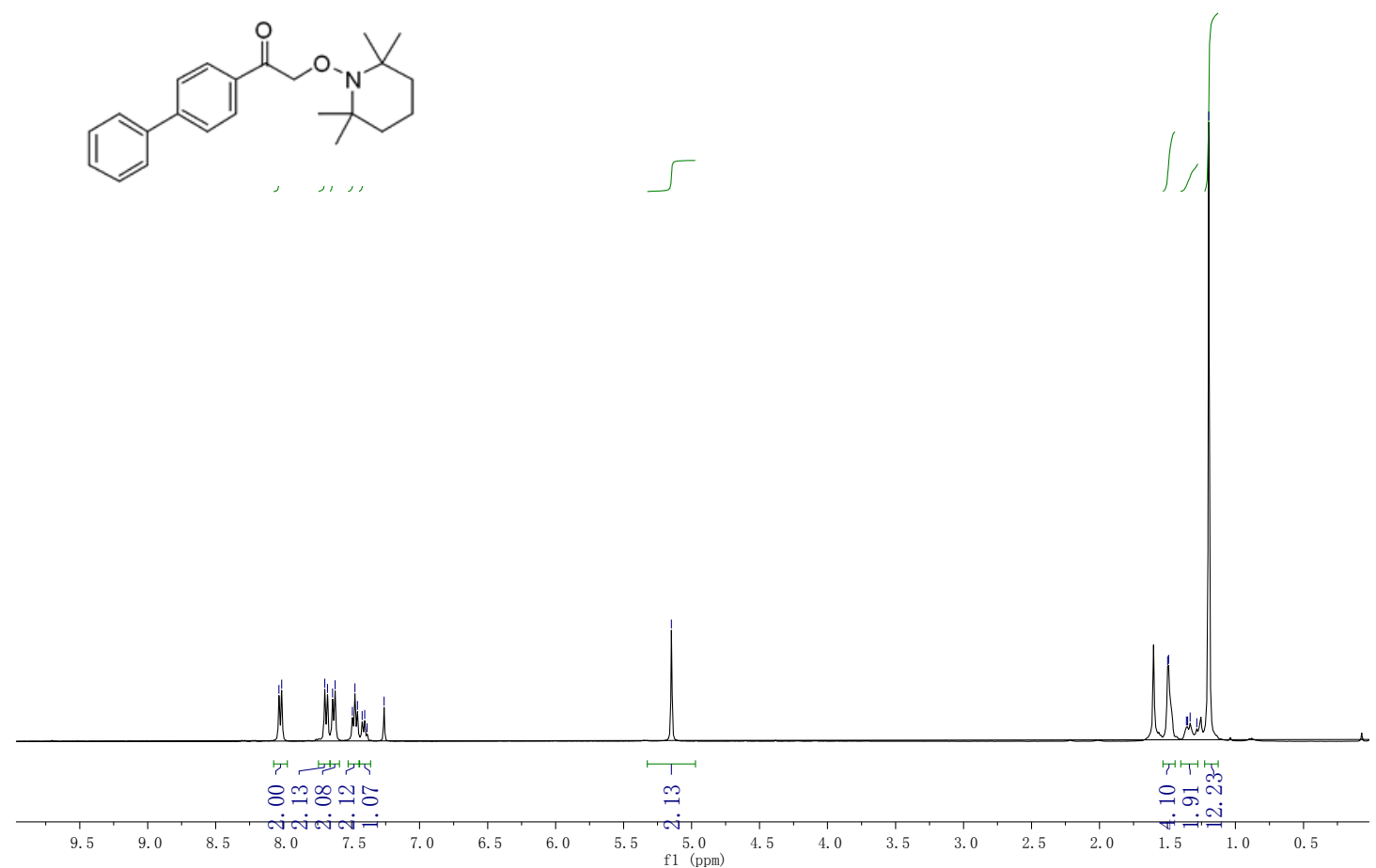
${ }^{13} \mathrm{C}$ NMR of $3 a$

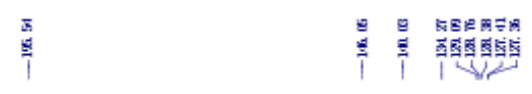

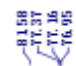
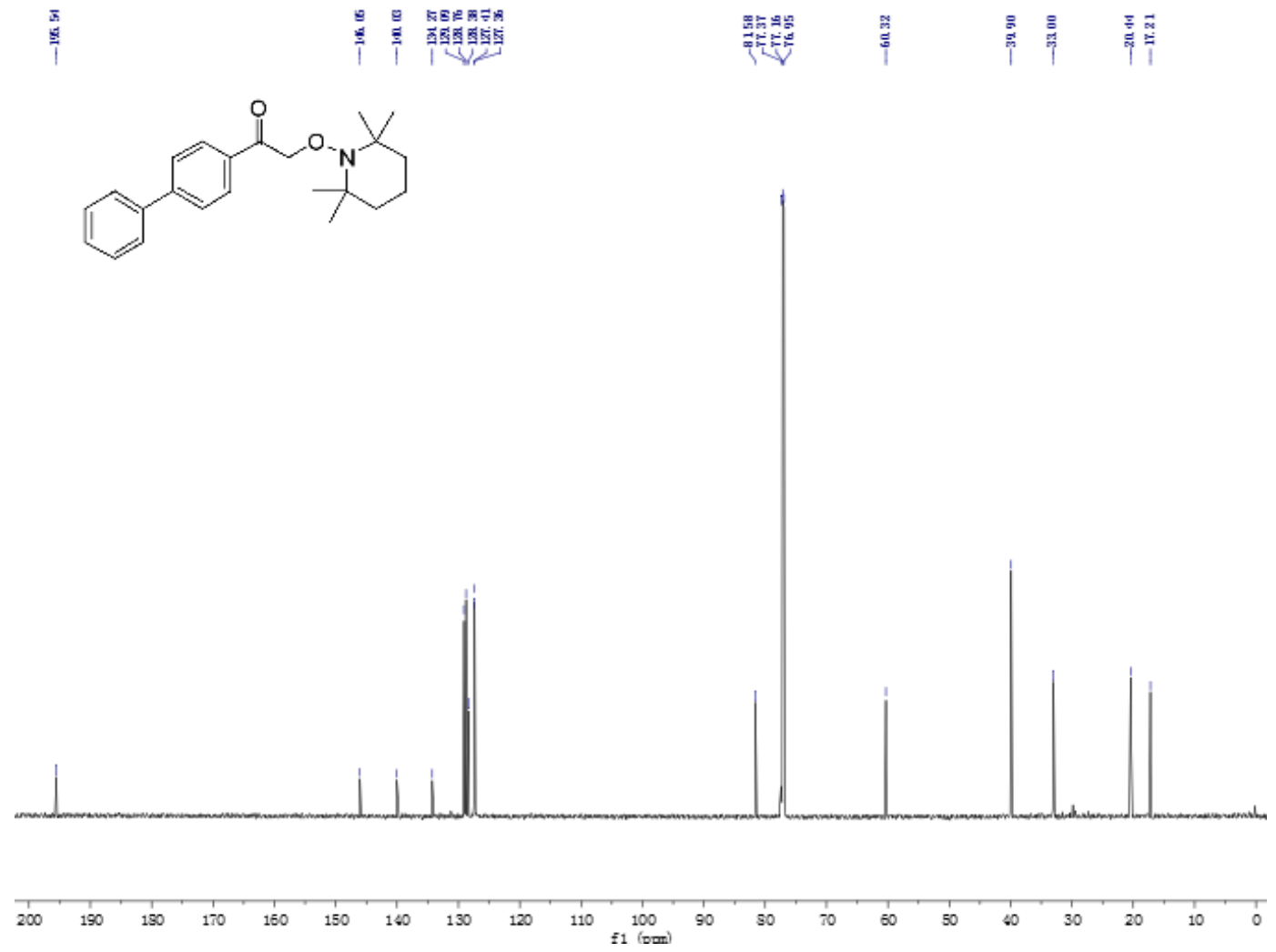

${ }^{1} \mathrm{H}$ NMR of $\mathbf{3 b}$
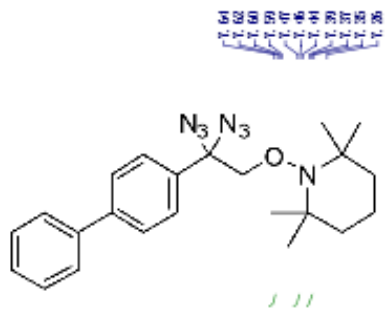

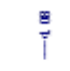

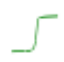

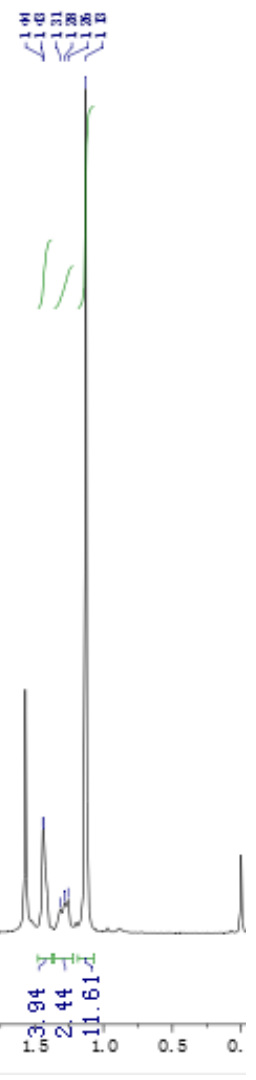

86 
${ }^{13} \mathrm{C}$ NMR of $\mathbf{3 b}$
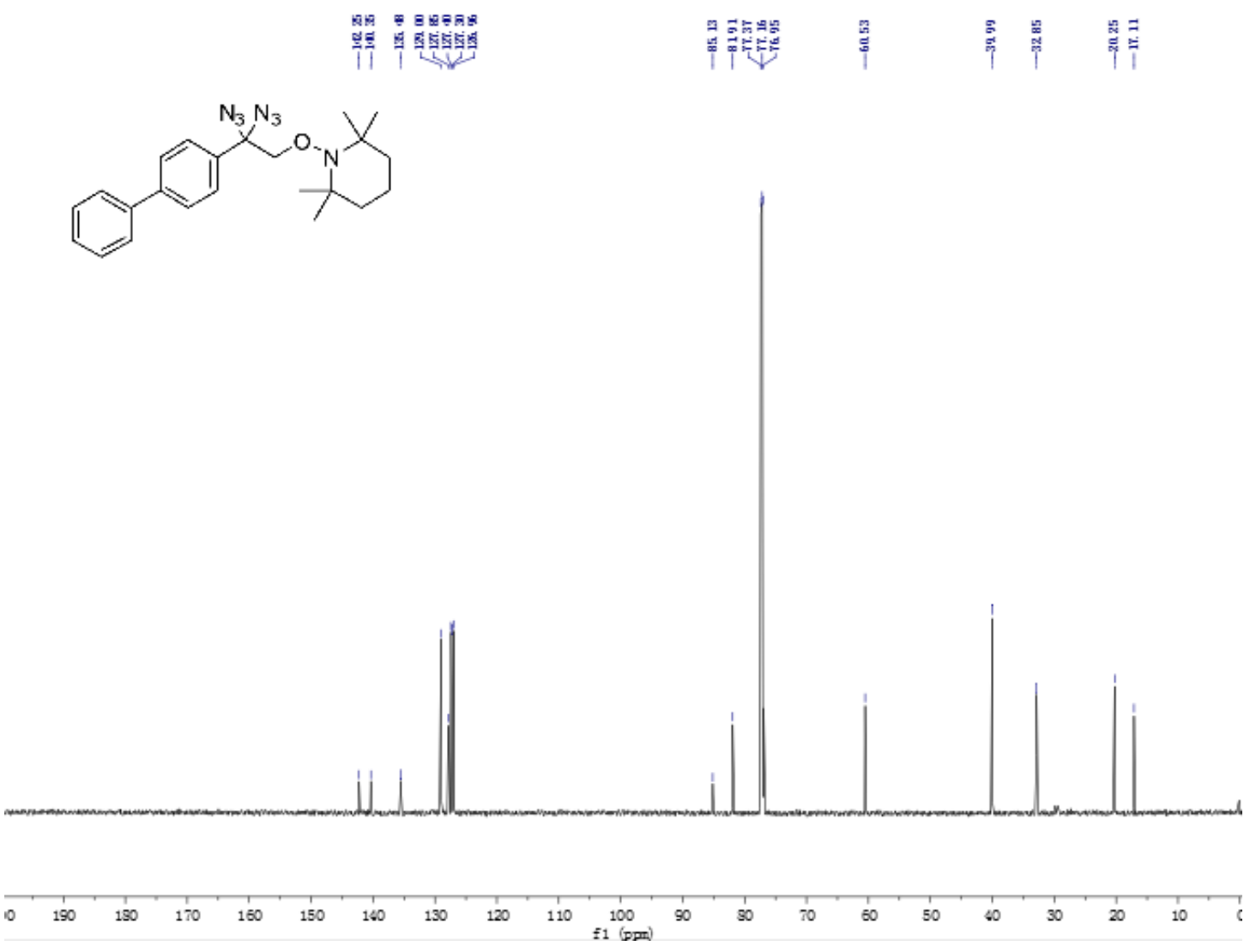

87 